UNIVERSIDADE DE SÃO PAULO

INSTITUTO DE FÍSICA

\title{
Espectro e dimensão Hausdorff de operadores bloco-Jacobi com perturbações esparsas distribuídas aleatoriamente
}

\section{Silas Luiz de Carvalho}

Orientador: Prof. Dr. Domingos H. U. Marchetti

Tese de Doutorado

submetida ao Instituto de Física

da Universidade de São Paulo

para a obtenção do título de

Doutor em Ciências.

Comissão Examinadora:

Prof. Dr. César Rogério de Oliveira (UFSCar)

Prof. Dr. Henrique von Dreifus (IME-USP)

Prof. Dr. João Carlos Alves Barata (IFUSP)

Prof. Dr. Paulo Teotônio Sobrinho (IFUSP)

Prof. Dr. Domingos Humberto Urbano Marchetti (IFUSP) 



\section{Agradecimentos}

A Isis, minha esposa e amiga, pelo carinho, pela dedicação e pelo incondicional apoio.

Aos meus pais Nilza Latorre Taranha e Sebastião de Carvalho, aos meus irmãos Silvia, Sergio, Selma e Solange, aos meus avós Edú e Lucrécia, e a minha tia Anameiry, tão importantes para mim.

Ao meu orientador Domingos Humberto Urbano Marchetti e meu ao co-orientador Walter Felipe Wreszinski, pela atenção, pelo cuidado e sobretudo pela dedicação.

A todos os meus amigos, que sempre me apoiaram nos momentos bons e difíceis.

Às secretárias e demais funcionários do Departamento de Física Geral, pela prestatividade e pela amizade.

À Fundação de Amparo à Pesquisa do Estado de São Paulo (FAPESP), pelo apoio financeiro. 



\section{Resumo}

Neste trabalho buscamos caracterizar o espectro de uma classe de operadores blocoJacobi limitados definidos em $l^{2}\left(\Lambda, \mathbb{C}^{L}\right)\left(\Lambda: \mathbb{Z}_{+} \times\{0,1, \ldots, L-1\}\right.$ representa uma faixa de largura $L \geq 2$ no semi-plano $\mathbb{Z}_{+}^{2}$ ) e sujeitos a perturbações esparsas (no sentido que as distâncias entre as "barreiras" crescem geometricamente à medida que estas se afastam da origem) distribuídas aleatoriamente. Tais operadores são construídos a partir da soma de Kronecker de matrizes de Jacobi $J$, cada qual atuando em uma direção do espaço. Demonstramos, por meio da bloco-diagonalização do operador, que suas principais propriedades espectrais dependem da caracterização da "medida de mistura" $\frac{1}{L} \sum_{j=0}^{L-1} \mu_{j}, \mu_{j}$ a medida espectral associada à matriz de Jacobi $J^{j}=J+2 \cos (2 \pi j / L) I$. Para tanto, buscamos primeiramente caracterizar cada uma das medidas $\mu_{j}$, explorando e aperfeiçoando algumas técnicas bastante conhecidas no estudo de operadores esparsos unidimensionais. Demonstramos, por exemplo, que a seqüência de ângulos de Prüfer (variáveis que, juntamente com os raios de Prüfer, parametrizam as soluções da equação de autovalores) é uniformemente distribuída no intervalo $[0, \pi)$, o que nos permite determinar o comportamento assintótico médio das soluções da equação de autovalores. Tal resultado, aliado às técnicas desenvolvidas por Marchetti et. al. em [MWGA] e a uma adaptação dos critérios de Last e Simon [LS1] para operadores esparsos, nos permitem demonstrar a existência de uma transição aguda (pontual) entre os espectros singular-contínuo e puramente pontual. Empregamos em seguida os resultados de Jitomirskaya e Last presentes em [JL] e obtemos a dimensão Hausdorff exata associada à medida $\mu_{j}$, dada por $\alpha_{j}=1+\frac{4\left(1-p^{2}\right)^{2}}{p^{2}\left(4-(\lambda-2 \cos (2 \pi j / L))^{2}\right)}(\lambda \in[-2,2])$, recuperando um resultado análogo obtido por Zlatoš em [Z]. Por fim, adaptamos tais resultados à situação da medida de mistura associada à matriz bloco-Jacobi, obtendo $\alpha=\min _{j \in \mathcal{I}(\lambda)} \alpha_{j}$, $\mathcal{I}(\lambda):\{m \in\{0,1, \ldots, L-1\}: \lambda \in[-2+2 \cos (2 \pi j / L), 2+2 \cos (2 \pi j / L)]\}$, como sua dimensão Hausdorff exata. Estudamos modelos idênticos com esparsidades sub e supergeométricas, obtendo na primeira situação um espectro puramente pontual (de dimensão Hausdorff nula) e na segunda um espectro puramente singular-contínuo (de dimensão Hausdorff 1). Finalmente, verificamos a existência de transição entre os espectros puramente pontual e singular-contínuo em um modelo com esparsidade super-geométrica cuja dimensão Hausdorff associada à medida espectral é nula. 



\section{Abstract}

In this work we attempt to caracterize the spectrum of a class of limited block-Jacobi operators defined in $l^{2}\left(\Lambda, \mathbb{C}^{L}\right)\left(\Lambda: \mathbb{Z}_{+} \times\{0,1, \ldots, L-1\}\right.$ represents a strip of width $L \geq 2$ on the semi-plane $\mathbb{Z}_{+}^{2}$ ) subject to a sparse perturbation (which means that the distance between the "barries" grow geometrically with their distance to the origin) randomly distributed. Such operators are defined as Kronecker sums of unidimensional Jacobi matrices $J$, each one acting in different directions of the space. We prove, by means of a block-diagonalization of the operator, that its most relevant spectral properties depend on the caracterization of the "mixture measure" $\frac{1}{L} \sum_{j=0}^{L-1} \mu_{j}, \mu_{j}$ the spectral measure of the Jacobi matrix $J^{j}=J+2 \cos (2 \pi j / L) I$. For this, we must characterize at first each one of the measures $\mu_{j}$, exploiting and improving some well known techniques developed in the study of unidimensional sparse operators. We prove, for instance, that the sequence of Prüfer angles (variables which parametrize the solutions of the eigenvalue equation) are uniform distributed on the interval $[0, \pi)$, a result which gives us condition to determine the average asymptotic behavior of the solutions of the eigenvalue equation. Such result, in association with the techniques developed by Marchetti et. al. in [MWGA] and with an adaptation of Last-Simon [LS1] criteria for sparse operator, permit us to prove the existence of a sharp transition between singular continuous and pure point spectra. Following on, we use the results from Jitomirskaya-Last of [JL] and obtain the exact Hausdorff dimension of the measure $\mu_{j}$, given by $\alpha_{j}=1+\frac{4\left(1-p^{2}\right)^{2}}{p^{2}\left(4-(\lambda-2 \cos (2 \pi j / L))^{2}\right)}$ $(\lambda \in[-2,2])$, recovering an analogous result due to Zlatoš in [Z]. At last, we adapt these results to the mixture measure of the block-Jacobi matrix, obtaining $\alpha=\min _{j \in \mathcal{I}(\lambda)} \alpha_{j}$, $\mathcal{I}(\lambda):\{m \in\{0,1, \ldots, L-1\}: \lambda \in[-2+2 \cos (2 \pi j / L), 2+2 \cos (2 \pi j / L)]\}$, as its exact Hausdorff dimension. We study as well identical models with sub and super geometric sparsities conditions, obtaining a pure point spectrum (with null Hausdorff dimension) in the first case, and a purely singular continuous spectrum (such that its Hausdorff dimension is 1) in the second. Finally, we prove the existence of a transition between pure point and singular continuous spectra in a model with sub-geometric sparsity whose Hausdorff dimension related to the spectral measure is null. 



\section{Sumário}

1 Introdução $\quad 11$

1.1 Motivações e contextualização do trabalho . . . . . . . . . . . . . . . . 11

1.2 Modelo estudado . . . . . . . . . . . . . . . . . . . . . . . 20

1.3 Roteiro de leitura . . . . . . . . . . . . . . . . . . . . . . . . 29

2 Medida espectral e espectro essencial do operador bloco-Jacobi $\mathcal{J}_{\delta, \phi} \quad 31$

2.1 Bloco-diagonalização das matrizes $\mathcal{J}_{\delta, \phi} \ldots \ldots \ldots$. . . . . . . . 31

2.2 Funções de Green e $M$ de Weyl Titchmarsh matriciais e a medida espectral 32

2.3 Caracterização da medida espectral matricial $\Omega \ldots \ldots$. . . . . . . . . . . . . . . . . .

2.4 Estudo do espectro de $\mathcal{J}_{\delta, \phi} \ldots \ldots \ldots \ldots \ldots$. . . . . . . . . . . 41

2.4.1 Características espectrais da matriz livre $\mathcal{J}_{0} \ldots \ldots$. . . . . . . . . . . . . 41

2.4 .2 Espectro essencial da matriz $\mathcal{J}_{\delta, \phi} \ldots \ldots \ldots$. . . . . . . . . 43

3 Existência de transição espectral $\quad 47$

3.1 Matrizes de transferência e variáveis de Prüfer adaptadas . . . . . . . . . . 49

3.1.1 Determinação do comportamento assintótico da seqüência $\left(R_{n}^{j}\left(\theta_{0}^{j}\right)\right)_{n \geq 0} 51$

3.2 Distribuição uniforme dos ângulos de Prüfer . . . . . . . . . . . . . . . 58

3.3 Demonstração da existência de transição espectral . . . . . . . . . . . . . . 63

4 Dimensão Hausdorff da medida de mistura $\quad \mathbf{7 1}$

4.1 Medidas espectrais $\mu_{j} \ldots \ldots \ldots \ldots \ldots \ldots$. . . . . . . . . . . . . . . . . . . . . . .

4.2 Medida espectral $\Omega_{00} \ldots \ldots \ldots \ldots \ldots \ldots$

5 Modelos com esparsidades sub-geométrica e super-geométrica $\quad 81$

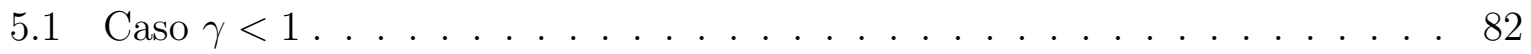

5.2 Casos em que $\gamma>1 \ldots \ldots \ldots \ldots$

6 Conclusões e problemas em aberto $\quad 89$

Apêndices: $\quad 95$

A Propriedades espectrais de operadores limitados $\quad 95$

A.1 Espectro . . . . . . . . . . . . . . . . . . . . . 97 
B Teorema espectral

B.1 Teorema espectral multiplicativo e a decomposição das medidas espectrais 105

B.2 Projetores espectrais . . . . . . . . . . . . . . . . 111

C Funções Herglotz

D Função $m$ de Weyl-Titchmarsh e seu papel na teoria espectral

D.1 Definições de matriz de Jacobi e função $m$. . . . . . . . . . . . . . . . . 121

D.2 Ligação entre a função $m$ e a função de Green . . . . . . . . . . . . . . 126

D.3 Medida espectral e sua relação com comportamento de fronteira da função de Green . . . . . . . . . . . . . . . . . . . . . 129

D.4 Medida espectral e comportamento de fronteira da função $m$. . . . . . . . 133

E Papel da subordinância na teoria espectral

F Ferramentas utilizadas na determinação da natureza espectral

F.1 Definição da matriz de transferência . . . . . . . . . . . . . . . . . . . . . . 145

F.2 Critérios de Last e Simon . . . . . . . . . . . . . . . . . . . . 146

F.3 Variáveis de Prüfer . . . . . . . . . . . . . . . . . . . 156

G Medidas e dimensão Hausdorff 161

H Caracterização do espectro singular: teoria de Jitomirskaya e Last 167

$\begin{array}{lr}\text { Referências Bibliográficas } & 177\end{array}$ 


\section{Capítulo 1}

\section{Introdução}

\subsection{Motivações e contextualização do trabalho}

Um dos modelos mais estudados por físicos e matemáticos na segunda metade do século passado e no século presente é o modelo de Anderson "tight-binding", apresentado pela primeira vez em $[\mathrm{A}]$, que descreve elétrons não-interagentes em um potencial aleatório. Sabemos que o movimento dos elétrons em um metal ou cristal perfeitos (onde os átomos formam estruturas perfeitamente periódicas, livres de impurezas) é descrito pela teoria de Bloch, adequada apenas à descrição de algumas poucas propriedades de equilíbrio dos sólidos: esta deve ser substituída por uma teoria cinética na qual os elétrons interajam com as várias impurezas presentes no material. O modelo de Anderson é uma das primeiras e mais bem sucedidas destas teorias, em que a aleatoriedade do potencial representa as impurezas presentes no material e é responsável pela transição entre os possíveis estados eletrônicos.

O modelo "tight-binding", originalmente proposto em termos dos operadores de criação e aniquilação de elétrons $a_{i}^{\dagger}, a_{i}$, para cada sítio $i$, tem como hamiltoniano

$$
H=\sum_{i} \epsilon_{i} a_{i}^{\dagger} a_{i}+\sum_{i, j} V_{i j} a_{i}^{\dagger} a_{i}
$$

em que $V_{i j}=-V$ para sítios primeiros vizinhos e $V_{i j}=0$ de outra forma, e as variáveis aleatórias $\epsilon_{i}$ são tomadas como independentes e uniformemente distribuídas entre $-W / 2$ e $W / 2$.

Analisemos, no entanto, um hamiltoniano um pouco diferente do definido acima e muito mais próximo do que vamos tratar em nosso trabalho. Seja $\mathcal{H}$ o espaço de Hilbert das funções complexas quadrado-somáveis em $\mathbb{Z}^{\nu}, \nu \geq 1$. Vale destacar que o uso do modelo discreto é perfeitamente justificável do ponto de visto físico, já que o modelo "tight-binding" é, em certo sentido, uma aproximação do fenômeno real; os elétrons, nesse caso, "saltam" um a um entre sítios sucessivos (que representam átomos, em boa aproximação fixos (baixa temperatura), presentes na rede cristalina). 
O operador de Schrödinger definido em $\mathcal{H}$ é

$$
H=-\Delta+V
$$

em que $\Delta$ é o laplaciano de diferença finita (sem o termo diagonal $-2 \nu$ ) e $V$ é um potencial aleatório. Repare que $\Delta$ é um operador limitado, cujos elementos de matriz satisfazem $\Delta_{i j}=-1$ se $|i-j|=1$ e $\Delta_{i j}=0$ caso contrário. No espaço de Fourier, $\Delta$ corresponde ao operador de multiplicação por $2 \sum_{i=1}^{\nu} \cos k_{i},-\pi<k_{i} \leq \pi$, de modo que $\Delta$ apresenta espectro absolutamente contínuo (Definição B.1.14) no intervalo $[-2 \nu, 2 \nu]$. O potencial $V$ é geralmente definido como uma família de variáveis aleatórias independentes e identicamente distribuídas (uniformemente distribuídas no modelo original de Anderson), $V_{j}$, $j \in \mathbb{Z}^{\nu}$, agindo como operadores de multiplicação em $\mathcal{H}$.

Um dos objetos de estudo mais interessantes, relacionado ao modelo de Anderson e a sistemas desordenados em geral, é a densidade de estados. Além de sua óbvia importância física, já que pode ser medida em alguns experimentos, a obtenção da densidade de estados é um problema desafiador em matemática. Um dos principais objetos analisados neste trabalho é a natureza espectral de alguns operadores de Schrödinger, que depende diretamente da densidade de estados (vide a Seção 4 de $[\mathrm{K}]$ ). Apesar do apelo físico (já que esta é em um certo sentido uma média do número de níveis de energia por célula da rede; no caso unidimensional, uma célula equivale a um segmento unitário), a denominação "densidade de estados" é matematicamente incorreta. De fato, o objeto que analisamos é a densidade espectral (os detalhes se encontram no Apêndice D), que como veremos não apresenta necessariamente uma densidade no sentido matemático usual.

$\mathrm{Na}$ teoria dos metais em estado sólido, sempre se acreditou que um espalhamento fraco, ligado a uma desordem fraca, mudaria os autovetores (em um sentido generalizado quando tratamos de sistemas infinitos) das ondas de Bloch para uma superposição de estados que apresentam, grosseiramente, a mesma energia. Esta superposição supostamente produziria autovetores generalizados estendidos (associados à região absolutamente contínua do espectro, onde de fato existe uma verdadeira densidade de estados), similares às ondas planas de Bloch. Dessa forma, quando Anderson (vide [A]) mostrou que uma desordem suficientemente grande poderia acarretar numa localização de todos os autovetores generalizados em alguma região finita do espaço, com um decaimento exponencial fora dessa região, houve certa surpresa na comunidade interessada. Este resultado foi rigorosamente demonstrado por Gold'sheid, Molchanov e Pastur [GMP] e subseqüentemente por Kunz e Souillard [KS1]. Logo em seguida ao trabalho de Anderson, Mott e Twose [MT], e Landauer [Lan] demonstraram que qualquer desordem em um sistema unidimensional pode ser suficiente para causar uma localização exponencial de todos os autovetores generalizados.

\section{Localização}

Outra questão indubitavelmente relevante e diretamente associada à natureza da medida espectral (ou mais explicitamente, à sua densidade) é a da evolução temporal do 
pacote de onda $\psi_{t}=e^{-i t H} \psi_{0}, \psi_{0} \in \mathcal{H}$. Um dos problemas matemáticos mais desafiadores é justamente a obtenção, com grande precisão, do comportamento assintótico $(t \rightarrow \infty)$ de $\psi_{t}$.

Consideremos o alargamento de $\psi_{t}$ como medido por

$$
r^{2}(t)=\sum_{x}\left|\psi_{t}(x)\right|^{2} x^{2}=\left\|x e^{-i t H} \psi_{0}\right\|^{2}
$$

É possível mostrar (vide a Seção 1 de $[\mathrm{Sp}]$ ) que $r^{2}(t) \leq$ const $\cdot t^{2}$ caso $x \psi_{0}$ seja quadrado-somável. Se o potencial $V$ for periódico ou nulo, então $r^{2}(t)=\operatorname{const} \cdot t^{2}$, o que corresponde fisicamente a uma partícula se movendo a velocidade constante. No caso em que $V_{j}$ são variáveis aleatórias independentes e a desordem (representando o valor máximo assumido por estas variáveis) é grande, Anderson afirmou que $\lim \sup _{t} r^{2}(t) \leq$ const (para todo $\psi_{0}$ de suporte compacto), ou seja, existe localização do pacote de onda, já que este não se alarga. Isso corresponde à existência de uma fase isolante em um metal (não há transporte). Como discutido anteriormente, a ocorrência de localização em uma dimensão é um resultado bem conhecido. Em duas dimensões, espera-se também que haja localização para qualquer desordem [OW, AALR]; entretanto, não existe uma demonstração rigorosa dessa conjectura.

A desigualdade $r^{2}(t) \leq$ const implica na existência de espectro puramente pontual (vide a Seção B.1 para uma discussão do conceito) para $H$. Este fato segue do Teorema RAGE (vide, por exemplo, [RS4]), que afirma que o projetor espectral $P_{c}$ (Definição B.2.1) sobre o espectro contínuo de $H$ é

$$
\begin{aligned}
\left\|P_{c} \psi_{t}\right\|^{2} & =\lim _{R \rightarrow \infty} \lim _{T \rightarrow \infty} \frac{1}{T} \int_{0}^{T} \sum_{x}\left|\psi_{t}(x)\right|^{2} \chi_{x \geq R}(x) d t \\
& \leq \lim _{R \rightarrow \infty} \lim _{T \rightarrow \infty} \frac{1}{R^{2} T} \int_{0}^{T} \sum_{x}\left|\psi_{t}(x)\right|^{2} x^{2} d t \leq \lim _{R \rightarrow \infty} \frac{\text { const }}{R^{2}}=0
\end{aligned}
$$

onde $\chi_{A}(x)=1$ se $x \in A$ e $\chi_{A}(x)=0$ caso contrário é a chamada função característica do conjunto $A$.

Na situação em que $\nu>1$ e a desordem é grande, Martinelli e Scoppola [MS] demonstraram a ausência de espectro absolutamente contínuo demonstrando, com probabilidade 1, que para uma dada energia $E$ não existem soluções polinomialmente limitadas da equação de Schrödinger $(-\Delta+V-E) \psi=0$ (os detalhes se encontram na Seção 5.5 de [Sp]). Fröhlich, Martinelli, Scoppola e Spencer [FMSS] demonstraram a existência de localização e que o espectro é puramente pontual, com os autovetores generalizados decaindo exponencialmente rápido (vide as Seções 6-8 de [Sp] para um esboço da demonstração).

Para uma desordem fraca e $\nu>2$, espera-se que o espectro de $H$ contenha um intervalo $\left[E_{m}, E_{m}^{\prime}\right]$ puramente absolutamente contínuo; a tal intervalo devem corresponder os estados estendidos, como já discutido. As constantes $E_{m}$ e $E_{m}^{\prime}$ são chamadas de fronteiras de mobilidade, já que o pacote de onda associado ao elétron deve se alargar nesse intervalo 
de energias. Acredita-se que o alargamento do pacote de onda seja difusivo, isto é, que $r^{2}\left(t, \psi_{0}\right)=D t, D>0$, desde que $\psi_{0}$ não seja ortogonal aos estados estendidos. Existe condutividade caso a energia de Fermi do sistema (definida como sendo a energia do estado fundamental de um conjunto de elétrons) pertença à banda de mobilidade. Fora da banda, espera-se que o espectro seja puramente pontual, com os autovetores generalizados decaindo exponecialmente rápido. Kunz e Souillard [KS2] estabeleceram rigorosamente a existência de uma banda de mobilidade na rede de Bethe.

Sabe-se que mesmo para desordem fraca existem intervalos de espectro puramente pontual com funções exponencialmente localizadas (vide [FMSS]). Estes intervalos se encontram nas extremidades do espectro de $H$.

Mostremos a seguir como a localização exponencial do pacote de onda acarreta em uma condutividade estática média nula. Esta última é definida pela fórmula de Kubo,

$$
\bar{\sigma}(E)=\lim _{\varepsilon \rightarrow 0} \varepsilon^{2} \int \sum_{x}|G(0, x ; E+i \varepsilon)|^{2} x^{2} d P(V),
$$

em que $d P(V)$ representa a distribuição de probabilidade associada ao potencial $V$. A função de Green

$$
G(x, y ; E+i \varepsilon)=\left\langle(H-E-i \varepsilon)^{-1} \psi_{x}, \psi_{y}\right\rangle
$$

é, como definida acima, o núcleo integral ${ }^{\dagger}$ do resolvente $(H-z)^{-1}$ do operador $H$, para $z=E+i \varepsilon, \psi_{x, y}$ elementos de uma base ortonormal de $\mathcal{H}$ (a definição de resolvente se encontra no Apêndice A). Sabemos (vide a Seção D.3) que a função de Green apresenta uma ligação direta com a medida espectral.

Fröhlich e Spencer demonstraram em [FS] que para uma desordem e para uma energia suficientemente grandes, a função de Green satisfaz a desigualdade

$$
|G(0, x ; E+i \varepsilon)| \leq e^{m(K-|x|)},
$$

que segue para todo $x$, todo $K \geq 1$ e para $\varepsilon \neq 0$, com probabilidade no mínimo $1-C_{P} K^{-p}$, $p \geq 1, m$ e $C_{p}$ constantes positivas (Teorema 1.1 de [Sp]). Tomando $K=R+|x| / 2$ e a desigualdade $\varepsilon|G(x, y ; E+i \varepsilon)| \leq 1$ temos

$$
\varepsilon^{2} \int|G(0, x ; E+i \varepsilon)|^{2} x^{2} d P(V) \leq \varepsilon^{2} e^{2 m(R-|x| / 2)} x^{2}+\frac{C_{p} x^{2}}{(R+|x| / 2)^{p}}
$$

que quando somado em $x$ vai a zero nos limites $\varepsilon \rightarrow 0$ e $R \rightarrow \infty$. Demonstra-se, portanto, que a condutividade estática é nula na situação de localização exponencial. A constante de difusão $D(E)$ é proporcional a $\bar{\sigma}(E)$, e assim também se anula.

Uma contribuição fundamental para a questão da localização foi a de Simon e Wolff em [SW]. Ambos estenderam a teoria de Aronszajn [Ar] e Donoghue [Do], que trata da instabilidade da parte singular do espectro sob perturbações de posto um (vide a Seção

\footnotetext{
†Trata-se, na realidade, de um núcleo somável; os detalhes se encontram na Seção D.3.
} 
D.4 para uma discussão), para o problema de potenciais aleatórios, incluindo o modelo de Anderson multidimensional.

Seja $H$ o hamiltoniano (1.1) associado ao modelo de Anderson e defina $A=H-$ $V(0) P, P=\left(\delta_{0}, \dot{)} \delta_{0}\right.$ a projeção sobre o vetor $\delta_{0} \in l^{2}\left(\mathbb{Z}^{\nu}\right)$, o espaço das funções quadradosomáveis em $\mathbb{Z}^{\nu}$. Podemos então reescrever o hamiltoniano como $H=A+\lambda P$, onde $\lambda$ é independente de $A$ e distribuído de acordo com a lei $d P$. O trabalho mostra (Teorema 2) que se $d P$ for absolutamente contínua, uma condição suficiente para que o espectro seja puramente pontual (localização) é

$$
\lim _{\varepsilon \rightarrow 0} \sum_{x}|G(0, x ; E+i \varepsilon)|^{2}<\infty
$$

condição esta equivalente, portanto, a quadrado-somabilidade dos autovetores generalizados (que por sua vez está diretamente associada à existência de espectro pontual).

Os autores também deduzem o decaimento exponencial dos autovetores a partir do decaimento exponencial da função de Green (Teorema 9), complementando os resultados de [FS] apresentados acima.

Vale destacar que von Dreifus e Klein apresentaram em [vDK] uma demonstração muito mais simples, principalmente no que se refere a estimativas probabilísticas, do resultado original de Fröhlich e Spencer.

\section{Estados estendidos}

Como vimos acima, a questão da localização é bem compreendida e verificada não somente apenas no modelo de Anderson, mas em modelos desordenados gerais. Entretanto, a existência de espectro absolutamente contínuo para o modelo de Anderson "tightbinding" é até hoje um desafio matemático. Pelo que discutimos, a existência de estados generalizados estendidos está diretamente associada à presença desse tipo espectral. Esta associação foi posta em bases matemáticas mais sólidas por Wilcox [W], que definiu estados espalhados, isto é, estados que nos limites $t \rightarrow \pm \infty$ estão assintoticamente distantes do centro espalhador (a ligação com a expressão "estados estendidos" é óbvia), baseado diretamente no significado físico de espalhamento, desenvolvendo uma teoria abstrata de operadores de onda. Esbocemos suas principais idéias.

Seja $K \subset \mathbb{Z}^{\nu}$. A grandeza

$$
I(K, t)=\sum_{x \in K}\left|\psi_{t}(x)\right|^{2}
$$

é interpretada como a probabilidade do sistema (elétron em nosso caso) se encontrar no conjunto $K$ no instante de tempo $t$. O significado fundamental da afirmação " $\psi_{t}$ é uma onda espalhada" é que esta inevitavelmente escapa de qualquer conjunto limitado $K$ :

$$
\lim _{t \rightarrow \infty} I(K, t)=0
$$


para todo conjunto $K \subset \mathbb{Z}^{\nu}$ limitado, sendo portanto natural afirmar que $\psi_{0} \in \mathcal{H}$ é um estado espalhado (ou estendido) caso a relação (1.2) seja satisfeita.

A condição (1.2) pode ser generalizada ao contexto da teoria abstrata de espalhamento reformulando-a em termos de operadores; defina o operador $Q_{q}: \mathcal{H} \rightarrow \mathcal{H}$ através da expressão

$$
Q_{q} \psi_{t}(x)=\chi_{q}(x) \psi_{t}(x)
$$

para todo $x \in \mathbb{Z}^{\nu}, \chi_{q}$ a função característica do conjunto $B_{q}=\{x:|x| \leq|q|\}$. Desse modo $I\left(B_{q}, t\right)=\left\|Q_{q} U(t) \psi_{0}\right\|^{2}\left(U(t)=e^{-i t H}\right)$ e (1.2) é equivalente a

$$
\lim _{t \rightarrow \infty}\left\|Q_{q} U(t) \psi_{0}\right\|=0
$$

para todo $q \geq 0$.

Definimos o conjunto $\mathcal{H}_{\text {es }}$ como o conjunto de todos os estados espalhados de $H$ e o conjunto $\left\{Q_{q}: 0 \leq q<\infty\right\}$ de operadores que satisfazem (1.3). Um dos principais resultados de $[\mathrm{W}]$ nos diz que $\mathcal{H}_{\mathrm{es}} \subset \mathcal{H}_{\mathrm{c}}, \mathcal{H}_{\mathrm{c}}$ o subespaço dos estados associados à parte contínua do espectro (Teorema 2.1 de [W]; vide o Apêndice B para uma discussão completa da decomposição do espaço de Hilbert em diversos subespaços). Como sabemos, $\mathcal{H}_{\mathrm{c}}=$ $\mathcal{H}_{\mathrm{ac}} \oplus \mathcal{H}_{\mathrm{sc}}$ (Teorema B.1.18), $\mathcal{H}_{\mathrm{sc}}$ o subespaço dos estados singular-contínuos. Dessa forma, seria possível haver estados espalhados associados ao espectro singular-contínuo. Sob a hipótese de $Q_{q}$ ser $H$-compacto ${ }^{\dagger}$, é possível mostrar que $\mathcal{H}_{\mathrm{sc}}=\{0\}$ (Corolário 2.4 de $[\mathrm{W}])$.

Ao subespaço $\mathcal{H}_{\mathrm{sc}}$ geralmente não se atribuía nenhuma interpretação física (vide, por exemplo, p.23 de [RS4]). A primeira pessoa a fazê-lo foi Pearson [P], que construiu uma série de medidas espectrais singular-contínuas como limites de medidas absolutamente contínuas associadas a operadores com uma seqüência infinita de "barreiras", cujas distâncias entre si crescem rapidamente.

Os potenciais empregados por Pearson possuem simetria esférica, são limitados e localmente não-singulares. Na situação de decaimento, a condição para existência de espectro singular-contínuo é grosseiramente dada por $\sum_{n=1}^{\infty} g_{n}^{2}=\infty, g_{n}$ a altura da $n$-ésima barreira (veremos na Seção 3.3 uma condição mais precisa para os operadores que estudamos). Esta é exatamente a condição para a partícula (elétron, por exemplo), após um processo que envolve múltiplas reflexões e transmissões por sucessivas barreiras, retornar com probabilidade 1 a alguma vizinhança da origem (embora a partícula possa se encontrar a uma grande distância da origem, esta retornará a mesma com uma freqüência arbitrária). Dessa forma, tais potenciais devem ser interpretados fisicamente como uma barreira totalmente refletora, na qual a partícula pode penetrá-la arbitrariamente, sendo em algum momento refletida.

\footnotetext{
†Um operador $A$ é $B$-compacto se, dada uma seqüência $u_{n} \in D(A) \subset D(B), \operatorname{com}\left\{u_{n}\right\}$ e $\left\{A u_{n}\right\}$ limitadas, a seqüência $\left\{B u_{n}\right\}$ contém uma subseqüência limitada; vide p. 194 de [Ka].
} 
Tendo como base os resultados de Wilcox e Pearson, podemos interpretar fisicamente os espectros absolutamente contínuo e singular-contínuo como aqueles associados a estados espalhados (ou seja, cuja probabilidade de serem encontrados em uma região limitada do espaço é nula), os primeiros tendo uma probabilidade de recorrência à origem menor do que 1 (podendo se anular), os segundos retornado com probabilidade 1 . O cenário físico é, como descreve Pearson, o de uma partícula realizando uma espécie de caminhada aleatória quântica, na qual o efeito do potencial a distâncias pequenas não pode ser ignorado, por maior que seja a distância da partícula à origem. Assim, podemos de fato afirmar que estados efetivamente espalhados no sentido físico são aqueles associados somente à parte absolutamente contínua do espectro, já que estes são os estados em que a partícula descreve uma "caminhada aleatória" transiente.

\section{Modelos que exibem transição espectral}

Esses resultados validam em um certo sentido as aspirações de que a fase metálica (condutividade positiva) se relaciona direta e unicamente ao espectro absolutamente contínuo. A existência desse tipo de espectro é conhecida para alguns potenciais unidimensionais quase periódicos, como observado por Dinaburg e Sinai em [DS].

O chamado operador quase Mathieu (também conhecido como operador de Harper ou modelo de Hofstadter) é um belo exemplo de um operador unidimensional com um espectro bastante rico, podendo inclusive exibir uma transição do tipo metal-isolante (relacionada à transição do espectro absolutamente contínuo ao puramente pontual, com autovetores que decaem exponencialmente). Nele, definimos o potencial como $V(n)=$ $\lambda \cos (2 \pi \alpha n+\theta)$, onde $n \in \mathbb{Z}$ é um ponto qualquer da rede unidimensional e $\alpha, \lambda, \theta \in \mathbb{R}$. O operador $H_{\alpha, \lambda, \theta}:=-\Delta+V$ é um modelo "tight-binding" para o hamiltoniano de um elétron sujeito a um potencial comensurável (se $\alpha$ é racional) ou incomensurável (caso $\alpha$ seja irracional), e desempenha um papel importante no estudo de problemas fundamentais relacionados a elétrons de Bloch na presença de campos magnéticos, principalmente na teoria de Thouless, Kohmoto, Nightingale e den Nijs do efeito Hall quântico inteiro [TKNN], recentemente verificada experimentalmente (vide [L2] para maiores detalhes e mais aplicações físicas do operador de Mathieu).

Em particular, para o caso periódico $(\alpha \in \mathbb{Q}), H_{\alpha, \lambda, \theta}$, como qualquer potencial periódico, possui espectro puramente absolutamente contínuo. Para $\alpha$ irracional, no entanto, a natureza espectral de $H_{\alpha, \lambda, \theta}$ é bem mais complexa e depende de valores precisos dos parâmetros. Um dos pricipais resultados é o Teorema 3.4 presente em [L2] (vide bibliografia complementar), que afirma que para quase todo par de parâmetros $\alpha, \theta$ com respeito à medida de Lebesgue (ou seja, a menos de um conjunto de medida de Lebesgue nula), o espectro é absolutamente contínuo quando $|\lambda|<2$, puramente singular-contínuo quando $\lambda=2$ e puramente pontual denso (isto é, o espectro é um conjunto denso em $\mathbb{R}$; vide o Apêndice B para a definição desse tipo espectral), com autovetores que decaem exponencialmente (estados localizados) quando $|\lambda|>2$. Há, portanto, a transição metal-isolante 
destacada anteriormente. O resultado acima é válido do ponto de vista probabilístico, já que exclui conjuntos de pontos com probabilidade nula (medida de Lebesgue nula); o cenário completo é bem mais delicado, pois tais conjuntos de medida nula originam diferentes propriedades espectrais.

O modelo de Anderson na rede de Bethe com desordem fraca é outro importante exemplo de existência de espectro absolutamente contínuo em operadores aleatórios. O resultado foi obtido por Klein em [K] e posteriormente por Froese, Hasler e Spitzer em [FHS2], através de uma abordagem bastante diferente.

Os operadores de Schrödinger com potenciais esparsos constituem outro exemplo de operadores em que é possível existir transição espectral. Potenciais esparsos são nulos fora de uma seqüência de "barreiras" cujas distâncias entre si crescem rapidamente à medida que se afastam da origem. As barreiras em si podem possuir "tamanhos" distintos, que ou crescem, ou diminuem, ou permanecem inalterados.

Podemos, por exemplo, definir uma classe de operadores discretos unidimensionais em $l^{2}(\mathbb{N}, \mathbb{C})$, cujo potencial $V=0$ se $n \notin\left\{a_{j}\right\}_{j=1}^{\infty}$. O conjunto $\left\{a_{j}\right\}_{j=1}^{\infty}$ é uma seqüência rapidamente crescente de números naturais, com $V\left(a_{j}\right)$ números reais distintos de zero. A taxa de crescimento da seqüência $\left\{a_{j}\right\}_{j=1}^{\infty}$ é em princípio arbitrária, sendo no mínimo tão rápida quanto a linear.

Como vimos anteriormente, Pearson $[\mathrm{P}]$ foi o primeiro a reconhecer a utilidade deste tipo de potencial na construção de operadores com espectro singular-contínuo e a atribuir uma interpretação física a este tipo espectral. Apesar desses operadores aparentemente não apresentarem uma conexão direta com problemas físicos, estes são um bom laboratório para o estudo mais detalhado dos vários tipos espectrais, uma vez que a relativa facilidade em analisá-los nos permite um conhecimento detalhado dos autovetores generalizados.

Um dos principais resultados da área se deve a Kiselev, Last e Simon [KLS], que demonstraram que nos limites $a_{j} / a_{j+1} \rightarrow 0$ e $V\left(a_{j}\right) \rightarrow 0(j \rightarrow \infty), H$ possui espectro absolutamente contínuo caso o potencial seja quadrado-somável e espectro puramente singular-contínuo caso contrário (Teorema 1.7 de [KLS]).

Gordon [G] e Kirsch, Molchanov e Pastur [KMP] usaram potenciais esparsos crescentes para construir operadores de Schrödinger determinísticos com espectro puramente pontual denso. O que eles mostraram, essencialmente, é que para uma dada distribuição de barreiras $\left\{a_{j}\right\}_{j=1}^{\infty}$, é possível tomar $\left\{V\left(a_{j}\right)\right\}_{j=1}^{\infty}$ com um crescimento de tal modo que $H$ possua espectro puramente pontual para quase toda condição de contorno $\theta$. Outros modelos desse tipo foram estudados por Last e Simon [LS2], que demonstraram que para $a_{j}=j^{2}$ e $V\left(a_{j}\right)=e^{c j}, c>0, H$ possui espectro puramente pontual no intervalo $[-2,2]$ para quase toda condição de contorno $\theta$, com os autovetores decaíndo como $e^{-c n / 2}$.

No sentido contrário, Simon e Stolz [SS] demonstraram que para uma seqüência ilimitada dos valores do potencial $\left\{V\left(a_{j}\right)\right\}_{j=1}^{\infty}$, é possível escolher uma seqüência de barreiras que cresça rápido o suficiente de tal modo que $H$ contenha apenas espectro singularcontínuo em $(-2,2)$ para toda a condição de contorno.

Vale notar que o espectro absolutamente contínuo não se faz presente nessa situação 
de operadores esparsos ilimitados, como Simon e Spencer demonstraram em [SiSp] (vide [LS1] para outra demonstração; Deift e Killip [DK] demonstraram um resultado análogo para potenciais não necessariamente esparsos). Assim, o espectro é sempre singular e depende diretamente das taxas de crescimento das seqüências $\left\{a_{j}\right\}_{j=1}^{\infty}$ e $\left\{V\left(a_{j}\right)\right\}_{j=1}^{\infty}$ : um crescimento mais acelerado da esparsidade torna o espectro mais "contínuo", enquanto que um crescimento maior do potencial o torna mais "singular" (estes conceitos são tratados com precisão quando introduzimos o conceito de dimensão Hausdorff de conjuntos e medidas de Borel; para tanto, vide o Apêndice G).

De fato, Zlatoš foi capaz de demonstrar em [Z] a existência de uma transição entre espectros puramente pontual e singular-contínuo para uma classe de operadores nos quais $\left\{V\left(a_{j}\right)\right\}_{j=1}^{\infty}$ é uma seqüência constante e $\left\{a_{j}\right\}_{j=1}^{\infty}$ é uma seqüência de variáveis aleatórias uniformemente distribuídas que cresce exponencialmente (os detalhes se encontram mais adiante). Ele mostrou que dependendo da taxa de esparsidade e da intensidade das barreiras, é possível haver ou espectro puramente pontual ou espectro singular-contínuo, este com natureza distinta à medida que nos deslocamos no espectro. Repare que nesse modelo a indeterminação do potencial se encontra nas posições das barreiras, algo ligeiramente diferente do que se geralmente se estuda em operadores de Schrödinger aleatórios.

Um modelo bastante similar a este foi proposto por Marchetti et. al. em [MWGA], em que as perturbações, além de determinísticas, são atribuídas a termos não-diagonais do laplaciano discreto, diferentemente dos modelos unidimensionais discutidos até então.

A saber,

$$
\left(-\Delta_{P} u\right)(n)=p_{n} u(n+1)+p_{n-1} u(n-1),
$$

em que $0<p_{n} \leq 1$ representa o peso associado à ligação entre os sítios $n$ e $n+1$. Note que $p_{n}=1$ é o análogo a $V(n)=0$ nos modelos anteriores. O operador satisfaz

$$
u(-1) \cos \phi-u(0) \sin \phi=0
$$

como condição de contorno $\phi \in[0, \pi)$ no ponto $n=-1$ (o modelo é definido em $\left.\mathbb{Z}_{+} \equiv\{0,1,2, \ldots\}\right)$. É possível mostrar que esta atribuição de perturbação não produz diferenças, tanto nos tipos espectrais quanto nas transições entre eles, com respeito ao modelo de Zlatoš. A vantagem está no fato de se poder efetuar uma grande variação na intensidade das barreiras através de uma pequena variação no valor de $p_{n}(V(n)$ percorre o intervalo $[0, \infty)$ à medida que $p_{n}$ percorre o intervalo $(0,1]$, de 1 a 0$)$.

Observação 1.1 Vale destacar que denotamos por $\Delta$ o laplaciano discreto, com uma diferença de sinal em relação à notação anterior. O operador analisado é o mesmo.

É interessante notar que mais uma vez a ausência de espectro absolutamente contínuo em ambos os modelos. Uma justificativa plausível é, como sabemos do Teorema 1.1 de [DK], o fato do potencial, apesar de esparso e limitado, não ser quadrado-somável. Uma demonstração efetiva desse fato se encontra em [MWGA] (Teorema 4.4), onde os autores usam alguns resultados (discutidos em detalhes na Seção F.2) desenvolvidos por Last e Simon em [LS1] à determinação da natureza espectral. 


\section{$1.2 \quad$ Modelo estudado}

Por tudo aquilo que vimos até o momento, são muito poucos os modelos cujos operadores associados apresentam um espectro absolutamente contínuo, e ainda menos numerosos aqueles com uma transição ao espectro puramente pontual (o que eventualmente representaria uma transição do tipo metal-isolante). Podemos até dizer que não existe nenhum modelo unidimensional com potencial esparso que apresente esta transição.

A aparente estabilidade do espectro absolutamente contínuo em modelos com estruturas multidimensionais (como a árvore de Cayley, por exemplo) nos leva a questionar a possibilidade de se propor um modelo de simples análise, do tipo potencial esparso, em mais dimensões, em que não só o espectro absolutamente contínuo seja parcialmente estável como também exista uma transição entre este tipo espectral e o tipo puramente pontual. Um dos principais objetivos desse trabalho é justamente apresentar um modelo que atenda esses requisitos.

A extensão mais pertinente a se adotar em nossa opinião é a do modelo proposto por Marchetti et. al. (expresso por (1.4)) para uma faixa $\Lambda=\mathbb{Z}_{+} \times\{0,1,2, \ldots, L-1\}$ de comprimento $L$ no plano $\mathbb{Z}_{+}^{2}$. A saber, definimos no espaço de Hilbert $l^{2}\left(\Lambda, \mathbb{C}^{L}\right)$ o operador aleatório $\prod_{P, \phi}^{\omega}$ para cada seqüência $P=\left(p_{n}(\omega)\right)_{n \geq-1}$ de números aleatórios $p_{n}(\omega) \in(0,1)$, a partir da equação

$$
\left(\Pi_{P, \phi}^{\omega} u\right)(k, m):=p_{k}(\omega) u(k+1, m)+p_{k-1}(\omega) u(k-1, m)+u(k, m+1)+u(k, m-1),
$$

válida para toda dupla $(k, m) \in \Lambda$, satisfazendo a condição de contorno $(\phi \in[0, \pi))$

$$
u(-1, m) \cos \phi-u(0, m) \sin \phi=0
$$

para cada $m \in\{0, \ldots, L-1\}$, e condições periódicas na direção vertical:

$$
u(k, L)=u(k, 0)
$$

para todo $k \in \mathbb{Z}_{+} ; \omega=\left(\omega_{1}, \omega_{2}, \ldots\right)$ representa uma seqüência de variáveis aleatórias independentes definidas em um espaço de probabilidade $(\Xi, \mathcal{B}, \mu)$, uniformemente distribuídas nos conjuntos $\Gamma_{j} \equiv\{-j, \ldots, j\}$.

Repare na independência entre as duas direções no modelo definido por (1.6): na direção de $\mathbb{Z}_{+}$atua o operador unidimensional definido por (1.4), enquanto que na direção ortogonal atua o laplaciano discreto, sem perturbações. Veremos a seguir que é possível representar o operador $\Pi_{P, \phi}^{\omega}$ como uma soma de Kronecker entre uma matriz de Jacobi, associada ao operador definido em $\mathbb{Z}_{+}$, e uma matriz de diferença finita, associada ao laplaciano em $\{0,1, \ldots, L-1\}$. Veremos também que essa ausência de interação bidimensional resulta em uma fatorização tanto do espectro quanto dos autovetores: a medida espectral matricial $\Omega$ associada a $\Pi_{P, \phi}^{\omega}$ é tal que seus constituintes podem ser escritos como o produto de convolução das medidas associadas a cada componente unidimensional, assim como seus autovetores generalizados são o produto de Kronecker dos autovetores associados aos operadores unidimensionais (os detalhes se encontram na Seção 2.3). 
Denotamos por quase-unidimensional um modelo com tais características, já que estas (principalmente as espectrais) diferem muito pouco daquelas do modelo unidimensional relacionado.

O operador $\Pi_{P, 0}^{\omega}$ com condição de contorno $\phi=0$ é, em particular, definido em um cilindro tal que $u(-1, m)=0$ para todo $m \in\{0, \ldots, L-1\}$; o operador $\Pi_{0, \phi}$, definido adotando-se $p_{n} \equiv 1$ para todo $n$, é equivalente ao laplaciano usual em $\Lambda$ com condição de contorno $\phi$. Vale destacar mais uma vez que $p_{k}(\omega)$ se refere ao peso associado à ligação horizontal $\langle(k, m),(k+1, m)\rangle, m \in\{0, \ldots, L-1\}$.

Definição 1.2 Dizemos que o operador $\prod_{P, \phi}^{\omega}$ é homogeneamente transversal se, para qualquer realização de $\omega,(1.6)$ é definido com o mesmo valor $p_{k}(\omega)$ para cada $m$.

É possível considerar outro tipo de perturbação por técnicas um pouco distintas daquelas empregadas neste trabalho para o estudo da faixa. Abordaremos este tema quando discutirmos o modelo estendido ao plano.

A seqüência $P=\left(p_{n}(\omega)\right)_{n \geq-1}$ de barreiras é da forma

$$
p_{n}=\left\{\begin{array}{cll}
1-\delta & \text { se } & n=a_{j}^{\omega} \in \mathcal{A}, \\
1 & \text { se } & n \notin \mathcal{A}
\end{array}\right.
$$

em que $\delta \in(0,1)$ e $\mathcal{A}=\left(a_{j}^{\omega}\right)_{j \geq 1}$ é um conjunto aleatório de números naturais $a_{j}^{\omega}=a_{j}+\omega_{j}$ tal que $a_{j}$ satisfaz

$$
a_{j}-a_{j-1} \geq 2, \quad j=2,3, \ldots
$$

e

$$
\lim _{j \rightarrow \infty} \frac{a_{j+1}}{a_{j}}=\beta>1
$$

A condição (1.10) torna cada barreira localizada em uma única coluna de ligações verticais, com $\beta$ o dito "parâmetro de esparsidade". Fixamos, como em [MWGA], a separação entre as barreiras a partir da identidade

$$
a_{j}-a_{j-1}=\beta^{j}, \quad j=2,3, \ldots
$$

com $a_{1}+1=\beta \geq 2$ um inteiro, visando simplificar nossa análise ${ }^{\dagger}$. Repare que à medida que a distância entre os valores médios (na distribuição uniforme $\mu_{j}$ ) das barreiras cresce exponencialmente (condição (1.11)), a incerteza associada às suas posições cresce linearmente com o índice de esparsidade, de tal forma que não é possível haver sobreposições entre elas. Essa escolha é a mesma feita por Zlatoš, não sendo necessária aos resultados que obtemos; como veremos adiante (Seção 3.2), basta um crescimento com qualquer potência positiva na incerteza da posição das barreiras.

Consideramos neste trabalho somente seqüências na família a três parâmetros $\mathcal{P}_{\delta, \beta}^{\omega}$, definida por (1.9), com $\mathcal{A}$ expresso por (1.11), e denotamos por $\Pi_{\delta, \phi}$ o operador correspondente com $P=\left\{p_{n}\right\}_{n \geq-1}$ dessa forma.

\footnotetext{
${ }^{\dagger}$ Poderíamos definir $\beta$ como um número real maior que 1 e reescrever (1.11) como $a_{j}-a_{j-1}=\left[\beta^{j}\right]$, $j=2,3, \ldots$, em que definimos $[x]$ como sendo a parte inteira de $x \in \mathbb{R}$, isto é, $[x]:=\max \{y \in \mathbb{Z}: y \leq x\}$.
} 
Observação 1.3 Omitiremos o índice $\omega$ no decorrer do texto a fim de não carregar a notação, sendo subentendida a dependência em $\omega$ de qualquer operador ou função de $p_{n}$. A exceção é feita quando a distinção entre os casos puramente determinístico e aleatório é necessária.

\section{Matrizes bloco--Jacobi}

Como mencionado anteriormente, podemos representar o operador $\Pi_{P, 0}$ através da matriz bloco-Jacobi

$$
\mathcal{J}_{P}=J_{P} \otimes I_{L}+I \otimes A_{L},
$$

sujeita à condição de contorno de Dirichlet $u(-1, m)=0, m \in\{0,1, \ldots L-1\}$, e à condição periódica $u(k, 0)=u(k, L-1)$, com $p_{-1}=1$ fixo. $I$ representa o operador identidade em $l^{2}\left(\mathbb{Z}_{+}, \mathbb{C}\right)$ e $J_{P}$ a matriz de Jacobi definida por

$$
J_{P}=\left(\begin{array}{ccccc}
0 & p_{0} & 0 & 0 & \cdots \\
p_{0} & 0 & p_{1} & 0 & \cdots \\
0 & p_{1} & 0 & p_{2} & \cdots \\
0 & 0 & p_{2} & 0 & \cdots \\
\vdots & \vdots & \vdots & \vdots & \ddots
\end{array}\right)
$$

$\left(\left(p_{n}\right)_{n \geq-1}\right.$ como em (1.9)), $A_{L}$ a matriz de diferença finita $L \times L$

$$
A_{L}=\left(\begin{array}{cccccc}
0 & 1 & 0 & \cdots & 0 & 1 \\
1 & 0 & 1 & \cdots & 0 & 0 \\
\vdots & \vdots & \vdots & \ddots & \vdots & \vdots \\
0 & 0 & 0 & \cdots & 0 & 1 \\
1 & 0 & 0 & \cdots & 1 & 0
\end{array}\right)
$$

e $I_{L}$ a matriz identidade $L \times L$.

Observação 1.4 1. Identificamos o operador $\Pi_{\delta, 0}$ e a matriz bloco-Jacobi $\mathcal{J}_{\delta}$, de tal forma que qualquer propriedade espectral de $\Pi_{\delta, 0}$ e de $\mathcal{J}_{\delta}$ são equivalentes.

2. O operador $\Pi_{\delta, \phi}$, com $\phi \neq 0$, também pode ser expresso como uma matriz blocoJacobi. Se $\mathcal{J}_{\delta, \phi}$ denota a matriz correspondente, segue que

$$
\mathcal{J}_{\delta, \phi}=\mathcal{J}_{\delta}+E_{0} \otimes \tan \phi I_{L}
$$

onde $E_{0}$ representa em $l^{2}\left(\mathbb{Z}_{+}, \mathbb{C}\right)$ o operador cujos elementos são todos nulos exceto $\left(E_{0}\right)_{00}=1$. Trata-se, portanto, de uma perturbação de posto um da matriz $\mathcal{J}_{\delta}$, o que preserva seu espectro essencial (Definição B.2.8). Se fizemos $\phi$ variar com $m$, basta substituirmos $\tan \phi I_{L}$ por $\operatorname{diag}\left\{\tan \phi_{m}\right\}_{m=0}^{L-1}$ em (1.15). Esta é exatamente a representação do operador $\Pi_{\delta, \phi}$ em uma soma de Kronecker que discutimos anteriormente. 
Vale destacar que Marchetti et. al. estudaram em [MWGA] uma classe de operadores determinísticos (independentes de $\omega$ ) equivalentes ao caso particular $L=1$ (em que $I_{L}=1$ e $\left.A_{L}=0\right)$.

É interessante notar que à medida que o parâmetro $\delta$ percorre o intervalo $(0,1)$, a matriz de Jacobi $J_{\delta, \phi}$ interpola continuamente duas situações: um espectro puramente pontual caso $\delta=1\left(J_{\delta, \phi}\right.$ pode ser expressa como a soma direta de matrizes de dimensão finita, que como sabemos, possuem espectro puramente pontual) e um espectro absolutamente contínuo caso $\delta=0\left(J_{\delta, \phi}\right.$ é simplesmente a representação matricial do laplaciano discreto, cujo espectro é absolutamente contínuo).

Uma discussão de uma classe maior de perturbações não tratadas nesse trabalho se encontra na Seção 1 de [MWGA]. Em particular, Marchetti et. al. demonstraram que para a classe de matrizes de Jacobi satisfazendo a condição de esparsidade (1.10), com $\lim _{n \rightarrow \infty} p_{n}=1$, o espectro é puramente absolutamente contínuo caso $\sum\left|1-p_{n}\right|^{2}<\infty$ e puramente singular-contínuo caso contrário, com o parâmetro de esparsidade $\beta=\infty$ (Teorema 1.1; a demonstração deste resultado segue as linhas do Teorema 1.7 de [KLS]). O que esse resultado nos diz é que não existe transição espectral para essa classe de matrizes de Jacobi, de modo que a condição para que exista uma transição, muito provavelmente entre espectros singular-contínuo e puramente pontual, é que as barreiras se mantenham finitas no limite $n \rightarrow \infty$, condição satisfeita pelo modelo que estudamos. Veremos (Seção 3.3) que uma mediação entre esparsidade e perturbação pode produzir uma transição espectral (novamente entre os espectros singulares) numa situação de esparsidade com crescimento geométrico (como expresso por (1.11)).

Sendo a medida espectral associada à matriz bloco-Jacobi $\mathcal{J}_{\delta, \phi}$ (a representação matricial do operador $\Pi_{\delta, \phi}$, como discutido anteriormente) o produto de convolução das medidas espectrais das matrizes $J_{\delta, \phi}$ e $A_{L}$, torna- se claro que esta também é singular, já que a convolução de uma medida singular (contínua ou discreta) com uma medida discreta é singular (exercício 6 da Seção 6.1 de [C]). Dessa forma, nosso desejo de obter o espectro absolutamente contínuo foi frustrado no modelo definido na faixa finita. No entanto, tanto o espectro quanto a medida espectral desse operador apresentam propriedades extremamente interessantes, que acabam nos motivando a estudá-lo.

\section{Modelos definidos no plano $\mathbb{Z}_{+}^{2}$}

Os resultados são muito mais animadores para alguns modelos esparsos definidos no plano $\mathbb{Z}_{+}^{2} \equiv \mathbb{Z}_{+} \times \mathbb{Z}_{+}$. Denotamos por $\Pi_{P, \phi, \psi}^{\omega}$ o operador representado pela equação (1.6) e pelas condições de contorno

$$
u(-1, m) \cos \phi-u(0, m) \sin \phi=0
$$

$(\phi \in[0, \pi))$ para todo $m \in \mathbb{Z}_{+}, \mathrm{e}$

$$
u(k,-1) \cos \psi-u(k, 0) \sin \psi=0
$$


$(\psi \in[0, \pi))$ para todo $k \in \mathbb{Z}_{+}$. A seqüência de barreiras $P$ é definida em analogia a (1.9), satisfazendo inclusive a mesma condição de esparsidade.

Trata-se, portanto, de uma extensão direta do modelo estudado na faixa $\Lambda$ para o plano $\mathbb{Z}_{+}^{2}$. Repare que a matriz de diferença finita é substituída pelo laplaciano livre com condição de contorno $\psi$, em detrimento a uma condição de contorno periódica.

Assim como com o operador $\Pi_{P, 0}$ (que satisfaz a condição de Dirichlet em $k=-1$ ), podemos representar o operador $\Pi_{P, 0,0}$ através da matriz bloco-Jacobi

$$
\mathcal{J}_{P, 0}=J_{P} \otimes I+I \otimes J_{0},
$$

com $I$ o operador identidade em $l^{2}\left(\mathbb{Z}_{+}, \mathbb{C}\right), J_{P}$ e $J_{0}$ as matrizes de Jacobi dadas por (1.13) $\left(p_{i}=1\right.$ para todo $i$ na definição de $J_{0}$, a chamada matriz de Jacobi livre).

Observação 1.5 A extensão dos resultados discutidos na Observação 1.4 é imediata: podemos representar o operador $\Pi_{P, \phi, \psi}$, com $\phi, \psi \neq 0$, como uma matriz bloco-Jacobi. A saber,

$$
\mathcal{J}_{P, \phi, \psi}=\mathcal{J}_{P, 0}+E_{0} \otimes \tan \phi I+\tan \psi I \otimes E_{0},
$$

onde $E_{0}$ novamente representa em $l^{2}\left(\mathbb{Z}_{+}, \mathbb{C}\right)$ o operador cujos elementos são todos nulos exceto $\left(E_{0}\right)_{00}=1$.

É possivel mostrar (vide a Subseção 2.4.1) que o espectro do laplaciano livre é absolutamente contínuo, independentemente da condição de contorno (o que é equivalente ao suporte mínimo ${ }^{\dagger}$ da parte absolutamente contínua do espectro ser independente da condição de contorno; vide o Lema D.4.3). Como a medida espectral matricial de $\mathcal{J}_{P, \phi, \psi}$ também é definida de modo que seus constituíntes são obtidos como o produto de convolução das medidas espectrais de $J_{P, \phi}$, singular, e de $J_{0, \psi}$, absolutamente contínua, concluímos que o espectro essencial de $\mathcal{J}_{P, \phi, \psi}$ é puramente absolutamente contínuo (vide mais uma vez exercício 6 da Seção 6.1 de [C]). Apesar de termos apresentado um modelo com espectro absolutamente contínuo, nele não detectamos a transição que desejamos entre este tipo espectral e o puramente pontual (de fato, não detectamos qualquer tipo de transição).

Sugerimos então outro modelo, representado pelo operador

$$
\begin{aligned}
\left(\Pi_{P, Q, \phi, \psi}^{\omega, \nu} u\right)(k, m) & :=p_{k}(\omega) u(k+1, m)+p_{k-1}(\omega) u(k-1, m)+q_{m}(\nu) u(k, m+1) \\
& +q_{m-1}(\nu) u(k, m-1),
\end{aligned}
$$

que satisfaz as mesmas condições de contorno (1.16) e (1.17) do operador $\prod_{P, \phi, \psi}^{\omega}$. A diferença entre este modelo e o anterior reside no fato de inserirmos barreiras, mais uma vez esparsas, em ambas as direções. As seqüências de barreiras $P$ e $Q$ são definidas em analogia a (1.9), com distintas "alturas" $\delta$ e distintos parâmetros de esparsidade $\beta$. Novamente $\omega=\left(\omega_{1}, \omega_{2}, \ldots\right)$ e $\nu=\left(\nu_{1}, \nu_{2}, \ldots\right)$ representam seqüências de variáveis aleatórias independentes definidas novamente no espaço de probabilidade $(\Xi, \mathcal{B}, \mu)$, uniformemente distribuídas nos conjuntos $\Gamma_{j} \equiv\{-j, \ldots, j\}$.

\footnotetext{
${ }^{\dagger}$ vide a Definição D.3.7.
} 
Podemos novamente representar o operador $\Pi_{P, Q, \phi, \psi}^{\omega, \nu}$ através da matriz bloco-Jacobi

$$
\mathcal{J}_{P, Q, \phi, \psi}=J_{P, \phi} \otimes I+I \otimes J_{Q, \psi}
$$

expressa como a soma de Kronecker das matrizes de Jacobi $J_{P}$ e $J_{Q}$ dadas por (1.13) (substitui-se $p_{i}$ por $q_{i}$ ao se definir $J_{Q}$ ). A matriz $\mathcal{J}_{P, Q, \phi, \psi}$ é mais uma vez uma perturbação de posto finito (mais precisamente, de posto 2 ) da matriz $\mathcal{J}_{P, Q, 0,0}$ com condições de contorno de Dirichlet, exatamente como o resultado expresso na Observação 1.5.

Como veremos na Seção 3.3, dependendo da escolha das seqüências de barreiras $P$ e $Q$ e das condições de contorno $\phi$ e $\psi$, é possível construir matrizes de Jacobi $J_{P, \phi}$ e $J_{Q, \psi}$ que exibam uma transição de espectro singular-contínuo a puramente pontual. Uma vez que a convolução de medidas singular-contínuas pode resultar em uma medida absolutamente contínua (vide exercício 9 da Seção 6.3 de [C] para um exemplo) e a convolução de medidas puramente pontuais é sempre puramente pontual, o modelo possibilita a transição do espectro absolutamente contínuo ao puramente pontual. Acreditamos que esta transição não é obrigatoriamente aguda, passando por uma parte de espectro singular-contínuo.

Chegamos, portanto ao modelo que desejávamos. Vale destacar que a idéia de estudar a soma de Kronecker de matrizes de Jacobi com perturbações esparsas é, em certo sentido, uma generalização da idéia de Malozemov e Molchanov (vide [Si1]), que perceberam a possibilidade da existência da transição do espectro absolutamente contínuo a puramente pontual ao se convoluir medidas espectrais singulares associadas a modelos unidimensionais.

\section{Transformada de Fourier da medida espectral}

Acreditamos que a melhor forma de se avaliar a possibilidade de existência de espectro absolutamente contínuo é através do comportamento assintótico (com o tempo) da transformada de Fourier da medida espectral, definida pela expressão

$$
\hat{\mu}_{\psi}(t)=\frac{1}{\pi} \int_{0}^{\pi} e^{-2 i t \cos k} d \mu_{\psi}(2 \cos k)
$$

( $\lambda=2 \cos k$ representa uma possível parametrização do espectro de $J_{P, \phi}$, como definido em (1.4); vide a Subseção 2.4.1 para a demonstração), diretamente relacionada (através de seu módulo-quadrado) à "probabilidade de sobrevivência", que estabelece a probabilidade de se encontrar a partícula (elétron, por exemplo) em seu estado inicial decorrido um intervalo de tempo $t$. Se denotarmos por $\psi$ o estado inicial da partícula e por $\left|\hat{\mu}_{\psi}(t)\right|^{2}$ a "probabilidade de sobrevivência", então temos a identidade $\left|\hat{\mu}_{\psi}(t)\right|^{2}=\left\|e^{-i t H} \psi\right\|^{2}$, onde $H$ denota o hamiltoniano (operador de Schrödinger) do modelo associado.

Isso porque compreendemos bem a conexão entre o comportamento assintótico de $\left|\hat{\mu}_{\psi}(t)\right|^{2}$ e os tipos espectrais. A saber, o chamado Lema de Riemann-Lebesgue (Teorema 2.1 de [L1]) nos diz que se a medida espectral $\mu_{\psi}$ é absolutamente contínua, então $\lim _{t \rightarrow \infty} \hat{\mu}_{\psi}(t)=0$ (o que garante uma condição necessária para existência de espectro 
absolutamente contínuo). Outro importante resultado é o Teorema de Wiener (Teorema XI.114 de [RS3]), que demonstra que $\lim _{t \rightarrow \infty} 1 / T \int_{0}^{T}\left|\hat{\mu}_{\psi}(t)\right|^{2} d t=\sum_{x \in \mathbb{R}}\left|\mu_{\psi}(\{x\})\right|^{2}$, ou seja, que a média temporal da transformada de Fourier da medida espectral é igual a soma de seus átomos (pontos com medida espectral positiva). Esse resultado implica, em particular, na exclusão da parte puramente pontual do espectro caso a média temporal apresentada acima seja assintoticamente nula.

Uma vez que a transformada de Fourier da medida espectral é, como vimos, um parâmetro ligado à dinâmica da partícula (evolução temporal do pacote de onda), somos mais uma vez remetidos à conexão existente entre tipos espectrais e os estados ligados ou estendidos.

Vale destacar que o Lema de Riemann-Lebesgue não garante uma condição suficiente à existência de espectro absolutamente contínuo, o que não nos permite concluir se a transformada de Fourier de medidas singular-contínuas tende ou não a zero no limite $t \rightarrow \infty$.

Alguns resultados ligeiramente diferentes caracterizam certos subespaços como fechos ${ }^{\dagger}$ de conjuntos de vetores com certas propriedades dinâmicas. Um deles, associado a Kato (vide [L1]), afirma que

$$
\mathcal{H}_{\mathrm{ac}}:=\left\{\varphi \in \mathcal{H} \mid \mu_{\varphi} \text { é absolutamente contínuo }\right\}=\overline{\left\{\varphi \in \mathcal{H} \mid \widehat{\mu}_{\varphi}(t) \in L^{2}\right\}} .
$$

Voltemos ao nosso problema em específico. Segue do Teorema da convolução (vide [C]) que a transformada de Fourier do produto de convolução de duas medidas é igual ao produto das suas transformadas. Como a medida espectral matricial de $\mathcal{J}_{P, Q, \phi, \psi}$ é desse tipo (vide o Capítulo 2.3 para a demonstração dessa afirmação), é possível que existam valores de energia (localizados no centro do espectro) para os quais sua transformada de Fourier seja quadrado-integrável, desde que asseguremos um decaimento grande o suficiente das medidas referentes às matrizes de Jacobi $J_{P, \phi}$ e $J_{Q, \psi}$. Por conseguinte, um conhecimento detalhado das transformadas de Fourier dessas medidas, aliado a (1.20), é mais do que suficiente para a determinação de uma transição espectral como discutida acima.

Os poucos artigos [KR, Si1] que analisam a questão da transformada de Fourier de modelos unidimensionais semelhantes aos estudados aqui são destinados a situações de esparsidades super-geométricas e muito pouco claros. Nossa idéia é tentar conectar de alguma forma o comportamento assintótico da transformada da medida às suas propriedades dimensionais, que como veremos são exatamente conhecidas. Este problema em si é deveras interessante, e pelo que sabemos, inédito. Existem alguns trabalhos, como o de Strichartz [Str], que relacionam a média de Cèsaro (média temporal) da transformada de Fourier a essas propriedades (vide [L1] para detalhes).

\footnotetext{
${ }^{\dagger}$ Um ponto $x$ é chamado de ponto de acumulação do conjunto $A$ se dada qualquer $\delta$-vizinhança de $x$ existir ao menos um $y \in A, y \neq x$; o fecho de $A$ é a união entre os pontos de $A$ e seus pontos de acumulação.
} 


\section{Dimensão Hausdorff}

A propriedade dimensional nos referimos à chamada dimensão Hausdorff, também conhecida como dimensão fractal, da medida espectral. Esta é um indicativo do grau de "singularidade" da medida espectral, sendo igual a 1 (menos singular possível) quando restrita à parte absolutamente contínua, 0 (mais singular possível) quando restrita à parte puramente pontual e qualquer número entre 0 e 1 quando restrita à parte singularcontínua do espectro (vide o Apêndice G para os detalhes). Existem inclusive exemplos de medidas singular-contínuas com dimensão Hausdorff igual a 0 ou 1 (vide o Capítulo 5 para alguns desses exemplos).

Jitomirskaya e Last, no seminal trabalho [JL], desenvolveram uma teoria que torna possível a caracterização precisa da parte singular-contínua do espectro. Ambos estenderam o conceito de subordinância, criado por Gilbert e Pearson em [GP], para medidas de Hausdorff, relacionando o comportamento assintótico dos autovetores, ou autofunções, da equação de Schrödinger a questões de continuidade e singularidade da medida espectral (diretamente ligadas à definição de dimensão Hausdorff; vide o Apêndice H para os detalhes).

Dessa forma, quando afirmamos que existe uma conexão entre o decaimento da transformada de Fourier da medida espectral e sua dimensão Hausdorff, dizemos implicitamente que o comportamento assintótico de $\hat{\mu}_{\varphi}(t)$ é diretamente relacionado ao comportamento assintótico dos autovetores. Uma vez que o decaimento da transformada depende do tipo espectral, concluímos que cada tipo espectral é definido pelos diferentes comportamentos assintóticos assumidos pelos autovetores. Essa conclusão, óbvia em certo sentido, é a base da teoria de subordinância de Gilbert e Pearson.

A vantagem em se estudar os modelos sugeridos por Zlatoš e Marchetti et. al. está no fato de podermos determinar com precisão arbitrária a dimensão Hausdorff local das respectivas medidas espectrais (o que Zlatoš de fato faz para o modelo com perturbações aleatórias; vide Teorema 6.3 de [Z]) dos operadores associados, e assim obter, como pretendemos, o comportamento assintótico de suas transformadas de Fourier.

\section{Principais resultados obtidos}

Apresentamos a seguir uma relação dos principais resultados obtidos nesse trabalho, comparando-os com os resultados análogos presentes na literatura.

Verificamos que apesar de essencialmente unidimensional, o modelo definido a partir da relação (2.4.1) apresenta algumas diferenças marcantes em relação ao modelo definido por (1.4), seu análogo unidimensional. A principal delas se evidencia na medida espectral, uma matriz $L \times L$, que incorpora a multiplicidade intrínseca ao modelo. O fato desta matriz ser absolutamente contínua com respeito a "medida de mistura" $\frac{1}{L} \sum_{j=0}^{L-1} \mu_{j}, \mu_{j}$ a medida associada à matriz de Jacobi $J_{\delta, \phi}^{j}$ (vide a Seção 2.3, em particular a Proposição 2.3.2), nos permite identificar as principais características espectrais de (2.4.1) a partir 
do estudo do comportamento coletivo as medidas $\mu_{j}$. Devemos, portanto, nos preocupar primeiramente com o estudo individual de cada $\mu_{j}$.

Para tanto, adaptamos e aprimoramos alguns resultados obtidos por Zlatoš em [Z]. Podemos destacar principalmente o Teorema 3.2.5, que demonstra a distribuição uniforme dos ângulos de Prüfer (vide as Seções 3.1 e 3.2) no intervalo $[0, \pi$ ) (com exceção do conjunto $\mathbb{Q} \pi$, de medida de Lebesgue nula $)^{\dagger}$. Essa técnica, além de mais simples e direta na determinação do comportamento assintótico exato da seqüência dos raios de Prüfer (uma vez que a uniformidade na distribuição da seqüência dos ângulos de Prüfer garante a validade do Teorema ergódico 3.1.4), produz um resultado mais geral do que Zlatoš, que deve excluir do espectro toda "energia" $\lambda_{j}$ tal que $\operatorname{dist}(q \lambda, \mathbb{Z}) \leq \frac{1}{q^{2}}$ para $q$ suficientemente grande a fim de obter um resultado equivalente (vide a Observação 3.2.6).

A Proposição 4.1 .1 (Proposição 3.9 de [CMW1]) é outro desses resultados que aprimoram [Z]. Nela demonstramos a existência, para quase toda "energia" $\lambda_{j} \in[-2,2]$, de uma solução da equação de Schrödinger associada à matriz de Jacobi $J_{\delta, \phi}^{j}$ que cresce polinomialmente; mais ainda, afirmamos que para a mesma energia $\lambda$, existe uma solução subordinada (vide o Apêndice E para uma definição desse conceito) que decresce com a mesma taxa de crescimento da solução lineramente independente obtida anteriormente. O resultado análogo de [Z] (Lema 2.1) envolve estimativas menos precisas.

Tanto a Proposição 4.1.1 quanto o Lema 2.1 de [Z] se baseiam no Teorema F.2.12 (Teorema 8.1 de [LS1]), que além de fornecer uma condição suficiente para a existência de uma solução subordinada, indica o comportamento assintótico desta.

É justamente a adaptação desse resultado e do Teorema F.2.15 (Teorema 1.7 de [LS1]) ao problema de um potencial esparso (Teorema 3.3.1) que nos permite obter o Teorema 3.3.3, ligado à existência de uma transição entre espectros singular-contínuo e puramente pontual; além de garantir a existência da transição (discutida por Marchetti et. al. em [MWGA]), a condição suficiente à existência de espectro puramente pontual fornecida pelo Teorema 3.3.1 nos diz que esta transição é aguda (isto é, não existe uma região de transição entre os tipos espectrais), o que aprimora o Teorema 4.4 de [MWGA].

Como última observação, vale destacar que a demonstração desenvolvida por Zlatoš (Teorema 6.3 de [Z]) com respeito à existência de transição espectral, ao nosso ver, está incompleta, já que parece indicar que medidas com dimensão nula são discretas. Existem alguns exemplos na literatura (vide, por exemplo, [DJLS]) de modelos com espectro puramente singular-contínuo cuja medida espectral possui dimensão Hausdorff nula. Mais ainda, apresentamos na Seção 5.2 um modelo com esparsidade super-geométrica (ou seja, cuja distância entre as barreiras crescem super-geometricamente) que exibe uma transição entre os espectros puramente pontual e singular-contínuo e cuja dimemsão Hausdorff associada à medida espectral é nula (vide a Observação 5.2.6).

Todos esses resultados discutidos acima nos permitiram, através da conhecida teoria

\footnotetext{
${ }^{\dagger}$ A idéia de se estudar a distribuição da seqüência de ângulos de Prüfer é devida a Marchetti et. al. [MWGA], tendo sido aplicada com sucesso para a situação de barreiras distribuídas aleatoriamente por Carvalho et. al. em [CMW1].
} 
de [JL] (vide o Apêndice H), determinar exatamente a dimensão Hausdorff de cada uma das medidas espectrais $\mu_{j}$ (Teorema 4.1.3), em analogia ao resultado obtido por Zlatoš (Teorema 6.3 de $[\mathrm{Z}]$ ).

Necessitamos, no entanto, de uma caracterização coletiva de cada uma das medidas espectrais $\mu_{j}$, o que exige uma adaptação da teoria de Jitomirskaya e Last. Os resultados de nossos esforços nessa direção se encontram na Seção 4.2, particularmente no Teorema 4.2.1 (que generaliza o Teorema 1.2 de [JL]; este nos permite definir continuidade ou singularidade da medida espectral com respeito a medidas Hausdorff a partir da determinação de propriedades locais de escala das soluções da equação de Schrödinger) e nos subseqüentes Corolários (4.2.3) (relacionado ao Corolário 4.1 de [JL]) e (4.2.5) (extensão do Corolário 4.5 de [JL]).

Por fim, tendo em mãos essas novas ferramentas, fomos capazes de determinar com exatidão a dimensão Hausdorff da medida espectral matricial associada ao operador (2.4.1) (na realidade, obtemos a dimensão Hausdorff da medida de mistura restrita a cada um dos espaços invariantes de multiplicidade constante; vide o Lema 2.3.8 e o Teorema 4.2.6). O resultado é em princípio surpreendente, já que a dimensão Hausdorff local (tomada em um ponto $\lambda$ pertencente ao espectro do operador (2.4.1)) dessa medida é a menor entre as dimensões de cada uma das medidas $\mu_{j}$ (que contem o ponto $\lambda$ em seu suporte), o que resulta em um comportamento bastante singular para a medida espectral matricial.

Até onde sabemos, este é o primeiro trabalho em que se obtém explicitamente a dimensão Hausdorff da medida espectral de um operador equivalente a uma matriz blocoJacobi. O resultado é por si só bastante interessante e poderia, em princípio, fornecer uma intuição do que ocorre com o modelo no plano $\mathbb{Z}_{+}^{2}$.

\subsection{Roteiro de leitura}

Apresentamos nessa seção um roteiro de leitura da tese, dedicada ao estudo das principais propriedades espectrais da matriz bloco-Jacobi $\mathcal{J}_{\delta, \phi}$ definida no plano $\Lambda$, e tem por objetivo facilitar ao leitor a localização dos principais resultados obtidos.

O Capítulo 2 contém um estudo detalhado da medida espectral matricial $\Omega$, absolutamente contínua com respeito ao seu componente $\Omega_{00}$ (Proposição 2.3.2), bem como a determinação do espectro essencial da matriz bloco-Jacobi $\mathcal{J}_{\delta, \phi}$ (Teorema 2.4.2).

O Capítulo 3 apresenta a demonstração da existência de transição espectral (entre os espectros singular-contínuo e puramente pontual) para cada componente unidimensional do operador $\Pi_{\delta, \phi}$, obtidos a partir do processo de bloco-diagonalização apresentado na Seção 2.1. Esta demonstração envolve o comportamento assintótico das matrizes de transferência associadas às soluções linearmente independentes da equação de Schrödinger (3.4), através dos critérios desenvolvidos por Last e Simon em [LS1], presentes no Apêndice F. Para tanto, adaptamos as variáveis de Prüfer, também definidas no Apêndice F (Seção 3.1); em seguida demonstramos que os ângulos de Prüfer são uniformemente distribuídos 
(Seção 3.2), o que nos permite determinar com exatidão o comportamento assintótico ao qual nos referimos.

No Capítulo 4 obtemos a dimensão Hausdorff da medida espectral matricial $\Omega$. Para tanto, determinamos em primeiro lugar as dimensões de cada componente unidimensional $\mu_{j}$ da medida $\Omega_{00}$ (Teorema 4.1.3) através das técnicas desenvolvidas por Jitomirskaya e Last em [JL] (presentes no Apêndice H). Em seguida, estendemos tais resultados (através do Teorema 4.2.1) para a própria medida de mistura $\Omega_{00}$. O principal resultado é expresso pelo Teorema 4.2.6.

No Capítulo 5 apresentamos alguns outros modelos na faixa $\Lambda$ com barreiras cujas distâncias à origem crescem ora sub, ora super-geometricamente. Verificamos que para a situação com as barreiras de alturas constantes, com esparsidade sub-geométrica, o espectro é puramente pontual (Teorema 5.1.3). Analisamos duas situações com esparsidade super-geométrica: na primeira, com barreiras de alturas constantes, verificamos que o espectro essencial desse operador é puramente singular-contínuo, sendo igual a 1 a dimensão Hausdorff da medida espectral (Teorema 5.2.3); esta é uma situação limítrofe entre os espectros singular-contínuo e absolutamente contínuo. Na segunda, onde a altura das barreiras decai com a distância à origem (vide a relação (5.2.4)), verificamos que o espectro essencial do operador é puramente singular-contínuo, sendo a dimensão Hausdorff da medida espectral igual a zero (Teorema 5.2.7); esta é uma situação limítrofe entre os espectros singular-contínuo e puramente pontual.

No Capítulo 6 apresentamos um resumo e as conclusões dos principais resultados obtidos no trabalho, bem como algumas questões que permanecem sem resposta (principalmente a determinação do comportamento assintótico da transformada de Fourier da medida espectral do operador de diferença finita definido por (1.4)).

Os Apêndices compõem as principais ferramentas que utilizamos ao longo do trabalho, cada qual contendo temas relativamente independentes entre si. Nossa escolha busca enfatizar o trabalho que desenvolvemos, o que não diminui a importância do material presente nos apêndices nem exime o leitor de sua consulta para uma maior compreensão dos resultados apresentados. 


\section{Capítulo 2}

\section{Medida espectral e espectro essencial do operador bloco-Jacobi $\mathcal{J}_{\delta, \phi}$}

Apresentamos nesse capítulo uma caracterização completa da medida espectral matricial e do espectro essencial dos operadores bloco-Jacobi $\mathcal{J}_{\delta, \phi}$ definidos por (1.12), as representações matriciais dos operadores aleatórios auto-adjuntos $\Pi_{P, \phi}$ definidos por (1.6), homogeneamente transversais (Definição 1.2), com $P \in \mathcal{P}_{\delta, \beta}$ (isto é, satisfazendo (1.9)(1.11) $\operatorname{com} \delta \in(0,1)$ fixo).

\subsection{Bloco-diagonalização das matrizes $\mathcal{J}_{\delta, \phi}$}

Por conveniência, alteramos a ordem do produto tensorial em (1.12) através de uma matriz de permutação apropriada, que denotamos por $P$, de tal forma que

$$
I_{L} \otimes J_{\delta, \phi}+A_{L} \otimes I=P\left(J_{\delta, \phi} \otimes I_{L}+I \otimes A_{L}\right) P^{-1} ;
$$

designamos a soma acima também por $\mathcal{J}_{\delta, \phi}$. A soma de Kronecker

$$
\mathcal{J}_{\delta, \phi}=I_{L} \otimes J_{\delta, \phi}+A_{L} \otimes I
$$

é portanto, unitariamente semelhante a (1.12); conseqüentemente, seu espectro permanece inalterado. A permutação na ordem do produto de Kronecker nos permite a blocodiagonalização da matriz $\mathcal{J}_{\delta, \phi}$, comos veremos a seguir, de modo que podemos tratar o problema como uma generalização do caso unidimensional (certamente existem complicações; o problema é, no entanto, essencialmente unidimensional).

A saber, sendo a matriz $A_{L}$ cíclica (já que o operador $\Delta_{0}^{L}$ apresenta condições periódicas de contorno), podemos bloco-diagonalizar a matriz bloco-Jacobi (2.1.1) através da matriz de Fourier $F_{L}$ :

$$
\left(F_{L}^{-1} \otimes I\right)\left(\mathcal{J}_{\delta, \phi}-z I_{L} \otimes I\right)\left(F_{L} \otimes I\right)=\operatorname{diag}\left\{\left(J_{\delta, \phi}-z_{j} I\right)\right\}_{j=0}^{L-1},
$$


onde $z_{j}=z-2 \cos (2 \pi j / L), j=0, \ldots, L-1$, e $F_{L}:=\left[v_{1} v_{2} \cdots v_{L}\right]$ é a matriz construída a partir dos autovetores $v_{k}=\left(1, \xi^{k}, \ldots, \xi^{(L-1) k}\right) / \sqrt{L}, \xi=\exp \{2 \pi i / L\}$ da matriz de deslocamento $S:\left(x_{0}, \ldots, x_{L-1}\right) \longrightarrow\left(x_{1}, \ldots, x_{L-1}, x_{0}\right)$ em suas colunas.

Observação 2.1.1 Os resultados apresentados nesse trabalho não se restringem ao operador $\Pi_{\delta, \phi}$. Podemos estender nossas técnicas a qualquer perturbação bloco-diagonalizável, isto é, que possa ser decomposta em seus componentes unidimensionais por meio de uma transformação de similaridade. Os resultados também são válidos caso o parâmetro $\phi$ associado à condição de contorno (1.7) seja diferente para cada m.

\subsection{Funções de Green e $M$ de Weyl Titchmarsh ma- triciais e a medida espectral}

Assim como no caso unidimensional, as propriedades espectrais do operador $\prod_{\delta, \phi}$ (ou da matriz bloco-Jacobi $\mathcal{J}_{\delta, \phi}$, sua representação matricial; vide a Observação D.1.1), bem como de qualquer outro operador auto-adjunto definido em $l^{2}\left(\Lambda, \mathbb{C}^{L}\right)$, estão diretamente relacionadas ao comportamento da função de Green matricial $\mathcal{G}(z)$ para $z=\lambda+i \varepsilon$. Explicitamente,

$$
\begin{aligned}
\mathcal{G}_{m n}(i, j ; z) & :=\left\langle\left(\mathcal{J}_{\delta, \phi}-z I \otimes I_{L}\right)^{-1} e_{(i, m)}, e_{(j, n)}\right\rangle \\
& =\left\langle P^{-1}\left(\mathcal{J}_{\delta, \phi}-z I_{L} \otimes I\right)^{-1} P e_{(i, m)}, e_{(j, n)}\right\rangle \\
& =\left\langle\left(F_{L} \otimes I\right) \operatorname{diag}\left\{\left(J_{\delta, \phi}-z_{k} I\right)^{-1}\right\}_{k=0}^{L-1}\left(F_{L}^{-1} \otimes I\right) e_{m}^{L} \otimes e_{i}, e_{n}^{L} \otimes e_{j}\right\rangle \\
& =\left\langle\operatorname{diag}\left\{\left(J_{\delta, \phi}-z_{k} I\right)^{-1}\right\}_{k=0}^{L-1} F_{L}^{-1} e_{m}^{L} \otimes e_{i}, F_{L}^{\dagger} e_{n}^{L} \otimes e_{j}\right\rangle \\
& =\left\langle\operatorname{diag}\left\{\left(J_{\delta, \phi}-z_{k} I\right)^{-1}\right\}_{k=0}^{L-1} \bar{v}_{m} \otimes e_{i}, \bar{v}_{n} \otimes e_{j}\right\rangle \\
& =\frac{1}{L}\left(\begin{array}{c}
\bar{\xi}^{m}\left(J_{\delta, \phi}-z_{0} I\right)^{-1} e_{i} \\
\vdots \\
\bar{\xi}^{(L-1) m}\left(J_{\delta, \phi}-z_{L-1} I\right)^{-1} e_{i}
\end{array}\right)^{T}\left(\begin{array}{c}
e_{j} \\
\xi^{n} e_{j} \\
\vdots \\
\xi^{(L-1) n} e_{j}
\end{array}\right) \\
& =\frac{1}{L} \sum_{l=0}^{L-1} \xi^{-l m}\left(\left(J_{\delta, \phi}-z_{l} I\right)^{-1} e_{i}, e_{j}\right)_{1} \xi^{l n} \\
& =\frac{1}{L} \sum_{l=0}^{L-1} \xi^{-l m} G\left(i, j ; z_{l}\right) \xi^{l n},
\end{aligned}
$$

onde a seqüência $\left\{e_{i}\right\}_{i \geq 0}$ representa a base canônica de $l^{2}\left(\mathbb{Z}_{+}, \mathbb{C}\right.$ ) (vide a Observação D.2.2) e a seqüência $\left\{e_{i}^{L}\right\}_{i=0}^{L-1}$ a base canônica do espaço $\mathbb{C}^{L}$; usamos na terceira identidade o fato da matriz de permutação $P$ inverter a ordem do produto direto $e_{(i, m)}=e_{i} \otimes e_{m}^{L}$ e a identidade

$$
\left(F_{L}^{-1} \otimes I\right)\left(\mathcal{J}_{\delta, \phi}-z I_{L} \otimes I\right)^{-1}\left(F_{L} \otimes I\right)=\operatorname{diag}\left\{\left(J_{\delta, \phi}-z_{j} I\right)^{-1}\right\}_{j=0}^{L-1}
$$


(decorrente do fato conhecido de que a matriz inversa à soma de Kronecker $A \otimes I+I \otimes B$ é também uma soma de Kronecker, da forma $A^{-1} \otimes I+I \otimes B^{-1}$; vide [L]); na quinta identidade usamos a definição dos vetores coluna $v_{k}, k=1, \ldots, L$, e a identidade $F_{L}^{-1}=$ $F_{L}^{\dagger}=F_{L}^{*}$; a sexta identidade segue da definição do produto interno sesquilinear $(\cdot, \cdot)_{2}$; a última identidade envolve a definição da função de Green associada à matriz de Jacobi $J_{\delta, \phi}$, calculada no ponto $z_{l} \in \mathbb{C}_{+}, l \in\{0, \ldots, L-1\}$. A função de Green $G\left(i, j ; z_{l}\right)$ é expressa pela fórmula geral (D.2.2), onde $\chi\left(z_{j}\right):=-u^{N}\left(z_{j}\right)+m_{j}(z) u^{D}\left(z_{j}\right), u^{D}\left(z_{j}\right)$ e $u^{N}\left(z_{j}\right)$ as soluções da equação de Schrödinger

$$
a_{n} u_{n+1}+a_{n-1} u_{n-1}+\left(b_{n}-z_{l}\right) u_{n}=0
$$

que satisfazem as condições iniciais ortogonais (D.1.5); $m_{l}(z)$ representa a função de Weyl-Titchmarsh ligada à solução $l^{2}\left(\mathbb{Z}_{+}, \mathbb{C}\right)$ de $(2.2 .2)$.

Observação 2.2.1 Omitimos o índice $\infty$ da função $m_{l}$ a fim de não sobrecarregar a notação.

Fica claro de (2.2.1) e da definição da matriz de Fourier $F_{L}$ que

$$
\mathcal{G}(i, j ; z)=F_{L} \operatorname{diag}\left\{G\left(i, j ; z_{l}\right)\right\}_{l=0}^{L-1} F_{L}^{-1},
$$

$i, j \in \mathbb{Z}_{+}$. Em particular, segue mais uma vez da relação (D.2.2), das identidades (2.2.3) e de (D.2.1), a igualdade

$$
\mathcal{G}(z):=\mathcal{G}(0,0 ; z)=F_{L} \operatorname{diag}\left\{G\left(0,0 ; z_{l}\right)\right\}_{l=0}^{L-1} F_{L}^{-1}=F_{L}\left\{m_{l}(z)\right\}_{l=0}^{L-1} F_{L}^{-1},
$$

resultado este que nos permite definir a matriz $M$ de Weyl-Tithmarsh como

$$
M_{\infty}(z):=F_{L}\left\{m_{l}(z)\right\}_{l=0}^{L-1} F_{L}^{-1}
$$

Se $\left\{P_{\Gamma}\right\}$ é uma família de projetores espectrais com respeito ao subconjunto de Borel $\Gamma$ associada à matriz de Jacobi $J_{\delta, \phi}$, de modo que $J_{\delta, \phi}=\int \lambda d P_{\lambda}$ (vide o Teorema espectral B.2.6), temos de (D.3.1) e (2.2.1)

$$
\mathcal{G}_{m n}(z)=\int_{-\infty}^{\infty} \frac{d \rho_{m, n}(\lambda)}{\lambda-z}=\frac{1}{L} \sum_{l=0}^{L-1} \xi^{l(n-m)} \int_{-\infty}^{\infty} \frac{d \rho_{\psi}(\lambda)}{\lambda-z_{l}},
$$

onde $\rho_{\psi}=\left(P_{\lambda} \psi, \psi\right)$ é a medida espectral de $J_{\delta, \phi}$, com $\psi$ um vetor cíclico em $l^{2}\left(\mathbb{Z}_{+}, \mathbb{C}\right)$. Caso efetuemos a mudança de variável $\lambda^{\prime}=\lambda+2 \cos (2 \pi l / L)$, podemos reescrever a função de Green $G\left(z_{l}\right)$ como

$$
G\left(z_{l}\right)=\int_{-\infty}^{\infty} \frac{d \rho_{\psi}\left(\lambda_{l}\right)}{\lambda_{l}-z}
$$

$\operatorname{com} \lambda_{l} \equiv \lambda^{\prime}-2 \cos (2 \pi l / L)$, de tal modo que para todo $\lambda \in \mathbb{R}$,

$$
d \rho_{m n}(\lambda)=\frac{1}{L} \sum_{l=0}^{L-1} \xi^{l(n-m)} d \rho_{\psi}\left(\lambda_{l}\right)
$$


A medida matricial de Borel-Stietjes $\Omega_{\psi}$ gerada por $\left(d \rho_{m, n}\right)_{m, n=0}^{L-1}$ (isto é, $\Omega_{m n}=$ $\left.\int_{A} d \rho_{m n}(\lambda)\right)$ é a chamada medida espectral matricial associada ao operador ou matriz bloco-Jacobi $\mathcal{J}_{\delta, \phi}$. Sendo $\psi$ um vetor cíclico de $J_{\delta, \phi}$ em $l^{2}\left(\mathbb{Z}_{+}, \mathbb{C}\right)^{\dagger}$, temos que as propriedades espectrais de $\mathcal{J}_{\delta, \phi}$ são completamente descritas pela medida matricial $\Omega_{\psi}$ (vide o Apêndice B para os detalhes).

Observação 2.2.2 Assim como com o caso unidimensional, omitimos a dependência do vetor $\psi$ na medida $\Omega$, sendo esta subentendida. Fazemo-lo assim a fim de não carregar a notação.

Segue diretamente das definições (2.2.4), (2.2.5), e da relação (2.2.6) que

$$
M_{\infty}(z)=\int_{-\infty}^{\infty} \frac{d \Omega(\lambda)}{\lambda-z}
$$

ou seja, que a função matricial $M_{\infty}(z)$ é a transformada de Borel da medida espectral matricial $\Omega$ com respeito a $z \in \mathbb{C}$, em analogia a (D.4.1).

Observação 2.2.3 $\mathrm{Na}$ realidade, poderíamos definir a matriz $M$ de tal forma que a relação (2.2.7) fosse satisfeita.

A fórmula de inversão de Borel-Stieltjes para $\Omega$ é dada, em analogia a (D.4.2), por (vide Teorema 5.4 de $[\mathrm{GT}]$ )

$$
\Omega\left(\left(\lambda_{1}, \lambda_{2}\right)\right)+\frac{1}{2}\left[\Omega\left(\left\{\lambda_{1}\right\}\right)+\Omega\left(\left\{\lambda_{2}\right\}\right)\right]=\lim _{\varepsilon \downarrow 0} \frac{1}{\pi} \int_{\lambda_{1}}^{\lambda_{2}} \Im M_{\infty}(\lambda+i \varepsilon) d \lambda,
$$

onde $\lambda_{1}<\lambda<\lambda_{2}$.

\subsection{Caracterização da medida espectral matricial $\Omega$}

Busquemos descrever em detalhes as principais características da medida espectral matricial $\Omega$. Notando inicialmente que a matriz $M_{\infty}(z)$ definida por (2.2.4) é uma função Herglotz matricial (vide Seção 5 de [GT] para uma discussão das propriedades gerais dessas funções), concluímos que a matriz $\left(\Omega_{i j}(B)\right)_{0 \leq i, j \leq L-1}=\left(d \rho_{i j}(B)\right)_{0 \leq i, j \leq L-1}$ é não-negativa definida para qualquer conjunto de Borel $B \subseteq \mathbb{R}$.

Observação 2.3.1 É possivel mostrar diretamente que a medida espectral matricial $\Omega(B)$ definida por (2.2.5) é não-negativa definida; de fato, segue de (2.2.6) a identidade

$$
\Omega(B)=F_{L} \operatorname{diag}\left\{\mu\left(B_{l}\right)\right\}_{l=0}^{L-1} F_{L}^{-1},
$$

válida para qualquer conjunto de Borel $B \subseteq \mathbb{R}$, com $B_{k}=B-2 \cos (2 \pi k / L)$ o "deslocamento" de B pelo fator $2 \cos (2 \pi k / L)$. Como a matriz diag $\left\{\mu\left(B_{l}\right)\right\}_{l=0}^{L-1}$ é não-negativa

\footnotetext{
${ }^{\dagger}$ ou seja, o conjunto $\left\{\left(J_{\delta, \phi}\right)^{k} \psi\right\}_{k=0}^{\infty}$ é denso no espaço $l^{2}\left(\mathbb{Z}_{+}, \mathbb{C}\right)$
} 
definida (já que $\mu_{\psi}\left(B_{l}\right)=\int_{B_{l}} d\left\langle P_{\lambda} \psi, \psi\right\rangle, l=\{0, \ldots, L-1\}$, são funções não-decrescentes, contínuas à direita e satisfazem os limites $\lim _{\lambda \rightarrow-\infty} \mu(\lambda)=0$ e $\left.\lim _{\lambda \rightarrow \infty} \mu(\lambda)=1\right)$ e $F_{L}^{-1}$ é não-singular, segue da Observação 7.1 .6 de $[H J]$ que $\left(F_{L}^{-1}\right)^{*} \operatorname{diag}\left\{\mu\left(B_{l}\right)\right\}_{l=0}^{L-1} F_{L}^{-1}=\Omega(B)$ é não-negativa definida.

Um resultado fundamental na caracterização da medida matricial $\Omega$ é exibido na seguinte

Proposição 2.3.2 A medida espectral matricial $\Omega$ é absolutamente contínua com respeito à medida $\Omega_{00}$, isto é, $\Omega(B)=0$ para todo subconjunto de Borel $B \subseteq \mathbb{R}$ tal que $\Omega_{00}(B)=0$.

Observação 2.3.3 É simples notar da relação (2.2.6) que $\Omega_{00}=\Omega_{m m}$ para todo $m \in$ $\{0, \ldots, L-1\}$, isto é, que todos os elementos diagonais da matriz $\Omega$ são idênticos. Poderíamos, portanto, exprimir os resultados da Proposição 2.3.2 em termos de qualquer um desses elementos.

Demonstração. Fica claro a partir de (2.2.6) que para todos $m, n=0, \ldots, L-1$, e para todo subconjunto de Borel $B \subseteq \mathbb{R}$,

$$
\Omega_{m n}(B) \leq\left|\Omega_{m n}(B)\right| \leq \frac{1}{L}\left|\sum_{l=0}^{L-1} \xi^{l(n-m)} \int_{B_{l}} d \rho\left(B_{l}\right)\right| \leq \frac{1}{L} \sum_{l=0}^{L-1} \mu\left(B_{l}\right)=\Omega_{00}(B),
$$

$B_{l}$ os conjuntos de Borel definidos como na Observação 2.3.1. Isso encerra a demonstração.

A Proposição 2.3.2 nos indica que as principais características da medida espectral matricial $\Omega$, tais como seu espectro essencial e sua continuidade ou singularidade com respeito às medidas de Lebesgue $\kappa$ e Hausdorff $h^{\alpha}$, são inteiramente determinadas pela medida $\Omega_{00}$. No entanto, a questão da multiplicidade espectral (ligada à existência de múltiplos vetores cíclicos) não depende apenas da caracterização de $\Omega_{00}$ e deve ser tratada com as ferramentas apropriadas.

Observação 2.3.4 Os resultados seguintes são uma generalização, para matrizes $L \times L$, $L \geq 2$, dos resultados do Apêndice $B$ de [T] para $L=2$.

Para tanto, apresentemos a seguinte

Definição 2.3.5 A forma sesquilinear

$$
\langle\mathbf{f}, \mathbf{g}\rangle_{\Omega}=\int_{\mathbb{R}}(d \Omega(\lambda) \mathbf{f}, \mathbf{g})=\int_{\mathbb{R}} \sum_{i, j=0}^{L-1} f_{i}(\lambda) g_{j}^{*}(\lambda) d \rho_{i j}(\lambda)
$$

onde $\mathbf{f}=\left(f_{0}, \ldots, f_{L-1}\right), f_{i}, g_{i} \in L^{2}\left(\mathbb{R}, \mathbb{C}, d \rho_{00}\right)$, é dita não-negativa definida caso a desigualdade $\|\mathbf{f}\|_{\Omega}^{2}=\langle\mathbf{f}, \mathbf{f}\rangle_{\Omega} \geq 0$ seja satisfeita nesse espaço. 
Consideremos a forma sesquilinear definida por (2.3.2). Suponha que f seja uma função simples, ou seja, que $\mathbf{f}(\lambda)=\sum_{l=0}^{L-1} \chi_{B_{l}}(\lambda)\left(f_{l, 0}, \ldots, f_{l, L-1}\right)$, onde $\left(f_{l, 0}, \ldots, f_{l, L-1}\right) \in \mathbb{C}^{L}$ e $B_{l}$ são conjuntos de Borel disjuntos. Para esta classe de funções, (2.3.2) é uma forma sesquilinear não-negativa definida. Se f é tal que $f_{i} \in L^{2}\left(\mathbb{R}, \mathbb{C}, d \rho_{00}\right)$, podemos aproximála por funções simples de tal modo que $\|\mathbf{f}\|_{\Omega} \geq 0$. Temos, como conseqüência, um espaço de Hilbert separável $L^{2}\left(\mathbb{R}, \mathbb{C}^{L}, d \Omega\right)$, com produto escalar definido por (2.3.2).

Em seguida, como as matrizes de Jacobi $J_{\delta, \phi}$ em consideração satisfazem o caso pontolimite (já que $\sum_{n \geq 0}\left|a_{n}\right|^{-1}=\sum_{n \geq 0}^{\infty} p_{n}^{-1}=\sum_{n \in \mathcal{A}}(1-\delta)^{-1}+\sum_{n \notin \mathcal{A}} 1=\infty$; vide Proposição D.1.2), existe uma transformação unitária $\tilde{U}: l^{2}\left(\mathbb{Z}_{+}, \mathbb{C}\right) \longrightarrow L^{2}\left(\mathbb{R}, \mathbb{C}, d \rho_{00}\right)$, tal que $\Pi_{\delta, \phi}=$ $\left(\tilde{U}^{-1} \otimes I_{L}\right) \tilde{\Pi}_{\delta, \phi}\left(\tilde{U} \otimes I_{L}\right)$, onde $\tilde{\Pi}_{\delta, \phi}: L^{2}\left(\mathbb{R}, \mathbb{C}^{L}, d \Omega\right) \longrightarrow L^{2}\left(\mathbb{R}, \mathbb{C}^{L}, d \Omega\right)$ é o operador de multiplicação por $\lambda$ no espaço $L^{2}\left(\mathbb{R}, \mathbb{C}^{L}, d \Omega\right.$ ) (vide, por exemplo, o Teorema 2.12 de $[\mathrm{T}]$ para a demonstração).

Temos o importantíssimo

Lema 2.3.6 (Lema 2.7 de [CMW]) $O$ conjunto $\sigma\left(\Omega_{00}\right):=\left\{\lambda \in \mathbb{R}: \Omega_{00}((\lambda-\varepsilon, \lambda+\right.$ $\varepsilon))>0$ para qualquer $\varepsilon>0\}$ é precisamente o espectro $\sigma\left(\tilde{\Pi}_{\delta, \phi}\right)$ do operador de multiplicação $\tilde{\Pi}_{\delta, \phi} \mathbf{f}(\lambda)=\lambda \mathbf{f}(\lambda)$, com domínio $\mathcal{D}\left(\tilde{\Pi}_{\delta, \phi}\right)=\left\{\mathbf{f} \in L^{2}\left(\mathbb{R}, \mathbb{C}^{L}, d \Omega\right) \mid \lambda \mathbf{f}(\lambda) \in\right.$ $\left.L^{2}\left(\mathbb{R}, \mathbb{C}^{L}, d \Omega\right)\right\}$.

Observação 2.3.7 O Lema 2.3.6 é uma extensão do Lema B.5 de [T] para operadores multidimensionais. Preferimos apresentar sua demonstração nesse momento dada sua importância.

Demonstração. Se $\lambda \in \sigma\left(\Omega_{00}\right)$, então a seqüência $\mathbf{f}_{n}=\frac{1}{\sqrt{L}} \Omega_{00}\left(\left(\lambda-\frac{1}{n}, \lambda+\frac{1}{n}\right)\right)^{-1 / 2}$ $\chi_{\left(\lambda-\frac{1}{n}, \lambda+\frac{1}{n}\right)}(1,1, \ldots, 1)$ satisfaz $\left\|\mathbf{f}_{n}\right\|=1$ e $\left\|\left(\tilde{\Pi}_{\delta, \phi}-\lambda\right) \mathbf{f}_{n}\right\| \rightarrow 0$, e portanto $\lambda \in \sigma\left(\tilde{\Pi}_{\delta, \phi}\right)$, pelo critério de Weyl (Teorema B.2.13).

Suponhamos agora que $\zeta \notin \sigma\left(\Omega_{00}\right)$. Segue da Proposição 2.3.2 que $\zeta \notin \sigma(\Omega)$, e que portanto o operador $\left(\tilde{\Pi}_{\delta, \phi}-\zeta\right)^{-1} \mathbf{f}(\lambda)=(\lambda-\zeta)^{-1} \mathbf{f}(\lambda)$ existe e é limitado; logo, $\zeta \notin \sigma\left(\tilde{\Pi}_{\delta, \phi}\right)$, pela Definição A.1.1.

O Lema 2.3.6 nos mostra que o espectro do operador $\Pi_{\delta, \phi}$ (idêntico ao espectro do operador de multiplicação $\tilde{\Pi}_{\delta, \phi}$ no espaço $L^{2}\left(\mathbb{R}, \mathbb{C}^{L}, d \Omega\right)$, uma vez que estes são unitariamente equivalentes; o espectro é invariante por transformações unitárias) é justamente o conjunto $\sigma\left(\Omega_{00}\right)$, o que mais uma vez evidencia a relevância da medida $\Omega_{00}$.

Mais ainda, podemos usá-la na diagonalização da medida espectral matricial $\Omega$. Como suas componentes $\Omega_{i j}$ são absolutamente contínuas com respeito a $\Omega_{00}$ (vide a Proposição 2.3.2), existe uma matriz simétrica e não-negativa $Y(\lambda)$ tal que, pelo Teorema de RadonNikodym,

$$
d \Omega(\lambda)=Y(\lambda) d \rho_{00}(\lambda)
$$


e cujos elementos são dados por

$$
Y_{i j}(\lambda)=\frac{d \rho_{i j}(\lambda)}{d \rho_{00}(\lambda)}=\lim _{\epsilon \downarrow 0} \frac{\Im\left(\left(M_{\infty}\right)_{i j}(\lambda+i \epsilon)\right)}{\Im\left(\left(M_{\infty}\right)_{00}(\lambda+i \epsilon)\right)}
$$

(usamos a fórmula de inversão de Stieltjes (2.2.8) na obtenção da última identidade). Temos explicitamente da definição (2.2.4) da matriz $M_{\infty}(z)$

$$
\begin{aligned}
\left(M_{\infty}\right)_{m n}(z) & =\frac{1}{L} \sum_{j, k=0}^{L-1} \bar{\xi}^{m j}\left(\operatorname{diag}\left\{m_{l}(z)\right\}_{l=0}^{L-1}\right)_{j k} \xi^{k n}=\frac{1}{L} \sum_{j, k=0}^{L-1} m_{l}(z) e^{2 \pi i(n-m) j / L} \\
& =\frac{1}{L} \sum_{j=0}^{L-1}\left[\mathcal{R} m_{j}(z) \cos \left(\frac{2 \pi(n-m) j}{L}\right)-\mathcal{I} m_{j}(z) \sin \left(\frac{2 \pi(n-m) j}{L}\right)\right] \\
& +\frac{i}{L} \sum_{j=0}^{L-1}\left[\mathcal{R} m_{j}(z) \sin \left(\frac{2 \pi(n-m) j}{L}\right)+\mathcal{I} m_{j}(z) \cos \left(\frac{2 \pi(n-m) j}{L}\right)\right] .
\end{aligned}
$$

Se levarmos em conta que $z_{j}=z_{L-j}$ (uma vez que $\cos (2 \pi j / L)=\cos (2 \pi(L-j) / L)$ ) e que $\sin (2 \pi j / L)=-\sin (2 \pi(L-j) / L)$, temos da relação acima e do Lema D.3.5 a identidade

$$
\left(\Im M_{\infty}\right)_{m n}(z)=\frac{1}{L} \sum_{j=0}^{L-1} \mathcal{I} m_{j}(z) \cos \left(\frac{2 \pi(n-m)}{L} j\right) .
$$

Fica claro de (2.3.4) que $Y(\lambda)$ também é uma matriz cíclica, e que portanto, pode ser diagonalizada pela matriz de Fourier $F_{L}$ :

$$
Y(\lambda)=F_{L} \operatorname{diag}\left\{y_{j}(\lambda)\right\}_{j=0}^{L-1} F_{L}^{-1}
$$

$\operatorname{com} y_{j}(\lambda)=\lim _{\varepsilon \downarrow 0} L \Im m_{j}(\lambda+i \varepsilon) / \sum_{n=0}^{L-1} \Im m_{n}(\lambda+i \varepsilon), \lambda_{j}=\lambda-2 \cos (2 \pi j / L)$, os autovalores de $Y(\lambda)$. Este último resultado segue de

$$
\begin{aligned}
\left(F_{L}^{-1} Y(\lambda) F_{L}\right)_{m n} & =\frac{1}{L} \sum_{j, k=0}^{L-1} \xi^{m j} Y_{j k}(\lambda) \bar{\xi}^{k n} \\
& =\frac{1}{2 L} \lim _{\varepsilon \downarrow 0} \frac{\sum_{j, k=0}^{L-1} \xi^{m j-k n} \sum_{l=0}^{L-1} \Im m_{l}\left(\lambda_{+} i \varepsilon\right)\left(\xi^{(j-k) l}+\xi^{-(j-k) l}\right)}{\sum_{l=0}^{L-1} \Im m_{l}(\lambda+i \varepsilon)} \\
& =\frac{1}{2 L} \lim _{\varepsilon \downarrow 0} \frac{\sum_{l=0}^{L-1} \Im m_{l}(\lambda+i \varepsilon) \sum_{j, k=0}^{L-1}\left(\xi^{(m-l) k-(n-l) j}+\xi^{(m+l) k-(n+l) j}\right)}{\sum_{l=0}^{L-1} \Im m_{l}(\lambda+i \varepsilon)} \\
& =\frac{L}{2} \lim _{\varepsilon \downarrow 0} \frac{\sum_{l=0}^{L-1} \Im m_{l}(\lambda+i \varepsilon)\left(\delta_{n, l} \delta_{m, l}+\delta_{L-n, l} \delta_{L-m, l}\right)}{\sum_{l=0}^{L-1} \Im m_{l}(\lambda+i \varepsilon)} \\
& =L \lim _{\varepsilon \downarrow 0} \frac{\Im m_{n}(\lambda+i \varepsilon) \delta_{m, n}}{\sum_{l=0}^{L-1} \Im m_{l}(\lambda+i \varepsilon)}
\end{aligned}
$$


a segunda identidade resulta de (2.3.3), (2.3.4) e de $2 \cos (2 \pi(j-k) l / L)=\left(\xi^{(j-k) l}+\right.$ $\left.\xi^{-(j-k) l}\right) / 2$; a quarta identidade é obtida a partir das relações $\sum_{i=0}^{L-1} \xi^{(n-l) i}=L \delta_{n, l}$ e $\sum_{i=0}^{L-1} \xi^{(n+l) i}=L \delta_{L-n, l}$.

A matriz de Fourier $F_{L}$ age como um operador unitário

$$
L^{2}\left(\mathbb{R}, \mathbb{C}^{L}, d \Omega\right) \rightarrow \bigoplus_{j=0}^{L-1} L^{2}\left(\mathbb{R}, \mathbb{C}, y_{j} d \rho_{00}\right), \quad \mathbf{f}(\lambda) \mapsto F_{L} \mathbf{f}(\lambda)
$$

que torna $\tilde{\Pi}_{\delta, \phi}$ invariante (com respeito ao produto interno definido em $L^{2}\left(\mathbb{R}, \mathbb{C}^{L}, d \Omega\right)$ ). Esse fato é fundamental na investigação da multiplicidade espectral de $\tilde{\Pi}_{\delta, \phi}$ (e de $\Pi_{\delta, \phi}$, conseqüentemente).

Lema 2.3.8 (Lema 2.8 de [CMW]) Defina os conjuntos

$$
B^{(k)}=\left\{\lambda \in \sigma\left(\Omega_{00}\right): k \text { autovalores de } Y(\lambda) \text { são distintos de zero }\right\},
$$

$k=1, \ldots, L . \quad$ Então, $\tilde{\Pi}_{\delta, \phi}=\bigoplus_{k=1}^{L} \chi_{B^{(k)}} \tilde{\Pi}_{\delta, \phi}$, e a multiplicidade espectral do operador $\chi_{B^{(k)}} \tilde{\Pi}_{\delta, \phi} e^{k} k$.

Observação 2.3.9 1. O Lema 2.3.8 é a extensão do Lema B.14 de [T] para operadores com $L \geq 2$.

2. O operador $\chi_{B^{(k)}} \tilde{\Pi}_{\delta, \phi}$ é a restrição do operador $\tilde{\Pi}_{\delta, \phi}$ ao subespaço $B^{(k)} k$-dimensional (existem nesse subespaço $k$ vetores cíclicos, linearmente independentes, de $\tilde{\Pi}_{\delta, \phi}$ ).

Demonstração. Não é difícil notar que o operador $\chi_{B^{(1)}} \tilde{\Pi}_{\delta, \phi}$ é unitariamente equivalente ao operador de multiplicação por $\lambda$ em $L^{2}\left(\mathbb{R}, \chi_{B^{(1)}} d \rho_{00}\right)$. Uma vez que $y_{j_{l}} \chi_{B^{(k)}} d \rho_{00}$ ( $y_{j_{l}}$ um dos $k$ autovalores de $Y$ diferentes de zero) e $\chi_{B^{(k)}} d \rho_{00}$ são mutuamente absolutamente contínuos, $l=1, \ldots, k, \chi_{B^{(k)}} \tilde{\Pi}_{\delta, \phi}$ é unitariamente equivalente ao operador de multiplicação por $\lambda$ no espaço de Hilbert $L^{2}\left(\mathbb{R}, I_{k} \chi_{B^{(k)}} d \rho_{00}\right)$ para todo $k>1$, onde $I_{k}$ representa a matriz identidade $k \times k$. Isso encerra a demonstração.

Por fim, temos a interessante

Proposição 2.3.10 Sejam $\mu_{\psi}$ e $\nu_{\xi}$ as medidas espectrais associadas à matriz de Jacobi $J_{\delta, \phi}\left(\right.$ definida em $l^{2}\left(\mathbb{Z}_{+}, \mathbb{C}\right)$, com produto interno $\left.(\cdot, \cdot)_{1}\right)$ e $A_{L}$ (definida em $\mathbb{C}^{L}$, com produto interno $\left.(\cdot, \cdot)_{2}\right)$, respectivamente, geradas pelas funções $\rho_{\psi}(x)=\left(E_{x} \psi, \psi\right)_{1}, \psi \in l^{2}\left(\mathbb{Z}_{+}, \mathbb{C}\right)$ $e \gamma_{e_{0}^{L}}(y)=\left(F_{y} \xi, \xi\right)_{2}, e_{0}^{L} \in \mathbb{C}^{L}\left(\left\{E_{\Gamma}\right\}\right.$ e $\left\{F_{\Lambda}\right\}$ são as m.v.p's associadas à matriz de Jacobi $J_{\delta, \phi}$ e à matriz $A_{L}$, respectivamente). Se $\Omega_{00}$ denota o elemento 00 da medida espectral matricial relacionada à matriz bloco-Jacobi $\mathcal{J}_{\delta, \phi}$, então

$$
\Omega_{00}(B)=\left(\mu_{\psi} * \nu_{e_{0}^{L}}\right)(B)
$$

para qualquer subconjunto de Borel $B \subseteq \mathbb{R}$, onde definimos o produto de convolução

$$
\left(\mu_{\psi} * \nu_{e_{0}^{L}}\right)(B)=\int_{-\infty}^{\infty} \mu_{\psi}(B-y) d \gamma_{e_{0}^{L}}(y)=\int_{-\infty}^{\infty} \nu_{e_{0}^{L}}(B-x) d \rho_{\psi}(x)
$$


Demonstração. Sabemos do Teorema espectral B.1.5 que o elemento $\Omega_{00}$ da medida espectral matricial associada à matriz bloco-Jacobi $\mathcal{J}_{\delta, \phi}$ é univocamente determinado por

$$
\left\langle f\left(\mathcal{J}_{\delta, \phi}\right) \Psi, \Psi\right\rangle=\int_{-\infty}^{\infty} f(x) d \Omega_{\Psi}(x)
$$

para qualquer função de Borel $f$. Podemos, dada a estrutura da soma de Kronecker $\mathcal{J}_{\delta, \phi} \otimes I_{L}+I \otimes A_{L}$, decompor o espaço de Hilbert $l^{2}\left(\Lambda, \mathbb{C}^{L}\right)$ no produto tensorial dos espaços $l^{2}\left(\mathbb{Z}_{+}, \mathbb{C}\right)$ e $\mathbb{C}^{L}$; dessa forma, temos $\Psi=\psi \otimes e_{0}^{L}$, com $\psi \in l^{2}\left(\mathbb{Z}_{+}, \mathbb{C}\right)$ e $e_{0}^{L} \in \mathbb{C}^{L}$. Reescrevamos (2.3.9) primeiramente para o caso $f(x)=x^{p}, p \in \mathbb{N}$ :

$$
\begin{aligned}
\int_{-\infty}^{\infty} u^{p} d \Omega_{\Psi}(u) & =\left\langle\mathcal{J}_{\delta, \phi}^{p} \Psi, \Psi\right\rangle=\left\langle\left(\sum_{j=0}^{p}\left(\begin{array}{l}
p \\
j
\end{array}\right) J_{\delta, \phi}^{j} \otimes A_{L}^{p-j}\right) \Psi, \Psi\right\rangle \\
& =\sum_{j=0}^{p}\left(\begin{array}{c}
p \\
j
\end{array}\right)\left(J_{\delta, \phi}^{j} \psi, \psi\right)_{1}\left(A_{L}^{p-j} e_{0}^{L}, e_{0}^{L}\right)_{2} \\
& =\sum_{j=0}^{p}\left(\begin{array}{l}
p \\
j
\end{array}\right) \int_{-\infty}^{\infty} \int_{-\infty}^{\infty} x^{j} y^{p-j} d \rho_{\psi}(x) d \gamma_{e_{0}^{L}}(y) \\
& =\int_{-\infty}^{\infty} \int_{-\infty}^{\infty}(x+y)^{p} d \rho_{\psi}(x) d \gamma_{e_{0}^{L}}(y) .
\end{aligned}
$$

A generalização de (2.3.10) para um polinômio qualquer é imediata. Daí, segue do Teorema de aproximação de Weierstrass (vide [Ru]) a validade de (2.3.10) para qualquer função contínua; a conexão com funções mensuráveis é direta.

Finalmente, segue da mudança de variável $u=x+y$ que

$$
\int_{-\infty}^{\infty} \int_{-\infty}^{\infty} g(x+y) d \rho_{\psi}(x) d \gamma_{e_{0}^{L}}(y)=\int_{-\infty}^{\infty} g(u) d\left(\mu_{\psi} * \nu_{e_{0}^{L}}\right)(u)
$$

para qualquer função Borel mensurável $g$. Escolhamos $g$ como a função indicadora do conjunto de Borel $B$ (isto é $g(x)=\chi_{B}(x)$ ), de modo que, para cada $y, g(x+y)=\chi_{B-y}(x)$. Temos, portanto,

$$
\int_{-\infty}^{\infty} g(x+y) d \rho_{\psi}(x)=\mu_{\psi}(B-y)
$$

cuja substituição no membro direito de (2.3.11) resulta na primeira igualdade de (2.3.8); argumentos análogos nos conduzem à segunda identidade de (2.3.8). Isso encerra a demonstração da proposição.

Observação 2.3.11 Fica claro a partir dos resultados da Proposição 2.3.10 e do exercício 18 da Seção 6.1 de $[C]$ que $\sigma\left(\mathcal{J}_{\delta, \phi}\right)=\overline{\sigma\left(J_{\delta, \phi}\right)+\sigma\left(A_{L}\right)}$, onde "+" designa soma vetorial". O caso particular desse resultado à matriz livre é o conteúdo da Proposição 2.4.1.

\footnotetext{
${ }^{\dagger}$ isto é, $C=A+B$ caso $C \equiv\{x+y: x \in A, y \in B\}$
} 
Vale destacar que a relação (2.3.7) é um caso particular da fórmula

$$
\Omega_{m n}(B)=\left(\mu_{\psi} * \nu_{e_{m}^{L}, e_{n}^{L}}\right)(B)
$$

onde $\nu_{e_{m}^{L}, e_{n}^{L}}(y)=\left(F_{y} e_{m}^{L}, e_{n}^{L}\right)_{2}, m, n=0, \ldots, L-1,\left\{F_{\Gamma}\right\}$ uma família de projetores espectrais associados à matriz $A_{L}$. Explicitamente, no caso $L=2$,

$\left(F_{y} e_{0}^{L}, e_{1}^{L}\right)_{2}=\left(\left(\sum_{y_{j} \leq y} P_{y}\right) e_{0}^{L}, e_{1}^{L}\right)_{2}=\left\{\begin{array}{cc}0 & \text { se } \quad y<-2, \\ \left(\begin{array}{ll}1 & 0\end{array}\right) P_{1}\left(\begin{array}{l}0 \\ 1\end{array}\right)=-1 / 2 & \text { se } \quad-2 \leq y<2, \\ \left(\begin{array}{ll}1 & 0\end{array}\right) P_{2}\left(\begin{array}{l}0 \\ 1\end{array}\right)=0 \quad \text { se } \quad y \geq 2,\end{array}\right.$

onde $P_{1}=\frac{1}{2}\left(\begin{array}{cc}1 & -1 \\ -1 & 1\end{array}\right), P_{2}=\frac{1}{2}\left(\begin{array}{ll}1 & 1 \\ 1 & 1\end{array}\right)$ representam, respectivamente, os projetores ligados aos autovalores $y_{1}=-2, y_{2}=2$;

$$
\left(F_{y} e_{0}^{L}, e_{0}^{L}\right)_{2}=\left(\left(\sum_{y_{j} \leq y} P_{y}\right) e_{0}^{L}, e_{0}^{L}\right)_{2}=\left\{\begin{array}{cl}
\text { se } \quad y<-2, \\
\left(\begin{array}{cc}
1 & 0
\end{array}\right) P_{1}\left(\begin{array}{l}
1 \\
0
\end{array}\right)=1 / 2 & \text { se } \quad-2 \leq y<2 \\
\left(\begin{array}{ll}
1 & 0
\end{array}\right) P_{2}\left(\begin{array}{l}
1 \\
0
\end{array}\right)=1 & \text { se } \quad y \geq 2
\end{array}\right.
$$

(é possivel mostrar que $\left(F_{y} e_{1}^{L}, e_{0}^{L}\right)_{2}=\left(F_{y} e_{0}^{L}, e_{1}^{L}\right)_{2}$ e $\left(F_{y} e_{1}^{L}, e_{1}^{L}\right)_{2}=\left(F_{y} e_{0}^{L}, e_{0}^{L}\right)_{2}$ pelos mesmos argumentos mostrados acima).

O emprego da primeira identidade de (2.3.8) resulta em

$$
\begin{aligned}
&\left(\mu_{\psi} * \nu_{e_{0}^{L}, e_{1}^{L}}\right)(B)=\frac{1}{2} \int_{\sigma\left(J_{\delta, \phi}\right)} \mu_{\psi}(B-y)(\delta(y-2)-\delta(y+2)) d y \\
&=\frac{1}{2}\left(\mu_{\psi}(B-2)-\mu_{\psi}(B+2)\right)=\frac{1}{2}\left(\mu_{\psi}\left(B_{0}\right)-\mu_{\psi}\left(B_{1}\right)\right), \\
&\left(\mu_{\psi} * \nu_{e_{0}^{L}}\right)(B)=\frac{1}{2} \int_{\sigma\left(J_{\delta, \phi}\right)} \mu_{\psi}(B-y)(\delta(y-2)+\delta(y+2)) d y=\frac{1}{2}\left(\mu_{\psi}\left(B_{0}\right)+\mu_{\psi}\left(B_{1}\right)\right),
\end{aligned}
$$

que podem ser diretamente comparadas com (2.3.1).

A Proposição 2.3.10 se torna particularmente útil no estudo do operador $\Pi_{P, Q, \phi, \psi}$, definido por (1.19), já que as técnicas que empregamos no modelo definido na faixa não se aplicam a esse caso. O conhecimento preciso do comportamento assintótico das transformadas de Fourier das medidas $\mu$ e $\nu$ (ligadas às componentes unidimensionais do operador $\left.\Pi_{P, Q, \phi, \psi}\right)$ aliado a (2.3.7) é, como discutido no Capítulo 1 , imperativo na caracterização do espectro de $\Pi_{P, Q, \phi, \psi}$. 


\subsection{Estudo do espectro de $\mathcal{J}_{\delta, \phi}$}

Apresentamos nessa seção os principais resultados referentes ao espectro da matriz blocoJacobi $\mathcal{J}_{\delta, \phi}$, a representação matricial do operador $\Pi_{\delta, \phi}$ definido por (1.6). Caracterizamos em primeiro lugar o espectro da matriz livre $\mathcal{J}_{0}$, e em seguida o espectro essencial da própria matriz $\mathcal{J}_{\delta, \phi}$.

\subsubsection{Características espectrais da matriz livre $\mathcal{J}_{0}$}

A matriz bloco--Jacobi livre $\mathcal{J}_{0}$ associada ao operador livre $\Pi_{0,0}$ é dada pela expressão

$$
\mathcal{J}_{0}=I_{L} \otimes J_{0}+A_{L} \otimes I
$$

com $J_{0}$ a matriz de Jacobi livre

$$
J_{0}=\left(\begin{array}{ccccc}
0 & 1 & 0 & 0 & \cdots \\
1 & 0 & 1 & 0 & \cdots \\
0 & 1 & 0 & 1 & \cdots \\
0 & 0 & 1 & 0 & \cdots \\
\vdots & \vdots & \vdots & \vdots & \ddots
\end{array}\right)
$$

Temos como primeiro resultado a

Proposição 2.4.1 O espectro da matriz bloco-Jacobi livre $\mathcal{J}_{0}$ corresponde ao intervalo fechado

$$
\sigma\left(\mathcal{J}_{0}\right)= \begin{cases}{[-2+2 \cos (\pi(L-1) / L), 4]} & \text { se } L \text { for ímpar } \\ {[-4,4]} & \text { se } L \text { for par }\end{cases}
$$

com $L \geq 2$.

Observação 2.4.2 Como o espectro essencial da matriz de Jacobi $J_{0}, \sigma_{\mathrm{ess}}(J)$, coincide com o próprio espectro $\sigma(J)$ (já que a medida $\Omega_{00}$ é, neste caso, puramente absolutamente contínua; vide a Proposição 2.4.4), segue que

$$
\sigma_{\text {ess }}\left(\mathcal{J}_{0}\right)=\left\{\begin{array}{lll}
{[-2+2 \cos (\pi(L-1) / L), 4]} & \text { se } L \text { for ímpar } \\
{[-4,4]} & \text { se } L \text { for par }
\end{array}\right.
$$

$\operatorname{com} L \geq 2$.

Demonstração. Denotando por $\mu$ a medida espectral associada à matriz de Jacobi livre $J_{0}$, obtemos da Proposição 2.3.10 a identidade

$$
\begin{aligned}
\Omega_{00}((\lambda-\varepsilon, \lambda+\varepsilon)) & =\frac{1}{L} \int_{-\infty}^{\infty} \mu((\lambda-y-\varepsilon, \lambda-y+\varepsilon)) \sum_{l=0}^{L-1} \delta\left(y-y_{j}\right) d y \\
& =\frac{1}{L} \sum_{l=0}^{L-1} \mu\left(\left(\lambda-y_{l}-\varepsilon, \lambda-y_{l}+\varepsilon\right)\right)=\frac{1}{L} \sum_{l=0}^{L-1} \mu_{l}((\lambda-\varepsilon, \lambda+\varepsilon)),
\end{aligned}
$$


onde $\mu_{l}$ representa a medida espectral da matriz de Jacobi $J_{0}^{l}$ associada ao laplaciano livre

$$
\left(\Delta_{0,0}^{l}\right) u_{n}=u_{n+1}+u_{n-1}-y_{l} u_{n}
$$

$y_{l} \equiv \xi^{l}+\bar{\xi}^{l}=2 \cos (2 \pi l / L), l=0, \ldots L-1$.

Sendo o espectro da matriz de Jacobi $J_{0}$ o intervalo fechado $[-2,2]$, segue do resultado acima e da Proposição B.2.7 a identidade

$$
\begin{aligned}
\sigma\left(\mathcal{J}_{0}\right) & =\left\{\lambda \in \mathbb{R}: \Omega_{00}((\lambda-\varepsilon, \lambda+\varepsilon))>0 \text { para todo } \varepsilon>0\right\} \\
& = \begin{cases}{[-2+2 \cos (\pi(L-1) / L), 4]} & \text { se } L \text { for ímpar } \\
{[-4,4]} & \text { se } L \text { for par }\end{cases}
\end{aligned}
$$

com $L \geq 2$. Isso encerra a demonstração.

Observação 2.4.3 Podemos pensar na Proposição 2.4.1 como uma generalização de um conhecido resultado para somas de Kronecker. De fato, se $\left\{\eta_{k}\right\}_{k=1}^{n}$ e $\left\{\lambda_{j}\right\}_{j=1}^{m}$ são os autovalores das matrizes $A$ e $B$, respectivamente, então $\left\{\eta_{k}+\lambda_{j}\right\}_{k, j=1}^{n, m}$ são os autovalores da soma de Kronecker $I_{m} \otimes A+B \otimes I_{n}$ (vide [L] para uma demonstração desse fato). Este é, grosso modo, o resultado apresentado tanto na Proposição 2.3.10 quanto na Proposição 2.4.1.

Como já mencionado, o espectro essencial da matriz de Jacobi $J_{0}$ é puramente absolutamente contínuo. Uma vez que $\mathcal{J}_{0}$ é, em certo sentido, a representação matricial de um operador livre, temos

Proposição 2.4.4 (Proposição 2.1 de [CMW]) O elemento $\Omega_{00}$ da medida espectral matricial $\Omega$, associada à matriz bloco-Jacobi $\mathcal{J}_{0}$, é puramente absolutamente contínuo quando restrito ao conjunto (2.4.3).

Demonstração. O resultado segue trivialmente do fato do produto de convolução de uma medida absolutamente contínua com qualquer medida singular (em particular puramente pontual) ser também uma medida absolutamente contínua (vide Exercício 6 da Seção 6.1 de $[\mathrm{C}]$ ).

Existe uma demonstração alternativa da Proposição 2.4.4 que envolve a obtenção explícita da derivada de Radon-Nikodym $d \rho_{00} / d \kappa$, definida por (D.3.5). Segue da fórmula de inversão de Borel-Stieltjes (2.2.8) e de (2.3.4) que

$$
\begin{aligned}
\lim _{\varepsilon \downarrow 0} \frac{d \rho_{00}}{d \kappa}(\lambda+i \varepsilon) & =\frac{1}{\pi} \lim _{\varepsilon \downarrow 0} \Im\left(M_{\infty}\right)_{00}(\lambda+i \varepsilon) \\
& =\frac{1}{\pi L} \sum_{l=0}^{L-1} \lim _{\varepsilon \downarrow 0} \Im m_{j}(\lambda+i \varepsilon),
\end{aligned}
$$


$m_{j}$ a função de Weyl-Titchmarsh ligada à solução $l^{2}\left(\mathbb{Z}_{+}, \mathbb{C}\right)$ de $(2.4 .5)$, dada por

$$
m_{j}(z)=\frac{z_{j}}{2}+\sqrt{\frac{z_{j}^{2}}{4}-1}
$$

$z_{j}=z-2 \cos (2 \pi j / l), j=0,1, \ldots, L-1$. Como

$$
\lim _{\varepsilon \downarrow 0} \Im m_{j}(\lambda+i \varepsilon)=\left\{\begin{array}{ccc}
0 & \text { se } & \left|\lambda_{j}\right| \geq 2 \\
\sqrt{1-\lambda_{j}^{2} / 4} & \text { se } & \left|\lambda_{j}\right|<2
\end{array},\right.
$$

$\lambda_{j}=\lambda-2 \cos (2 \pi j / L)$, concluímos que a derivada de Radon-Nikodym (2.4.7) é estritamente positiva e finita para quase todo $\lambda$ (com respeito à medida de Lebesgue $\kappa$ ) contido no suporte essencial (2.4.3) de $\mathcal{J}_{0}$, e zero em seu complemento. A demonstração da proposição é concluída evocando-se o Lema D.3.8.

\subsubsection{Espectro essencial da matriz $\mathcal{J}_{\delta, \phi}$}

Uma questão natural que surge é se o espectro essencial da matriz $\mathcal{J}_{\delta, \phi}$ é, independentemente de $\delta \in(0,1)$ e de $\omega$, o mesmo que o de $\mathcal{J}_{0}$. Essa pergunta é respondida pelo

Teorema 2.4.5 (Teorema 2.3 de [CMW]) Seja $\mathcal{J}_{P, \phi}$ a matriz bloco-Jacobi associada ao operador $\Pi_{P, \phi}$ definido por (1.6) e que satisfaz as condições de contorno (1.16) e (1.17), com $P \in \mathcal{P}_{\delta, \beta}$ (isto é, a seqüência $P=\left\{p_{n}\right\}_{n \geq 0}$ satisfaz (1.9)-(1.11)). O espectro essencial de $\mathcal{J}_{\delta, \phi}$ é o intervalo (2.4.3) e, conseqüentemente,

$$
\sigma_{\text {ess }}\left(\mathcal{J}_{\delta, \phi}\right)=\sigma_{\text {ess }}\left(\mathcal{J}_{0}\right)
$$

segue para todo $\delta \in(0,1)$ e para todo $\omega \in \Gamma$.

Observação 2.4.6 O Teorema 2.4.5 é uma extensão do Teorema 2.1 de [MWGA]. Seguimos sua demonstração passo a passo.

Demonstração. Como a matriz bloco-Jacobi $\mathcal{J}_{\delta, \phi}$ pode ser tratada como uma perturbação de posto $L$ da matriz $\mathcal{J}_{\delta}$ (associada ao operador $\Pi_{\delta, 0}$ ), segue do Princípio de invariância de Weyl (Proposição B.2.14) que $\sigma_{\text {ess }}\left(\mathcal{J}_{\delta}\right)=\sigma_{\text {ess }}\left(\mathcal{J}_{\delta, \phi}\right)$. Assim, basta determinar o espectro essencial de $\mathcal{J}_{\delta}$.

Em primeiro lugar, provemos a inclusão $\sigma_{\text {ess }}\left(\mathcal{J}_{\delta}\right) \subseteq \sigma_{\text {ess }}\left(\mathcal{J}_{0}\right)$. Definamos para $u=$ $(u(x))_{x \in \Lambda} \in l^{2}\left(\Lambda, \mathbb{C}^{L}\right)$ os vetores coluna $2 L$-dimensionais

$$
\mathbf{u}_{k}=(u(k, 0), u(k+1,0), \ldots, u(k, L-1), u(k+1, L-1))
$$

e as matrizes $2 L \times 2 L$

$$
h_{k}^{L}=p_{k} I_{L} \otimes A_{2}+\frac{1}{2} A_{L} \otimes I_{2} .
$$


Desse modo, a forma quadrática associada a $\mathcal{J}_{\delta}$ pode ser escrita como

$$
\left(u, \mathcal{J}_{\delta} u\right)=\sum_{k=1}^{\infty} \mathbf{u}_{k} \cdot h_{k}^{L} \mathbf{u}_{k+1}+\sum_{m=0}^{L-1} u(0, m) u(0, m+1)
$$

o fator $1 / 2$ presente em (2.4.8) evita a contagem dupla de fatores em (2.4.9); a segunda soma presente em (2.4.9) corrige a contagem dos termos de interação entre os elementos da primeira coluna.

Seguimos a estratégia usada na Seção 2.1 para o cálculo dos autovalores de $h_{n}^{L}$. O polinômio característico de $h_{k}^{L}$ é simplesmente

$$
\begin{aligned}
& \operatorname{det}\left[h_{k}^{L}-\lambda I_{L} \otimes I_{2}\right]=\operatorname{det}\left[\left(F_{L}^{-1} \otimes I_{2}\right)\left(h_{k}^{L}-\lambda I_{L} \otimes I_{2}\right)\left(F_{L} \otimes I_{2}\right)\right] \\
&=\operatorname{det}\left[\operatorname{diag}\left(S_{m}-\lambda I_{2}\right)_{m=0}^{L-1}\right] \\
&=\prod_{m=0}^{L-1} \operatorname{det}\left[S_{m}-\lambda I_{2}\right], \\
& S_{m}=\left(\begin{array}{cc}
\cos (2 \pi m / L) & p_{k} \\
p_{k} & \cos (2 \pi m / L)
\end{array}\right),
\end{aligned}
$$

o que resulta nos autovalores $\lambda_{k, m}^{ \pm}= \pm p_{k}+\cos (2 \pi m / L), m=0, \ldots, L-1$. Inserindo a decomposição espectral de $h_{k}^{L}$,

$$
h_{k}^{L}=\sum_{m=0}^{L-1}\left(\lambda_{k, m}^{+} P_{k, m}^{+}+\lambda_{k, m}^{-} P_{k, m}^{-}\right)
$$

em (2.4.9) $\left(P_{k, m}^{ \pm}\right.$são os projetores nos auto-espaços associados aos autovetores de $\left.\lambda_{k, m}^{ \pm}\right)$, temos

$$
2 \lambda^{-} \leq \frac{\left(u, \mathcal{J}_{\delta} u\right)}{(u, u)} \leq 2 \lambda^{+}
$$

com

$$
\lambda^{+}=\sup _{k, m} \lambda_{k, m}^{+}=2
$$

e

$$
\lambda^{-}=\inf _{k, m} \lambda_{k, m}^{-}= \begin{cases}-2 & \text { se } L \text { for ímpar } \\ -1+\cos (\pi(L-1) / L) & \text { se } L \text { for par }\end{cases}
$$

para qualquer realização de $\omega$. Logo, os resultados acima, conjuntamente a (2.4.3), nos levam a $\sigma_{\text {ess }}\left(\mathcal{J}_{\delta}\right) \subseteq \sigma_{\text {ess }}\left(\mathcal{J}_{0}\right)$

A fim de demonstrarmos a inclusão $\sigma_{\text {ess }}\left(\mathcal{J}_{\delta}\right) \supseteq \sigma_{\text {ess }}\left(\mathcal{J}_{0}\right)$, usamos o critério de Weyl para o espectro essencial (Teorema B.2.13). Seja $\lambda_{m}(\varphi)=2 \cos \varphi-2 \cos (2 \pi m / L), \varphi \in[0, \pi)$, $m=0, \ldots, L-1$, e defina

$$
\psi_{n, m}=\psi_{n} \otimes v_{m}
$$

$\operatorname{com}\left(\psi_{n}\right)_{n(n-1) / 2+j}=(1 / \sqrt{n}) e^{i \varphi_{j}}$ para $j=1, \ldots, n$ e 0 de outra forma; $v_{m}$ é o $m-$ ésimo autovetor do operador $\Delta_{0}^{L}$ (vide equação (2.1.2)). Claramente $\psi_{n, m} \in l^{2}\left(\Lambda, \mathbb{C}^{2}\right)$ 
e $\left\{\lambda_{m}(\varphi)\right\}$ apresenta uma correspondência biunívoca com (2.4.3). Afirmamos que, para cada $m=0, \ldots, L-1$,

$$
\left\|\mathcal{J}_{\delta} \psi_{n, m}-\lambda_{m} \psi_{n, m}\right\| \leq c \frac{\ln n}{\sqrt{n}}
$$

segue com $c=c(\beta)$ independente de $n$. A fim de demonstrarmos (2.4.10), basta notar que $\mathcal{J}_{\delta} \psi_{n, m}-\lambda_{m} \psi_{n, m}$ consiste da ação de uma soma de matrizes locais em $\psi_{n, m}$, cada uma limitada em norma por um; $2 \mathrm{~L}$ delas envolvem os extremos $e^{i \varphi}$ e $e^{i n \varphi}$, e existem $O(\ln (n))$ matrizes não diagonais. A dependência $O(\ln (n))$ se deve ao fato da seqüência $\left(a_{j}^{\omega}\right)_{j \geq 1}$ satisfazer a condição $a_{j}+\omega_{j}-a_{j-1}-\omega_{j-1} \in\left(\beta^{j}-2 j+1, \beta^{j}+2 j-1\right)$, com no máximo $r$ pontos $a_{j}$ entre $[1, n] ; r$ é tal que

$$
r \leq \frac{\ln n}{\ln \beta}
$$

Repare que $A_{L} v_{m}=2 \cos (2 \pi m / L) v_{m}$ e esta parte do produto tensorial presente em $\mathcal{J}_{\delta}$ não tem efeito no processo limite. Poderíamos perguntar se a incerteza nas posições das barreiras poderia modificar esta estimativa. Uma vez que a esparsidade cresce exponencialmente e a incerteza nas posições cresce linearmente, $n$ satisfaz $\beta^{r+1}-r-1 \leq n \leq \beta^{r+1}$, o que não afeta a estimativa acima. Isso demonstra a inclusão $\sigma_{\text {ess }}\left(\mathcal{J}_{\delta}\right) \supseteq \sigma_{\text {ess }}\left(\mathcal{J}_{0}\right)$ e completa a demonstração do teorema.

Observação 2.4.7 Podemos obter os resultados do Teorema 2.4.5 apenas para o espectro de $\mathcal{J}_{\delta}$; basta substituir na demonstração acima o critério de Weyl para o espectro essencial pelo critério somente para o espectro (Teorema B.2.13). É possivel que ainda exista algum espectro discreto (vide a Seção B.2 para uma discussão a respeito as diferenças entre esses tipos de espectro), com o qual não nos preocupamos, fora do intervalo (2.4.3). 



\section{Capítulo 3}

\section{Existência de transição espectral}

Como vimos na Seção 2.3, devemos avaliar a natureza espectral da matriz bloco-Jacobi $\mathcal{J}_{\delta, \phi}$ a partir do elemento $\Omega_{00}$ da medida espectral matricial, que pode ser tratado como a "mistura simples"

$$
\Omega_{00}=\frac{1}{L} \sum_{j=0}^{L-1} \mu_{j}
$$

$\mu_{j}$ a medida espectral associada ao operador

$$
\left(\Delta_{P, \phi}^{j} u\right)_{n}=p_{n}(\omega) u_{n+1}+p_{n-1}(\omega) u_{n-1}+2 \cos (2 \pi j / L) u_{n},
$$

$j=0, \ldots, L-1$, com $P \in \mathcal{P}_{\delta, \beta}$ (ou seja, satisfazendo as relações (1.9)-(1.11)) e $\phi$ representando a condição de contorno (1.5).

Definimos a matriz de Jacobi $J_{P, \phi}^{j}$, a representação matricial do operador $\Delta_{P, \phi}^{j}$, como

$$
J_{P, \phi}^{j}=\left(\begin{array}{ccccc}
2 \cos (2 \pi j / L)+\tan \phi & p_{0} & 0 & 0 & \cdots \\
p_{0} & 2 \cos (2 \pi j / L) & p_{1} & 0 & \cdots \\
0 & p_{1} & 2 \cos (2 \pi j / L) & p_{2} & \cdots \\
0 & 0 & p_{2} & 2 \cos (2 \pi j / L) & \cdots \\
\vdots & \vdots & \vdots & \vdots & \ddots
\end{array}\right),
$$

(vide a Observação 1.4 para a discussão a respeito da inclusão da condição de contorno $\phi)$.

O conhecimento das principais características espectrais da matriz bloco-Jacobi $\mathcal{J}_{\delta, \phi}$ (como, por exemplo, seus tipos espectrais e a dimensão Hausdorff da medida $\Omega_{00}$ ), envolve, portanto, o comportamento coletivo das medidas espectrais $\mu_{j}$.

Tanto os resultados de Last e Simon (que permitem a caracterização da natureza espectral), presentes na Seção F.2, quanto os de Jitomirskaya e Last (que permitem a caracterização da parte singular do espectro), presentes no Apêndice $H$, necessitam do comportamento assintótico preciso da norma da matriz de transferência $T_{j}(n, \lambda)=$ 
$T_{j}(n, n-1 ; \lambda) T_{j}(n-1, n-2 ; \lambda) \cdots T_{j}(0,-1 ; \lambda)$ associada às soluções da equação de Schrödinger

$$
p_{n} u_{n+1}+p_{n+1} u_{n+1}+(2 \cos (2 \pi j / L)-\lambda) u_{n}=0
$$

$j=0, \ldots, L-1$, onde

$$
T_{j}(n, n-1 ; \lambda)=\left(\begin{array}{cc}
\frac{\lambda_{j}}{p_{n}} & \frac{-p_{n-1}}{p_{n}} \\
1 & 0
\end{array}\right) \equiv T\left(n, n-1 ; \lambda_{j}\right),
$$

$\lambda_{j} \equiv \lambda-2 \cos (2 \pi j / L)$

Observação 3.1 Repare que $J_{P, \phi}^{j}=J_{P, \phi}+2 \cos (2 \pi j / L) I, I$ o operador identidade em $l^{2}\left(\mathbb{Z}_{+}, \mathbb{C}\right)$. Podemos, dessa forma, reescrever a equação de Schrödinger (3.4) como

$$
p_{n} u_{n+1}+p_{n+1} u_{n+1}-\lambda_{j} u_{n}=0 .
$$

São, de fato, as ferramentas desenvolvidas por Marchetti et. al. em [MWGA] as adequadas ao nosso problema. Comecemos pelo

Teorema 3.2 Seja $J_{\delta, \phi}^{j}$ a matriz bloco-Jacobi associada ao operador $\Delta_{\delta, \phi}^{j}$ (3.2), $j=$ $0, \ldots, L-1$, com $P \in \mathcal{P}_{\delta, \beta}$ (isto é, a seqüência $P=\left\{p_{n}\right\}_{n \geq 0}$ satisfaz (1.9)- (1.11)). Então

$$
\sigma_{\text {ess }}\left(\mathcal{J}_{\delta, \phi}^{j}\right)=[-2+2 \cos (2 \pi j / L), 2+2 \cos (2 \pi j / L)] \equiv I_{j} .
$$

Observação 3.3 O Teorema 3.2 envolve uma pequena modificação no Teorema 2.1 de [MWGA].

Demonstração. Mais uma vez, podemos escrever a matriz $J_{\delta, \phi}^{j}$ como uma perturbação de posto um da matriz $J_{\delta}^{j}$ (associada ao operador $\Delta_{\delta, 0}^{j}$ ). A saber,

$$
J_{\delta, \phi}^{j}=J_{\delta}^{j}+\tan \phi \delta_{0}
$$

onde $\left(\delta_{0} u\right)_{n}=\delta_{n, 0} u_{0}$. Segue novamente do Princípio de invariância de Weyl (Proposição B.2.14) que $\sigma_{\text {ess }}\left(J_{\delta, \phi}^{j}\right)=\sigma_{\text {ess }}\left(J_{\delta}^{j}\right)$.

Provemos a inclusão $\sigma_{\text {ess }}\left(J_{\delta}^{j}\right) \subseteq I_{j}$. Seja $u=\left(u_{n}\right)_{n \geq 0} \in l^{2}\left(\mathbb{Z}_{+}, \mathbb{C}\right)$; podemos definir, em analogia a (2.4.9), a forma quadrática

$$
\left(u, J_{\delta}^{j} u\right)=\sum_{n=0}^{\infty}\left(\begin{array}{ll}
u_{n} & u_{n+1}
\end{array}\right) h_{n}^{j}\left(\begin{array}{c}
u_{n} \\
u_{n+1}
\end{array}\right),
$$

onde

$$
h_{n}^{j}=\left(\begin{array}{cc}
\cos (2 \pi j / L) & p_{n} \\
p_{n} & \cos (2 \pi j / L)
\end{array}\right)
$$


Inserindo a decomposição espectral de $h_{n}^{j}$,

$$
h_{n}^{j}=\lambda_{n}^{+} P_{n}^{+}+\lambda_{n}^{-} P_{n}^{-},
$$

em (3.6) ( $P_{n}^{ \pm}$são os projetores nos auto-espaços associados aos autovetores de $\left.\lambda_{n}^{ \pm}\right)$, temos

$$
2 \lambda^{-} \leq \frac{\left(u, \mathcal{J}_{\delta}^{j} u\right)}{(u, u)} \leq 2 \lambda^{+},
$$

com

$$
\lambda^{ \pm}=\sup _{n} \lambda_{n}^{ \pm}= \pm 1+\cos (2 \pi j / L),
$$

para qualquer realização de $\omega$. Assim, $\sigma_{\text {ess }}\left(\mathcal{J}_{\delta}^{j}\right) \subseteq\left[2 \lambda^{-}, 2 \lambda^{+}\right]=[-2+2 \cos (2 \pi j / L), 2+$ $2 \cos (2 \pi j / L)]$.

Demonstramos a inclusão $\sigma_{\text {ess }}\left(\mathcal{J}_{\delta}^{j}\right) \supseteq I_{j}$ mais uma vez evocando o critério de Weyl (Teorema B.2.13). Seja $\lambda_{j}=2 \cos \varphi_{j}=\lambda-2 \cos (2 \pi j / L), \varphi_{j} \in[0, \pi)$; caso definamos $\left(\psi_{n}\right)_{n(n-1) / 2+j}=(1 / \sqrt{n}) e^{i \varphi_{j}}$ para $j=1, \ldots, n$ e 0 de outra forma, é possível mostrar que (vide a Observação 3.1)

$$
\left\|\left(J_{\delta} \psi_{n}^{j}-\lambda_{j}\right) \psi_{n}^{j}\right\| \leq c \frac{\ln n}{\sqrt{n}}
$$

segue com $c=c(\beta)$ independente de $n$ e de $\omega$ (vide a demonstração do Teorema 2.4.5). Isso demonstra a inclusão $\sigma_{\text {ess }}\left(\mathcal{J}_{\delta}^{j}\right) \supseteq I_{j}$. Como todos os resultados acima independem de $j=0,1, \ldots, L-1$, concluímos a demonstração do teorema.

\subsection{Matrizes de transferência e variáveis de Prüfer adaptadas}

Prosseguimos com determinação das matrizes de transferência. Tendo em vista as relações (1.9)-(1.11), existem apenas três situações a serem consideradas: para $n \notin\left\{a_{j}^{\omega}, a_{j+1}^{\omega}\right\}$, $j \geq 1$, (3.5) é igual à matriz de transferência livre

$$
T_{0}\left(\lambda_{j}\right)=\left(\begin{array}{cc}
\lambda_{j} & -1 \\
1 & 0
\end{array}\right) ;
$$

para $n=a_{j}^{\omega}+1$,

$$
T_{+}\left(\lambda_{j}\right)=\left(\begin{array}{cc}
\lambda_{j} & -1+\delta \\
1 & 0
\end{array}\right)
$$

finalmente, para $n=a_{j}$,

$$
T_{-}\left(\lambda_{j}\right)=\left(\begin{array}{cc}
\frac{\lambda_{j}}{1-\delta} & -\frac{1}{1-\delta} \\
1 & 0
\end{array}\right)
$$


Seja $\lambda=2 \cos \varphi_{j}+2 \cos (2 \pi j / L)$, com $\varphi_{j} \in(0, \pi)$, uma possível parametrização do espectro essencial da matriz de Jacobi $J_{\delta, \phi}^{j}($ como visto no Teorema 3.2). É bastante simples mostrar que a matriz de transferência livre $T_{0}\left(\lambda_{j}\right)$ é semelhante a uma matriz de rotação horária $R\left(\varphi_{j}\right)$ :

$$
U_{j} T_{0}\left(\lambda_{j}\right) U_{j}^{-1}=\left(\begin{array}{cc}
\cos \varphi_{j} & \sin \varphi_{j} \\
-\sin \varphi_{j} & \cos \varphi_{j}
\end{array}\right)=R\left(\varphi_{j}\right)
$$

onde

$$
U_{j} \equiv\left(\begin{array}{cc}
0 & \sin \varphi_{j} \\
1 & -\cos \varphi_{j}
\end{array}\right)
$$

Observação 3.1.1 Repare que a matriz $U_{j}$ não é univocamente determinada, uma vez que qualquer outra $U_{j}^{\prime}=H U_{j}$, com $H$ uma matriz que comute com $R$, satisfaz (3.1.4).

Sendo o produto de matrizes de rotação também uma rotação, obtemos

$$
U_{j} T\left(n ; \lambda_{j}\right) U_{j}^{-1}=R\left(\left[n-a_{k}^{\omega}-1\right] \varphi_{j}\right) U_{j} T_{+-}^{j} U_{j}^{-1} R\left(\left[a_{k}^{\omega}-a_{k-1}^{\omega}-2\right] \varphi_{j}\right) \cdots U_{j} T_{+-}^{j} U_{j}^{-1} R\left(\left[a_{1}^{\omega}-1\right] \varphi_{j}\right)
$$

como a transformação de similaridade por $U_{j}$ da matriz de transferência $T\left(n ; \lambda_{j}\right) ; k$ é um número inteiro definido de tal modo que $a_{k}^{\omega} \leq n<a_{k+1}^{\omega}$ e $T_{+-}^{j}:=T_{+}\left(\lambda_{j}\right) T_{-}\left(\lambda_{j}\right)$.

É bastante conveniente introduzir em (3.1.6) a matriz $P_{+-}$, definida a partir de

$$
R\left(\varphi_{j}\right) P_{+-}^{j} R\left(\varphi_{j}\right)=U_{j} T_{+-}^{j} U_{j}^{-1} .
$$

Explicitamente, temos das definições de $U_{j}$ (equação (3.1.5)) e das matrizes de transferência $T_{+}\left(\lambda_{j}\right)$ e $T_{-}\left(\lambda_{j}\right)$ (equações (3.1.2) e (3.1.3), respectivamente),

$$
R\left(-\varphi_{j}\right) U_{j} T_{+}\left(\lambda_{j}\right) U_{j}^{-1}=\left(\begin{array}{cc}
p & 0 \\
(1-p) \cot \varphi_{j} & 1
\end{array}\right)
$$

$\mathrm{e}$

$$
U_{j} T_{-}\left(\lambda_{j}\right) U_{j}^{-1} R\left(-\varphi_{j}\right)=\left(\begin{array}{cc}
1 & 0 \\
(1-p) / p \cot \varphi_{j} & 1 / p
\end{array}\right)
$$

de onde obtemos

$$
P_{+-}^{j}=\left(\begin{array}{cc}
p & 0 \\
(1 / p-p) \cot \varphi_{j} & 1 / p
\end{array}\right)
$$

Podemos, portanto, reescrever (3.1.6) como

$$
U_{j} T\left(n ; \lambda_{j}\right) U_{j}^{-1}=R\left(\left[n-a_{k}^{\omega}\right] \varphi_{j}\right) P_{+-}^{j} R\left(\left[a_{k}^{\omega}-a_{k-1}^{\omega}\right] \varphi_{j}\right) \cdots P_{+-}^{j} R\left(a_{1}^{\omega} \varphi_{j}\right) .
$$

A matriz $P_{+-}^{j}$ nos permite tratar, portanto, as perturbações ao operador livre, que se dão nas "ligações" entre os sítios, como sendo pontuais. Isso representa uma vantagem significativa na determinação das variáveis de Prüfer, como veremos a seguir. 
A estrutura presente na equação (3.1.8) sugere uma redefinição das variáveis de Prüfer apresentadas na relação (F.3.1). De fato, se definirmos os vetores

$$
\mathbf{v}_{k}^{j}=\left(R_{k-1}^{j} \cos \theta_{k}^{\omega, j}, R_{k-1}^{j} \sin \theta_{k}^{\omega, j}\right)
$$

e

$$
\tilde{\mathbf{v}}_{k}^{j}=\left(R_{k}^{j} \cos \tilde{\theta}_{k}^{\omega, j}, R_{k}^{j} \sin \tilde{\theta}_{k}^{\omega, j}\right),
$$

então as variáveis de Prüfer $\left(R_{k}^{j}, \theta_{k}^{j}\right)_{k \geq 0}$ (omitimos a dependência de $\omega$ a fim de não sobrecarregar a notação) satisfazem uma relação de recorrência induzida por

$$
\mathbf{v}_{k}^{j}=R\left(\left(a_{k}^{\omega}-a_{k-1}^{\omega}\right) \varphi_{j}\right) \tilde{\mathbf{v}}_{k-1}^{j}
$$

e por

$$
\tilde{\mathbf{v}}_{k}^{j}=P_{+-}^{j} \mathbf{v}_{k}^{j},
$$

$\operatorname{com} \mathbf{v}_{1}^{j}=R\left(a_{1}^{\omega} \varphi_{j}\right) \tilde{\mathbf{v}}_{0}^{j}$,

$$
\tilde{\mathbf{v}}_{0}^{j}\left(\theta_{0}^{j}\right)=R_{0}^{j}\left(\begin{array}{c}
\cos \theta_{0}^{j} \\
\sin \theta_{0}^{j}
\end{array}\right)=U_{j}\left(\begin{array}{c}
\cos \phi \\
\sin \phi
\end{array}\right)=\left(\begin{array}{c}
\sin \varphi_{j} \sin \phi \\
\cos \phi-\cos \varphi_{j} \sin \phi
\end{array}\right)
$$

(como em (F.3.5)), onde $\left(R_{0}^{j}\right)^{2}=1-\cos \varphi_{j} \sin 2 \phi$. Desse modo, se $\mathbf{u}_{\mathbf{n}}=\left(u_{n}, u_{n-1}\right)$ representa a solução da equação de Schrödinger (3.4) sujeita às condições iniciais $\mathbf{u}_{\mathbf{0}}=$ $(\cos \phi, \sin \phi)$, então a relação (F.3.4) deve ser reescrita, para todo $k \geq 0$, como

$$
R\left(\varphi_{j}\right) \tilde{\mathbf{v}}_{k}^{j}=U_{j} \mathbf{u}_{a_{k+1}} .
$$

Observação 3.1.2 1. Esta definição de ângulo de Prüfer sugerida por Marchetti et. al. difere um pouco da apresentada por Pearson em $[P]$ e por outros autores. Por $\theta_{n}^{j}$, nos referimos ao ângulo de Prüfer no sítio $a_{n}^{\omega}$ imediatamente anterior a $n-$ ésima barreira. A definição de Pearson se dá no ponto $a_{n}^{\omega}+1$, isto é, no ponto imediatamente consecutivo à barreira.

2. Vale destacar que esta nova definição de variáveis de Prüfer incorpora o fato do raio permanecer constante após uma iteração com a matriz de transferência livre, cujo único efeito é, como veremos a seguir, produzir um deslocamento no ângulo por um fator $\varphi_{j}$. Em modelos esparsos como esse, a grande maioria das iterações produz exatamente este efeito.

\subsubsection{Determinação do comportamento assintótico da seqüência $\left(R_{n}^{j}\left(\theta_{0}^{j}\right)\right)_{n \geq 0}$}

O Teorema F.3.3 nos mostra a importância de se estudar o comportamento assintótico da seqüência $\left(R_{n}^{j}\left(\theta_{0}^{j}\right)\right)_{n \geq 0}$ dos raios de Prüfer $\left(\theta_{0}^{j}\right.$ representa o ângulo de Prüfer inicial). É exatamente isso que buscamos fazer agora. 
Segue imediatamente das definições do vetores $\mathbf{v}^{j}$ e $\tilde{\mathbf{v}}^{j}$ (equações (3.1.9) e (3.1.10)), da matriz de transferência $T\left(n ; \lambda_{j}\right)$, e da norma $\|\cdot\|_{U_{j}}$, a identidade

$$
\left\|T\left(n ; \lambda_{j}\right) \tilde{\mathbf{v}}_{0}^{j}\left(\theta_{0}^{j}\right)\right\|_{U_{j}}=\left\|T\left(a_{N}^{\omega}+1 ; \lambda_{j}\right) \tilde{\mathbf{v}}_{0}^{j}\left(\theta_{0}^{j}\right)\right\|_{U_{j}}=R_{N}^{j}\left(\theta_{0}^{j}\right)
$$

(equação 3.8 de [MWGA]), válida para todo $n$ tal que $a_{N}^{\omega} \leq n<a_{N+1}^{\omega}$.

Explicitamente,

$$
\begin{aligned}
R_{N}^{j}\left(\begin{array}{c}
\cos \tilde{\theta}_{N}^{j} \\
\sin \tilde{\theta}_{N}^{j}
\end{array}\right) & =R_{N-1}^{j} P_{+-}^{j}\left(\begin{array}{c}
\cos \theta_{N}^{j} \\
\sin \theta_{N}^{j}
\end{array}\right) \\
& =R_{N-1}^{j}\left(\begin{array}{c}
p \cos \theta_{N}^{j} \\
(1 / p-p) \cot \varphi_{j} \cos \theta_{N}^{j}+\sin \theta_{N}^{j} / p
\end{array}\right)
\end{aligned}
$$

(omitimos a dependência de $R_{N}^{j}$ e $R_{N+1}^{j}$ com respeito ao ângulo de Prüfer inicial $\theta_{0}^{j}$ ) segue das definições (3.1.9), (3.1.10) e de (3.1.12). Obtemos, ao tomar as normas dos vetores acima, a relação

$$
\left(R_{N}^{j}\right)^{2}=\frac{\left(R_{N-1}^{j}\right)^{2}}{p^{2}} f\left(p, \theta_{N}^{j}\right)
$$

onde

$$
f(p, \theta):=p^{4} \cos ^{2} \theta+\left(\sin \theta+\left(1-p^{2}\right) \cot \varphi_{j} \cos \theta\right)^{2},
$$

de modo que

$$
\left(R_{N}^{j}\right)^{2}=\left(R_{0}^{j}\right)^{2} \prod_{n=1}^{N}\left(\frac{R_{n}^{j}}{R_{n-1}^{j}}\right)^{2}=\left(R_{0}^{j}\right)^{2}\left(\frac{1}{p^{2}} \exp \left\{\frac{1}{N} \sum_{n=1}^{N} \ln f\left(p, \theta_{n}^{j}\right)\right\}\right)^{N} .
$$

Chegamos de (3.1.11) à identidade

$$
\begin{aligned}
R_{N}^{j}\left(\begin{array}{c}
\cos \theta_{N+1}^{j} \\
\sin \theta_{N+1}^{j}
\end{array}\right) & =R_{N}^{j} R\left(\left(a_{N+1}^{\omega}-a_{N}^{\omega}\right) \varphi_{j}\right)\left(\begin{array}{c}
\cos \tilde{\theta}_{N}^{j} \\
\sin \tilde{\theta}_{N}^{j}
\end{array}\right) \\
& =R_{N}^{j}\left(\begin{array}{c}
\cos \left(\tilde{\theta}_{N}^{j}-\left(a_{N+1}^{\omega}-a_{N}^{\omega}\right) \varphi_{j}\right) \\
\sin \left(\tilde{\theta}_{N}^{j}-\left(a_{N+1}^{\omega}-a_{N}^{\omega}\right) \varphi_{j}\right)
\end{array}\right),
\end{aligned}
$$

que conjuntamente a (3.1.16) resulta na relação de recorrência

$$
\theta_{N+1}^{j}=g\left(p, \theta_{N}^{j}\right)-\left(a_{N+1}^{\omega}-a_{N}^{\omega}\right) \varphi_{j}, \quad N \geq 2,
$$

para os ângulos de Prüfer $\left(\theta_{N}^{j}\right)_{N \geq 0}, \operatorname{com} \theta_{1}^{j}=\theta_{0}^{j}-a_{1}^{\omega} \varphi_{j}$;

$$
g(\theta)=\tan ^{-1}\left((\tan \theta+\cot \varphi) /(1-\delta)^{2}-\cot \varphi\right)
$$

é uma função monótona crescente que mapeia o intervalo $[-\pi / 2, \pi / 2)$ nele mesmo. 
A determinação do comportamento assintótico exato da seqüência $\left(R_{n}^{j}\left(\theta_{0}^{j}\right)\right)_{n>0}$ dos raios de Prüfer envolve, portanto, uma estimativa do comportamento assintótico da soma

$$
\frac{1}{N} \sum_{n=1}^{N} \ln f\left(p, \theta_{n}^{j}\right)=\frac{1}{N} \sum_{n=1}^{N} \ln \left(p^{4} \cos ^{2} \theta_{n}^{j}+\left[\sin \theta_{n}^{j}+\left(1-p^{2}\right) \cot \varphi_{j} \cos \theta_{n}^{j}\right]^{2}\right),
$$

que por sua vez, depende de propriedades de distribuição da seqüência $\left(\theta_{n}^{j}\right)_{n \geq 0}$ dos ângulos de Prüfer. Necessitamos de algumas ferramentas matemáticas para uma análise detalhada dessa questão; começamos pela

Definição 3.1.3 Um sequência $w=\left(x_{n}\right)_{n \geq 1}$ de números reais $x_{n} \in[0, \pi)$ é dita uniformemente distribuída módulo $\pi(\mathrm{u} . \mathrm{d} \cdot \bmod \pi)$ se, para todo par a, $b$ de números reais que satisfizerem $0 \leq a<b \leq \pi$, tivermos

$$
\lim _{N \rightarrow \infty} \frac{A([a, b) ; N ; w)}{N}=b-a,
$$

onde definimos $A([a, b) ; N ; w)$ como o número de termos $x_{n}, 1 \leq n \leq N$, para os quais $x_{n} \in[a, b)$.

Em outras palavras, a seqüência $w=\left(x_{n}\right)_{n \geq 1}$ é u.d. $\bmod \pi$ se esta for igualmente distribuída, em partes fracionárias, em subintervalos semi-abertos do intervalo $[0, \pi)$. Fica claro que podemos reescrever (3.1.23) na forma

$$
\lim _{N \rightarrow \infty} \frac{1}{N} \sum_{n=1}^{N} \chi_{[a, b)}\left(\left\{x_{n}\right\}\right)=\frac{1}{\pi} \int_{0}^{\pi} \chi_{[a, b)}(x) d x
$$

onde $\{a\}$ designa a parte fracionária do número real $a$ com respeito a $\pi$ (ou seja, $a=m \pi+$ $\{a\}, m \in \mathbb{Z}$ ). Esta observação, juntamente com uma importante técnica de aproximação, nos conduz ao seguinte critério.

Teorema 3.1.4 (Teorema 1.1 de $[\mathbf{K N}]$ ) A seqüência $w=\left(x_{n}\right)_{n \geq 1}$ de números reais $x_{n} \in[0, \pi)$ é u.d. $\bmod \pi$ se, e somente se, para toda função real contínua $f$ definida no intervalo fechado $I=[0, \pi]$, tivermos

$$
\lim _{N \rightarrow \infty} \frac{1}{N} \sum_{n=1}^{N} f\left(\left\{x_{n}\right\}\right)=\frac{1}{\pi} \int_{0}^{\pi} f(x) d x .
$$

Observação 3.1.5 O Teorema 3.1.4 corresponde, na realidade, a uma ligeira variação do Teorema 1.1 de $[K N]$, referente à troca do intervalo $[0,1)$ pelo intervalo $[0, \pi)$. O nosso interesse no intervalo $[0, \pi)$ se deve ao fato da função $f(p, \theta)$, definida por (3.1.17), ser uma função Riemann integrável e periódica de período $\pi$, como veremos.

Demonstração. Sejam $w=\left(x_{n}\right)_{n \geq 1}$ u.d. $\bmod \pi$ e $f(x)=\sum_{i=0}^{k-1} d_{i} \chi_{\left[a_{i}, a_{i+1}\right]}(x)$ uma função simples em $I$, onde $0=a_{0}<a_{1}<\ldots<a_{k}=\pi$. Temos de (3.1.24) que a relação 
(3.1.25) é satisfeita para toda função $f$ assim definida. Assumimos agora que $f$ é uma função real e contínua definida em $I$. Dado um $\varepsilon>0$ qualquer, existem, pela definição de integral de Riemann, duas funções simples $f_{1}$ e $f_{2}$ tais que $f_{1}(x) \leq f(x) \leq f_{2}(x)$ para todo $x \in I$, e que $\int_{0}^{\pi}\left(f_{2}(x)-f_{1}(x)\right) d x \leq \varepsilon$, de onde obtemos

$$
\begin{aligned}
\frac{1}{\pi} \int_{0}^{\pi} f(x) d x-\varepsilon & \leq \frac{1}{\pi} \int_{0}^{\pi} f_{1}(x) d x=\lim _{N \rightarrow \infty} \frac{1}{N} \sum_{n=1}^{N} f_{1}\left(x_{n}\right) \\
& \leq \liminf _{N \rightarrow \infty} \frac{1}{N} \sum_{n=1}^{N} f\left(x_{n}\right) \leq \limsup _{N \rightarrow \infty} \frac{1}{N} \sum_{n=1}^{N} f\left(x_{n}\right) \\
& \leq \lim _{N \rightarrow \infty} \frac{1}{N} \sum_{n=1}^{N} f_{2}\left(x_{n}\right)=\frac{1}{\pi} \int_{0}^{\pi} f_{2}(x) d x \leq \frac{1}{\pi} \int_{0}^{\pi} f(x) d x+\varepsilon
\end{aligned}
$$

concluímos, portanto, que a equação (3.1.25) é satisfeita caso $f$ seja uma função contínua.

Por outro lado, seja $w=\left(x_{n}\right)_{n \geq 1}$ uma dada seqüência, e suponha que a equação (3.1.25) seja satisfeita para toda função real contínua $f$ definida no intervalo $I$. Seja $[a, b)$ um subintervalo arbitrário de $I$. Dado um $\varepsilon>0$ qualquer, existem duas funções contínuas, $g_{1}$ e $g_{2}$, tais que $g_{1}(x) \leq \chi_{[a, b)}(x) \leq g_{2}(x)$ segue para todo $x \in I$, ao mesmo tempo que $\frac{1}{\pi} \int_{0}^{\pi}\left(g_{2}(x)-g_{1}(x)\right) d x \leq \varepsilon$. Temos então

$$
\begin{aligned}
b-a-\varepsilon & \leq \frac{1}{\pi} \int_{0}^{\pi} g_{2}(x) d x-\varepsilon \leq \frac{1}{\pi} \int_{0}^{\pi} g_{1}(x) d x=\lim _{N \rightarrow \infty} \frac{1}{N} \sum_{n=1}^{N} g_{1}\left(x_{n}\right) \\
& \leq \liminf _{N \rightarrow \infty} \frac{A([a, b) ; N)}{N} \leq \limsup _{N \rightarrow \infty} \frac{A([a, b) ; N)}{N} \leq \lim _{N \rightarrow \infty} \frac{1}{N} \sum_{n=1}^{N} g_{2}\left(x_{n}\right) \\
& =\frac{1}{\pi} \int_{0}^{\pi} g_{2}(x) d x \leq \frac{1}{\pi} \int_{0}^{\pi} g_{1}(x) d x+\varepsilon \leq b-a+\varepsilon .
\end{aligned}
$$

Como $\varepsilon$ é arbitrariamente pequeno, obtemos (3.1.23). Isso conclui a demonstração do teorema.

O Teorema 3.1.4 nos fornece, portanto, um critério que nos permite substituir, no limite assintótico $N \rightarrow \infty$, a soma (3.1.22) pela integral

$$
\frac{1}{\pi} \int_{0}^{\pi} \ln f(p, \theta) d \theta
$$

desde que a seqüência $\left(\theta_{n}^{j}\right)_{n \geq 1}$ dos ângulos de Prüfer seja u. d. $\bmod \pi$ e que $\ln f(p, \theta)$, com $f(p, \theta)$ definida por (3.1.17) seja uma função Riemann integrável e periódica de período $\pi$. Suponhamos, por hora, a

Hipótese 3.1.6 A seqüência $\left(\theta_{n}^{j}\right)_{n \geq 1}$ dos ângulos de Prüfer definidos pela relação de recorrência (3.1.20) é u.d. $\bmod \pi$ para $\varphi_{j} \in[0, \pi) \backslash A_{j}$, onde $A_{j}$ é um conjunto de medida de Lebesgue nula. 
Entenderemos o porquê do conjunto $A_{j}$ quando efetivamente demonstrarmos a validade da Hipótese 3.1.6.

Lema 3.1.7 A função $h(\theta):=\ln \left[p^{4} \cos ^{2} \theta+\left(\sin \theta+\left(1-p^{2}\right) \cot \varphi_{j} \cos \theta\right)^{2}\right]$ é uma função Riemann-integrável periódica de período $\pi$, cujo valor médio é dado por

$$
\frac{1}{\pi} \int_{0}^{\pi} g(p, \theta) d \theta=\ln \left(\frac{\left(1-p^{2}\right)^{2} \operatorname{cosec}^{2} \varphi_{j}}{4}+p^{2}\right) .
$$

Observação 3.1.8 O conteúdo do Lema 3.1.7 está contido na Seção 4 de [MWGA].

Demonstração. Podemos reescrever $h(\theta)$, através do uso de identidades trigonométricas simples, como

$$
h(\theta)=\ln (a+b \cos 2 \theta+c \sin 2 \theta)
$$

com

$$
\begin{aligned}
& 2 a=\left(1-p^{2}\right)^{2} \cot ^{2} \varphi_{j}+1+p^{4}, \\
& 2 b=\left(1-p^{2}\right)^{2} \cot ^{2} \varphi_{j}-1+p^{4}, \\
& c=\left(1-p^{2}\right) \cot \varphi_{j} .
\end{aligned}
$$

Isso mostra que $h(\theta)$ é periódica de período $\pi$. Resta demonstrarmos que $h(\theta)$ é Riemann-integrável. Um cálculo explícito nos mostra que

$$
\begin{aligned}
a+b \cos (2 \theta)+c \sin (2 \theta) & \geq \min _{\theta \in[0, \pi)}(a+b \cos (2 \theta)+c \sin (2 \theta)) \\
& =a-\sqrt{b^{2}+c^{2}}=a-\sqrt{a^{2}-p^{4}}>0,
\end{aligned}
$$

já que $0<b^{2}+c^{2}=a^{2}-p^{4}$ se $0<p<1 ; h(\theta)$ é, portanto, contínua no intervalo $[0, \pi)$, e assim, Riemann integrável.

Por fim, temos da página 546 de [PBM]

$$
\begin{aligned}
\frac{1}{\pi} \int_{0}^{\pi} h(\theta) d \theta & =\ln \left(\frac{a+\sqrt{a^{2}-b^{2}-c^{2}}}{2}\right)=\ln \left(\frac{a+p^{2}}{2}\right) \\
& =\ln \left(\frac{\left(1-p^{2}\right)^{2} \operatorname{cosec}^{2} \varphi_{j}+\left(1+p^{2}\right)^{2}-\left(1-p^{2}\right)^{2}}{4}\right) \\
& =\ln \left(\frac{\left(1-p^{2}\right)^{2} \operatorname{cosec}^{2} \varphi_{j}}{4}+p^{2}\right)
\end{aligned}
$$

exatamente (3.1.27). Isso encerra a demonstração.

O Lema 3.1.7, conjuntamente ao Teorema 3.1.4, fornecem um valor preciso para o limite assintótico da "média temporal" $\frac{1}{N} \sum_{n=1}^{N} \ln f\left(p, \theta_{n}^{j}\right)$, sob a hipótese de distribuição uniforme mod $\pi$ (no sentido da Definição 3.1.3) da seqüência $\left(\theta_{n}^{j}\right)_{n \geq 1}$ dos ângulos de Prüfer. 
De fato, a desigualdade de Koksma (Teorema 5.1 do capítulo 2 de [KN]) nos permite avaliar o erro que cometemos ao substituir a soma finita (3.1.22) pela integral (3.1.26); a saber,

$$
\left|\frac{1}{N} \sum_{n=1}^{N} \ln f\left(p, \theta_{n}^{j}\right)-\frac{1}{\pi} \int_{0}^{\pi} \ln f(p, \theta) d \theta\right| \leq V(h) D_{N}^{*}
$$

onde

$$
D_{N}^{*}\left(\theta^{j}\right)=D_{N}^{*}\left(\theta_{1}^{j}, \ldots, \theta_{N}^{j}\right)=\sup _{0<\alpha \leq \pi}\left|\frac{A([0, \alpha) ; N)}{N}-\alpha\right|
$$

é a chamada discrepância ${ }^{\dagger}$ da seqüência $\left(\theta_{n}^{j}\right)_{n \geq 1} \mathrm{e}$

$$
V(h)=\sum_{n=1}^{N-1}\left|\ln f\left(p, \theta_{n+1}^{j}\right)-\ln f\left(p, \theta_{n}^{j}\right)\right| \leq C
$$

é a variação constante da função $h$ (é possível mostrar que $\sup _{\theta \in[0, \pi)}\left|h^{\prime}(\theta)\right|<\infty$; vide a demonstração do Lema 3.1.7).

A discrepância $D_{N}^{*}(\omega)$ de uma seqüência de números reais $\omega$ é uma medida de quanto a distribuição desta seqüência se desvia da uniforme. De fato, temos o

Teorema 3.1.9 (Corolário 1.1 do Capítulo 2 de [KN]) A seqüência $\omega$ é u.d.mod $\pi$ se, e somente se $\lim _{N \rightarrow \infty} D_{N}^{*}(\omega)=0$.

Demonstração. A suficiência da condição é óbvia. Escolhemos, a fim de mostrar a necessidade, um inteiro $m \geq 2$. Para $0 \leq k \leq m-1$, definamos $I_{k}=[k / m,(k+1) / m)$. Sendo $\omega$ u.d. $\bmod \pi$, existe um inteiro positivo $N_{0}=N_{0}(m)$ tal que, para $N \geq N_{0}$ e para todo $k=0,1, \ldots, m-1$, temos

$$
\frac{1}{m}\left(1-\frac{1}{m}\right) \leq \frac{A\left(I_{k} ; N\right)}{N} \leq \frac{1}{m}\left(1+\frac{1}{m}\right) .
$$

Considere agora um subintervalo arbitrário $J=[0, \alpha) \subset[0, \pi)$. Claramente, existem intervalos $J_{1}=\bigcup_{k=0}^{k_{1}} I_{k}$ e $J_{2}=\bigcup_{k=0}^{k_{2}} I_{k}$ tais que $J_{1} \subseteq J \subseteq J_{2}$, com $|J|-\left|J_{1}\right|,\left|J_{2}\right|-|J|<2 / m$ (desde que $\left(k_{1}+1\right) / m \leq \alpha \leq\left(k_{2}+1\right) / m$ ). Obtemos das desigualdades acima, para todo $N \geq N_{0}$

$$
\left|J_{1}\right|\left(1-\frac{1}{m}\right) \leq \frac{A\left(J_{1} ; N\right)}{N} \leq \frac{A(J ; N)}{N} \leq \frac{A\left(J_{2} ; N\right)}{N} \leq\left|J_{2}\right|\left(1+\frac{1}{m}\right),
$$

e conseqüentemente,

$$
\left(|J|-\frac{2}{m}\right)\left(1-\frac{1}{m}\right) \leq \frac{A(J ; N)}{N} \leq\left(|J|-\frac{2}{m}\right)\left(1+\frac{1}{m}\right) .
$$

†Para uma seqüência infinita $x$ de números reais, $D_{N}^{*}(x)$ se refere à discrepância do segmento formado pelos primeiros $N$ termos de $x$. 
Obtemos, usando $|J| \leq \pi$

$$
-\frac{\pi+2}{m}+\frac{2}{m^{2}}<\frac{A(J ; N)}{N}-|J|<\frac{\pi+2}{m}+\frac{2}{m^{2}},
$$

para todo $N \geq N_{0}$. Uma vez que os limitantes na expressão acima independem de $J$, segue da definição de discrepância que $D_{N}^{*}(\omega) \leq(\pi+2) / m+2 / m^{2}$, para todo $N \geq N_{0}$. No entanto, $(\pi+2) / m+2 / m^{2}$ pode ser feito arbitrariamente pequeno, e assim completamos a demonstração.

Observação 3.1.10 A demonstração do Teorema 3.1.9 corresponde a uma pequena variante (para intervalos do tipo $[0, \alpha)$ em vez de $[\alpha, \beta)$ ) da demonstração do Teorema 1.1 do Capítulo 2 de $[K N]$.

O seguinte resultado é fornecido pela relação (4.19) de [MWGA].

Lema 3.1.11 Seja $\left(R_{n}^{j}\left(\theta_{0}^{j}\right)\right)_{n \geq 1}$ a seqüência dos raios de Prüfer associados à solução da equação (3.4) que satisfaz o par de condições iniciais $\mathbf{v}_{0}^{j}=(\cos \alpha, \sin \alpha)\left(\mathbf{v}_{0}^{j}\right.$ e $\theta_{0}^{j}$ se relacionam através de (3.1.13)). Suponha a validade da Hipótese 3.1.6. Então, obtemos para $\varphi_{j} \in[0, \pi) \backslash A_{j}\left(A_{j}\right.$ é um conjunto de medida de Lebesgue nula), as desigualdades

$$
C_{N, j}^{-1} r_{j}^{N} \leq\left(R_{N}^{j}\left(\theta_{0}^{j}\right)\right)^{2} \leq C_{N, j} r_{j}^{N}
$$

onde $C_{N, j}$ representa um número real tal que $C_{N, j}>1$ e $\lim _{N \rightarrow \infty} C_{N, j}^{1 / N}=\left(R_{0}^{j}\right)^{2}$, com $\left(R_{0}^{j}\right)^{-2}=1-\cos \varphi_{j} \sin 2 \phi, e$

$$
r_{j}=1+\frac{\left(1-p^{2}\right)^{2}}{4 p^{2}} \operatorname{cosec}^{2} \varphi_{j}
$$

Demonstração. As desigualdades (3.1.29) são diretamente obtidas a partir das equações (3.1.18), (3.1.27), da estimativa (3.1.28) e da Hipótese 3.1.6.

Como, pela Hipótese 3.1.6 e pelo Teorema 3.1.9, $\lim _{N \rightarrow \infty} D_{N}^{*}\left(\theta_{0}^{j}\right)=0$, fica claro que $\lim _{N \rightarrow \infty} C_{N, j}^{1 / N}=\left(R_{0}^{j}\left(\theta_{0}^{j}\right)\right)^{2}$.

É importante salientar que as desigualdades (3.1.29) são satisfeitas para quase todo par de condições iniciais $\tilde{\mathbf{v}}_{0}^{j}\left(\theta_{0}^{j}\right)$ (vide (3.1.13)), ou seja, a menos de um conjunto de medida de Lebesgue nula. É provável que, para esse conjunto de condições iniciais com medida nula, a seqüência $\left(R_{n}^{j}\left(\theta_{0}^{j}\right)\right)_{n \geq 1}$ dos raios de Prüfer apresente outro comportamento assintótico; mostraremos, inclusive, que para cada raio de Prüfer $R_{n}^{j}$ que se comporte como (3.1.29), podemos associar uma solução subordinada da equação de Schrödinger (3.4) que decai com a mesma taxa de crescimento de $R_{n}^{j}$ (Proposição 4.1.1).

O resultado anterior, juntamente com o Teorema F.3.3 e com uma adaptação do Teorema F.2.17 para operadores esparsos, nos permite uma caracterização precisa do 
espectro das matrizes de Jacobi $J_{\delta, \phi}^{j}$. Antes de seguirmos nessa direção, discutamos a questão da distribuição uniforme dos ângulos de Prüfer.

Consideremos, em primeiro lugar, o modelo determinístico (1.4) estudado por Marchetti et. al.. Apesar de não existir demonstração alguma da uniformidade dos ângulos de Prüfer (que também satisfazem a relação de recorrência (3.1.20), com $a_{k}$ no lugar de $a_{k}^{\omega}=a_{k}+\omega_{k}$ ), é possível mostrar (vide Seção 5 de [MWGA]) que o erro $E$ cometido ao se substituir a soma (3.1.22) pela mesma soma envolvendo a seqüência $\left(\zeta_{k}(\varphi)\right)_{k \geq 1}$ (definida como uma interpolação linear dos ângulos de Prüfer; vide a equação 5.13 de [MWGA]) pode ser tão pequeno quanto se deseje, desde que o parâmetro de esparsidade $\beta>\beta_{0}(E, \lambda, \delta)$ seja suficientemente grande. O problema é que quando fixamos os parâmetros $(E, \lambda, \delta)$, a condição $\beta>\beta_{0}$ torna o suporte essencial da parte puramente pontual do espectro um conjunto vazio. Dessa forma, somente a parte singular-contínua do espectro é assegurada (vide o Teorema 3.3.3 e o Teorema 5.8 de [MWGA] para maiores detalhes).

Neste sentido, a proposta de se inserir uma incerteza na determinação da posição das barreiras, apresentada por Zlatoš em [Z], nos possibilita mostrar que a seqüência $\left(\theta_{n}^{j}\right)_{n \geq 1}$ é u. d. $\bmod \pi$ para todo $\varphi_{j} \in[0, \pi) \backslash A_{j}, A_{j}$ um conjunto de medida de Lebesgue nula (o que confirma a Hipótese 3.1.6), sendo portanto desnecessária a interpolação proposta em [MWGA].

\subsection{Distribuição uniforme dos ângulos de Prüfer}

Demonstramos nesta seção que a seqüência $\left(\theta_{n}^{\omega, j}\right)_{n \geq 1}$ dos ângulos de Prüfer expressos por (3.1.20) é u.d. $\bmod \pi$ para quase todo $\omega \in \Gamma$ e para todo $\varphi_{j} \in[0, \pi) \backslash(\mathbb{Q} \pi \cap[0, \pi))$; em outras palavras, demonstramos a validade da Hipótese 3.1.6, identificando $A_{j}$ com o conjunto dos múltiplos racionais de $\pi$ pertencentes ao intervalo $[0, \pi)$ (obviamente um conjunto com medida de Lebesgue nula; os resultados apresentados independem de $j=$ $0,1, \ldots, L-1$, de modo que omitimos este índice). Para tanto, necessitamos de alguns resultados preliminares, a começar, pelo

Teorema 3.2.1 (Critério de Weyl; Teorema 2.1 de [KN]) A seqüência de números reais $\left(x_{n}\right)_{n \geq 1}$ é u.d. $\bmod \pi$ se, e somente se,

$$
\lim _{N \rightarrow \infty} \frac{1}{N} \sum_{n=1}^{N} e^{2 \pi i h x_{n}}=0,
$$

para qualquer inteiro $h \neq 0$.

Demonstração. A necessidade segue do Corolário 1.2 do Capítulo 1 de [KN]. Suponha agora que a seqüência $\left(x_{n}\right)$ é tal que (3.2.1) é satisfeita. Devemos então mostrar que a relação

$$
\lim _{N \rightarrow \infty} \frac{1}{N} \sum_{n=1}^{N} f\left(x_{n}\right)=\frac{1}{\pi} \int_{0}^{\pi} f(x) d x
$$


é válida para toda função a valores complexos $f$ de período $\pi$. Seja $\varepsilon$ um número positivo arbitrário. Pelo Teorema de aproximação de Weierstrass, existe um polinômio trigonométrico $\Psi(x)$, isto é, uma combinação linear finita de funções do tipo $e^{2 \pi i h x}, h \in \mathbb{Z}$, com coeficientes complexos, tal que

$$
\sup _{0 \leq x \leq \pi}|f(x)-\Psi(x)| \leq \varepsilon .
$$

Temos agora

$$
\begin{aligned}
\left|\frac{1}{\pi} \int_{0}^{\pi} f(x) d x-\frac{1}{N} \sum_{n=1}^{N} f\left(x_{n}\right)\right| & \leq\left|\frac{1}{\pi} \int_{0}^{\pi}(f(x)-\Psi(x)) d x\right| \\
& +\left|\frac{1}{\pi} \int_{0}^{\pi} \Psi(x) d x-\frac{1}{N} \sum_{n=1}^{N} \Psi\left(x_{n}\right)\right| \\
& +\left|\frac{1}{N} \sum_{n=1}^{N}\left(f\left(x_{n}\right)-\Psi\left(x_{n}\right)\right)\right| .
\end{aligned}
$$

O primeiro e o terceiro termos do membro direito são menores ou iguais a $\varepsilon$ independentemente de $N$, graças à (3.2.2). Para $N$ suficientemente grande, o segundo termo do membro direito é menor ou igual a $\varepsilon$ por conta de (3.2.1). Isso encerra a demonstração do teorema.

O outro resultado é o seguinte teorema métrico geral:

Teorema 3.2.2 Seja $\left(u_{n}(x)\right)_{n \geq 1}$ uma seqüência de variáveis aleatórias reais definidas em um espaço de probabilidade $(\Xi, \mathcal{B}, \mu)$. Dados inteiros $h \neq 0, N \geq 1$ e $A \subset \Xi$, definimos

$$
S_{h}(N, x)=\frac{1}{N} \sum_{n=1}^{N} e^{2 i h u_{n}(x)}
$$

$e$

$$
I_{h}(N, A)=\int_{A}\left|S_{h}(N, x)\right|^{2} d \mu(x) .
$$

Se a série $\sum_{N=1}^{\infty} I_{h}(N, A) / N$ converge para cada $h \neq 0$, então a seqüência $\left(u_{n}(x)\right)_{n \geq 1}$ é u.d. $\bmod \pi$ para quase todo $x \in A$ com respeito a $\mu$.

Observação 3.2.3 O Teorema 3.2.2 é uma extensão elementar do Teorema 4.2 de [KN] (extraído de $[D E L])$ ao espaço mensurável $([a, b], \mathcal{B})$, com $\kappa$ a medida de Lebesgue em $\mathbb{R}$.

Demonstração. Necessitamos, antes de mais nada, do seguinte

Teorema 3.2.4 (Teorema de Dini) Seja $\sum_{n=1}^{\infty} c_{n}$ uma série convergente tal que $c_{n} \geq 0$ para todo n. Definamos

$$
r_{n-1} \equiv \sum_{m=n}^{\infty} c_{m}
$$


$n=1,2, \ldots$ Então a série numérica

$$
\sum_{n=1}^{\infty} \frac{c_{n}}{r_{n-1}^{\alpha}}
$$

converge se $\alpha<1$.

Demonstração. Seja $p \geq 2 \in \mathbb{N}$ tal que $\alpha<1-\frac{1}{p}$. Segue da convergência da série $\sum_{n=1}^{\infty} c_{n}$ e da definição (3.2.5) que $r_{n} \rightarrow 0$ no limite $n \rightarrow \infty$, e portanto $r_{n}<1$ para $n>n_{1}$. Dessa forma, é suficiente demonstrarmos a convergência da série numérica

$$
\sum_{n=1}^{\infty} \frac{c_{n}}{r_{n-1}^{1-\tau}}=\sum_{n=1}^{\infty} \frac{r_{n-1}-r_{n}}{r_{n-1}} r_{n-1}^{\tau}
$$

onde $\tau \equiv 1 / p$.

Uma vez que $r_{n} \rightarrow 0$ monotonicamente e $\sum_{n=1}^{\infty}\left(r_{n-1}^{\tau}-r_{n}^{\tau}\right)$ é uma série convergente com termos positivos, é suficiente demonstrar que

$$
\frac{r_{n-1}-r_{n}}{r_{n-1}} r_{n-1}^{\tau} \leq \frac{r_{n-1}^{\tau}-r_{n}^{\tau}}{\tau}
$$

Seja $y \equiv\left(r_{n} / r_{n-1}\right)^{1 / p}$; claramente $0<y \leq 1$. Logo, (3.2.7) é equivalente a $1-y^{p} \leq$ $p(1-y)$, desigualdade que segue da concavidade da função $f(y)=y^{p}$ no intervalo [0,1] e do fato de $z=p(1-y)$ ser a reta tangente à curva $z=1-y^{p}$ no ponto $(y, z)=(1,0)$.

Partamos à demonstração do Teorema 3.2.2. Como mantemos $h$ fixo, deixamos de referenciá-lo no decorrer da demonstração. Uma vez que a série $\sum_{N=1}^{\infty} I(N, A) / N$ converge e é constituída unicamente por termos não-nulos, podemos aplicar o Teorema 3.2.4 sobre esta e definir uma seqüência crescente $(\lambda(N))_{N \geq 1}$ de números reais maiores que $1(\lambda(N) \equiv$ $\left.r_{n-1}^{-\alpha}\right)$, com $\lambda(N) \rightarrow \infty$, tal que $\sum_{N=1}^{\infty} I(N, A) \lambda(N) / N$ ainda converge. Sejam $M_{1}<M_{2}<$ ... inteiros positivos tais que ${ }^{\dagger}$

$$
M_{r+1}=\left[\frac{\lambda\left(M_{r}\right)}{\lambda\left(M_{r}\right)-1} M_{r}\right]+1
$$

para $r \geq 1$. Seja $N_{r}$ o número inteiro pertencente ao intervalo $\left(M_{r}, M_{r+1}\right]$ para o qual $I(N, A)$ assume seu valor mínimo. Então,

$$
I\left(N_{r}, A\right) \leq \frac{1}{M_{r+1}-M_{r}} \sum_{N=M_{r}+1}^{M_{r+1}} I(N, A) \leq \frac{M_{r+1}}{M_{r+1}-M_{r}} \sum_{N=M_{r}+1}^{M_{r+1}} \frac{I(N, A)}{N} .
$$

Como, de acordo com (3.2.8),

$$
M_{r+1}>\frac{\lambda\left(M_{r}\right)}{\lambda\left(M_{r}\right)-1} M_{r}
$$

$\dagger[\cdot]$ representa mais uma vez a parte inteira de um número real. 
segue imediatamente que

$$
\frac{M_{r+1}}{M_{r+1}-M_{r}}<\lambda\left(M_{r}\right)
$$

de onde obtemos

$$
I\left(N_{r}, A\right) \leq \sum_{N=M_{r}+1}^{M_{r+1}} \frac{I(N, A) \lambda(N)}{N}
$$

e assim concluímos que $\sum_{r=1}^{\infty} I\left(N_{r}, A\right)<\infty$. Segue da definição (3.2.4) e do Lema de Fatou (Lema 1.28 de $[\mathrm{Ru}]$ ) que $\sum_{r=1}^{\infty}\left|S\left(N_{r}, x\right)\right|^{2}<\infty$ para quase todo $x \in A$ com respeito a $\mu$ (isto é, a menos de um conjunto de $\mu$-medida nula em $A$ ); conseqüentemente, $\lim _{r \rightarrow \infty} S\left(N_{r}, x\right)=0$ para quase todo $x \in A$. Fica claro de (3.2.8) que $\lim _{r \rightarrow \infty} M_{r+1} / M_{r}=$ 1 , e assim, $\lim _{r \rightarrow \infty} N_{r+1} / N_{r}=1$. Agora, se $N_{r}<N<N_{r+1}$, então

$$
|S(N, x)| \leq\left|S\left(N_{r}, x\right)\right|+\frac{N_{r+1}-N_{r}}{N_{r}}
$$

de onde concluímos que $\lim _{N \rightarrow \infty} S(N, x)=0$ para quase todo $x \in A$, o conjunto $A$ dependendo do inteiro $h$ escolhido no princípio. Se denotarmos por $B$ o conjunto formado pela união contável de todos os conjuntos excepcionais correspondentes a $h= \pm 1, \pm 2, \ldots$, obtemos do critério de Weyl (Teorema 3.2.1) que a seqüência $\left(u_{n}(x)\right)_{n \geq 1}$ é u.d. $\bmod \pi$ para todo $x \in A \backslash B, \operatorname{com} \mu(B)=0$.

Voltemos ao nosso problema. As variáveis aleatórias $\left\{\omega_{j}, j \geq 1\right\}$, definidas no espaço de probabilidade $(\Xi, \mathcal{B}, \mu)$, são consideradas estatisticamente independentes e uniformemente distribuídas em $\Gamma_{j}=\{-j, \ldots, j\}$. Seja $\Gamma=\underset{j=1}{x}\{-j, \ldots, j\}$ o espaço de configuração; denotemos por $\omega=\left(\omega_{1}, \omega_{2}, \ldots\right)$ um elemento do espaço produto $\Gamma$. A medida $\mu$ induz no espaço mensurável $(\Gamma, \mathcal{F})$, onde $\mathcal{F}$ é a $\sigma$-álgebra produto, uma medida produto $\nu(B)=\prod_{j=1}^{\infty} \nu_{j}\left(B_{j}\right)=\mu\left(\omega^{-1}(B)\right), \omega^{-1}(B)=\bigcap_{j \geq 1} \omega_{j}^{-1}\left(B_{j}\right)$, definida para todos os subconjuntos cilíndricos de $\Gamma=\underset{j=1}{\stackrel{\infty}{\infty}}\{-j, \ldots, j\}$, com $\nu_{j}$ a medida uniforme em $\Gamma_{j}$ : $\nu_{j}(k)=1 /(2 j+1)$ para todo $k \in\{-j, \ldots, j\}$. Também denotamos a seqüência de ângulos de Prüfer, definida pela relação de recorrência (3.1.20), ou por $\left(\theta_{k}(x)\right)_{k \geq 1}$ ou por $\left(\theta_{k}^{\omega}\right)_{k \geq 1}$, dependendo se levamos em consideração o espaço de probabilidade $(\Xi, \mathcal{B}, \mu)$ ou o espaço $(\Gamma, \mathcal{F}, \nu)$.

Apresentamos a seguir o

Teorema 3.2.5 (Teorema 2.2 de [CMW1]) A seqüência $\left(\theta_{k}(x)\right)_{k \geq 1}\left(\left(\theta_{k}^{\omega}\right)_{k \geq 1}\right)$, dos ângulos de Prüfer é u.d. $\bmod \pi$ para todo $\varphi \in[0, \pi) \backslash(\mathbb{Q} \pi \cap[0, \pi])$ e todo $x \in \Xi(\omega \in \Gamma)$ a menos de um conjunto $B$ com medida $\mu(\nu)$ nula (presente na demonstração do Teorema 3.2.2). 
Demonstração. Devemos, de acordo com o Teorema 3.2.2, mostrar que $\sum_{N=1}^{\infty} \frac{I_{h}(N, \Gamma)}{N}<$ $\infty, I_{h}(N, \Gamma)$ definida por (3.2.4). Basta, contudo, demonstramos sua convergência absoluta. Temos de (3.2.3)

$$
\begin{aligned}
I_{h}(N, \Gamma) & =\int_{\Gamma}\left|S_{h}(N, x)\right|^{2} d \mu(x) \\
& \leq \frac{1}{N}+\frac{2}{N^{2}} \sum_{1 \leq m<n \leq N}\left|\int_{\Gamma} e^{2 i h\left(\theta_{m}(x)-\theta_{n}(x)\right)} d \mu(x)\right| .
\end{aligned}
$$

Uma vez que $\theta_{m}^{\omega}$, com $m<n$, e $\widetilde{\theta}_{n}^{\omega}$, dado por $\theta_{n}^{\omega}=g\left(\theta_{n-1}^{\omega}, \varphi\right)-\left(\beta^{n}+\omega_{n}-\omega_{n-1}\right) \varphi \equiv$ $\tilde{\theta}_{n}^{\omega}-\omega_{n} \varphi$, são estatisticamente independentes de $\omega_{n}$, obtemos

$$
\begin{aligned}
\left|\int_{\Xi} e^{2 i h\left(\theta_{m}(x)-\theta_{n}(x)\right)} d \mu(x)\right| & =\left|\int_{\Gamma \backslash \Gamma_{n}} e^{2 i h\left(\theta_{m}^{\omega}-\tilde{\theta}_{n}^{\omega}\right)} d \nu(\omega)\right|\left|\int_{\Gamma_{n}} e^{2 i h \omega_{n} \varphi} d \nu_{n}\left(\omega_{n}\right)\right| \\
& \leq\left|\int_{\Gamma_{n}} e^{2 i h \omega_{n} \varphi} d \nu_{n}\left(\omega_{n}\right)\right|
\end{aligned}
$$

O membro direito de (3.2.10), a função característica de $\nu_{n}$ em $2 h \varphi$, pode ser computado explicitamente:

$$
\begin{aligned}
\left|\frac{1}{2 n+1} \sum_{k=-n}^{n} e^{2 i h k \varphi}\right| & =\left|\frac{1}{2 n+1} \frac{\sin (2 n+1) h \varphi}{\sin h \varphi}\right| \\
& \leq \frac{1}{(2 n+1)|\sin h \varphi|}<\infty
\end{aligned}
$$

desde que $\varphi \notin \mathbb{Q} \pi \cap[0, \pi)$, justificando portanto a restrição imposta pelo teorema.

Temos de (3.2.9), (3.2.10) e (3.2.11)

$$
I_{h}(N, \Gamma) \leq \frac{1}{N}+\frac{2}{|\sin h \varphi| N^{2}} \sum_{1 \leq m<n \leq N} \frac{1}{2 n+1}<\frac{1}{N}\left(1+\frac{1}{|\sin h \varphi|}\right),
$$

e portanto $\sum_{N=1}^{\infty} I_{h}(N, \Gamma) / N$ é finito para todo $h \neq 0$. Concluímos a demonstração evocando o Teorema 3.2.2.

Observação 3.2.6 1. Um resultado análogo a este foi obtido por Zlatoš (Seção 6 de $[Z])$. No entanto, acreditamos que as técnicas empregadas anteriormente, além de mais simples, são mais gerais: Zlatoš exclui do seu resultado todo $\lambda=2 \cos \varphi \in$ $[-2,2]$ tal que $\operatorname{dist}(q \lambda, \mathbb{Z}) \leq 1 / q^{2}$ para $q$ suficientemente grande (condição Diophantina); como acabamos de ver, basta excluirmos o conjunto $\cos \mathbb{Q} \pi$.

2. Se $\varphi$ é um número racional, existe um $h \in \mathbb{Z}, h \neq 0$, tal que $h \varphi=m \in \mathbb{Z}$. De (3.2.11)

$$
\frac{\sin (2 n+1) h \varphi}{\sin h \varphi}=\lim _{h \rightarrow m / \varphi} \frac{\sin (2 n+1) h \varphi}{\sin h \varphi}=2 n+1
$$


o que implica em $I_{h}(N, \Xi) \leq O(1)$. Conseqüentemente, as estimativas da soma $\sum_{N=1}^{\infty} I_{h}(N, \Gamma) / N$ empregadas na demonstração do Teorema 3.2.5 divergem.

3. A hipótese sobre a uniformidade de $\nu_{j}$ não é necessária para nossa análise, no entanto nós a assumimos por uma questão de simplicidade. É suficiente definir as variáveis aleatórias $\omega_{j}$ como suportadas em um intervalo $\Gamma_{j} \equiv\left[-j^{\varepsilon}, j^{\varepsilon}\right] \cap \mathbb{Z}, \varepsilon$ qualquer real positivo, a fim de assegurar que os ângulos de Prüfer $\left(\theta_{k}^{\omega}\right)_{k \geq 1}$ sejam u. d. $\bmod \pi$. Fica claro, observando a demonstração do Teorema 3.2.5, que teríamos nesse caso

$$
\sum_{N=1}^{\infty} \frac{I_{h}(N, \Gamma)}{N} \leq \sum_{N=1}^{\infty} \frac{O(1)}{N^{1+\varepsilon}}<\infty
$$

o que, pelo Teorema 3.2.2, demonstra nossa afirmação.

\subsection{Demonstração da existência de transição espec- tral}

Os resultados da Seção 3.2 nos mostram a validade da Hipótese 3.1.6 para a seqüência $\left(\theta_{n}^{j}\right)_{n \geq 1}$ de ângulos de Prüfer definidos pela relação de recorrência (3.1.20), o que por sua vez garante a estimativa (3.1.29).

Necessitamos de mais um ingrediente para determinar completamente os tipos espectrais de cada matriz de Jacobi $J_{\delta, \phi}^{j}$.

Teorema 3.3.1 (Lema 3.6 de [CMW1]) Sejam $J_{P, \phi}^{j}$ as matrizes de Jacobi associadas aos operadores $\Delta_{P, \phi}^{j}, j \in\{0, \ldots, L-1\}$, definidos por $(3.2)$, com $P \in \mathcal{P}_{\delta, \beta}$ e $\phi$ a condição de contorno (1.5). Denotemos por $t_{n}^{j}=\left\|T\left(a_{n}+1 ; \lambda_{j}\right)\right\|$ a norma espectral associada ao produto das $a_{n}+1$ primeiras matrizes de transferência (3.5) para algum $\lambda_{j} \equiv \lambda-$ $2 \cos (2 \pi j / L) \in[-2,2] . S e$

$$
\sum_{n=1}^{\infty} \beta^{n}\left(t_{n}^{j}\right)^{-2}<\infty
$$

então existe uma solução fortemente subordinada (vide a Definição F.2.19), $u_{\infty}^{j}$, de (3.4) que obedece a estimativa

$$
\left\|T\left(a_{n}+1 ; \lambda_{j}\right) u_{\infty}^{j}\right\|^{2} \leq\left(t_{n}^{j}\right)^{-2}+\left(\frac{\pi}{2}\right)^{2} B_{j}^{4}\left(t_{n}^{j}\right)^{2}\left(\sum_{m=n}^{\infty}\left(t_{n}^{j}\right)^{-2}\right)^{2},
$$

onde $B_{j} \equiv \sqrt{\frac{1+\left|\cos \varphi_{j}\right|}{1-\left|\cos \varphi_{j}\right|}}\left(1+\frac{1+\lambda_{j}^{2}}{\left(1-\delta^{2}\right)}\right)$. Em particular, se

$$
\sum_{n=1}^{\infty} \beta^{n}\left(t_{n}^{j}\right)^{2}\left(\sum_{m=n}^{\infty}\left(t_{m}^{j}\right)^{-2}\right)^{2}<\infty
$$


então, para cada $j$, a equação de Schrödinger (3.4) apresenta uma solução $l^{2}\left(\mathbb{Z}_{+}, \mathbb{C}\right)$.

Observação 3.3.2 1. O Teorema 3.3.1 nada mais é do que uma otimização do Teorema F.2.15 para matrizes de Jacobi esparsas; estendemos sua demonstração a fim de facilitar sua compreensão.

2. Evitamos explicitar o índice $j$ em $t_{n}$ a fim de não sobrecarregar a notação. Os resultados seguintes independem de $j$.

Demostração. Começamos adaptando o Teorema F.2.12 para o modelo considerado em nosso trabalho.

De (3.5) e (1.9),

$$
\left\|T\left(k, k-1 ; \lambda_{j}\right)\right\|^{2} \leq\left\|T\left(k, k-1 ; \lambda_{j}\right)\right\|_{E}^{2} \leq 1+\frac{1+\lambda_{j}^{2}}{(1-\delta)^{2}}<\infty
$$

caso $\delta \in(0,1)$, onde $\|\cdot\|_{E}$ representa a norma matricial euclideana, com $k, k-1 \in \mathcal{A}^{\omega}$; de outra forma, $T\left(k, k-1 ; \lambda_{j}\right)$ é semelhante a uma rotação horária $R\left(\varphi_{j}\right)$ por $\varphi_{j}=$ $(1 / 2) \arccos (\lambda-2 \cos (2 \pi j / L))$ (vide equação (3.1.4)). Escrevemos

$$
T\left(a_{n}+1 ; \lambda_{j}\right)=A_{n}\left(\lambda_{j}\right) \cdots A_{1}\left(\lambda_{j}\right)
$$

onde, para cada $m \geq 2$,

$$
A_{m}\left(\lambda_{j}\right)=T\left(a_{m}+1, a_{m} ; \lambda_{j}\right) \cdots T\left(a_{m-1}+2, a_{m-1}+1 ; \lambda_{j}\right)=T_{+} T_{-} T_{0}^{\beta^{m}-2},
$$

graças às definições (3.1.1), (3.1.2) e (3.1.3). Designando por $s_{n}=\left\|A_{n}\left(\lambda_{j}\right)\right\|$ a norma espectral de $A_{n}\left(\lambda_{j}\right)$ (novamente omitimos a dependência em $j$ ), obtemos

$$
s_{n} \leq C_{j}\left(1+\frac{1+\lambda_{j}^{2}}{(1-\delta)^{2}}\right) \equiv B_{j},
$$

$\operatorname{com} C_{j}=\sqrt{\left(1+\left|\cos \varphi_{j}\right|\right) /\left(1-\left|\cos \varphi_{j}\right|\right)}$, uniformemente em $n$ (vide a Proposição F.3.2). Como conseqüência,

$$
\sum_{n=1}^{\infty} \frac{s_{n+1}^{2}}{t_{n}^{2}}<\infty
$$

verifica a hipótese do Teorema F.2.15 e portanto garante a existência de uma solução subordinada $v^{j}$ associada a $\lambda$. Uma vez que o parâmetro de esparsidade satisfaz a desigualdade $\beta \geq 2$, a condição (3.3.1) é mais restritiva que a desigualdade (3.3.5), e assim a hipótese do Teorema F.2.12 é automaticamente validada pela hipótese do teorema.

As matrizes de transferência $T_{0}, T_{+-}:=T_{+} T_{-}$dadas respectivamente por (3.1.1), (3.1.2), (3.1.3), são, assim como $T\left(a_{n}+1 ; \lambda_{j}\right)$ e $T^{*}\left(a_{n}+1 ; \lambda_{j}\right)$, matrizes reais unimodulares $2 \times 2$. Logo, o produto $T^{*}\left(a_{n}+1 ; \lambda_{j}\right) T\left(a_{n}+1 ; \lambda_{j}\right)$ é uma matriz real simétrica unimodular 
$2 \times 2$ cujos autovalores são $t_{n}^{2}$ e $t_{n}^{-2}$; a estes se associam os autovetores ortonormais $\mathbf{u}_{n}^{+}$e $\mathbf{u}_{n}^{-}$, respectivamente: $\left(\mathbf{u}_{n}^{+}, \mathbf{u}_{n}^{-}\right)=0$. Fazemos $\mathbf{u}_{\phi}^{j}=\left(\begin{array}{c}\cos \phi^{j} \\ \sin \phi^{j}\end{array}\right)$ e definimos $\phi_{n}^{j}$ por

$$
\mathbf{u}_{\phi_{n}^{j}}=\mathbf{u}_{n}^{-}
$$

Obviamente, $\mathbf{u}_{n}^{+}=\mathbf{u}_{\phi_{n}^{j}+\pi / 2}$ e assim temos do teorema espectral

$$
\begin{aligned}
\left\|T\left(a_{n}+1 ; \lambda_{j}\right) \mathbf{u}_{\phi^{j}}\right\|^{2} & =\left(\mathbf{u}_{\phi^{j}}, T^{*}\left(a_{n}+1 ; \lambda_{j}\right) T\left(a_{n}+1 ; \lambda_{j}\right) \mathbf{u}_{\phi^{j}}\right) \\
& =t_{n}^{2}\left|\left(\mathbf{u}_{\phi^{j}}, \mathbf{u}_{n}^{+}\right)\right|^{2}+t_{n}^{-2}\left|\left(\mathbf{u}_{\phi^{j}}, \mathbf{u}_{n}^{-}\right)\right|^{2} \\
& =t_{n}^{2} \sin ^{2}\left(\phi^{j}-\phi_{n}^{j}\right)+t_{n}^{-2} \cos ^{2}\left(\phi^{j}-\phi_{n}^{j}\right) .
\end{aligned}
$$

Seja o vetor $\mathbf{u}^{j}=\left(u_{k}^{j}\right)_{k \geq 0}$, com $u_{k}^{j}=\left(T\left(k ; \lambda_{j}\right) \mathbf{u}_{\phi^{j}}\right)_{2}$, a solução da equação de Schrödinger (3.4) sujeita à condição de contorno (1.5).

Podemos mostrar, usando propriedades da norma de uma matriz, a relação (3.3.7) tomada no ponto $n+1$, e a definição (3.3.6) (vide a demonstração do Teorema F.2.12), que

$$
\left|\phi_{n}^{j}-\phi_{n+1}^{j}\right| \leq \frac{\pi}{2} \frac{s_{n+1}^{2}}{t_{n}^{2}} .
$$

Concluímos do resultado acima e da condição (3.3.5) que a seqüência $\left(\phi_{n}^{j}\right)_{n \geq 1}$ possui um limite $\phi_{\infty}^{j}=\lim _{n \rightarrow \infty} \phi_{n}^{j}$. Desse modo, a equação (3.3.7) e a estimativa telescópica

$$
\left|\phi_{n}^{j}-\phi_{\infty}^{j}\right| \leq \sum_{m=n}^{\infty}\left|\phi_{m}^{j}-\phi_{m+1}^{j}\right| \leq \frac{\pi}{2} \sum_{m=n}^{\infty} \frac{s_{m+1}^{2}}{t_{m}^{2}}
$$

nos levam a

$$
\begin{aligned}
\left\|T\left(a_{n}+1 ; \lambda_{j}\right) \mathbf{u}_{\phi_{\infty}^{j}}\right\|^{2} & \leq t_{n}^{2}\left(\phi_{\infty}^{j}-\phi_{n}^{j}\right)^{2}+t_{n}^{-2} \\
& \leq\left(\frac{\pi}{2}\right)^{2} B_{j}^{4} t_{n}^{2}\left(\sum_{m=n}^{\infty} t_{m}^{-2}\right)^{2}+t_{n}^{-2},
\end{aligned}
$$

exatamente (3.3.2). Repare que, pela definição (F.1.2) da matriz de transferência, $u_{k}^{j}=$ $\left(T\left(k ; \lambda_{j}\right) \mathbf{u}_{\phi_{\infty}^{j}}\right)_{2}$ é uma solução fortemente subordinada de (3.4) no sentido que, para $v_{k}^{j} \equiv\left(T\left(k ; \lambda_{j}\right) \mathbf{u}_{\phi_{\infty}^{j}+\pi / 2}\right)_{2}$, temos

$$
\lim _{k \rightarrow \infty} \frac{\left|\left(u_{k}^{j}\right)^{2}+\left(u_{k+1}^{j}\right)^{2}\right|}{\left|\left(v_{k}^{j}\right)^{2}+\left(v_{k+1}^{j}\right)^{2}\right|}=0
$$

já que $\left\|T\left(a_{n}+1 ; \lambda_{j}\right) \mathbf{u}_{\phi_{\infty}^{j}+\pi / 2}\right\| \geq t_{n} / 2$ para $n$ suficientemente grande e $\left\|T\left(k ; \lambda_{j}\right) \mathbf{u}_{\phi^{j}}\right\| \leq$ $\left\|T\left(k, a_{n}+1 ; \lambda_{j}\right)\right\|\left\|T\left(a_{n}+1 ; \lambda_{j}\right) \mathbf{u}_{\phi^{j}}\right\| \leq B_{j}\left\|T\left(a_{n}+1 ; \lambda_{j}\right) \mathbf{u}_{\phi^{j}}\right\|$, para algum $n$ tal que $a_{n}+$ $1 \leq k<a_{n+1}$. 
Resta-nos mostrar que a solução subordinada também pertence a $l^{2}\left(\mathbb{Z}_{+}, \mathbb{C}\right)$ caso satisfaça as hipóteses (3.3.1) e (3.3.3). Todavia, é suficiente verificá-lo (graças à Proposição F.3.2, que demonstra a equivalência entre as normas $\|\cdot\|$ e $\|\cdot\|_{U}$ ) para a norma $U_{j}$, definida por (F.3.6), com $U_{j}$ dado por (3.1.5). Para qualquer $k \in \mathbb{N}$, escolhemos $n$ tal que a condição $a_{n}+1 \leq k<a_{n+1}$ seja satisfeita. Segue em analogia a (3.1.15), a desigualdade

$$
\left\|T\left(k ; \lambda_{j}\right) \mathbf{u}_{\phi_{\infty}^{j}}\right\|_{U_{j}}^{2}=\left\|T\left(a_{n}+1 ; \lambda_{j}\right) \mathbf{u}_{\phi_{\infty}^{j}}\right\|_{U_{j}}^{2} \leq\left(1+\left|\cos \varphi_{j}\right|\right)\left\|T\left(a_{n}+1 ; \lambda_{j}\right) \mathbf{u}_{\phi_{\infty}^{j}}\right\|^{2}
$$

o que, pela condição de esparsidade (1.11), pela hipótese (3.3.3) e por (3.3.8) resulta em

$$
\sum_{k=1}^{\infty}\left\|T\left(k ; \lambda_{j}\right) \mathbf{v}_{\hat{\phi}^{j}}\right\|_{U_{j}}^{2} \leq B_{j}^{\prime} \sum_{n=1}^{\infty} \beta^{n} t_{n}^{2}\left(\sum_{m=n}^{\infty} t_{m}^{-2}\right)^{2}+B_{j}^{\prime \prime} \sum_{n=1}^{\infty} \beta^{n} t_{n}^{-2}<\infty
$$

para algumas constantes $B_{j}^{\prime}$ e $B_{j}^{\prime \prime}$, concluíndo assim a demonstração do teorema.

Podemos, desse modo, otimizar o Teorema F.2.17 (que relaciona os suportes mínimos das partes absolutamente contínua, etc., da medida $\mu_{j}$ ao comportamento assintótico de $\left\|T\left(n ; \lambda_{j}\right)\right\|$ no limite $\left.n \rightarrow \infty\right)$ para as matrizes de Jacobi esparsas $J_{\delta, \phi}^{j}$; de fato, segue do Teorema 3.3.1 a inclusão

$$
\Sigma_{\mathrm{pp}}^{\prime \prime \prime} \supset\left\{\lambda \in \mathbb{R}: \sum_{n=1}^{\infty} \beta^{n}\left(t_{n}^{j}\right)^{2}\left(\sum_{m=n}^{\infty}\left(t_{m}^{j}\right)^{-2}\right)^{2}<\infty\right\} \bigcap \Sigma_{0}^{C},
$$

onde $\Sigma_{\mathrm{pp}}^{\prime \prime \prime}$ representa o suporte mínimo da parte puramente pontual do espectro como definido no Teorema E.11 e $\Sigma_{0}:=\{\lambda \in \mathbb{R}$ : existe uma solução subordinada da equação de Schrödinger (3.4), porém esta não satisfaz a condição de contorno (1.5)\}. É bastante natural que busquemos adaptar os demais resultados do Teorema F.2.17 para esse tipo de operadores; é justamente esta adaptação que nos permite enunciar o

Teorema 3.3.3 Sejam $J_{\delta, \phi}^{j}$ as matrizes de Jacobi definidas como no Teorema 3.3.1. Sejam os intervalos

$$
I_{j} \equiv\left\{\lambda=\lambda_{j}+2 \cos (2 \pi j / L): \lambda_{j} \in[-2,2] \backslash A, \frac{p^{2}}{\left(1-p^{2}\right)^{2}}(\beta-1)\left(4-\lambda_{j}^{2}\right)>1\right\},
$$

com $p \in(0,1), \beta \in \mathbb{N}, \beta \geq 2$ e $A=2 \cos \mathbb{Q} \pi$ (como na demonstração do Teorema 3.2.5). Então, para quase todo $\omega$ com respeito à medida produto uniforme em $\Gamma=\underset{k=1}{\times}\{-k, \ldots, k\}$,

(a) existe um conjunto $A_{1}^{j}$ com medida de Lebesgue nula tal que o espectro, quando restrito ao conjunto $I_{j} \backslash A_{1}^{j}$, é puramente singular-contínuo;

(b) o espectro de $J_{\delta, \phi}^{j}$ é puramente pontual quando restrito ao conjunto $I_{j}^{c} \equiv[-2+$ $2 \cos (2 \pi j / L), 2+2 \cos (2 \pi j / L)] \backslash I_{j}$ para quase todo $\phi \in[0, \pi)$, em que $\phi$ caracteriza a condição de contorno (1.5). 
Observação 3.3.4 1. O Teorema 3.3.3 aprimora e estende os resultados do Teorema 4.4 de $[M W G A]$ às matrizes de Jacobi $J_{\delta, \phi}^{j}$ : a extensão mencionada se refere ao fato das posições das barreiras serem agora variáveis aleatórias; aprimoramos o resultado ao passo que determinamos precisamente as regiões onde os espectros puramente pontual e singular-contínuo se fazem presentes.

2. A demonstração do Teorema 3.3.3 essencialmente reproduz a demonstração do Teorema 4.4 de [MWGA], exceto por algumas particularidades.

3. A demonstração desenvolvida por Zlatoš para um resultado análogo (vide o Teorema 6.3 de [Z]) está incompleta, já que parece indicar que medidas com dimensão Hausdorff nula sejam discretas. Na Subseção 5.2, apresentamos um exemplo de modelo, com esparsidade super-geométrica, cujo espectro essencial do operador associado é puramente singular-contínuo e cuja medida espectral possui dimensão Hausdorff 0. Esse resultado contradiz a conclusão de Zlatoš de que se obtém a parte puramente pontual do espectro simplesmente excluíndo o conjunto de pontos para os quais a dimensão Hausdorff não é positiva. A fim de que asseguremos a existência de uma solução $l^{2}\left(\mathbb{Z}_{+}, \mathbb{C}\right.$ ) da equação de Schrödinger (3.4) (o que implica existência de espectro puramente pontual), as condições (3.3.1) e (3.3.3) devem ser satisfeitas simultaneamente: apesar do conjunto $I_{j}$ dos parâmetros $\lambda$ que obedecem (3.3.11) excluir o espectro puramente pontual (ele é complementar ao conjunto dos parâmetros para os quais a condição (3.3.1) é satisfeita), necessitamos, para o caso de uma esparsidade geométrica, do comportamento assintótico exato de $t_{n}^{2}=\left\|T\left(a_{n} ; \lambda\right)\right\|^{2}$, de modo a garantir a validade da condição (3.3.3).

Demonstração. Devemos mostrar, para $p$ e $\beta$ fixos, a inclusão

$$
I_{j} \backslash A_{1}^{j} \subseteq\left\{\lambda \in \mathbb{R}: \sum_{n=0}^{\infty}\left\|T\left(n ; \lambda_{j}\right)\right\|^{-2}=\infty\right\}
$$

( $A_{1}^{j}$ um conjunto de medida de Lebesgue nula), de modo que o ítem (a) segue diretamente do Teorema F.2.17. Da mesma maneira, se

$$
\left[I_{j} \backslash A_{1}^{j}\right]^{C} \subseteq\left\{\lambda \in \mathbb{R}: \sum_{n=1}^{\infty} \beta^{n}\left(t_{n}^{j}\right)^{2}\left(\sum_{m=n}^{\infty}\left(t_{m}^{j}\right)^{-2}\right)^{2}<\infty\right\}
$$

então o ítem (b) segue do Teorema 3.3.1 e da Proposição 3.3.5.

Definimos $n$ tal que a relação $a_{N}^{\omega} \leq n<a_{N+1}^{\omega}$ seja satisfeita para algum $N \in \mathbb{N}$. Segue da condição de esparsidade (1.11), da definição (F.3.6) e da relação (3.1.4),

$$
\sum_{m \leq n}\left\|T\left(m ; \lambda_{j}\right)\right\|_{U_{j}}^{-2}=\sum_{k \leq N+1}\left\|T\left(a_{k}^{\omega} ; \lambda_{j}\right)\right\|_{U_{j}}^{-2} \beta^{k+1}+\left\|T\left(a_{N}^{\omega} ; \lambda_{j}\right)\right\|_{U_{j}}^{-2} \sum_{n \leq m<a_{N+1}^{\omega}} 1 .
$$


Obtemos diretamente da Proposição F.3.2 e do Teorema F.3.3 as desigualdades

$$
\left\|T\left(m ; \lambda_{j}\right)\right\|^{-2} \leq C_{j}^{2}\left\|T\left(a_{k}^{\omega} ; \lambda_{j}\right)\right\|_{U_{j}}^{-2} \leq C_{j}^{2}\left(\max _{i \in\{1,2\}} R_{k}^{j}\left(\theta_{i}^{j}\right)\right)^{-2}
$$

$\mathrm{e}$

$$
\left\|T\left(m ; \lambda_{j}\right)\right\|^{-2} \geq C_{j}^{-2}\left\|T\left(a_{k}^{\omega} ; \lambda_{j}\right)\right\|_{U_{j}}^{-2} \geq \tilde{C}_{j}^{-2}\left(\max _{i \in\{1,2\}} R_{k}^{j}\left(\theta_{i}^{j}\right)\right)^{-2}
$$

para todo $a_{k}^{\omega} \leq m<a_{k+1}^{\omega}, \operatorname{com} C_{j}=\sqrt{\left(1+\left|\cos \varphi_{j}\right|\right) /\left(1-\left|\cos \varphi_{j}\right|\right)}$ e $\tilde{C}_{j}=C_{j} / \mid \sin \left(\theta_{1}^{j}-\right.$ $\left.\theta_{2}^{j}\right) / 2 \mid$, onde $\theta_{1}^{j}, \theta_{2}^{j} \in[0, \pi)$ representam um par de ângulos de Prüfer iniciais tais que $0<\left|\theta_{1}^{j}-\theta_{2}^{j}\right|<\pi / 2$ (vide o Lema F.3.5).

Definamos

$$
S_{N, M}^{ \pm}\left(\beta, \varphi_{j}\right) \equiv \sum_{k=N}^{M} C_{k, j}^{ \pm}\left(\beta^{k+1} \pm 2 k\right) r_{j}^{-k}
$$

(o termo $2 k$ se deve à incerteza associada à posição da $k$-ésima barreira: $\beta^{k+1}-2 k \leq$ $a_{k}^{\omega} \leq \beta^{k+1}+2 k-1$ para todo $\left.\omega \in \Gamma\right)$, onde $r_{j}$ é definido por (3.1.30).

Obtemos diretamente do Lema 3.1.11, de (3.3.12), (3.3.14) e da definição (3.3.15), as desigualdades

$$
\tilde{C}_{j}^{-2} S_{N+1, M}^{-} \leq \sum_{m=n}^{a_{M}+1}\left\|T\left(m ; \lambda_{j}\right)\right\|^{-2} \leq C_{j}^{2} S_{N, M}^{+}
$$

válidas para todos inteiros $M \geq N+1$ e $\lambda \in B_{j}, \operatorname{com}^{\dagger}$

$$
B_{j} \equiv 2 \cos ([0, \pi] \backslash \mathbb{Q} \pi)+2 \cos (2 \pi j / L)
$$

onde $2 \cos (\mathbb{Q} \pi \cap[0, \pi))$ representa o conjunto de "energias" onde a seqüência $\left(\theta_{n}^{\omega, j}\right)_{n \geq 0}$ não é uniformemente distribuída módulo $\pi$ (vide o Teorema 3.2.5). Fica claro do Lema 3.1.11, de (3.1.30), (3.3.16) e (3.3.17) que

$$
B_{j} \subseteq\left\{\lambda: \lim _{n \rightarrow \infty}\left\|T\left(n ; \lambda_{j}\right)\right\|=\infty\right\},
$$

ou seja, $B_{j}$ pertence ao complemento de $\Sigma_{\text {ac }}$ pelo Teorema F.2.17. Dessa forma, exceto pelo conjunto $A_{j}^{1}=(\mathbb{Q} \pi \cap[0, \pi)) \cup A_{j}^{*}$, de medida de Lebesgue nula ( $A_{j}^{*}$ é um conjunto de medida de Lebesgue nula associada à definição do suporte essencial $\Sigma_{\mathrm{ac}}$ da medida espectral $\mu_{j}$ ), o conjunto

$$
B_{j}^{1} \equiv 2 \cos \left([0, \pi] \backslash A_{j}^{1}\right)+2 \cos (2 \pi j / L)
$$

\footnotetext{
${ }^{\dagger}$ Aqui, $f(A)$ representa o conjunto $\{f(x): x \in A\}$.
} 
pertence ao espectro singular. Da definição (3.3.15) e do membro esquerdo de (3.3.16) concluímos, no limite $M \rightarrow \infty$, que

$$
\sum_{n=0}^{\infty}\left\|T\left(n ; \lambda_{j}\right)\right\|^{-2}=\infty
$$

segue caso a desigualdade

$$
4 p^{2}(\beta-1)>\left(1-p^{2}\right)^{2} \operatorname{cosec}^{2} \varphi_{j}
$$

seja satisfeita. É simples verificar que (3.3.19) define o conjunto $I_{j}$ dado por (3.3.11); desse modo, concluímos de (3.3.18) e do Teorema F.2.17 que o espectro essencial da matriz de Jacobi $J_{\delta, \phi}^{j}$ é puramente singular-contínuo quando restrito ao conjunto $I_{j} \backslash\left(A_{j}^{1} \cap I_{j}\right)$, o que demonstra a parte (a) do teorema.

Necessitamos, para a parte (b) do Teorema, da

Proposição 3.3.5 (Proposição 4.2 de [MWGA]) Suponha que a inclusão

$$
[a, b] \subset \sigma\left(J_{\delta, \phi}\right)
$$

$J_{\delta, \phi}$ a matriz de Jacobi associada ao operador $\Delta_{\delta, \phi}$, seja válida para todo $\phi \in[0, \pi)$, e que exista um subconjunto $A \subset[a, b]$ de medida de Lebesgue nula independente de $\phi$ tal que, se $\lambda \in[a, b] \backslash A$, então a equação (3.4) possui uma solução $u \in l^{2}\left(\mathbb{Z}_{+}, \mathbb{C}\right)$ independentemente da condição inicial $u_{-1}$. Então $\sigma\left(J_{\delta, \phi}\right)$ possui apenas espectro puramente pontual no intervalo $[a, b]$ para quase toda condição de contorno $\phi$ (com respeito à medida de Lebesgue) dada por (1.5).

Demonstração. Seja $\lambda \in[a, b] \backslash A$. Segue da hipótese da proposição que (3.4) possui uma solução $l^{2}\left(\mathbb{Z}_{+}, \mathbb{C}\right)$. Logo, pelo Teorema II.3 de [Si], duas alternativas são possíveis:

1. $\lambda$ é solução da equação de Schrödinger (3.4) sujeita à condição de contorno de Dirichlet (D.1.9),

2. $G(z)=\int \frac{d \mu(y)}{(\lambda-y)^{2}}<\infty$.

A primeira alternativa segue no máximo para um conjunto contável de pontos; no entanto, por hipótese, o conjunto de pontos para os quais a equação (3.4) apresenta solução possui medida de Lebesgue positiva, ou seja, é o complemento de um conjunto de medida de Lebesgue nula. Assim, mesmo excluíndo um conjunto contável que satisfaça a primeira alternativa, concluímos que $G(\lambda)<\infty$ para quase todo $\lambda \in[a, b]$, o que, pelo critério de Simon-Wolff (Teorema II.5 de [Si]), implica que a matriz de Jacobi $J_{\delta, \phi}$ possui apenas espectro puramente pontual nesse intervalo, para quase toda condição de contorno $\phi$. Finalmente, a inclusão dos $\lambda$ que satisfazem a primeira alternativa não altera o resultado da proposição. 
Provemos agora a parte (b) do teorema, assumindo que

$$
4 p^{2}(\beta-1)<\left(1-p^{2}\right)^{2} \operatorname{cosec}^{2} \varphi_{j} .
$$

Aqui surge a diferença fundamental entre o Teorema 3.3.3 e o Teorema 4.4 de [MWGA]: usamos o resultado do Teorema 3.3.1, com ligeiras modificações ao caso aleatório, em substituição à desigualdade (F.2.30). A saber, se definirmos

$$
S=\sum_{m=n} C_{m, j} r_{j}^{-m}
$$

temos novamente do Lema 3.1.11, e das relações (3.3.12) e (3.3.13),

$$
\sum_{m=n}^{\infty}\left\|T\left(a_{m}+1 ; \lambda_{j}\right)\right\|^{-2} \leq C_{j}^{2} S .
$$

Por fim, segue novamente do Lema 3.1 .11 e das relações (3.3.13), (3.3.14), (3.3.22) e (3.3.23) que

$$
\begin{aligned}
\sum_{n=1}^{\infty}\left(\beta^{n}+\omega_{j}-\omega_{j-1}\right)\left\|T\left(a_{n}+1 ; \lambda_{j}\right)\right\|^{2} & \left(\sum_{m=n}^{\infty}\left\|T\left(a_{m}+1 ; \lambda_{j}\right)\right\|^{-2}\right)^{2} \\
& \leq C_{j}^{\prime} \sum_{n=1}^{\infty}\left(\beta^{n}+2 n\right) C_{n, j} r_{j}^{n} S^{2} \\
\leq & C_{j}^{\prime \prime} \sum_{n=1}^{\infty}\left(\beta^{n}+2 n\right)\left(C_{n, j}\right)^{3} r_{j}^{-n}
\end{aligned}
$$

converge para $\lambda$ tal que (3.3.21) é satisfeita. Segue do Lema 3.3.1 e da desigualdade acima que se $\lambda$ pertence ao conjunto

$$
B_{j}^{2}=I_{j}^{c} \backslash\left(A_{j}^{1} \cap I_{j}^{c}\right),
$$

( $I_{j}^{c}$ o conjunto complementar de $I_{j}$ em $\left.[-2+2 \cos (2 \pi j / L), 2+2 \cos (2 \pi j / L)]\right)$, então a equação de Schrödinger $\left(J_{\delta, \phi}^{j}-\lambda I\right) u=0$ (vide a Observação 3.1) possui uma solução $l^{2}\left(\mathbb{Z}_{+}, \mathbb{C}\right)$. Uma vez que, pelo Teorema 2.4.5, $I_{j}^{c} \in \sigma_{\text {ess }}\left(J_{\delta, \phi}^{j}\right)$ para todo $\phi \in[0, \pi)$, as hipóteses da Proposição 3.3.5 são satisfeitas e $J_{\delta, \phi}^{j}$ possui espectro unicamente puramente pontual em $I_{j}^{c}$ para quase todo $\phi \in[0, \pi)$, o que conclui a demonstração da parte (b) do teorema.

O Teorema 3.3.3 nos mostra que além de existir uma transição entre as partes singularcontínua e puramente pontual do espectro, esta é aguda, ou seja, não existe uma região de transição entre os tipos espectrais. É importante destacar novamente que não existe, para as matrizes de Jacobi $J_{\delta, \phi}^{j}$, espectro absolutamente contínuo. 


\section{Capítulo 4}

\section{Dimensão Hausdorff da medida de mistura}

Apresentamos neste capítulo uma discussão que envolve a determinação exata da dimensão Hausdorff da medida espectral $\Omega_{00}$ associada à matriz bloco-Jacobi $\mathcal{J}_{\delta, \phi}$. Para tanto, como mencionado no início do Capítulo 3, necessitamos do conhecimento detalhado das medidas espectrais $\mu_{j}$ associadas a cada matriz de Jacobi $J_{\delta, \phi}^{j}$; esta é a linha de investigação da próxima seção. A caracterização da medida espectral $\Omega_{00}$ é somente apresentada na Seção 4.2 .

\subsection{Medidas espectrais $\mu_{j}$}

O comportamento assintótico das soluções linearmente independentes da equação de Schrödinger (3.4), aliado aos resultados de [JL] (apresentados e discutidos no Apêndice H), nos permitem estabelecer precisamente a dimensão Hausdorff de cada medida espectral $\mu_{j}$. Este é precisamente o conteúdo da seguinte proposição.

Proposição 4.1.1 (Proposição 3.9 de [CMW]) Sejam $\mathcal{A}=\left(a_{n}^{\omega}\right)_{n \geq 1}$ o conjunto definido por (1.11) e $\lambda \in \mathbb{R}$. Suponhamos que a seqüência $\left(\theta_{n}^{j}\right)_{n \geq 1}$ dos ângulos de Prüfer definidos por (3.1.20) é u. d. $\bmod \pi$ para todo $\theta_{0}^{j} \in[0, \pi)$ e para quase todo (com respeito $\grave{a}$ medida de Lebesgue) $\varphi_{j} \in[0, \pi)$, onde $2 \cos \varphi_{j}=\lambda_{j} \equiv \lambda-2 \cos (2 \pi j / L)$. Então,

1. existe um autovetor generalizado $u_{j}(\lambda)$ (ou seja, $u_{j}(\lambda)$ é a solução da equação de Schrödinger (3.4) que satisfaz a condição de contorno (1.5)) para a "energia" $\lambda$ tal que as desigualdades

$$
C_{n, j}^{-1} r_{j}^{n / 2} \leq R_{n}^{j}\left(\theta_{\phi}^{j}\right) \leq C_{n, j} r_{j}^{n / 2}
$$

são satisfeitas para a constante $r_{j}>1$, expressa por $(3.1 .30)$, e para $C_{n, j}^{1 / n} \searrow\left(R_{0}^{j}\right)^{2}$ à medida que $n \rightarrow \infty$; 
2. existe uma solução subordinada $v_{j}(\lambda)$ para a "energia" $\lambda$ tal que, para $n$ suficientemente grande, o raio de Prüfer associado a $v_{j}(\lambda)$ satisfaz

$$
\left|R_{n}^{j}\left(\theta_{\alpha}^{j}\right)\right| \leq \tilde{C}_{n, j} r_{j}^{-n / 2}
$$

$\operatorname{com} \alpha \neq \phi$ e $\tilde{C}_{n, j}^{1 / n} \searrow\left(R_{0}^{j}\right)^{2}$ no limite $n \rightarrow \infty$.

Observação 4.1.2 1. A Proposição 4.1.1 é uma otimização do Lema 2.1 de [Z], permitida graças à determinação exata do comportamento assintótico médio (com respeito à condição inicial) da seqüência $\left(R_{n}^{j}\right)_{n \geq 1}$ dos raios de Prüfer (vide o Lema 3.1.11 para maiores detalhes).

2. $\theta_{\phi}^{j}$ representa o ângulo de Prüfer associado ao par de condições iniciais $\mathbf{v}_{0}=$ $(\cos \phi, \sin \phi)$ através da relação (3.1.13).

3. As desigualdades (4.1.1) são satisfeitas para todo $\lambda_{j}=\lambda-2 \cos (2 \pi j / L)$ $\in[-2,2] \backslash 2 \cos (\mathbb{Q} \pi \cap[0, \pi)$ ) (vide o Lema 3.1.11 e o Teorema 3.2.5).

Demonstração. A primeira parte da proposição é simplesmente o Lema 3.1.11. A demonstração da segunda parte segue os mesmos passos do Teorema 3.3.1, que adapta os resultados presentes no Teorema F.2.12 (este fornece uma condição suficiente à existência de uma solução subordinada da equação de Schrödinger (3.4)).

As desigualdades (4.1.1), juntamente com os resultados do Teorema F.3.3, implicam que

$$
C_{n, j}^{-1} r_{j}^{n / 2} \leq t_{j, n} \leq C_{n, j} r_{j}^{n / 2}
$$

com $t_{j, n} \equiv\left\|T\left(a_{n}+1 ; \lambda_{j}\right)\right\|$ e $r_{j}$ dado por (3.1.30). A partir daí, a demonstração da existência da solução subordinada, bem como o conhecimento de seu comportamento assintótico, seguem da demonstração do Teorema 3.3.1.

Podemos finalmente combinar os resultados do Apêndice H à Proposição 4.1.1 e assim determinar exatamente a dimensão Hausdorff de cada medida espectral $\mu_{j}$ :

Teorema 4.1.3 Sejam $J_{P, \phi}^{j}$ as matrizes de Jacobi associadas aos operadores de Schrödinger $\Delta_{P, \phi}^{j}, j \in\{0, \ldots L-1\}$, definidos por (3.2) e que satisfazem a condição de contorno $\phi$ (equação (1.5)), com $P \in \mathcal{P}_{\delta, \beta}$ (ou seja, a seqüência $\left(p_{n}\right)_{n \geq-1}$ satisfaz as relações (1.9)(1.11) com $\delta \in(0,1)$ fixo). Sejam $\mu_{j}$ suas respectivas medidas espectrais. Para qualquer intervalo fechado de energias

$$
L_{j} \subset I_{j} \equiv\left[-2+2 \cos \left(\frac{2 \pi j}{L}\right), 2+2 \cos \left(\frac{2 \pi j}{L}\right)\right],
$$

para quase toda condição de contorno $\phi$ e quase todo $\omega \in \Gamma$, a medida espectral $\mu_{j}$ restrita a $L_{j}$ possui, para todo parâmetro de esparsidade $\beta \geq 2 \in \mathbb{N}$, dimensão Hausdorff

$$
\alpha_{\mu_{j}}\left(\lambda_{j}\right)=1-\frac{\ln r_{j}}{\ln \beta}
$$

com $r_{j}=r\left(\delta, \lambda_{j}\right)<\beta$ dado por (3.1.30). 
Demonstração. Sabemos do Teorema 3.2.5 que a seqüência $\left(\theta_{n}^{j, \omega}\right)_{n \geq 1}$ dos ângulos de Prüfer associada à matriz de Jacobi $J_{\delta, \phi}^{j}$ é u. d. $\bmod \pi$ para todo $\theta_{0}^{j} \in[0, \pi)$, todo $\lambda \in$ $L_{j} \backslash 2 \cos (\mathbb{Q} \pi \cap[0, \pi)$ ) (vide a demonstração do Teorema 3.3.3), e quase todo $\omega \in \Gamma$. Obtemos de (4.1.3) as estimativas

$$
\left\|T\left(k ; \lambda_{j}\right)\right\| \leq C_{n, j} r_{j}^{n / 2} \leq C_{n, j}^{\prime}\left(a_{n}^{\omega}\right)^{\gamma_{j} / 2} \leq C_{n, j}^{\prime} k^{\gamma_{j} / 2}
$$

satisfeitas para todo $\lambda \in L_{j} \backslash 2 \cos (\mathbb{Q} \pi \cap[0, \pi))$ e todo $k \in \mathbb{Z}_{+}$, desde que $a_{n}^{\omega} \leq k<a_{n+1}^{\omega}$, com $\gamma_{j} \equiv \ln r_{j} / \ln \beta, C_{n, j}^{\prime}>0$ para todo $n$ tal que $\lim _{n \rightarrow \infty}\left(C_{n, j}^{\prime}\right)^{1 / n}<\infty$.

Temos em conseqüência da relação acima e da constância da norma $\left\|T\left(k ; \lambda_{j}\right)\right\|$ em $\left[a_{n}^{\omega}+\right.$ $\left.1, a_{n+1}^{\omega}\right]$ (já que a matriz livre $T_{0}$ é equivalente a uma rotação; vide (3.1.4)) a estimativa

$$
\sum_{k=0}^{l_{j}}\left\|T\left(k ; \lambda_{j}\right)\right\|^{2} \leq c l_{j}^{1+\gamma_{j}}
$$

válida para alguma constante $c>0$ e para todo $\lambda \in L_{j} \backslash 2 \cos (\mathbb{Q} \pi \cap[0, \pi))$.

A aplicação da Proposição 4.1 .1 a esses valores de $\lambda=\lambda_{j}+2 \cos (2 \pi j / L)$ assegura a existência de uma solução subordinada $v_{j}^{\text {sub }}$, cujo raio de Prüfer associado satisfaz a estimativa

$$
\left|R_{n}^{j}\left(\theta_{\alpha}^{j}\right)\right|^{2} \leq C_{n}^{\prime \prime}\left(a_{n}^{\omega}\right)^{-\gamma_{j}}
$$

obtemos, usando mais uma vez o fato de toda solução de (3.4) possuir módulo constante no intervalo $\left[a_{n}^{\omega}+1, a_{n+1}^{\omega}\right]$, a desigualdade

$$
\left\|v_{j}^{\mathrm{sub}}\right\|_{l_{j}}^{2} \leq c^{\prime} l_{j}^{1-\gamma_{j}}
$$

válida para alguma constante $c^{\prime}>0$.

Sendo a restrição da medida $\mu_{j}$ ao conjunto $L_{j}$ suportada no conjunto dos $\lambda$ para os quais $v_{j}^{\text {sub }}$ satisfaz a condição de contorno $\phi$ (resultado decorrente do Teorema 3.3.3, que afirma que $\mu_{j}$ não apresenta parte absolutamente contínua, e do Teorema E.11 (o suporte essencial da parte singular do espectro envolve o conjunto dos $\lambda$ aos quais existe

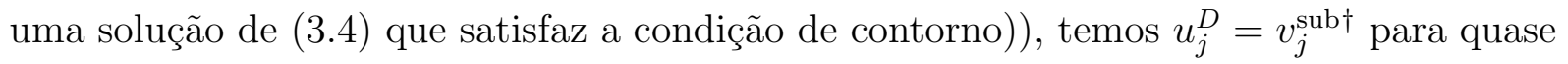
todo $\lambda \in L_{j}$ (devemos excluir um conjunto $A_{j}^{*}$ de medida de Lebesgue nula associado ao suporte essencial do espectro absolutamente contínuo; vide a demonstração do Teorema 3.3.3) e para quase toda condição de contorno $\phi$ (vide a demonstração do Teorema 1.3 de $[\mathrm{JL}])$.

Assim, concluímos de (4.1.6) e (4.1.7) que

$$
\limsup _{l_{j} \rightarrow \infty} \frac{1}{l_{j}^{2-\alpha}} \sum_{k=0}^{l_{j}}\left\|T\left(k ; \lambda_{j}\right)\right\|^{2}<\infty
$$

\footnotetext{
${ }^{\dagger} u_{j}^{D}(\lambda)$ corresponde à solução de (3.4) que satisfaz as condições iniciais (D.1.5).
} 
e que

$$
\liminf _{l_{j} \rightarrow \infty} \frac{\left\|u_{j}^{D}\right\|_{l_{j}}^{2}}{l_{j}^{\alpha}}=0,
$$

desde que $2-\alpha=1+\gamma_{j}+\varepsilon$ e $\alpha=1-\gamma_{j}+\varepsilon$, respectivamente, com $\varepsilon$ um número positivo arbitrário. Dessa forma, temos dos Corolários H.15 (esse fornece uma condição suficiente para que a restrição de uma medida de Borel a um subconjunto da reta seja $\alpha$-contínua) e H. $17^{\ddagger}$ (esse fornece uma condição suficiente para que a restrição de uma medida de Borel a um subconjunto da reta seja $\alpha$-singular) que a medida espectral $\mu_{j}$ é simultaneamente $\left(1-\gamma_{j}-\varepsilon\right)$-contínua e $\left(1-\gamma_{j}+\varepsilon\right)$-singular. Como $\varepsilon$ é arbitrário, segue da Observação G.8 que a restrição $\mu_{j}\left(L_{j} \backslash A_{j}^{1} \cap \cdot\right)$ possui dimensão Hausdorff exata $1-\gamma_{j}\left(A_{j}^{1}\right.$ definido na demonstração do Teorema 3.3.3).

Necessitamos de mais um resultado.

\section{Teorema 4.1.4 (Teorema I.8 de [Si])}

$$
\int_{-\infty}^{\infty}\left[d \mu_{\alpha}(\lambda)\right] d \alpha=d \lambda
$$

no sentido que se $f \in L^{1}(\mathbb{R}, d \lambda)$, então $f \in L^{1}\left(\mathbb{R}, d \mu_{\alpha}\right)$ para quase todo $\alpha, \int f(\lambda) d \mu_{\alpha}(\lambda) \in$ $L^{1}(\mathbb{R}, d \alpha), e$

$$
\int\left(\int f(\lambda) d \mu_{\alpha}(\lambda)\right) d \alpha=\int f(\lambda) d \lambda .
$$

Demonstração. É suficiente demonstrarmos esse resultado, por um argumento elementar, para $f_{z}(\lambda)=(\lambda-z)^{-1}-(\lambda+i)^{-1}, z \in \mathbb{C} \backslash \mathbb{R}$ (devemos usar a analiticidade em $z \mathrm{e}$ o Teorema de aproximação de Stone-Weierstrass). Fechando o contorno de integração no semi-plano superior, obtemos

$$
\int_{-\infty}^{\infty} f_{z}(\lambda) d \lambda=\left\{\begin{array}{cll}
0 & \text { se } & \Im z<0 \\
2 \pi i & \text { se } & \Im z>0
\end{array}\right.
$$

Por outro lado, segue da fórmula de Aronszajn-Krein (vide Seção I.2 de [Si] para a demonstração),

$$
F_{\alpha}(z)=\frac{F(z)}{1+\alpha F(z)}
$$

a identidade

$$
\begin{aligned}
\int f(\lambda) d \mu_{\alpha}(\lambda) & =F_{\alpha}(z)-F_{\alpha}(-i)=\frac{1}{\alpha+F(z)^{-1}}-\frac{1}{\alpha+F(i)^{-1}} \\
& \equiv h_{z}(\alpha),
\end{aligned}
$$

${ }^{\dagger}$ Nele usamos a desigualdade $R_{l_{j}}^{-1}<2 \sqrt{2} / l_{j}$, já que $\left|a_{n}\right|^{-1}>1$ para todo $n \geq 0$.

${ }_{\ddagger}^{\ddagger}$ Nele usamos $R_{l_{j}}^{-1}>2 \sqrt{2}(1-\delta) / l_{j}$, já que $\left|a_{n}\right|^{-1}<(1-\delta)^{-1}$ para todo $n \geq 0$. 
onde $F_{\alpha}(z)=\int d \mu_{\alpha}(x) /(x-z)$.

Agora, se $\pm \Im z>0$, então $\pm \Im F(z)>0$, de modo que $\pm \Im F(z)^{-1}<0$. Dessa forma, $h_{z}(\alpha)$ ou possui dois pólos no semi-plano inferior se $\Im z<0$, ou um em cada semi-plano se $\Im z>0$. O mesmo contorno de integração implica

$$
\int_{-\infty}^{\infty} h_{z}(\alpha) d \alpha=\left\{\begin{array}{ccc}
0 & \text { se } & \Im z<0 \\
2 \pi i & \text { se } & \Im z>0
\end{array}\right.
$$

e assim demonstramos (4.1.11) para $f_{z}(\lambda)$.

Finalmente, segue do Teorema 4.1.4 que $\mu_{j}\left(A_{j}^{1}\right)=0$ para quase todo $\phi$ com respeito à medida de Lebesgue (faça $f(\lambda)=\chi_{B}(\lambda), B \equiv A_{j}^{1}$, em (4.1.11)), e assim para quase todo $\phi$ a restrição $\mu_{j}\left(L_{j} \cap \cdot\right)$ possui (4.1.5) como sua dimensão Hausdorff. Isto conclui a demonstração do Teorema 4.1.3.

\subsection{Medida espectral $\Omega_{00}$}

Os resultados apresentados até o momento (Teoremas 3.3.3 e 4.1.3), apesar de caracterizarem completamente o espectro das matrizes de Jacobi $J_{\delta, \phi}^{j}$, não são suficientes ao estudo dessas propriedades referentes à matriz bloco-Jacobi $\mathcal{J}_{\delta, \phi}$. Para tanto, necessitamos de uma generalização dos resultados de Jitomirskaya e Last, exibidos no Apêndice $\mathrm{H}$, buscando uma análise coletiva das medidas $\mu_{j}$. Começamos pelo

Teorema 4.2.1 (Teorema 3.5 de [CMW]) Seja $\mathcal{J}_{\delta, \phi}$ a matriz bloco-Jacobi definida por (2.1.1), associada ao operador $\Pi_{P, \phi}$ definido por (1.6), com $P \in \mathcal{P}_{\delta, \beta}$, e que satisfaz a condição de contorno (1.5). Sejam $\lambda \in \mathbb{R}$ e $\alpha \in(0,1)$.

Então, para $b=\alpha /(2-\alpha)$,

$$
D_{\Omega_{00}}^{\alpha}(\lambda)=\limsup _{\varepsilon \rightarrow 0} \frac{\Omega_{00}((\lambda-\varepsilon, \lambda+\varepsilon))}{(2 \varepsilon)^{\alpha}}=\infty
$$

se, e somente se, (H.7) segue para ao menos um $j \in \mathcal{I}(\lambda)$, onde

$$
\mathcal{I}(\lambda):=\left\{m \in\{0, \ldots, L-1\}: I_{m} \ni \lambda\right\}
$$

$I_{m}$ definido por (4.1.4).

Demonstração. Suponha que a relação (4.2.1) seja satisfeita. Então, de (3.1), existe ao menos um $j \in \mathcal{I}(\lambda)$ tal que a derivada superior Hausdorff $D_{\mu_{j}}^{\alpha}(\lambda)$ diverge, o que pelo Teorema H.6 (este relaciona o comportamento assintótico das normas das soluções $u^{N}(\lambda)$ e $u^{D}(\lambda)$ à derivada superior Hausdorff (G.3)) ocorre se, e somente se (H.7) é satisfeito. Isso demonstra a necessidade de (H.7). 
Suponha agora que (H.7) seja válido para algum $j \in \mathcal{I}(\lambda)$. Novamente o Teorema H.6 nos leva a identidade (H.6). No entanto, sabemos da relação (3.1) (que expressa $\Omega_{00}$ como um medida de mistura) que se uma das derivadas superiores Hausdorff $D_{\mu_{j}}^{\alpha}(\lambda)$ diverge, necessariamente $D_{\Omega_{00}}^{\alpha}(\lambda)$ diverge, o que implica (4.2.1). Isso demonstra a suficiência de (H.7) e encerra a demonstração do teorema.

Observação 4.2.2 O Teorema 4.2.1 nos diz que quando estudamos o comportamento coletivo da medida $\Omega_{00}$, uma condição necessária e suficiente para que esta seja $\alpha$-singular é que pelo menos uma medida $\mu_{j}$ o seja. Já a $\alpha$-continuidade de $\Omega_{00}$ ocorre numa situação muito mais restrita: todas as medidas $\mu_{j}$ devem ser $\alpha$-contínuas para que $\Omega_{00}$ o seja. Isto está em pleno acordo com a definição de dimensão Hausdorff de uma medida de Borel (vide a Observação G.8), uma vez que podemos entender a continuidade como o comportamento antagônico à singularidade.

Obtemos, a partir do Teorema 4.2.1 e da Observação 4.2.2, a generalização dos Corolários H.13, H.15 e H.16 para a medida $\Omega_{00}$ :

Corolário 4.2.3 (Corolário 3.6 [CMW]) Suponha que para algum $\alpha \in[0,1]$ e todo $\lambda$ pertencente a algum conjunto de Borel $A$, toda solução $v^{j}(\lambda)$ da equação de Schrödinger (3.4) satisfaça

$$
\limsup _{l_{j} \rightarrow \infty} \frac{\left\|v^{j}\right\|_{l}}{l^{2-\alpha}}<\infty
$$

para todo $j \in \mathcal{I}(\lambda)$, com $\|\cdot\|$ alguma norma matricial. Então, a restrição $\Omega_{00}(A \cap \cdot)$ é $\alpha$-contínua.

Demonstração. Como a $\alpha$-continuidade é a "negação" da $\alpha$-singularidade (vide a Observação 4.2.2), segue do Teorema 4.2.1 que a restrição $\Omega_{00}(A \cap \cdot)$ é $\alpha$-contínua se, e somente se, as restrições $\mu_{j}(A \cap \cdot)$ o forem para todo $j \in \mathcal{I}(\lambda)$. No entanto, sabemos, do Corolário H.13, que a condição (4.2.3) implica a $\alpha$-continuidade de $\mu_{j}(A \cap \cdot$ ) (onde usamos a desigualdade $R_{l}^{-1}<2 \sqrt{2} / l$, já que $\left|a_{n}\right|^{-1}>1$ para todo $\left.n \geq 0\right)$. Isso encerra a demonstração.

Corolário 4.2.4 (Corolário 3.7 de [CMW]) Suponha que para algum $\alpha \in[0,1]$ e todo $\lambda$ pertencente a algum conjunto de Borel $A$, a desigualdade

$$
\limsup _{l_{j} \rightarrow \infty} \frac{1}{l^{2-\alpha}} \sum_{n=0}^{l}\left\|T_{j}(n ; \lambda)\right\|^{2}<\infty
$$

seja satisfeita para todo $j \in \mathcal{I}(\lambda)$, com $\|\cdot\|$ alguma norma matricial. Então, a restrição $\Omega_{00}(A \cap \cdot)$ é $\alpha$-contínua. 
Demonstração. O Corolário 4.2.4 é conseqüência direta dos Corolários 4.2.3 e H.15.

Corolário 4.2.5 (Corolário 3.8 de [CMW]) Suponha que, para ao menos um $j \in$ $\mathcal{I}(\lambda) \neq \emptyset$, tenhamos

$$
\liminf _{l_{j} \rightarrow \infty} \frac{\left\|u_{j}^{D}\right\|_{l}^{2}}{l^{\alpha}}=0
$$

para todo $\lambda$ pertencente a algum conjunto de Borel $A$. Então, a restrição $\Omega_{00}(A \cap \cdot)$ é $\alpha$-singular.

Demonstração. O Corolário 4.2.5 é conseqüência direta do Corolário H.16 (onde novamente usamos $R_{l}^{-1}>2 \sqrt{2}(1-\delta) / l$, uma vez que $\left|a_{n}\right|^{-1}<(1-\delta)^{-1}$ para todo $\left.n \geq 0\right)$ e do Teorema 4.2.1.

Somos finalmente capazes de enunciar o

Teorema 4.2.6 Seja $\mathcal{J}_{\delta, \phi}$ a matriz bloco-Jacobi definida por (2.1.1), associada ao operador de Schrödinger $\Pi_{\delta, \phi}$ definido por (1.6), com $P \in \mathcal{P}_{\delta, \beta}$ (isto é, satisfazendo as relações (1.9)-(1.11), com $\delta \in(0,1)$ ) e que satisfaz as condições de contorno (1.16) e (1.17). Seja $\Omega$ sua respectiva medida espectral matricial e seja $B^{(k)}$ o conjunto tal que a multiplicidade espectral de $\chi_{B^{(k)}} \tilde{\Pi}_{P, \phi}$ é $k$ (vide os Lemas 2.3.6 e 2.3.8 para a notação). Para qualquer intervalo fechado de energias

$$
I \subset \bigcup_{j}\left[-2+2 \cos \left(\frac{2 \pi j}{L}\right), 2+2 \cos \left(\frac{2 \pi j}{L}\right)\right],
$$

para quase toda condição de contorno $\phi$ e quase todo $\omega \in \Gamma$, o elemento $\Omega_{00}$ da medida espectral $\Omega$ restrito a $I^{(k)}=I \cap B^{(k)}$ possui, para todo parâmetro de esparsidade $\beta \geq 2 \in \mathbb{N}$, dimensão Hausdorff exata

$$
\alpha_{\Omega_{00}}(\lambda)=\min _{j \in \mathcal{I}(\lambda)} \alpha_{\rho_{j}}\left(\lambda_{j}\right)=\min _{j \in \mathcal{I}(\lambda)}\left(1-\frac{\ln r_{j}}{\ln \beta}\right),
$$

com $\mathcal{I}(\lambda)$ definido por (4.2.2) e $r_{j}=r\left(\delta, \lambda_{j}\right)$ dado por (3.1.30).

Observação 4.2.7 1. O Teorema 4.2.6 corresponde a uma adaptação do Teorema 3.11 de [CMW] ao operador cujas barreiras são aleatórias. No caso tratado por Carvalho, Marchetti e Wreszinski, a dimensão Hausdorff pode ser obtida com precisão arbitrária, desde que tomemos o parâmetro de esparsidade $\beta$ suficientemente grande.

2. O Teorema 4.2.6 generaliza (do caso unidimensional ao problema na faixa finita) o Teorema 6.3 de [Z]. 
3. A medida $\Omega_{00}$ restrita a $I^{(k)}$ possui, para multiplicidade espectral $k>1$, dimensão Hausdorff local uniforme para todas as componentes.

4. Optamos por definir a dimensão Hausdorff da medida $\Omega_{00}$ como o valor abaixo do qual todas as medidas $\mu_{j}$ são contínuas e acima do qual ao menos uma destas é singular. Isto está de acordo com os resultados do Teorema 4.2.1 e de seus subseqüentes corolários, que por conseguinte concordam com o conceito de continuidade e de singularidade com respeito a uma medida Hausdorff presentes no Apêndice G vide o Corolário G.10). Caso redefiníssemos tais conceitos para o caso de uma mistura de medidas (em particular para aquela que define a medida $\Omega_{00}$ ), certamente teríamos outros resultados.

Demonstração. Assim como no Teorema 4.1.3, é possível mostrar que

$$
\sum_{k=0}^{l_{j}}\left\|T\left(k ; \lambda_{j}\right)\right\|^{2} \leq c l_{j}^{1+\gamma_{j}}
$$

estimativa válida para todo $j=0,1, \ldots, L-1$, para alguma constante $c>0$ e para todo $\lambda \in I_{j} \backslash 2 \cos (\mathbb{Q} \pi \cap[0, \pi))$.

Assumamos que $I^{(k)}=I \cap B^{(k)} \neq \emptyset$ para algum $k \in\{1, \ldots, L\}$. A aplicação da Proposição 4.1.1, para $\lambda \in I^{(k)}$, garante a existência de $k$ soluções subordinadas $u_{j_{1}}^{\text {sub }}, \ldots, u_{j_{k}}^{\text {sub }}$, não necessariamente distintas ${ }^{\dagger}$, tais que $j_{\ell} \in \mathcal{I}(\lambda)$ e

$$
R_{n}^{j_{\ell}}\left(\theta_{\alpha^{*}}^{j_{\ell}}\right) \leq C_{n, j}^{\prime \prime \prime} a_{n}^{-\gamma_{j_{\ell}} / 2}
$$

(para alguma $\alpha^{*}$-condição de contorno) seguem para todo $\ell \in\{1, \ldots k\}$. Como toda solução da equação de Schrödinger (3.4) apresenta módulo constante no intervalo $\left[a_{n}+\right.$ $\left.1, a_{n+1}\right]$, temos

$$
\left\|u_{j_{\ell}}^{\mathrm{sub}}\right\|_{l_{j_{\ell}}}^{2} \leq c^{\prime} l_{j_{\ell}}^{1-\gamma_{\ell_{j}}}
$$

para algum $c^{\prime}>0$.

As mesmas considerações presentes na demonstração do Teorema 4.1.3 nos permitem concluir que as relações

$$
\begin{gathered}
\limsup _{l_{j_{\ell}} \rightarrow \infty} \frac{1}{l_{j_{\ell}}^{2-\alpha}} \sum_{k=0}^{l_{j_{\ell}}}\left\|T_{j_{\ell}}(k ; \lambda)\right\|^{2}<\infty, \\
\liminf _{l_{j_{\ell}} \rightarrow \infty} \frac{\left\|u_{j_{\ell}}^{D}\right\|_{l_{j_{\ell}}}^{2}}{l_{j_{\ell}}^{\alpha}}=0
\end{gathered}
$$

\footnotetext{
${ }^{\dagger}$ Se $i, j \in\{0, \ldots, L-1\}, i<j$, são tais que $i=L-j$, então $\lambda_{i}=\lambda_{j}$, de modo que, pela Proposição 4.1.1, $u_{i}^{\text {sub }}=u_{j}^{\text {sub }}$.
} 
são satisfeitas para quase todo $\omega \in \Gamma$, para cada $\ell \in\{1, \ldots, k\}$ e para todo $\lambda \in$ $\bigcup_{\ell=1}^{k}\left(I_{j_{\ell}} \backslash A_{j_{\ell}}^{1}\right)\left(A_{j}^{1}\right.$ o conjunto de medida de Lebesgue nula presente na demonstração do Teorema 4.1.3), desde que $2-\alpha>1+\gamma_{l_{j_{\ell}}}$ e $\alpha>1-\gamma_{j_{\ell}}$.

O Corolário 4.2.4 nos diz que se (4.1.8) é satisfeita para todo $j_{\ell} \in \mathcal{I}(\lambda)$, a restrição $\Omega_{00}\left(\left(I \backslash \bigcup_{\ell} A_{\theta_{j_{\ell}}^{1}}\right) \cap \cdot\right)$ é $\alpha$-contínua. Claramente $\alpha=\min _{\ell \in\{1, \ldots, k\}}\left(1-\gamma_{j_{\ell}}\right)$ é suficiente:

$$
\limsup _{l_{j_{\ell}} \rightarrow \infty} \frac{1}{l_{j_{\ell}}^{2-\alpha}} \sum_{n=1}^{l_{j_{\ell}}}\left\|T_{j_{\ell}}(n ; \lambda)\right\|^{2} \leq \limsup _{l_{j_{\ell}} \rightarrow \infty} \frac{1}{l_{j_{\ell}}^{1+\gamma_{j_{\ell}}}} \sum_{n=1}^{l_{j_{\ell}}}\left\|T_{j_{\ell}}(n ; \lambda)\right\|^{2}<\infty,
$$

e assim, a desigualdade (4.1.8) é satisfeita simultaneamente para todo $j_{\ell} \in \mathcal{I}(\lambda)$ desde que $\lambda \in I^{(k)} \backslash \bigcup_{\ell} A_{\theta_{j_{\ell}}^{1}}$. Desse modo, a restrição $\Omega_{00}\left(\left(I^{(k)} \backslash \bigcup_{\ell} A_{\theta_{j_{\ell}}^{1}}\right) \cap \cdot\right)$ é no máximo $\alpha$-contínua (vide a Observação G.8).

Afirmamos que $\Omega_{00}\left(\left(I^{(k)} \backslash \bigcup_{\ell} A_{\theta_{j_{\ell}}^{1}}\right) \cap \cdot\right)$ é ao menos $\alpha$-singular, com $\alpha=\min _{\ell \in\{1, \ldots k\}}(1-$ $\gamma_{j_{\ell}}$ ). Temos do Corolário 4.2.5 que a restrição acima é $\eta$-singular para todo $\eta>\alpha$, uma vez que a relação (4.2.4) é satisfeita para todo $j_{\ell}, \operatorname{com} \ell \in\{1, \ldots, k\}$ (na verdade, bastaria ser satisfeita para apenas um $j_{\ell}$ ), o que demonstra nossa afirmação.

Portanto, da Definição G.7, concluímos que $\Omega_{00}\left(\left(I^{(k)} \backslash \bigcup_{\ell} A_{\theta_{j_{\ell}}^{1}}\right) \cap \cdot\right)$ possui dimensão exata

$$
\alpha=\min _{\ell \in\{1, \ldots, k\}}\left(1-\gamma_{j_{\ell}}\right)
$$

o que, juntamente com a definição de $\gamma_{j}$, é justamente (4.2.6), uma vez que, por hipótese, $y_{j_{\ell}}(\lambda)>0$ para $\ell \in\{1, \ldots, k\}$.

Como no Teorema 4.1.3, segue do Teorema 4.1.4 que $\Omega_{00}\left(\bigcup_{\ell} A_{\theta_{j_{\ell}}^{1}}\right)=0$ é satisfeito para quase todo $\phi$, e assim para quase todo $\phi$ a restrição $\Omega_{00}\left(I^{(k)} \cap \cdot\right)$ possui (4.2.6) como sua dimensão Hausdorff. Isto conclui a demonstração do teorema.

Uma conclusão bastante interessante extraída do Teorema 4.2.6 é o fato da medida espectral $\Omega_{00}$ sempre herdar o comportamento mais singular entre os seus componentes. Expliquemos o que isso significa.

Seja $B$ um conjunto de Borel, $B \subset I$ ( $I$ dado por (4.1.4)). Se $\alpha_{\Omega_{00}}(\lambda)>0$ para todo $\lambda \in B$, então $\Omega_{00}(B \cap \cdot)$ é puramente singular-contínuo. Vemos de (4.2.6) e de (3.1.30) que a condição anterior é satisfeita se, e somente se, a desigualdade

$$
\left(4-\lambda_{j}^{2}\right)(\beta-1)>\left(\frac{1-p^{2}}{p}\right)^{2}
$$

é válida para todo $j \in \mathcal{K}(\lambda):=\left\{m \in \mathcal{I}(\lambda): y_{m}(\lambda)>0\right\}$. Esta é exatamente a desigualdade (3.3.19), que como vimos no Teorema 3.3.3, representa uma condição necessária à existência do espectro singular-contínuo. Logo, se a desigualdade (4.2.8) deixa de ser satisfeita para alguma vizinhança de $\lambda_{j}$, então o espectro de $\Omega_{00}(B \cap \cdot)$ se torna puramente pontual nesse intervalo. Este resultado é conseqüência direta do Corolário 4.2.5. Ilustremos esse fato com um exemplo. 


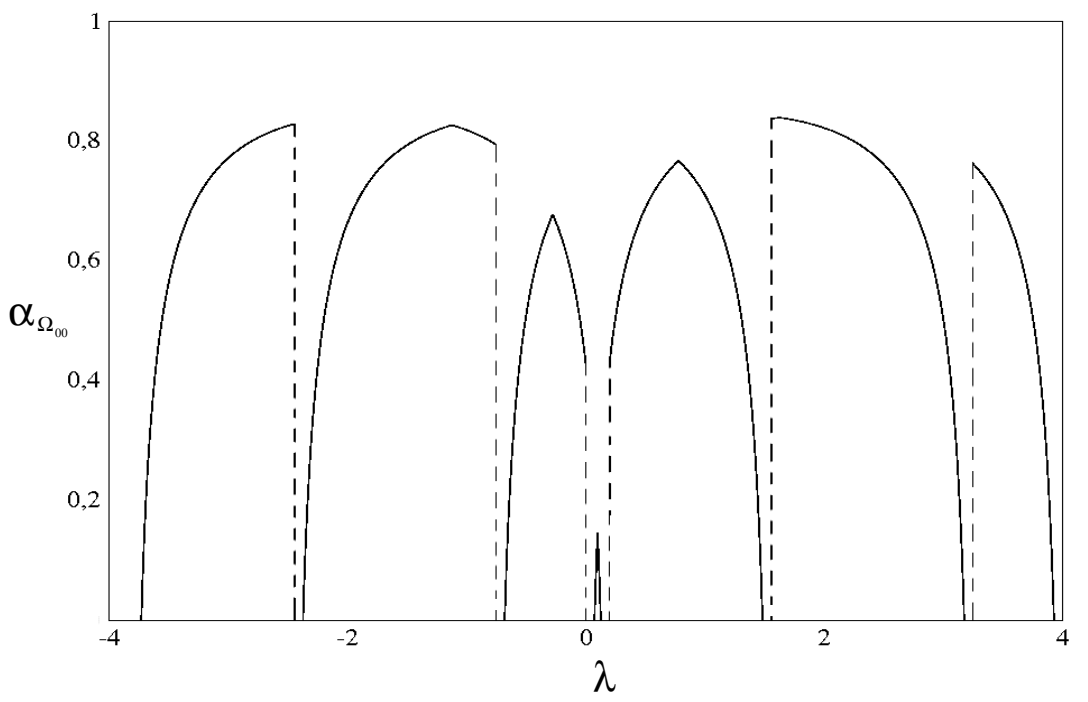

Figura 4.1: Gráfico da dimensão Hausdorff local $\alpha_{\Omega_{00}}$ como função do parâmetro espectral $\lambda$ para uma faixa de tamanho $L=7$, parâmetro de esparsidade $\beta=5$ e $\delta=0,4$.

Repare que, na figura acima, as regiões em que $\alpha_{\Omega_{00}}$ se anula são justamente aquelas onde existe ao menos um componente unidimensional de $\mathcal{J}_{\delta, \phi}$ com espectro puramente pontual. Essa condição garante uma dimensão nula nas proximidades do centro do espectro, algo que não esperaríamos caso estivéssemos analisando cada matriz de Jacobi $J_{\delta, \phi}^{j}$ independentemente (onde o espectro é menos singular no centro e mais singular nas extremidades). 


\section{Capítulo 5}

\section{Modelos com esparsidades sub-geométrica e super-geométrica}

Esse capítulo é dedicado ao estudo da medida espectral matricial $\Omega$ associada à matriz bloco-Jacobi $\mathcal{J}_{\delta, \phi}$ definida por (2.1.1) quando a esparsidade das barreiras (1.9) é tomada como sub ou super-geométrica, em substituição ao caráter geométrico exibido em (1.10).

O conjunto aleatório $\mathcal{A}=\left\{a_{n}^{\omega}\right\}_{n \geq 1}$ de números naturais $a_{n}^{\omega}=a_{n}+\omega_{n}$ é agora admissível caso a condição

$$
a_{n}-a_{n-1}=\left[e^{c n^{\gamma}}\right]
$$

seja satisfeita para todo $n \geq 1$, com $c, \gamma>0$ constantes e $\omega_{n}$, como antes, variáveis aleatórias independentes e uniformemente distribuídas em $\Gamma_{n}=\{-n, \ldots, n\}$. A seqüência definida por (5.1) cresce sub ou super-geometricamente rápido caso $\gamma<1$ ou $\gamma>1$, e coincide com a definida anteriormente caso $\gamma=1$ e $c=\ln \beta$. Poderíamos lidar com outras condições de esparsidade com diferentes taxas de crescimento através dos mesmos métodos empregados aqui; no entanto, a condição (5.1) é suficientemente abrangente para nossos propósitos.

É bastante simples notar que todos os resultados que envolvem a caracterização da matriz espectral $\Omega$, presentes na Seção 2.3, permanecem válidos. Em particular, estudamos individualmente, como nas Seções 3 e 4, as características das medidas $\mu_{j}$, $j \in\{0, \ldots, L-1\}$, associadas às matrizes de Jacobi $J_{\delta, \phi}^{j}$, e em seguida seu comportamento coletivo através da medida de "mistura" $\Omega_{00}$ (vide a equação (3.1)), de importância ímpar (vide a Proposição 2.3.2) na determinação da natureza espectral.

Seja $J_{\delta, \phi}^{j}$ a matriz de Jacobi associada ao operador $\Delta_{\delta, \phi}^{j}$, definida por (3.3), (1.9) e (1.5), com a condição (1.11) substituída por (5.1). Permitimos também a variação de $\delta$ na equação (1.9) em algumas situações particulares.

Como a demonstração do Teorema 3.2 continua válida em todas estas situações, o espectro essencial de $J_{\delta, \phi}^{j}$ continua como antes: $\sigma_{\text {ess }}\left(J_{\delta, \phi}^{j}\right)=[-2+2 \cos (2 \pi j / L), 2+$ $2 \cos (2 \pi j / L)]$ 


\subsection{Caso $\gamma<1$}

Começamos pelo

Teorema 5.1.1 (Teorema 4.1 de [CMW1]) Seja $J_{\delta, \phi}^{j}$ a matriz de Jacobi definida por (3.3), com a condição de esparsidade (1.11) substituída por (5.1), onde $0<\gamma<1$ e c $>0$. Então, a medida espectral $\mu_{j}$ é puramente pontual quando restrita ao espectro essencial de $J_{\delta, \phi}^{j}$ (vide Teorema 3.2) para quase toda condição de contorno $\phi \in[0, \pi)$ e quase todo $\omega \in \Gamma=\underset{n=1}{\times}\{-n, \ldots, n\}$ com respeito à medida produto $\nu$.

Demonstração. Este teorema é uma extensão do ítem (b) do Teorema 3.3.3 para a situação de esparsidade sub-geométrica. De acordo com o Teorema 3.3.1, é suficiente mostrar que a condição (3.3.3) é satisfeita para todo $\lambda_{j}=\lambda-2 \cos (2 \pi j / L) \in[-2,2]$, a menos de um conjunto de medida de Lebesgue nula, e para quase toda condição de contorno $\phi \in[0, \pi)$ (substituímos a condição de esparsidade (1.11) por (5.1)). O Teorema 3.2 completa a demonstração.

Se $n$ é tal que as desigualdades $a_{N}^{\omega} \leq n<a_{N+1}^{\omega}$ são satisfeitas para algum $N \in \mathbb{N}$, temos do Lema 3.1.11, do Teorema 3.2.5 e das relações (3.1.30), (3.3.3), (3.3.13), (3.3.14), (3.3.22), (3.3.23) e (5.1) a estimativa

$$
\begin{array}{r}
\sum_{N=1}^{\infty}\left(e^{c N^{\gamma}}+\omega_{j}-\omega_{j-1}\right)\left\|T\left(a_{N} ; \lambda_{j}\right)\right\|^{2}\left(\sum_{m=N}^{\infty}\left\|T\left(a_{m} ; \lambda_{j}\right)\right\|^{-2}\right)^{2} \\
\leq C_{j}^{\prime} \sum_{N=1}^{\infty}\left(e^{c N^{\gamma}}+2 N\right) C_{N, j} r_{j}^{N} S^{2} \\
\leq C_{j}^{\prime \prime} \sum_{N=1}^{\infty}\left(e^{c N^{\gamma}}+2 N\right)\left(C_{N, j}\right)^{3} r_{j}^{-N}
\end{array}
$$

(as contantes acima são definidas como em (3.3.24)). Fica claro que (5.1.1) converge para todo $\gamma$ tal que $0<\gamma<1$, e para todo $\lambda_{j}$ pertencente ao conjunto

$$
B_{j}=I_{j} \backslash\left(A_{j}^{1} \cap I_{j}\right)
$$

$A_{j}^{1}$ um conjunto de medida de Lebesgue nula (vide a demonstração do Teorema 3.3.3); conseqüentemente, pelo Teorema 3.3.1, a equação de Schrödinger (3.4) possui uma solução $l^{2}\left(\mathbb{Z}_{+}, \mathbb{C}\right)$. Finalmente, uma vez que, pelo Teorema $3.2, I_{j} \subseteq \sigma_{\text {ess }}\left(J_{\delta, \phi}^{j}\right)$ para toda condição de contorno $\phi \in[0, \pi)$, as hipóteses da Proposição 3.3.5 são satisfeitas e $J_{\delta, \phi}^{j}$ possui apenas espectro puramente pontual em $I_{j}$ para quase todo $\phi \in[0, \pi)$. Concluímos, portanto, a demonstração do teorema.

Observação 5.1.2 O espectro puramente pontual obtido no Teorema 5.1 .1 não se altera mediante qualquer perturbação $\delta<1$ (vide a definição (1.9)). Um espectro menos singular pode ser obtido se fizermos $\delta_{k}$ tender a 1 dependendo, no entanto, de $a_{k}^{\omega}$. 
Sendo cada uma das medidas $\mu_{j}$ puramente pontuais, concluímos das discussões presentes no Capítulo 4, da equação (2.4.3) e dos Teoremas 2.4.5 e 5.1.1 o seguinte

Teorema 5.1.3 Seja $\mathcal{J}_{\delta, \phi}, \delta \in(0,1)$, a matriz bloco-Jacobi definida por (2.1.1), associada ao operador $\Pi_{\delta, \phi}$, que satisfaz as condições de contorno (1.16) e (1.17), com a condição de esparsidade (1.11) substituída por (5.1), onde $0<\gamma<1$ e c $>0$. Então, a medida espectral $\Omega_{00}$ é puramente pontual quando restrita ao espectro essencial de $\mathcal{J}_{\delta, \phi}^{j}$ (vide Teorema 2.4.5) para quase toda condição de contorno $\phi \in[0, \pi)$ e quase todo $\omega \in \Gamma=\underset{n=1}{\infty}\{-n, \ldots, n\}$ com respeito à medida produto $\nu$.

\subsection{Casos em que $\gamma>1$}

Consideremos agora as matrizes de Jacobi $J_{\delta, \phi}^{j}$, para cada $j \in\{0, \ldots L-1\}$, sujeitas à condição de esparsidade super-geométrica (5.1), $\operatorname{com} \gamma>1$. As características da medida espectral de $J_{\delta, \phi}^{j}$ são determinadas, nesta situação, pelo

Teorema 5.2.1 (Teorema 4.2 de [CMW1]) Sejam as matrizes de Jacobi $J_{\delta, \phi}^{j}$ definidas por (3.3), associadas aos operadores $\Delta_{\delta, \phi}^{j}(j \in\{0, \ldots, L-1\})$ definidos como no Teorema 5.1.1, com $\gamma>1$ e $c>0$. Nesse caso, as medidas espectrais $\mu_{j}$ são puramente singular-contínuas, com dimensão Hausdorff igual a 1, para quase todo $\lambda_{j}=$ $\lambda-2 \cos (2 \pi j / L) \in[-2,2]$ e para quase todo $\omega \in \Gamma \underset{n=1}{\infty}\{-n, \ldots, n\}$ com respeito à medida produto $\nu$.

Observação 5.2.2 O Teorema 5.2.1 é, na realidade, uma variação do Teorema 4.2 de [CMW1]. Este último é, de fato, mais geral, já que os resultados apresentados valem para todo $\omega \in \Gamma=\underset{n=1}{\infty}\{-n, \ldots, n\}$.

Demonstração. Comecemos demonstrando que o espectro essencial de $J_{\delta, \phi}^{j}$ é puramente singular-contínuo no intervalo $I_{j}=[-2+2 \cos (2 \pi j / L), 2+2 \cos (2 \pi j / L)], j \in\{0, \ldots, L-$ 1\}. Para tanto, pelo Teorema F.2.17, é suficiente mostrar que $\sum_{k=n}^{\infty}\left\|T_{j}(n ; \lambda)\right\|^{-2}=\infty$ para todo $\lambda \in[-2,2]$, a menos de um conjunto de medida de Lebesgue nula. Segue mais uma vez do Lema 3.1.11, do Teorema 3.2.5, das relações (3.3.12), (3.3.14), da definição (3.3.15) e das desigualdades (3.3.16) (onde substituímos a condição de esparsidade geométrica (1.11) pela condição super-geométrica (5.1)), a estimativa

$$
\tilde{C}_{j}^{-2} \sum_{l=N+1}^{\infty} C_{l, j}^{-1} e^{c l \gamma} r_{j} \leq \sum_{k=n}^{\infty}\left\|T_{j}(k ; \lambda)\right\|^{-2},
$$

válida para $a_{N}^{\omega} \leq n<a_{N+1}^{\omega}$ e para quase todo $\omega \in \Gamma$ com respeito à medida produto $\nu$ (as constantes $\tilde{C}_{j}, C_{l, j}$ e $r_{j}$ são definidas como na demonstração do Teorema 3.3.3).

Como o membro esquerdo da estimativa acima diverge, concluímos que o espectro de $J_{\delta, \phi}^{j}$ é inteiramente singular-contínuo no intervalo $I_{j} \backslash A_{j}^{1}, A_{j}^{1}$ um conjunto de medida de Lebesgue nula (vide a demonstração do Teorema 3.3.3). 
Resta-nos demonstrar que a dimensão Hausdorff da restrição $\mu_{j}\left(I_{j} \cap \cdot\right)$ é 1. Segue das mesmas considerações do Teorema 4.1 .3 e da condição de esparsidade (5.1) a estimativa

$$
\left\|T_{j}(k ; \lambda)\right\| \leq C_{n, j} r_{j}^{n / 2} \leq C_{n, j}^{\prime}\left(a_{n}^{\omega}\right)^{\left(a_{n}^{\omega}\right)^{1 / \gamma-1} \ln r_{j} / 2} \leq C_{n, j}^{\prime} k^{(k-1)^{1 / \gamma-1} \ln r_{j} / 2},
$$

válida para todo $\lambda \in I_{j} \backslash 2 \cos (\mathbb{Q} \pi \cap[0, \pi))$, quase todo $\omega \in \Gamma=\underset{n=1}{\times}\{-n, \ldots, n\}$, e todo $k \in \mathbb{Z}_{+}$tal que $a_{n}^{\omega} \leq k<a_{n+1}^{\omega}$, com $C_{n, j}^{\prime}>0$ tal que $\lim _{n \rightarrow \infty}\left(C_{n, j}^{\prime}\right)^{n=1}<\infty$.

Temos, em conseqüência da relação acima e da constância da norma $\left\|T_{j}(k ; \lambda)\right\|$ em $\left[a_{n}^{\omega}+1, a_{n+1}^{\omega}\right]$

$$
\sum_{k=0}^{l_{j}}\left\|T_{j}(k ; \lambda)\right\|^{2} \leq c l_{j}^{1+l_{j}^{1 / \gamma-1} \ln r_{j}}
$$

estimativa válida para alguma constante $c>0$ e para todo $\lambda \in I_{j} \backslash 2 \cos (\mathbb{Q} \pi \cap[0, \pi))$.

A aplicação da Proposição 4.1 .1 a esses valores de $\lambda_{j}$ assegura a existência de uma solução subordinada $v_{j}^{\text {sub }}$, cujo raio de Prüfer associado satisfaz a estimativa

$$
\left|R_{n}^{j}\left(\theta_{\alpha^{*}}^{j}\right)\right|^{2} \leq C_{n}^{\prime \prime}\left(a_{n}^{\omega}\right)^{-\left(a_{n}^{\omega}\right)^{1 / \gamma-1} \ln r_{j} / 2}
$$

Já que toda solução de (3.4) possui módulo constante no intervalo $\left[a_{n}^{\omega}+1, a_{n+1}^{\omega}\right]$, obtemos

$$
\left\|v_{j}^{\mathrm{sub}}\right\|_{l_{j}}^{2} \leq c^{\prime} l_{j}^{1-l_{j}^{1 / \gamma-1} \ln r_{j}}
$$

para alguma constante $c^{\prime}>0$.

Por fim, obtemos de (5.2.1) e (5.2.2) (seguindo os mesmos passos da demonstração do Teorema 4.1.3) as relações

$$
\limsup _{l_{j} \rightarrow \infty} \frac{1}{l_{j}^{2-\alpha}} \sum_{k=0}^{l_{j}}\left\|T_{j}(k ; \lambda)\right\|^{2} \leq \limsup _{l_{j} \rightarrow \infty} c l_{j}^{\left(l_{j}^{1 / \gamma-1}-1+\alpha\right)}<\infty,
$$

e

$$
\liminf _{l_{j} \rightarrow \infty} \frac{\left\|u_{j}^{D}\right\|_{l_{j}}^{2}}{l_{j}^{\alpha}}=\liminf _{l_{j} \rightarrow \infty} l_{j}^{\left(1-\alpha-l_{j}^{1 / \gamma-1}\right)}=0
$$

válidas para todo $\lambda \in A_{j}^{1}$ (a identificação $u_{j}^{D}=v_{j}^{\text {sub }}$ se dá a menos de um conjunto de medida de Lebesgue nula; vide a demonstração do Teorema 4.1.3), caso $\alpha<1-\varepsilon$ e $\alpha>1+\varepsilon$, respectivamente, com $\varepsilon>0$. Dessa forma, segue dos Corolários H.15 (onde usamos a desigualdade $R_{l_{j}}^{-1}<2 \sqrt{2} / l_{j}$, já que $\left|a_{n}\right|^{-1}>1$ para todo $n \geq 0$ ) e H.17 (onde usamos $R_{l_{j}}^{-1}>2 \sqrt{2}(1-\delta) / l_{j}$, já que $\left|a_{n}\right|^{-1}<(1-\delta)^{-1}$ para todo $\left.n \geq 0\right)$ que a medida espectral $\mu_{j}$ é simultaneamente $(1-\varepsilon)$-contínua e $(1+\varepsilon)$-singular. Como $\varepsilon$ é arbitrário, concluímos da Observação G.8 que a restrição $\mu_{j}\left(I_{j} \backslash A_{1}^{j} \cup \cdot\right)$ possui dimensão Hausdorff exata 1.

Finalmente, segue do Teorema 4.1.4 que $\mu_{j}\left(A_{j}^{1}\right)=0$ para quase todo $\phi$ com respeito à medida de Lebesgue (faça $f(\lambda)=\chi_{B}(\lambda), B \equiv A_{j}^{1}$, em (4.1.11)), e assim para quase 
todo $\phi$, a restrição $\mu_{j}\left(I_{j} \cap \cdot\right)$ possui dimensão Hausdorff 1. Isto conclui a demonstração do teorema.

Obtemos, pelas mesmas considerações que nos levam ao Teorema 5.1.3, o

Teorema 5.2.3 Seja $\mathcal{J}_{\delta, \phi}$ a matriz bloco-Jacobi definida por (2.1.1), associada ao operador de diferença finita $\Pi_{\delta, \phi}$ definido como no Teorema 5.1.3, com $\gamma>1$ e c $>0$. Nesse caso, a medida espectral $\Omega_{00}$ é puramente singular-contínua, com dimensão Hausdorff igual a 1 para quase todo $\lambda$ pertencente ao intervalo definido por (2.4.3), e quase todo $\omega \in \Gamma=\underset{n=1}{\times}\{-n, \ldots, n\}$ com respeito à medida produto $\nu$.

Observação 5.2.4 Podemos, de fato, substituir a condição para quase todo pela condição para todo $\omega \in \Gamma=\underset{n=1}{\infty}\{-n, \ldots, n\}$ (vide a Observação 5.2.2).

Mostramos a seguir que a perturbação esparsa pode ser escolhida de tal modo que a medida espectral, apesar de puramente singular-contínua, apresente dimensão Hausdorff 0 . Para tanto, façamos a seqüência $P=\left(p_{n}\right)_{n \geq-1}$ variar de acordo com $a_{k}^{\omega} \in \mathcal{A}$ :

$$
p_{n}= \begin{cases}q_{k} & \text { se } \\ 1 & n=a_{k}^{\omega} \in \mathcal{A} \\ & \text { de outra forma }\end{cases}
$$

onde $q_{k}$ tende a zero super-geometricamente rápido à medida que $k \rightarrow \infty$; a saber, adotemos $q_{k}$ tal que as desigualdades

$$
e^{c n^{\gamma}-c_{1} n^{\delta}} \leq \prod_{k=0}^{n} q_{k}^{-2} \leq e^{c n^{\gamma}-c_{2} n^{\delta}}
$$

sejam satisfeitas para constantes $c_{1} \geq c_{2}>0$ e $\gamma>\delta>1$, com $\delta>\gamma-1$. Nesse caso, a função $h(\theta)$ definida no Lema 3.1.7 passa a ser

$$
\begin{aligned}
h_{k}\left(\theta_{k}^{j}\right) & =\ln \left(q_{k}^{4} \cos ^{2} \theta_{k}^{j}+\left(\sin \theta_{k}^{j}+\left(1-q_{k}^{2}\right) \cot \varphi_{j} \cos \theta_{k}^{j}\right)^{2}\right) \\
& \equiv \ln \left(a_{k}^{j}+b_{k}^{j} \cos 2 \theta_{k}^{j}+c_{k} \sin 2 \theta_{k}^{j}\right)
\end{aligned}
$$

onde

$$
\begin{aligned}
2 a_{k}^{j} & =\left(1-q_{k}^{2}\right)^{2} \cot ^{2} \varphi_{j}+1+q_{k}^{4} \\
2 b_{k}^{j} & =\left(1-q_{k}^{2}\right)^{2} \cot ^{2} \varphi_{j}-1+q_{k}^{4} \\
c_{k}^{j} & =\left(1-q_{k}^{2}\right) \cot \varphi_{j}
\end{aligned}
$$

satisfazem a identidade

$$
\left(a_{k}^{j}\right)^{2}=\left(b_{k}^{j}\right)^{2}+\left(c_{k}^{j}\right)^{2}+q_{k}^{4} .
$$

Um cálculo explícito nos leva a

$$
a_{k}^{j}-\sqrt{\left(a_{k}^{j}\right)^{2}-q_{k}^{4}} \leq a_{k}^{j}+b_{k}^{j} \cos 2 \theta_{j}+c_{k}^{j} \sin 2 \theta_{j} \leq a_{k}^{j}+\sqrt{\left(a_{k}^{j}\right)^{2}-q_{k}^{4}}=\sin ^{-2} \varphi_{j}\left(1+O\left(q_{k}^{2}\right)\right),
$$

o que nos permite chegar à seguinte conclusão: 
Teorema 5.2.5 (Teorema 5.4 de [CMW1]) Seja $J_{\delta, \phi}^{j}$ a matriz de Jacobi definida por (3.3), associada ao operador de diferença finita $\Delta_{P, \phi}^{j}$ definido por (3.2) e que satisfaz a condição de contorno (1.5), com $P=\left(p_{n}\right)_{n \geq-1}$ dado por (5.2.3) e sujeito à condição de esparsidade (5.1) $(\gamma>1$ e $c>0)$. Então, para quase toda condição de contorno $\phi \in[0, \pi)$ e todo $\omega \in \Gamma={\underset{x=1}{\infty}\{}_{n}^{\infty}\{-n, \ldots, n\}$ com respeito a $\nu$, a medida espectral $\mu_{j}$ restrita ao intervalo $I_{j}=[-2+2 \cos (2 \pi j / L), 2+2 \cos (2 \pi j / L)]$ é puramente singular-contínua $e$ possui dimensão Hausdorff 0 .

Demonstração. Comecemos demonstrando que o espectro essencial de $J_{\delta, \phi}^{j}$ é inteiramente singular-contínuo. De acordo com o Teorema F.2.17, é suficiente mostrar que $\sum_{k=n}^{\infty}\left\|T_{j}(k ; \lambda)\right\|^{-2}=\infty$, para todo $\lambda \in I_{j}$, a menos de um conjunto de medida de Lebesgue nula $A_{j}$ (vide a demonstração do Teorema 3.3.3 para maiores detalhes). Do Lema 3.1.11, da identidade (3.1.18), e das desigualdades (5.2.4) e (5.2.5), segue que

$$
\sum_{k=n}^{\infty}\left\|T_{j}(k ; \lambda)\right\|^{-2} \geq \tilde{C}_{j}^{-2} \sum_{k=n}^{\infty} e^{c k^{\gamma}} \prod_{l=0}^{k} q_{l}^{2} \sin ^{2 k} \varphi_{j} \geq \tilde{C}_{j}^{-2} \sum_{k=n}^{\infty} e^{c_{2} k^{\delta}} \sin ^{2 k} \varphi_{j}
$$

diverge para $\varphi_{j}=1 / 2 \arccos (\lambda-2 \cos (2 \pi j / L)) \neq 0, \pi$ e para todo $\omega \in \Gamma=\underset{n=1}{\times}\{-n, \ldots, n\}$ com respeito a $\nu$, o que demonstra nossa afirmação.

Seguimos agora os mesmos passos empregados na demonstração do Teorema 4.1.3; temos para $a_{m}<l \leq a_{m+1}$ a estimativa

$$
\begin{aligned}
\sum_{n=0}^{l}\left\|T_{j}(n ; \lambda)\right\|^{2} & \leq C_{j}^{2} \sum_{k=1}^{m} e^{c k^{\gamma}} \prod_{n=0}^{k} q_{n}^{-2}\left(\sin \varphi_{j}\right)^{-2 k}+C_{j}^{2}\left(l-a_{m}\right) \prod_{n=0}^{m} q_{n}^{-2}\left(\sin \varphi_{j}\right)^{-2 m} \\
& \leq C_{j}^{2} \sum_{k=1}^{m} e^{2 c k^{\gamma}} e^{-c_{2} k^{\delta}}\left(\sin \varphi_{j}\right)^{-2 k}+C_{j}^{2}\left(l-a_{m}\right) e^{c m^{\gamma}} e^{-c_{2} m^{\delta}}\left(\sin \varphi_{j}\right)^{-2 m} \\
& \leq C_{j}^{\prime}\left(\sum_{k=0}^{m} e^{2 c k^{\gamma}}+\left(l-a_{m}\right) e^{c m^{\gamma}}\right) \leq C_{j}^{\prime \prime} l^{2}
\end{aligned}
$$

o que implica, pelo Corolário 4.2.3, que a restrição $\mu_{j}\left(\left(I_{j} \backslash A_{j}\right) \cap \cdot\right)$ é $\alpha$-contínua somente se $\alpha=0$. Uma vez que, pela Proposição 4.1.1, a solução subordinada da equação de Schrödinger (3.4) (que satisfaz a condição de contorno $\phi), u_{j}^{\text {sub }}(\lambda)$, decai tão rápido quanto a norma $\left\|T_{j}(n ; \lambda)\right\|$ cresce (vale ressaltar que as hipóteses da Proposição 4.1.1, bem como sua demonstração, devem ser ligeiramente alteradas: por exemplo, apesar de $s_{n}$ não ser limitada, a desigualdade (3.3.5) continua válida, o que nos permite chegar às mesmas conclusões), obtemos

$$
\left\|u_{j}^{\mathrm{sub}}\right\|_{l_{j}}^{2} \leq C_{j}^{-2} \sum_{n=0}^{l_{j}}\left\|T_{j}(n ; \lambda)\right\|^{-2} \leq C_{j}^{-2} \sum_{k=0}^{m+1} e^{c_{1} k^{\delta}} \sin ^{2 k} \varphi_{j} \leq C_{j}^{\prime} e^{c_{1} m^{\delta}}
$$

para alguma constante finita $C_{j}^{\prime}$. 
Como $l^{\alpha} \geq e^{c \alpha m^{\gamma}}$ e $\gamma>\delta$, obtemos $\liminf _{l_{j} \rightarrow \infty}\left\|u_{j}^{\text {sub }}\right\|_{l_{j}}^{2} / l_{j}^{\alpha}=0$ para qualquer $\alpha>0$, e assim, segue do Corolário H.16 que a restrição $\mu_{j}\left(\left(I_{j} \backslash A_{j}\right) \cap \cdot\right)$ é 0-singular.

Por fim, temos do Teorema 4.1 .4 que $\mu_{j}\left(A_{j}\right)=0$ para quase todo $\phi$ com respeito à medida de Lebesgue (vide a demonstração do Teorema 4.1.3), e assim para quase todo $\phi$, a restrição $\mu_{j}\left(I_{j} \cap \cdot\right)$ possui dimensão Hausdorff 0 . Isto conclui a demonstração do teorema.

Observação 5.2.6 1. Segue das desigualdades (5.2.6) e (5.2.7), com $\delta \equiv 1$, que a medida espectral restrita a $\left\{\lambda \in I_{j}:\left(1-\lambda^{2} / 4\right) e^{c_{2}}>1\right\} \backslash A_{j}$ é singular-contínua com dimensão Hausdorff 0, e puramente pontual quando restrita a $\left\{\lambda \in I_{j}:\left(1-\lambda^{2} / 4\right) e^{c_{1}}<\right.$ $1\} \backslash A_{j}, A_{j}$ o conjunto de medida de Lebesgue nula definido na demonstração do Teorema 5.2.5.

2. Repare que $q_{k}^{-2}=e^{c \gamma k^{\gamma-1}-c_{1} \delta k^{\delta-1}}$, com $\gamma>\delta>1, \delta>\gamma-1$ e $c_{1}$ suficientemente grande, satisfaz a desigualdade (5.2.4), tendo em vista a estimativa

$$
n^{\kappa}=\kappa \int_{0}^{n} x^{\kappa-1} d x<\sum_{k=0}^{n} \kappa k^{\kappa-1}<\kappa \int_{0}^{n+1} x^{\kappa-1} d x=(n+1)^{\kappa}
$$

para $\kappa=\gamma$ e $\delta$, dada a desigualdade

$$
\gamma(n+1)^{\gamma-1} \geq(n+1)^{\gamma}-n^{\gamma}=\int_{n}^{n+1} \gamma x^{\gamma-1} d x \geq \gamma n^{\gamma-1} .
$$

Seguimos, mais uma vez, os mesmos argumentos que nos levam aos Teoremas 5.1.3 e 5.2 .3 a fim de obter o

Teorema 5.2.7 Seja $\mathcal{J}_{\delta, \phi}$ a matriz bloco-Jacobi definida por (2.1.1), associada ao operador de diferença finita $\Pi_{P, \phi}$ definido por (1.6) e que satisfaz as condições de contorno (1.16), (1.17), com $P$ dado por (5.2.3) e sujeito à condição de esparsidade (5.1) $(\gamma>1$ e c > 0). Então, para quase toda condição de contorno $\phi \in[0, \pi)$ e para todo $\omega \in \Gamma=$ $\underset{n=1}{\infty}\{-n, \ldots, n\}$ com respeito a $\nu$, a medida espectral $\Omega_{00}$ restrita ao intervalo definido por (2.4.3) é puramente singular-contínua e possui dimensão Hausdorff 0. 



\section{Capítulo 6}

\section{Conclusões e problemas em aberto}

Analisamos nesse trabalho uma possível extensão, a uma faixa $\Lambda \equiv \mathbb{Z}_{+} \times\{0,1, \ldots, L-1\}$ de tamanho $L$ no plano $\mathbb{Z}_{+}^{2}$, do modelo unidimensional com perturbações esparsas proposto por Marchetti et.al., representado pelo operador $\Delta_{P, \phi}$ (definido por (1.4)). O modelo que propomos é definido como uma soma direta de dois operadores de diferença finita, cada qual atuando em uma direção: o operador unidimensional $\Delta_{P, \phi}$ em $\mathbb{Z}_{+}$, e o laplaciano livre na direção perpendicular. Essa estrutura peculiar nos permitiu tratá-lo com técnicas bastante sofisticadas e exclusivas de operadores de Schrödinger esparsos unidimensionais.

Apesar de simples, o modelo constitui um primeiro passo na tentativa de se compreender a natureza espectral de operadores de Schrödinger em mais de uma dimensão. A motivação, como o discutido no Capítulo 1, é apresentar um modelo que exiba uma transição entre espectros absolutamente contínuo e puramente pontual, fisicamente associada a uma transição entre, por exemplo, as fases condutora e isolante em uma estrutura cristalina metálica com impurezas esparsas (ou seja, cujas distâncias entre si crescem rapidamente à medida que estas se afastam da origem).

Mostramos no Capítulo 2 que a medida espectral, de importância ímpar na caracterização de um operador auto-adjunto, é na realidade representada por uma matriz cujas entradas (nesse caso particular) nada mais são do que combinações lineares de translações da medida espectral da matriz de Jacobi $J_{\delta, \phi}$, a representação matricial do operador $\Delta_{\delta, \phi}$ (equação (2.2.6)). De fato, é possível mostrar que a medida espectral matricial, $\Omega$, é absolutamente contínua com respeito ao elemento $\Omega_{00}$ (isto é, $\Omega$ atribui peso nulo a conjuntos com $\Omega_{00}$-medida nula; este é o resultado da Proposição 2.3.2), que por sua vez, pode ser tratado como a "medida de mistura" $\frac{1}{L} \sum_{j=0}^{L-1} \mu_{j}, \mu_{j}$ a medida associada à matriz de Jacobi $J_{\delta, \phi}^{j}$ (a representação matricial do operador unidimensional "transladado" $\Delta_{\delta, \phi}^{j}$, definido por (3.4)). Desse modo, identificamos as principais características espectrais de $\Omega$ simplesmente estudando o comportamento coletivo das medidas $\mu_{l}$, justificando, em parte, a afirmação de que o operador $\Pi_{\delta, \phi}$ é essencialmente unidimensional.

Para tanto, combinamos as poderosas ferramentas desenvolvidas por Last e Simon (que relacionam o comportamento assintótico da norma da matriz de transferência aos tipos espectrais), presentes no Apêndice F, ao Teorema 2.3 de [KLS] (Teorema F.3.3) e 
às técnicas desenvolvidas por Marchetti et.al., que a partir da hipótese de distribuição uniforme dos ângulos de Prüfer (Hipótese 3.1.6) permitem a obtenção precisa do comportamento médio (ou seja, com "probabilidade 1"; vide o Lema 3.1.11) da seqüência dos raios de Prüfer. Demonstramos, dessa forma, a existência de uma transição entre os espectros (essenciais) singular-contínuo e puramente pontual (Teorema 3.3.3) das matrizes de Jacobi $J_{\delta, \phi}^{j}$. Mais ainda, mostramos, graças a uma otimização do Teorema F.2.15 de [LS1] para operadores esparsos (Teorema 3.3.1), que essa transição é aguda, isto é, não existe uma região de transição entre os tipos espectrais (aprimorando um resultado análogo presente em [MWGA]).

A hipótese de distribuição uniforme dos ângulos de Prüfer pode ser facilmente verificada para as matrizes de Jacobi $J_{\delta, \phi}^{j, \omega}$ (a menos de um conjunto de medida de Lebesgue nula), cujas posições das "perturbações" são, por hipótese, variáveis aleatórias independentes e uniformemente distribuídas, uma idéia de Zlatoš, que estudou em [Z] um modelo bastante similar ao de Marchetti et.al; esse é conteúdo do Teorema 3.2.5.

O conhecimento preciso do comportamento assintótico médio dos raios de Prüfer também se mostrou crucial na caracterização total da parte singular-contínua do espectro, possível graças ao seminal trabalho de Jitomirskaya e Last [JL], que relaciona propriedades locais de escala de algumas soluções especiais da equação de Schrödinger (3.4) (entenda por solução especial uma solução subordinada, um conceito originalmente proposto por Gilbert e Pearson em [GP] para o problema de Sturm-Liouville contínuo) a propriedades de continuidade e singularidade de medidas de Borel com respeito a medidas Hausdorff (Apêndices G e H). A saber, mostramos que, dada uma solução $v_{j}$ da equação de Schrödinger (3.4), cujo raio de Prüfer associado apresenta o comportamento médio descrito anteriormente (repare que isso ocorre com probabilidade 1), existe uma solução subordinada $u_{j}$, cujo raio de Prüfer decai com a mesma taxa de crescimento de $v_{j}$ (Proposição 4.1.1). Obtemos, portanto, a dimensão Hausdorff (um indicativo da "singularidade" de uma medida de Borel; quanto mais seu valor se aproxima de 1, mais a medida se assemelha à medida de Lebesgue) exata de cada medida espectral $\mu_{j}$ (Teorema 4.1.3); vale destacar que um resultado parecido com esse foi obtido por Zlatos em [Z] justamente para operadores com uma incerteza na posição das perturbações.

Em seguida, estendemos o Teorema 1.2 de [JL] e seus subseqüentes corolários para a medida de mistura $\Omega_{00}$ (Teorema 4.2.1 e Corolários 4.2.3, 4.2.4 e 4.2.5), já que estes determinam somente o comportamento individual de cada medida $\mu_{j}$. Essa extensão nos permitiu obter com exatidão (a menos de um conjunto de medida de Lebesgue nula e para quase toda realização de $\omega$ ) a dimensão Hausdorff de $\Omega_{00}$, e conseqüentemente da medida espectral matricial $\Omega$ (Teorema 4.2.6). Pelo que sabemos, este é o primeiro trabalho (inspirado em $[\mathrm{CMW}]$ ) onde se obtém explicitamente a dimensão Hausdorff da medida espectral associada a uma classe de operadores bloco-Jacobi.

É interessante salientar mais uma vez o comportamento peculiar da medida $\Omega_{00}$, que sempre herda o comportamento mais singular entre seus componentes unidimensionais (o resultado é conseqüência direta do Teorema 4.2.1 e da definição adotada de singularidade 
e continuidade de uma mistura de medidas). Não foi possível, no entanto, determinar a multiplicidade de $\Omega$, já que este resultado depende diretamente de estimativas, que desconhecemos, das partes reais das funções $m_{j}$ de Weyl-Titchmarsh (vide a equação (2.3.5) para maiores esclarecimentos).

Por fim, estudamos no Capítulo 5 os mesmos operadores definidos em $\Lambda$ com condições de esparsidade sub e super-geométricas (ou seja, a distância entre as perturbações cresce ora sub-geometricamente, ora super-geometricamente, com respeito à origem). Para uma esparsidade sub-geométrica, constatamos que o espectro essencial de $\mathcal{J}_{\delta, \phi}$ é puramente pontual para quase toda condição de contorno $\phi$ (vide a demonstração do Teorema 5.1.1) e quase todo $\omega$ (Teorema 5.1.3). Em seguida, analisamos duas situações distintas de esparsidade super-geométrica: na primeira, onde as perturbações são constantes (vide (1.9)), demonstramos (Teorema 5.2.3) que a medida espectral matricial associada a $\mathcal{J}_{\delta, \phi}$ é puramente singular-contínua, com dimensão Hausdorff igual a 1 (sendo portanto, um exemplo do caso limítrofe entre medidas singulares e contínuas com respeito à medida de Lebesgue; vide o item 1 da Observação G.11); na segunda, onde a intensidade das perturbações passa a variar com suas posições, demonstramos (Teorema 5.2.5) que, para quase toda condição de contorno $\phi$ e para toda realização de $\omega$, a medida $\Omega$ é também puramente singular-contínua com dimensão igual a zero (sendo agora um exemplo do caso limítrofe entre medidas contínuas e puramente pontuais; vide novamente o item 1 da Observação G.11). Podemos ainda mostrar (vide o ítem 1 da Observação 5.2.6) que, para certas condições dos parâmetros, este último modelo exibe uma transição entre os espectros singular-contínuo e puramente pontual, cuja medida espectral associada possui dimensão Hausdorff 0; isso, em certo sentido, reforça a idéia de que a demonstração do Teorema 6.1 de $[\mathrm{Z}]$ está incompleta (vide o ítem 3 da Observação 3.3.4).

Nosso próximo passo é, como discutido no Capítulo 1, estender o modelo da faixa $\Lambda$ ao plano $\mathbb{Z}_{+}^{2}$. Uma extensão não trivial é dada pela definição (1.19), em que as perturbações agora ocorrem em ambas direções. As técnicas de diagonalização empregadas no estudo do operador $\Pi_{\delta, \phi}$ deixam de ser válidas nessa situação mais geral. Devemos, portanto, recorrer a outras técnicas.

De fato, a Proposição 2.3.10 se torna particularmente útil nesse sentido, já que sua principal idéia continua válida. A saber, podemos representar $\Omega$ como a produto de convolução

$$
\Omega(B)=\left(\mu_{\psi_{1}}^{1} * \mu_{\psi_{2}}^{2}\right)(B)
$$

em que $\mu_{\psi_{1}}^{1}$ e $\mu_{\psi_{2}}^{2}$ designam, respectivamente, as medidas espectrais associadas às matrizes de Jacobi $J_{P_{1(2)}, \phi_{1(2)}}$ e aos vetores (unimodulares e cíclicos) $\psi_{1}$ e $\psi_{2}$.

Como discutido no Capítulo 1, o fato das medidas $\mu_{\psi_{1}}^{1}$ e $\mu_{\psi_{2}}^{2}$ serem singulares para a classe de matrizes de Jacobi que consideramos (isto é, com as seqüências $P_{1(2)}$ satisfazendo as relações (1.9)-(1.11)) torna possível a existência de uma transição entre os espectros (essenciais) absolutamente contínuo e puramente pontual. A nossa idéia é obter, com precisão, o comportamento assintótico das transformadas de Fourier das medidas $\mu_{\psi_{1}}^{1}$ e 
$\mu_{\psi_{2}}^{2}\left(\hat{\mu}_{\psi_{1}}^{1}(t)\right.$ e $\hat{\mu}_{\psi_{2}}^{2}(t)$, respectivamente), e a partir daí usar o Teorema da convolução e obter

$$
\hat{\mu}_{\psi}(t)=\hat{\mu}_{\psi_{1}}^{1}(t) \hat{\mu}_{\psi_{2}}^{2}(t)
$$

onde $\psi=\psi_{1} \otimes \psi_{2}$ (vide o Capítulo 1 para uma discussão completa).

Os poucos trabalhos [KR, Si1] que se propõem a analisar o comportamento assintótico das transformadas de Fourier das medidas espectrais de operadores de Schrödinger esparsos apresentam técnicas de resolução bastante específicas à situação de esparsidade supergeométrica, e portanto não de aplicam aos modelos estudados aqui. Tanto Remling e Krutikov, em [KR], quanto Simon, em [Si1], obtiveram estimativas da ordem $\left|\hat{\mu}_{\psi}(t)\right| \sim t^{-1 / 2}$, no limiar do espectro singular-contínuo (vide o critério (1.20)).

Nossa idéia é tentar conectar de alguma forma esse comportamento assintótico (conhecido como dimensão de Fourier; vide $[\mathrm{M}]$ ) à dimensão Hausdorff da medida, obtida com exatidão. Infelizmente, até o presente momento, não fomos capazes de determinar essa conexão, e assim este permacene um problema em aberto.

Por fim, como possíveis trabalhos a serem desenvolvidos, podemos tentar aplicar as técnicas aqui apresentadas a operadores de Schrödinger definidos em estruturas mais gerais, como por exemplo, árvores de Cayley. Acreditamos ser possível demonstrar a existência da almejada transição do espectro absolutamente contínuo ao puramente pontual para modelos diferentes do estudado por Klein em $[\mathrm{K}]$. O trabalho de Froese et. al. [FHS1] é um bom indicativo da existência de espectro absolutamente contínuo para potenciais menos restritos (são transversalmente periódicos e radialmente $l^{\infty}$ ).

$\mathrm{Na}$ direção oposta se encontram os trabalhos de Breuer [Bre, Bre1]: estes apresentam exemplos de grafos onde o operador de Schrödinger discreto apresenta uma medida espectral puramente singular, infinitamente degenerada. No caso, as árvores consideradas possuem ramificações distribuídas esparsamente, de tal modo que o laplaciano discreto $\Delta$ é

$$
\Delta \cong \oplus_{l=0}^{\infty}\left(J_{l} \oplus J_{l} \oplus \cdots \oplus J_{l}\right)
$$

onde $J_{l}$ são matrizes de Jacobi com entradas nas primeiras diagonais que satisfazem

$$
p_{n}= \begin{cases}\sqrt{2} & \text { se } \quad j=a_{k} \in \mathcal{A}, k>l \\ 1 & \text { de outra forma }\end{cases}
$$

para o caso de uma árvore binária com bifurcações esparsas dadas por (1.11). A degenerescência do $l$-ésimo subespaço onde se decompõe o laplaciano é $2^{l}-2^{l-1}$.

Obtendo-se a decomposição acima, basta aplicar todas as técnicas que possuímos para uma descrição detalhada do espectro (Breuer usa os resultados de [Z] em sua análise; este é essencialmente o conteúdo do trabalho [Bre1]). Os resultados do Teorema 4.2.6 se estendem quase completamente a este problema: as diferenças envolvem o intervalo $I$, que agora deve ser restrito à intersecção $I^{(j)}=(-2,2) \bigcap B^{(j)}$ (o espectro essencial contém 
o intervalo $(-2,2)$; vide o Teorema 3.8 de [Bre1]), $j=2^{l}-2^{l-1}$, e a dimensão espectral local de $\Omega$, que é simplesmente

$$
\alpha_{\Omega}(\lambda)=1-\frac{\ln r}{\ln \beta}
$$

$\lambda \in I^{(j)}$, com $r=r(\sqrt{2}, \lambda)$ dado por (3.1.30) (compare o resultado acima com a equação (4.3) de [Bre1]).

Seria, portanto, interessante tratar modelos nessas árvores que eventualmente conseguissem mesclar características dos modelos descritos acima. 



\section{Apêndice A}

\section{Propriedades espectrais de operadores limitados}

Apresentamos neste apêndice as principais propriedades espectrais dos operadores lineares limitados definidos em um espaço de Hilbert arbitrário, objetos de estudo desse trabalho.

Começamos pelas seguintes definições.

Definição A.1 Seja $T: \mathcal{H} \longrightarrow \mathcal{H}$ um operador linear definido no espaço de Hilbert $\mathcal{H}$. $T$ é dito limitado se existe um número real c tal que, para todo $x \in \mathcal{H}$,

$$
\|T x\| \leq c\|x\| \text {. }
$$

Denotamos por $B(\mathcal{H})$ o conjunto dos operadores lineares limitados no espaço de Hilbert $\mathcal{H}$.

Definição A.2 Seja $T: \mathcal{H}_{1} \longrightarrow \mathcal{H}_{2}$ um operador linear limitado, onde $\mathcal{H}_{1}$ e $\mathcal{H}_{2}$ são espaços de Hilbert. Então, o operador Hilbert-adjunto $T^{*}$ de $T$ é o operador

$$
T^{*}: \mathcal{H}_{2} \longrightarrow \mathcal{H}_{1}
$$

tal que, para todos $x \in \mathcal{H}_{1}$ e $y \in \mathcal{H}_{2}$,

$$
\langle T x, y\rangle=\left\langle x, T^{*} y\right\rangle
$$

Mostremos que o operador $T^{*}$ definido acima existe.

Teorema A.3 O operador Hilbert-adjunto $T^{*}$ de $T$, como apresentado na Definição A.2, existe, é único e é um operador linear limitado com norma

$$
\left\|T^{*}\right\|=\|T\|
$$

Demonstração. A função

$$
h(y, x)=\langle y, T x\rangle
$$


define uma forma sesquilinear no espaço produto $\mathcal{H}_{2} \times \mathcal{H}_{1}$, uma vez que o produto interno é sesquilinear e $T$ é linear. De fato, a sesquilinearidade da forma (A.3) é vista de

$$
h\left(y, a x_{1}+b x_{2}\right)=\left\langle y, T\left(a x_{1}+b x_{2}\right)\right\rangle=\bar{a}\left\langle y, T x_{1}\right\rangle+\bar{b}\left\langle y, T x_{2}\right\rangle=\bar{a} h\left(y, x_{1}\right)+\bar{b} h\left(y, x_{2}\right) .
$$

A forma $h$ é limitada, já que obtemos, da desigualdade de Cauchy-Schwarz,

$$
|h(y, x)|=|\langle y, T x\rangle| \leq\|y\|\|T x\| \leq\|T\|\|x\|\|y\| .
$$

Isso também implica que $\|h\| \leq\|T\|$, onde definimos a norma

$$
\|h\|=\sup _{x, y \neq 0} \frac{|\langle y, T x\rangle|}{\|x\|\|y\|} .
$$

Mais ainda, temos que $\|h\| \geq\|T\|$ graças a

$$
\|h\|=\sup _{x, y \neq 0} \frac{|\langle y, T x\rangle|}{\|x\|\|y\|} \geq \sup _{x, T x \neq 0} \frac{|\langle T x, T x\rangle|}{\|x\|\|T x\|}=\|T\| .
$$

Juntos, concluímos que

$$
\|h\|=\|T\| .
$$

Obtemos, do Teorema de representação de Riesz (Teorema 3.8-4 de [Kre]),

$$
h(y, x)=\left\langle T^{*} y, x\right\rangle
$$

segue do mesmo teorema que $T^{*}: \mathcal{H}_{2} \longrightarrow \mathcal{H}_{1}$ é um operador linear limitado e unicamente determinado, com norma

$$
\left\|T^{*}\right\|=\|h\|=\|T\| .
$$

Isto demonstra (A.2). Temos também a identidade $\langle y, T x\rangle=\left\langle T^{*} y, x\right\rangle$, obtida através da comparação entre (A.3) e (A.4), de modo que chegamos a (A.1) conjugando a expressão anterior. Isso demonstra o teorema.

Definição A.4 (Operador auto-adjunto). Um operador linear limitado $T: \mathcal{H} \longrightarrow \mathcal{H}$ definido em um espaço de Hilbert $\mathcal{H}$ é dito auto-adjunto se $T^{*}=T$, onde $T^{*}$ é o operador Hilbert-adjunto presente na Definição A.2. Nesse caso, a fórmula (A.1) se torna

$$
\langle T x, y\rangle=\langle x, T y\rangle
$$

Existe um critério bastante simples que nos permite determinar se um dado operador é auto-adjunto.

Teorema A.5 Seja $T: \mathcal{H} \longrightarrow \mathcal{H}$ um operador linear limitado em um espaço de Hilbert H. Então:

(i) Se T é auto-adjunto, $\langle T x, x\rangle$ é um número real para todo $x \in \mathcal{H}$. 
(ii) Se $\mathcal{H}$ é complexo e $\langle T x, x\rangle$ é real para todo $x \in \mathcal{H}$, então o operador $T$ é auto-adjunto. Demonstração. (i) Se $T$ é auto-adjunto, então para todo $x$,

$$
\overline{\langle T x, x\rangle}=\langle x, T x\rangle=\langle T x, x\rangle \text {. }
$$

Logo, $\langle T x, x\rangle$ é igual a seu complexo conjungado, sendo portanto real.

(ii) Se $\langle T x, x\rangle$ é real para todo $x$, então

$$
\langle T x, x\rangle=\overline{\langle T x, x\rangle}=\overline{\left\langle x, T^{*} x\right\rangle}=\left\langle T^{*} x, x\right\rangle \text {. }
$$

Assim,

$$
0=\langle T x, x\rangle-\left\langle T^{*} x, x\right\rangle=\left\langle\left(T-T^{*}\right) x, x\right\rangle
$$

e $T-T^{*}=0$, o que conclui a demonstração.

Observação A.6 É essencial, na parte (ii) do teorema, que $\mathcal{H}$ seja complexo. Isso é claro, uma vez que para $\mathcal{H}$ real, o produto interno é real, o que torna $\langle$ Tx, x〉 real sem qualquer hipótese adicional com respeito ao operador linear $T$.

\section{A.1 Espectro}

Se $T$ é um operador linear definido em $\mathbb{C}^{n}$, então os autovalores de $T$ consistem no conjunto dos números complexos $\lambda$ tais que o determinante do operador $T-\lambda I$ é igual a zero. Denominamos tal conjunto de pontos por espectro de $T$. Este possui apenas $n$ pontos, já que $\operatorname{det}(T-\lambda I)$ é um polinômio de grau $n^{\dagger}$. Se $\lambda$ não é um autovalor, então $T-\lambda I$ possui uma inversa, uma vez que $\operatorname{det}(T-\lambda I) \neq 0$.

A teoria espectral de operadores definidos em espaços de dimensão infinita (como os espaços de Hilbert, por exemplo), é bem mais rica em detalhes e muito importante na compreensão dos operadores em si.

Definição A.1.1 (Definição 7.2-1 de [Kre]) Seja $X \neq\{0\}$ um espaço complexo normado e $T: \mathcal{D}(T) \longrightarrow X$ um operador linear com domínio $\mathcal{D}(T) \subset X$. Dizemos que o número complexo $\lambda$ pertence ao conjunto resolvente, $\rho(T)$, de $T$ se

(a) $R_{\lambda}(T):=(T-\lambda I)^{-1}$ existe,

(b) $R_{\lambda}(T)$ é limitado,

(c) $R_{\lambda}(T)$ é definido em um conjunto denso em $X$,

${ }^{\dagger} \mathrm{O}$ Teorema fundamental da álgebra garante a existência de $n$ raízes $\operatorname{de} \operatorname{det}(T-\lambda I)$, não necessariamente distintas. 
onde $R_{\lambda}(T)$ é o chamado resolvente do operador $T$. Se $\lambda \notin \rho(T)$, dizemos que $\lambda$ pertence ao espectro, $\sigma(T)$, de $T$. Mais ainda, o espectro $\sigma(T)$ pode ser particionado em três conjuntos disjuntos:

$O$ espectro discreto, $\sigma_{\mathrm{d}}(T)$, é o subconjunto de $\sigma(T)$ tal que $R_{\lambda}(T)$ não existe. Um $\lambda \in \sigma_{\mathrm{d}}(T)$ é chamado de autovalor de $T$.

$O$ espectro contínuo, $\sigma_{\mathrm{c}}(T)$, é definido como o conjunto de pontos tais que $R_{\lambda}(T)$ satisfaz (a) e (c), porém é ilimitado.

$O$ espectro residual, $\sigma_{\mathrm{r}}(T)$, é definido como o conjunto de pontos tais que $R_{\lambda}(T)$ satisfaz (a) (podendo ser limitado ou não), porém não satisfaz (c), isto é, o domínio de $R_{\lambda}(T)$ não é denso em $X$.

Se o resolvente $R_{\lambda}(T)$ existe, este é linear pelo Teorema 2.6-10 de [Kre], que também mostra que $R_{\lambda}(T): \mathcal{R}(T-\lambda I) \longrightarrow \mathcal{D}(T-\lambda I)^{\dagger}$ existe se, e somente se, $(T-\lambda I) x=0$ caso $x=0$.

Dessa forma, se $(T-\lambda I) x=0$ para algum $x \neq 0$, então $\lambda \in \sigma_{\mathrm{d}}(T)$ por definição, isto é, $\lambda$ é um autovalor de $T$ (repare que isto concorda com a definição de autovalor para operadores definidos em um espaço de dimensão finita). O vetor $x$ é chamado de autovetor (em um sentido generalizado) de $T$.

Restrinjamos nosso estudo aos operadores lineares limitados. Comecemos pelo importante

Teorema A.1.2 (Teorema 7.3-1 de [Kre]) Seja X um espaço de Banach (isto é, um espaço normado completo) e $T: X \longrightarrow X$ um operador linear e limitado. Se $\|T\|<1$, então $(I-T)^{-1}$ existe como um operador linear e limitado em todo espaço $X$, de modo que

$$
(I-T)^{-1}=\sum_{j=0}^{\infty} T^{j}=I+T+T^{2}+\cdots
$$

(a convergência é no sentido da norma $\|\cdot\|$ definida nesse espaço).

Demonstração. Temos que $\left\|T^{j}\right\| \leq\|T\|^{j}$, uma vez que $T$ é um operador limitado, de modo que a série presente no membro direito de (A.1.1) converge absolutamente caso $\|T\|<1$. Uma vez que $X$ é completo, a convergência absoluta da série implica convergência no sentido forte (isto é, em norma).

Denotemos a série em (A.1.1) por $S$. Resta-nos mostrar que $S=(I-T)^{-1}$. Para tanto, calculamos

$$
(I-T)\left(I+T+\cdots T^{n}\right)=\left(I+T+\cdots T^{n}\right)(I-T)=I-T^{n+1} .
$$

Como $\lim _{n \rightarrow \infty} T^{n+1}=0$ (já que $\|T\|<1$ ), concluímos da identidade acima que

$$
(I-T) S=S(I-T)=I
$$

\footnotetext{
${ }^{\dagger} \mathcal{R}(T-\lambda I)$ é o conjunto imagem do operador $T-\lambda I$
} 
e que portanto, $S=(I-T)^{-1}$, pela definição de operador inverso. Isso conclui a demonstração.

Como primeira aplicação do Teorema A.1.2 temos o

Teorema A.1.3 (Teorema 7.3-2 de [Kre]) $O$ conjunto resolvente $\rho(T)$ de um operador linear limitado $T$ em um espaço de Banach $X$ é aberto; logo, seu espectro é fechado.

Demonstração. Se $\rho(T)=\emptyset$, então $\rho(T)$ é aberto. Suponhamos então que $\rho(T) \neq \emptyset$. Para um dado $\lambda_{0} \in \rho(T)$ e para qualquer $\lambda \in \mathbb{C}$ temos a identidade

$$
T-\lambda I=T-\lambda_{0} I-\left(\lambda-\lambda_{0}\right) I=\left(T-\lambda_{0} I\right)\left[I-\left(\lambda-\lambda_{0}\right)\left(T-\lambda_{0} I\right)^{-1}\right],
$$

que pode ser reescrita como

$$
T_{\lambda}=T_{\lambda_{0}} V
$$

onde $V=I-\left(\lambda-\lambda_{0}\right) R_{\lambda_{0}}$. Uma vez que $\lambda_{0} \in \rho(T)$ e $T$ é limitado, o Lema 7.2-3(b) de [Kre] nos diz que o resolvente $R_{\lambda_{0}}=T_{\lambda_{0}}^{-1}$ é também um operador linear e limitado em $X$. Além disso, o Teorema A.1.2 mostra que $V$ possui uma inversa

$$
V^{-1}=\sum_{j=0}^{\infty}\left[\left(\lambda-\lambda_{0}\right) R_{\lambda_{0}}\right]^{j}=\sum_{j=0}^{\infty}\left(\lambda-\lambda_{0}\right)^{j} R_{\lambda_{0}}^{j}
$$

que também é linear e limitada para todo $\lambda$ tal que $\left\|\left(\lambda-\lambda_{0}\right) R_{\lambda_{0}}\right\|<1$, isto é, caso

$$
\left|\lambda-\lambda_{0}\right|<\left\|R_{\lambda_{0}}\right\|^{-1}
$$

Como $R_{\lambda_{0}}$ é linear e limitado, concluímos deste fato e da relação (A.1.2) que, para todo $\lambda$ satisfazendo (A.1.3), o operador $T_{\lambda}$ possui uma inversa

$$
R_{\lambda}=T_{\lambda}^{-1}=\left(T_{\lambda_{0}} V\right)^{-1}=V^{-1} R_{\lambda_{0}}
$$

Logo, (A.1.3) representa uma vizinhança de $\lambda_{0}$ pertencente ao conjunto resolvente. Como $\lambda_{0} \in \rho(T)$ foi escolhido arbitrariamente, concluímos que $\rho(T)$ é aberto, e que portanto, seu complemento $\sigma(T)=\mathbb{C} \backslash \rho(T)$ é fechado.

Teorema A.1.4 (Teorema 7.3-4 [Kre]) O espectro $\sigma(T)$ de um operador linear limitado $T: X \longrightarrow X$ em um espaço de Banach complexo é compacto e está contido no disco dado por

$$
|\lambda| \leq\|T\|
$$

Desse modo, o conjunto resolvente $\rho(T)$ de $T$ não é vazio. 
Demonstração. Seja $\lambda \neq 0$. Obtemos do Teorema A.1.2 a representação

$$
R_{\lambda}=-\frac{1}{\lambda}(I-\kappa T)^{-1}=-\frac{1}{\lambda} \sum_{j=0}^{\infty}\left(\frac{1}{\lambda} T\right)^{j}
$$

de modo que, pelo mesmo teorema, a série converge para todo $|\lambda|>\|T\|$. Concluímos, portanto, que $\sigma(T)$ pertence ao disco (A.1.4); conseqüentemente, $\sigma(T)$ é limitado. Mais ainda, $\sigma(T)$ é fechado, pelo Teorema A.1.3, e portanto compacto.

Outras propriedades básicas do resolvente são expressas pelo

Teorema A.1.5 (Teorema 7.4-1 [Kre]) Seja $X$ um espaço de Banach complexo, $T$ um operador linear limitado $T: X \longrightarrow X$ e $\lambda, \mu \in \rho(T)$. Então

(a) O resolvente $R_{\lambda}$ satisfaz a relação de Hilbert ou primeira identidade do resolvente

$$
R_{\mu}-R_{\lambda}=(\mu-\lambda) R_{\mu} R_{\lambda}
$$

(b) $R_{\lambda}$ comuta com qualquer operador $S$ linear e limitado, definido em $X$, que comute com $T$.

(c) Temos

$$
R_{\lambda} R_{\mu}=R_{\mu} R_{\lambda}
$$

Demonstração. (a) Segue do Lema 7.2-3 de [Kre] que a imagem de $T_{\lambda}$ é inteiramente contida em $X$. Logo, $I=T_{\lambda} R_{\lambda}=R_{\lambda} T_{\lambda}$, e portanto

$$
R_{\mu}-R_{\lambda}=R_{\mu}\left(T_{\lambda} R_{\lambda}\right)-\left(R_{\mu} T_{\mu}\right) R_{\lambda}=R_{\mu}[T-\lambda I-(T-\mu I)] R_{\lambda}=(\mu-\lambda) R_{\mu} R_{\lambda}
$$

(b) Por hipótese, $S T=T S$. Logo $S T_{\lambda}=T_{\lambda} S$, e assim

$$
R_{\lambda} S=R_{\lambda} S T_{\lambda} R_{\lambda}=R_{\lambda} T_{\lambda} S R_{\lambda}=S R_{\lambda}
$$
$R_{\mu}$.

(c) $R_{\mu}$ comuta com $T$ por (b), o que implica pelo mesmo ítem que $R_{\lambda}$ comuta com

Passemos finalmente ao estudo das principais propriedades espectrais dos operadores lineares limitados auto-adjuntos em um espaço de Hilbert. Comecemos caracterizando os autovalores e autovetores (caso existam) desse tipo de operadores.

Teorema A.1.6 (Teorema 9.1-1 de $[\mathbf{K R}]$ ) Seja $T: \mathcal{H} \longrightarrow \mathcal{H}$ um operador linear limitado auto-adjunto em um espaço de Hilbert complexo $\mathcal{H}$. Então:

(a) Todos os autovalores de $T$ (se existirem) são reais. 
(b) Autovetores que correspondam a autovalores de $T$ numericamente diferentes são ortogonais.

Demonstração. (a) Seja $\lambda$ um autovalor de $T$ e $x$ seu autovetor correspondente. Então $x \neq 0$ e $T x=\lambda x$. Concluímos do fato de $T$ ser auto-adjunto que

$$
\lambda\langle x, x\rangle=\langle\lambda x, x\rangle=\langle T x, x\rangle=\langle x, T x\rangle=\langle x, \lambda x\rangle=\bar{\lambda}\langle x, x\rangle .
$$

Como $\langle x, x\rangle=\|x\|^{2} \neq 0$ já que $x \neq 0$, concluímos que $\lambda=\bar{\lambda}$, e que portanto, $\lambda$ é real.

(b) Sejam $\lambda$ e $\mu$ autovalores de $T$, e sejam $x$ e $y$ seus autovetores correspondentes. Sendo $T$ auto-adjunto e $\mu$ real, obtemos

$$
\lambda\langle x, y\rangle=\langle\lambda x, y\rangle=\langle T x, y\rangle=\langle x, T y\rangle=\langle x, \mu x\rangle=\mu\langle x, y\rangle .
$$

Como $\lambda \neq \mu$, devemos ter $\langle x, y\rangle=0$, o que representa a ortogonalidade de $x$ e $y$.

Teorema A.1.7 (Teorema 9.1-2 de [Kre]) Seja $T: \mathcal{H} \longrightarrow \mathcal{H}$ um operador linear limitado auto-adjunto em um espaço de Hilbert complexo $\mathcal{H}$. Então, um número $\lambda$ pertence ao conjunto resolvente $\rho(T)$ de $T$ se, e somente se, existir um $c>0$ tal que, para todo $x \in \mathcal{H}$,

$$
\left\|T_{\lambda} x\right\| \geq c\|x\|
$$

Demonstração. Se $\lambda \in \rho(T)$, então $R_{\lambda}: \mathcal{H} \longrightarrow \mathcal{H}$ existe e é limitado, graças ao Lema 7.2-3 de [Kre]; digamos, $\left\|R_{\lambda}\right\|=k, k>0$. Como $I=R_{\lambda} T_{\lambda}$, segue para todo $x \in \mathcal{H}$ que

$$
\|x\| \leq\left\|R_{\lambda}\right\|\left\|T_{\lambda} x\right\|=k\left\|T_{\lambda} x\right\| .
$$

Logo, $\left\|T_{\lambda} x\right\| \geq c\|x\|$, com $c=1 / k$. Por outro lado, suponha a validade de (A.1.8), com $c>0$, para todo $x \in \mathcal{H}$. Provemos que:

(a) $T_{\lambda}: \mathcal{H} \longrightarrow T_{\lambda}(\mathcal{H})$ é uma aplicação bijetora;

(b) $T_{\lambda}(\mathcal{H})$ é um conjunto denso em $\mathcal{H}$;

(c) $T_{\lambda}(\mathcal{H})$ é um conjunto fechado em $\mathcal{H}$,

de modo que $T_{\lambda}(\mathcal{H})=\mathcal{H}$ e $R_{\lambda}$ é limitado pelo Teorema da inversa limitada (vide, por exemplo, o Teorema 4.12-2 de [Kre]).

(a) Mostremos que a identidade $T_{\lambda} x_{1}=T_{\lambda} x_{2}$ implica em $x_{1}=x_{2}$. Isto, no entanto, segue de (A.1.8), já que $T_{\lambda}$ é linear e

$$
0=\left\|T_{\lambda} x_{1}-T_{\lambda} x_{2}\right\|=\left\|T_{\lambda}\left(x_{1}-x_{2}\right)\right\| \geq c\left\|x_{1}-x_{2}\right\|
$$

como $\left\|x_{1}-x_{2}\right\|=0$, concluímos que $T_{\lambda}: \mathcal{H} \longrightarrow T_{\lambda}(\mathcal{H})$ é bijetiva, já que $x_{1}, x_{2}$ são arbitrários. 
(b) Mostremos que $x_{0} \perp \overline{T_{\lambda}(\mathcal{H})}$ implica em $x_{0}=0$, de modo que $\overline{T_{\lambda}(\mathcal{H})}=\mathcal{H}$ pelo Teorema de projeção (vide, por exemplo, o Teorema 3.3-4 de [Kre]). Seja $x_{0} \perp \overline{T_{\lambda}(\mathcal{H})}$. Então $x_{0} \perp T_{\lambda}(\mathcal{H})$, de modo que, para todo $x \in \mathcal{H}$, temos

$$
0=\left\langle T_{\lambda} x, x_{0}\right\rangle=\left\langle T x, x_{0}\right\rangle-\lambda\left\langle x, x_{0}\right\rangle .
$$

Uma vez que $T$ é auto-adjunto, obtemos

$$
\left\langle x, T x_{0}\right\rangle=\left\langle T x, x_{0}\right\rangle=\left\langle x, \bar{\lambda} x_{0}\right\rangle,
$$

e assim $T x_{0}=\bar{\lambda} x_{0}$. Uma possível solução é $x_{0}=0$, e $x_{0} \neq 0$ é impossível, já que dessa forma, $\bar{\lambda}$ seria um autovalor de $T$; como $\bar{\lambda}=\lambda$ (vide o Teorema A.1.6), teríamos $T_{\lambda} x_{0}=0$, e assim a relação (A.1.8) nos levaria à contradição

$$
0=\left\|T_{\lambda} x_{0}\right\| \geq c\left\|x_{0}\right\|>0
$$

já que $c>0$. Concluímos, portanto, que $T_{\lambda}(\mathcal{H})$ é denso em $\mathcal{H}$.

(c) Demonstramos que $y \in \overline{T_{\lambda}(\mathcal{H})}$ implica $y \in T_{\lambda}(\mathcal{H})$, e assim $T_{\lambda}(\mathcal{H})$ é fechado, o que resulta em $T_{\lambda}(\mathcal{H})=\mathcal{H}$, pelo ítem (b). Seja $y \in \overline{T_{\lambda}(\mathcal{H})}$. Então, existe uma seqüência $\left(y_{n}\right) \in T_{\lambda}(\mathcal{H})$ que converge a $y$. Como $y_{n} \in T_{\lambda}(\mathcal{H})$, temos que $y_{n}=T_{\lambda} x_{n}$ para algum $x_{n} \in \mathcal{H}$. Segue da relação (A.1.8) que

$$
\left\|x_{n}-x_{m}\right\| \leq \frac{1}{c}\left\|T_{\lambda}\left(x_{n}-x_{m}\right)\right\|=\frac{1}{c}\left\|y_{n}-y_{m}\right\|,
$$

o que mostra que $\left(x_{n}\right)$ é uma seqüência de Cauchy, já que $\left(y_{n}\right)$ é uma seqüência convergente. $\mathcal{H}$ é completo, de modo que $\left(x_{n}\right)$ converge, digamos, para um $x \in \mathcal{H}$. Como $T_{\lambda}$ é contínuo, segue que $y_{n}=T_{\lambda} x_{n} \longrightarrow T_{\lambda} x$. Por definição, $T_{\lambda} x \in T_{\lambda}(\mathcal{H})$. Como o limite é único, $T_{\lambda} x=y$, e portanto $y \in T_{\lambda}(\mathcal{H})$, o que nos permite concluir que $T_{\lambda}(\mathcal{H})$ é fechado, já que $y \in \overline{T_{\lambda}(\mathcal{H})}$ foi escolhido arbitrariamente.

Temos, portanto, que $T_{\lambda}(\mathcal{H})=\mathcal{H}$. Isso implica que $R_{\lambda}$ é um operador limitado, definido em todo espaço $\mathcal{H}$. Logo, $\lambda \in \rho(T)$.

Obtemos do Teorema A.1.7 o

Teorema A.1.8 (Teorema 9.1-3 de [Kre]) O espectro $\sigma(T)$ de um operador linear limitado auto-adjunto $T: \mathcal{H} \longrightarrow \mathcal{H}$ em um espaço de Hilbert complexo $\mathcal{H}$ é real.

Demonstração. Mostramos, usando o Teorema A.1.7, que todo $\lambda=\alpha+i \beta(\alpha, \beta \in \mathbb{R})$, com $\beta \neq 0$, deve pertencer a $\rho(T)$, de modo que $\sigma(T) \subset \mathbb{R}$.

Temos, para todo $x \neq 0$,

$$
\left\langle T_{\lambda} x, x\right\rangle=\langle T x, x\rangle-\lambda\langle x, x\rangle
$$

e como $\langle x, x\rangle$ e $\langle T x, x\rangle$ são números reais (vide o Teorema A.5),

$$
\overline{\left\langle T_{\lambda} x, x\right\rangle}=\langle T x, x\rangle-\bar{\lambda}\langle x, x\rangle .
$$


Obtemos, subtraindo as duas equações acima,

$$
\overline{\left\langle T_{\lambda} x, x\right\rangle}-\left\langle T_{\lambda} x, x\right\rangle=(\lambda-\bar{\lambda})\langle x, x\rangle=2 i \beta\|x\|^{2} .
$$

O membro esquerdo da identidade acima é simplesmente $-2 i \Im\left\langle T_{\lambda} x, x\right\rangle$; dividindoa por 2, tomando os valores absolutos e aplicando a desigualdade de Cauchy-Schwarz, obtemos

$$
|\beta|\|x\|^{2}=\left|\Im\left\langle T_{\lambda} x, x\right\rangle\right| \leq\left|\left\langle T_{\lambda} x, x\right\rangle\right| \leq\left\|T_{\lambda} x\right\|\|x\|,
$$

e portanto $|\beta|\|x\| \leq\left\|T_{\lambda} x\right\|$. Se $\beta \neq 0$, concluímos do Teorema A.1.7 que $\lambda \in \rho(T)$. Assim, $\lambda$ deve ser real a fim de que pertença ao espectro. Isso encerra a demonstração.

Acabamos de demonstrar que o espectro de um operador linear limitado auto-adjunto deve ser um subconjunto da reta real. Fica bastante claro a partir do Teorema A.1.4 que $\sigma(T)$, além de estar contido na reta, deve ser um conjunto compacto. De fato, temos o

Teorema A.1.9 (Teorema 9.2-1 de [Kre]) O espectro $\sigma(T)$ de um operador linear limitado auto-adjunto $T: \mathcal{H} \longrightarrow \mathcal{H}$ em um espaço de Hilbert complexo $\mathcal{H}$ está contido no intervalo fechado $[m, M]$ da reta real, onde

$$
m=\inf _{\|x\|=1}\langle T x, x\rangle, \quad M=\sup _{\|x\|=1}\langle T x, x\rangle .
$$

Demonstração. Mostramos que qualquer número real $\lambda=M+c$, com $c>0$ pertence ao conjunto resolvente $\rho(T)$. Para todo $x \neq 0$ e $v=\|x\|^{-1} x$, temos

$$
\langle T x, x\rangle=\|x\|^{2}\langle T v, v\rangle \leq\|x\|^{2} \sup _{\|\tilde{v}\|=1}\langle T \tilde{v}, \tilde{v}\rangle=\|x\|^{2} M
$$

Assim, $-\langle T x, x\rangle \geq-\|x\|^{2} M$, e pela desigualdade de Cauchy-Schwarz obtemos

$$
\left\|T_{\lambda} x\right\|\|x\| \geq-\langle T x, x\rangle+\lambda\langle x, x\rangle \geq(-M+\lambda)\|x\|^{2}=c\|x\|^{2},
$$

o que resulta em $\left\|T_{\lambda} x\right\| \geq c\|x\|$. Logo, segue do Teorema A.1.7 que $\lambda \in \rho(T)$. Para $\lambda<m$, a idéia da demonstração é a mesma.

Por fim, justificamos o porquê de distinguirmos o espectro residual dos demais tipos espectrais na Definição A.1.1.

Teorema A.1.10 (Teorema 9.2-4 de [Kre]) O espectro residual, $\sigma_{r}(T)$, de um operador linear limitado auto-adjunto $T: \mathcal{H} \longrightarrow \mathcal{H}$ em um espaço de Hilbert complexo $\mathcal{H}$ é vazio.

Demonstração. Mostramos que a hipótese $\sigma_{\mathrm{r}}(T) \neq \emptyset$ nos leva a uma contradição. Seja $\lambda \in \sigma_{\mathrm{r}}(T)$. Pela Definição A.1.1 de espectro residual, o operador $R_{\lambda}$ existe, no entanto 
seu domínio $\mathcal{D}\left(R_{\lambda}\right)$ não é denso em $\mathcal{H}$. Desse modo, existe um $y \neq 0$ em $\mathcal{H}$ ortogonal a $\mathcal{D}\left(R_{\lambda}\right)$. Como $\mathcal{D}\left(R_{\lambda}\right)$ está contido na imagem de $T_{\lambda}$, concluímos que

$$
\left\langle T_{\lambda} x, y\right\rangle=0
$$

para todo $x \in \mathcal{H}$. Como $\lambda \in \mathbb{R}$ e $T$ é auto-adjunto, obtemos $\left\langle x, T_{\lambda} y\right\rangle=0$ para todo $x$. Tomando $x=T_{\lambda} y$, obtemos $\left\|T_{\lambda} y\right\|^{2}=0$, e portanto

$$
T_{\lambda} y=T y-\lambda y=0 .
$$

Uma vez que $y \neq 0$, isso mostra que $\lambda$ é um autovalor de $T$, o que contradiz $\lambda \in \sigma_{\mathrm{r}}(T)$. Logo, $\sigma_{\mathrm{r}}(T)=\emptyset$. 


\section{Apêndice B}

\section{Teorema espectral}

Existem várias formulações distintas do chamado Teorema espectral para operadores limitados auto-adjuntos, que são, em um dado sentido, equivalentes. É particularmente interessante a formulação do teorema que afirma serem todos os operadores limitados auto-adjuntos equivalentes a operadores de multiplicação. Isto significa que dado um operador limitado auto-adjunto em um espaço de Hilbert $\mathcal{H}$, podemos sempre achar uma medida $\mu$ em um espaço mensurável $M$, e um operador unitário $U: \mathcal{H} \longrightarrow L^{2}(M, d \mu)^{*}$ tal que

$$
\left(U T U^{-1} f\right)(x)=F(x) f(x),
$$

para alguma função $F$ real mensurável e limitada em $M$.

Esta é claramente uma generalização do teorema para operadores de dimensão finita, que afirma que qualquer matriz de ordem $n$ auto-adjunta pode ser diagonalizada; a saber, dado um operador auto-adjunto $T$, definido em um espaço complexo $V, n$-dimensional, existem um operador unitário $U: V \longrightarrow \mathbb{C}^{n}$ e números reais $\lambda_{1}, \ldots, \lambda_{n}$, tais que

$$
\left(U T U^{-1} f\right)_{i}=\lambda_{i} f_{i}
$$

para cada $f=\left(f_{1}, \ldots, f_{n}\right) \in \mathbb{C}^{n}$.

Na prática, $M$ é a união de cópias de $\mathbb{R}$ e $F$ é $x$, de modo que o cerne da demonstração do teorema envolve a construção de certas medidas. Vamos introduzi-las agora.

\section{B.1 Teorema espectral multiplicativo e a decomposi- ção das medidas espectrais}

Fixemos $T$, um operador limitado auto-adjunto em $\mathcal{H}$. Seja $\psi \in \mathcal{H}$. Então, uma vez que $f \longmapsto\langle f(T) \psi, \psi\rangle$ é um funcional linear positivo em $C(\sigma(T))^{\dagger}$, temos do Teorema de

${ }^{*} L^{2}(M, d \mu)$ representa o conjunto das classes de equivalência das funções mensuráveis em $M$ que satisfazem $\left(\int_{M}|f(x)|^{2} d \mu(x)\right)^{1 / 2}<\infty$.

${ }^{\dagger} C(\sigma(T))$ representa o espaço vetorial das funções contínuas em $\sigma(T)$, o espectro de $T$. 
Riesz-Markov (vide, por exemplo, o Teorema IV.14 de [RS1]) a existência de uma única medida $\mu_{\psi}$ no conjunto compacto $\sigma(T)$ (vide o Teorema A.1.9), tal que

$$
\langle f(T) \psi, \psi\rangle=\int_{\sigma(T)} f(\lambda) d \mu_{\psi}(\lambda) .
$$

Definição B.1.1 A medida $\mu_{\psi}$ é chamada de medida espectral associada ao vetor $\psi$.

A primeira aplicação da medida espectral $\mu_{\psi}$ é permitir a extensão da relação (B.1.1) de $C(\sigma(T)$ ) para $\mathcal{B}(\mathbb{R})$, o espaço das funções de Borel limitadas em $\mathbb{R}$. Seja $g \in \mathcal{B}$; é natural definirmos $g(T)$ de modo que $\langle g(T) \psi, \psi\rangle=\int_{\sigma(T)} g(\lambda) d \mu_{\psi}(\lambda)$ : a identidade de polarização nos permite obter $\langle g(T) \psi, \phi\rangle$ e então o lema de Riesz (Teorema II.4 de [RS1]) nos conduz a $g(T)$.

Definição B.1.2 Um vetor $\psi \in \mathcal{H}$ é chamado de vetor cíclico de $T$ se combinações lineares finitas dos elementos do conjunto $\left\{T^{n} \psi\right\}_{n=0}^{\infty}$ são densas em $\mathcal{H}$.

Nem todos os operadores possuem vetores cíclicos, mas caso isso ocorra, temos o

Lema B.1.3 (Lema 1 do Capítulo VII de [RS1]) Seja T um operador limitado autoadjunto com vetor cíclico $\psi$. Então, existe um operador unitário $U: \mathcal{H} \longrightarrow L^{2}\left(\sigma(T), d \mu_{\psi}\right)$ tal que

$$
\left(U T U^{-1} f\right)(\lambda)=\lambda f(\lambda) .
$$

A igualdade é no sentido dos elementos de $L^{2}\left(\sigma(T), d \mu_{\psi}\right)$.

Demonstração. Defina $U$ por $U \phi(f) \psi \equiv f$ onde $f$ é uma função contínua e $\phi$ : $C(\sigma(T)) \longrightarrow B(\mathcal{H}, \mathcal{H})$ é a aplicação definida no Teorema VII.1 de [RS1] tal que

(a) $\phi$ é um $*$-homomorfismo algébrico, isto é, $\phi(f g)=\phi(f) \phi(g), \phi(\lambda f)=\lambda \phi(f), \phi(1)=$ $I$ e $\phi(\bar{f})=\phi(f)^{*}$;

(b) $\phi$ é contínua, ou seja, $\|\phi(f)\|_{B(\mathcal{H}, \mathcal{H})} \leq C\|f\|_{\infty}$;

(c) Se $f(x)=x$, então $\phi(f)=T$;

(d) Se $T \psi=\lambda \psi$, então $\phi(f) \psi=f(\lambda) \psi$

(e) $\sigma[\phi(f)]=\{f(\lambda): \lambda \in \sigma(T)\}$ (teorema do mapeamento espectral).

Desse modo, $U$ é essencialmente o operador inverso de $\phi$. Mostremos que $U$ é bem definido. De fato,

$$
\|\phi(f) \psi\|^{2}=\langle\phi(\bar{f} f) \psi, \psi\rangle=\int|f(\lambda)|^{2} d \mu_{\psi},
$$

e caso $f=g$ quase certamente com respeito a $\mu_{\psi}$, então $\phi(f) \psi=\phi(g) \psi$. Assim, $U$ é bem definido em $\{\phi(f) \psi: f \in C(\sigma(T))\}$ e preserva a norma. Como $\psi$ é cíclico, $\overline{\{\phi(f) \psi: f \in C(\sigma(T))\}}=\mathcal{H}$, e assim $U$ se estende a um operador isométrico de $\mathcal{H}$ em 
$L^{2}\left(\sigma(T), d \mu_{\psi}\right)$. Como $C(\sigma(T))$ é denso em $L^{2}$, a imagem de $U$ é o espaço $L^{2}\left(\sigma(T), d \mu_{\psi}\right)$. Finalmente, se $f \in C(\sigma(T))$,

$$
\left(U T U^{-1} f\right)(\lambda)=[U T \phi(f)](\lambda)=[U \phi(x f)](\lambda)=\lambda f(\lambda) .
$$

Por continuidade, este resultado se estende de $f \in C(\sigma(T))$ a $f \in L^{2}$.

A fim de estender o Lema B.1.3 para um $T$ arbitrário, precisamos nos assegurar de que cada $T$ possui uma família de subespaços invariantes que geram $\mathcal{H}$, de modo que $T$ é cíclico em cada subespaço.

Lema B.1.4 (Lema 2 do Capítulo VII de [RS1]) Seja T um operador limitado autoadjunto em um espaço de Hilbert separável $\mathcal{H}$. Existe então uma decomposição $\mathcal{H}=$ $\oplus_{n=1}^{N} \mathcal{H}_{n}, N \geq 1$, tal que

(a) $T$ deixa cada $\mathcal{H}_{n}$ invariante, ou seja, $\psi \in \mathcal{H}_{n}$ resulta em $T \psi \in \mathcal{H}_{n}$.

(b) Existe, para cada $n$, um $\psi_{n} \in \mathcal{H}_{n}$ cíclico para a restrição $T_{n}$ de $T$ a $\mathcal{H}_{n}$, isto, é, $\mathcal{H}_{n}=\overline{\left\{f(T) \psi_{n}: f \in C(\sigma(T))\right\}}$.

Demonstração. O lema é decorrente de uma aplicação direta do Lema de Zorn.

Podemos combinar os Lemas B.1.3 e B.1.4 e obter o

Teorema B.1.5 (Teorema espectral - operador de multiplicação) Seja T um operador limitado auto-adjunto definido em um espaço de Hilbert separável $\mathcal{H}$. Então, existem medidas de Borel $\left\{\mu_{n}\right\}_{n=1}^{N}$ em $\sigma(T)$ e um operador unitário

$$
U: \mathcal{H} \longrightarrow \bigoplus_{n=1}^{N} L^{2}\left(\mathbb{R}, d \mu_{n}\right)
$$

de modo que

$$
\left(U T U^{-1} \psi\right)_{n}(\lambda)=\lambda \psi_{n}(\lambda),
$$

onde escrevemos um elemento $\psi \in \oplus_{n=1}^{N} L^{2}\left(\mathbb{R}, d \mu_{n}\right)$ como a $N$-tupla $\left(\psi_{1}(\lambda), \ldots, \psi_{N}(\lambda)\right)$. Esta realização de $T$ é chamada de representação espectral.

Demonstração. Basta usar o Lema B.1.4 a fim de obter a decomposição e o Lema B.1.3 em cada componente.

O Teorema B.1.5 nos diz que todo operador limitado auto-adjunto é um operador de multiplicação em um espaço mensurável apropriado. A mudança que ocorre quando trocamos de operador é na medida associada; explicitamente 
Corolário B.1.6 Seja T um operador limitado auto-adjunto definido em um espaço de Hilbert separável $\mathcal{H}$. Então, existe um espaço mensurável $(M, \mu)$, uma função limitada $F$ em $M$, e um operador unitário $U: \mathcal{H} \longrightarrow L^{2}(\mathbb{R}, d \mu)$, de modo que

$$
\left(U T U^{-1} f\right)(m)=F(m) f(m) .
$$

Demonstração. Escolha os vetores cíclicos $\psi_{n}$ tais que $\left\|\psi_{n}\right\|=2^{-n}$. Seja $M=\bigcup_{n=1}^{N} \mathbb{R}$, a união de $N$ cópias de $\mathbb{R}$. Defina $\mu$ impondo que sua restrição à $n$-ésima cópia de $\mathbb{R}$ seja $\mu_{n}$. Como $\mu(M)=\sum_{n=1}^{N} \mu_{n}(\mathbb{R})<\infty, \mu$ é finita.

Observação B.1.7 1. As medidas $d \mu_{n}$ também são chamadas de medidas espectrais; elas simplesmente são $d \mu_{\psi}$ para um $\psi$ apropriado.

2. Estas medidas não são unicamente determinadas; um estudo completo desse problema se encontra, por exemplo, na Seção 2 do Capítulo VII de [RS1].

Definição B.1.8 Se $\left\{\mu_{n}\right\}_{n=1}^{N}$ é uma família de medidas, o suporte de $\left\{\mu_{n}\right\}$ é o complemento do maior conjunto aberto $B$ com $\mu_{n}(B)=0$ para todo $n$, de modo que

$$
\Sigma\left(\left\{\mu_{n}\right\}\right)=\overline{\bigcup_{n=1}^{N} \Sigma\left(\mu_{n}\right)} .
$$

Proposição B.1.9 Seja $T$ um operador auto-adjunto e $\left\{\mu_{n}\right\}$ uma família de medidas espectrais. Então

$$
\sigma(T)=\Sigma\left(\left\{\mu_{n}\right\}_{n=1}^{N}\right)
$$

Demonstração. A demonstração da Proposição B.1.9 segue os mesmos passos da demonstração do Lema D.4.2.

Existe uma descrição simples de $\sigma(T)$ em termos de operadores de multiplicação mais gerais.

Definição B.1.10 Seja $F$ uma função a valores reais em um espaço mensurável $(M, \mu)$. Dizemos que $\lambda$ pertence à imagem essencial de $F$ se, e somente se,

$$
\mu(\{m: \lambda-\varepsilon<F(m)<\lambda+\varepsilon\})>0
$$

para todo $\varepsilon>0$.

Proposição B.1.11 Seja $F$ uma função a valores reais definida no espaço mensurável $(M, \mu)$. Seja $T_{F}$ o operador em $L^{2}(M, d \mu)$ dado por

$$
\left(T_{F} g\right)(m)=F(m) g(m) \text {. }
$$

Então $\sigma\left(T_{F}\right)$ é a imagem essencial de $F$. 
Demonstração. A Proposição B.1.11 é a generalização do Lema 2.3.6 para o espaço mensurável $(M, \mu)$; sua demonstração segue os mesmos passos da demonstração do lema mencionado.

Existe uma decomposição bastante natural da medida espectral (e de medidas de Borel-Stieltjes mais gerais) que envolve sua diferenciabilidade com respeito à medida de Lebesgue.

Primeiro, seja $P=\{x: \mu(\{x\}) \neq 0\}$, o conjunto dos pontos com massa positiva de $\mu$. Como $\mu$ é Borel $(\mu(C)<\infty$ para qualquer conjunto $C$ compacto), $P$ é um conjunto contável. Defina

$$
\mu_{\mathrm{pp}}(X)=\sum_{x \in P \cap X} \mu(\{x\})=\mu(P \cap X) .
$$

Então $\mu_{\mathrm{pp}}$ é uma medida de Borel e $\mu_{\mathrm{c}}=\mu-\mu_{\mathrm{pp}}$. A medida $\mu_{\mathrm{pp}}$ assim definida é tal que $\mu_{\mathrm{c}}(\{x\})=0$ para todo $x$ (isto é, não possui átomos) e $\mu_{\mathrm{pp}}$ possui apenas átomos, no sentido que $\mu_{\mathrm{pp}}(X)=\sum_{x \in X} \mu(x)$ para qualquer conjunto de Borel $X$.

Definição B.1.12 Uma medida de Borel $\mu$ é dita contínua se esta não possui átomos e puramente pontual se $\mu(X)=\sum_{x \in X} \mu(x)$ para qualquer conjunto de Borel $X$.

Assim, demonstramos o

Teorema B.1.13 (Teorema I.13 de [RS1]) Qualquer medida de Borel pode ser decomposta unicamente na soma $\mu=\mu_{p p}+\mu_{c}$, onde $\mu_{p p}$ é puramente pontual e $\mu_{c}$ é contínua.

Definição B.1.14 Dizemos que a medida de Borel $\mu$ é absolutamente contínua com respeito à medida de Lebesgue $\kappa$ se existe um função, f, localmente $L^{1 \dagger}$ tal que

$$
\int g(\lambda) d \mu(\lambda)=\int f(\lambda) g(\lambda) d \kappa(\lambda)
$$

para qualquer função de Borel $g \in L^{1}(\mathbb{R}, d \mu)$. A função $f$ é a derivada de Radon-Nikodym de $\mu$ com respeito a $\kappa$ (vide a Definição D.3.5).

Definição B.1.15 Dizemos que a medida $\mu$ é singular com respeito à medida de Lebesgue $\kappa$ se $\mu$ é suportado em um conjunto $S$ (isto é, $\mu(\mathbb{R} \backslash S)=0$ ) tal que $\kappa(S)=0$.

Temos o fundamental

Teorema B.1.16 (Teorema de decomposição de Lebesgue) Seja $\mu$ uma medida de Borel. Então $\mu=\mu_{a c}+\mu_{s}$ de uma maneira única, com a medida $\mu_{a c}$ absolutamente contínua e com a medida $\mu_{s}$ singular com com respeito à medida de Lebesgue $\kappa$.

${ }^{\dagger}$ Isto é, $\int_{a}^{b}|f(\lambda)| d \kappa(\lambda)<\infty$ para qualquer intervalo finito $(a, b)$. 
Os Teoremas B.1.13 e B.1.16 nos dizem que qualquer medida de Borel $\mu$ em $\mathbb{R}$ possui a decomposição canônica $\mu=\mu_{\mathrm{ac}}+\mu_{\mathrm{s}}=\mu_{\mathrm{ac}}+\mu_{\mathrm{pp}}+\mu_{\mathrm{sc}}$, onde $\mu_{\mathrm{sc}}$ é contínua (tal que $\mu_{\mathrm{sc}}(\{x\})=0$ para todo $x \in S$ ) e singular (chamada de singular-contínua) com respeito a $\kappa$, no sentido que $\mu_{\text {sc }}$ é suportada em um conjunto $S$, de medida de Lebesgue nula $(\kappa(S)=0)$.

Estas três medidas são mutuamente singulares (no sentido da Definição B.1.15), de modo que

$$
L^{2}(\mathbb{R}, d \mu)=L^{2}\left(\mathbb{R}, d \mu_{\mathrm{pp}}\right) \oplus L^{2}\left(\mathbb{R}, d \mu_{\mathrm{ac}}\right) \oplus L^{2}\left(\mathbb{R}, d \mu_{\mathrm{sc}}\right) .
$$

É possível mostrar (vide problema 18 do Capítulo VII de [RS1]) que qualquer $\psi \in$ $L^{2}(\mathbb{R}, d \mu)$ possui uma medida espectral absolutamente contínua $d \mu_{\psi}$ se, e somente se, $\psi \in$ $L^{2}\left(\mathbb{R}, d \mu_{\text {ac }}\right)$, o mesmo para as medidas puramente pontual e singular-contínua. Se $\left\{\mu_{n}\right\}_{n=1}^{N}$ é uma família de medidas espectrais, podemos somar, por exemplo, $\oplus_{n=1}^{N} L^{2}\left(\mathbb{R}, d \mu_{n ; \mathrm{ac}}\right)$, através da

Definição B.1.17 Seja T um operador limitado auto-adjunto, definido no espaço de Hilbert $\mathcal{H}$. Definimos $\mathcal{H}_{p p}=\left\{\psi: \mu_{\psi}\right.$ é puramente pontual $\}, \mathcal{H}_{a c}=\left\{\psi: \mu_{\psi}\right.$ é absolutamente contínua $\}$ e $\mathcal{H}_{s c}=\left\{\psi: \mu_{\psi}\right.$ é singular-contínua $\}$.

Assim, demonstramos, a partir da Definição B.1.17 e das considerações anteriores, o

Teorema B.1.18 (Teorema VII.4 de [RS1]) O espaço de Hilbert $\mathcal{H}$ pode ser decomposto na soma direta $\mathcal{H}=\mathcal{H}_{p p} \oplus \mathcal{H}_{a c} \oplus \mathcal{H}_{s c}$, de acordo com a Definição B.1.17. Cada um destes subespaços é invariante pela aplicação de $T$; a restrição $T \uparrow \mathcal{H}_{p p}$ possui um conjunto completo de autovetores, $T \uparrow \mathcal{H}_{a c}$ possui apenas medidas espectrais absolutamente contínuas e $T \uparrow \mathcal{H}_{\text {sc }}$ possui apenas medidas singular-contínuas.

Os resultados apresentados no Teorema B.1.18 nos levam a

Definição B.1.19 Sejam os conjuntos

$$
\begin{aligned}
& \sigma_{p p}(T)=\{x \in \sigma(T): \mu(\{x\}) \neq 0\} \\
& \sigma_{c}(T)=\{x: \mu(\{x\})=0\} \\
& \sigma_{a c}(T)=\left\{T \uparrow \mathcal{H}_{a c}\right\} \\
& \sigma_{s c}(T)=\left\{T \uparrow \mathcal{H}_{s c}\right\}
\end{aligned}
$$

Estes conjuntos são chamados, respectivamente, de espectros puramente pontual, contínuo, absolutamente contínuo e singular-contínuo.

Observação B.1.20 Diferentemente da Definição A.1.1, o espectro puramente pontual não é constituído apenas dos autovalores de T (o chamado espectro discreto); este pode conter pontos de acumulação dos próprios autovalores. Uma discussão mais precisa será apresentada mais adiante. 


\section{B.2 Projetores espectrais}

Comecemos pela

Definição B.2.1 Seja T um operador limitado auto-adjunto e $\Gamma$ um subconjunto de Borel de $\mathbb{R}$. Denominamos o operador $P_{\Gamma} \equiv \chi_{\Gamma}(T)$ de projetor espectral de $T$.

Como a Definição B.2.1 sugere, $P_{\Gamma}$ é um projetor espectral uma vez que $\chi_{\Gamma}^{2}=\chi_{\Gamma}=\bar{\chi}_{\Gamma}$ pontualmente. As propriedades da família de projetores $\left\{P_{\Gamma}: \Gamma\right.$ é um conjunto de Borel arbitrário\} são dadas pela

Proposição B.2.2 A família $\left\{P_{\Gamma}\right\}$ de projetores espectrais de um operador limitado e auto-adjunto $T$ possui as seguintes propriedades:

(a) Cada $P_{\Gamma}$ é um projetor ortogonal.

(b) $P_{\emptyset}=0, P_{(-a, a)}=I$ para algum a.

(c) $S e \Gamma=\bigcup_{n=1}^{\infty} \Gamma_{n}$, com $\Gamma_{n} \bigcap \Gamma_{m}=\emptyset$ para todos $n \neq m$, então

$$
P_{\Gamma}=\operatorname{s}_{N \rightarrow \infty} \sum_{n=1}^{N} P_{\Gamma_{n}}
$$

(s-lim é o limite na topologia forte de operadores $\left.{ }^{\dagger}\right)$.

(d) $P_{\Gamma_{1}} P_{\Gamma_{2}}=P_{\Gamma_{1} \cap \Gamma_{2}}$.

Observação B.2.3 Referenciamos a Seção VII.3 de [RS1] para a demonstração da Proposição B.2.2.

A condição (c) da Proposição B.2.2 lembra bastante a condição que define uma medida; de fato,

Definição B.2.4 Uma familia de projetores que satisfazem as condições (a)-(c) da Proposição B.2.2 é chamada de medida a valores projecionais (m.v.p).

Podemos integrar com respeito a uma m.v.p. De fato, se $P_{\Gamma}$ é uma m.v.p., então $\left\langle P_{\Gamma} \phi, \phi\right\rangle$ é uma medida comum para todo $\phi$. Pelo Lema de Riesz (Teorema II.2 de [RS1]), chegamos ao

Teorema B.2.5 Se $P_{\Gamma}$ é uma m.v.p. e f é uma função mensurável limitada, definida no suporte de $P_{\Gamma}$, então existe um único operador $B$, que denotamos por $\int f(\lambda) d P_{\lambda}$, tal que

$$
\langle B \phi, \phi\rangle=\int f(\lambda) d\left\langle P_{\lambda} \phi, \phi\right\rangle
$$

para todo $\phi \in \mathcal{H}$.

${ }^{\dagger}$ Nesta topologia, uma família de operadores $\left\{T_{\alpha}\right\}$ converge ao operador $T$ se, e somente se, $\| T_{\alpha} x-$ $T x \| \rightarrow 0$ para todo $x \in X$. 
Agora, dada uma m.v.p. limitada $P_{\Gamma}$, definimos $T=\int \lambda d P_{\lambda}$; é simples mostrar que $P_{\Gamma}$ é a m.v.p. associada a $T$. Resumindo, temos o

Teorema B.2.6 (Teorema espectral - forma projecional) Existe uma correspondência biunivoca entre operadores auto-adjuntos limitados $T$ e medidas a valores projecionais limitadas, $\left\{P_{\Gamma}\right\}$, dada por

$$
\begin{aligned}
& T \longmapsto\left\{P_{\Gamma}\right\}=\left\{\chi_{\Gamma}(T)\right\}, \\
& \left\{P_{\Gamma}\right\} \longmapsto T=\int \lambda d P_{\lambda} .
\end{aligned}
$$

Os projetores espectrais podem ser usados para investigar o espectro do operador $T$ :

Proposição B.2.7 O ponto $\lambda$ pertence ao espectro do operador auto-adjunto $T$ se, $e$ somente se, $P_{(\lambda-\varepsilon, \lambda+\varepsilon)} \neq 0$ para todo $\varepsilon>0$.

Demonstração. Devemos mostrar que $\lambda \in \rho(T)$ se, e somente se, $P_{(\lambda-\varepsilon, \lambda+\varepsilon)}=0$ para algum $\varepsilon>0$. Como $\sigma(T)=\mathbb{R} \backslash \rho(T)$, segue que $\lambda \in \sigma(T)$ se, e somente se, $P_{(\lambda-\varepsilon, \lambda+\varepsilon)} \neq 0$ para todo $\varepsilon>0$.

A fim de demonstrar a afirmação acima, usamos a relação

$$
\|(T-\lambda I) \phi\|^{2}=\int_{-\infty}^{\infty}(t-\lambda)^{2} d\left\langle P_{t} \phi, \phi\right\rangle,
$$

decorrente do Teorema espectral B.2.6, e válida para $\phi \in \mathcal{D}(T)$. Se $P_{(\lambda-\varepsilon, \lambda+\varepsilon)}=0$ para algum $\varepsilon>0$, segue da relação (B.2.1) que

$$
\|(T-\lambda I) \phi\|^{2} \geq \int_{(-\infty, \lambda-\varepsilon] \cup[\lambda+\varepsilon, \infty)}(t-\lambda)^{2} d\left\langle P_{t} \phi, \phi\right\rangle \geq \varepsilon^{2}\|\phi\|
$$

de onde concluímos (Teorema A.1.7) que $\lambda \in \rho(T)$.

Por outro lado, se $\lambda \in \rho(T)$, então, para algum $\varepsilon>0$ e para todo $\phi \in \mathcal{D}(T)$,

$$
\int_{-\infty}^{\infty}(t-\lambda)^{2} d\left\langle P_{t} \phi, \phi\right\rangle \geq \varepsilon^{2} \int_{-\infty}^{\infty} d\left\langle P_{t} \phi, \phi\right\rangle
$$

Assumamos a existência de um $0<\eta<\varepsilon$ e de um elemento $\psi$ tais que $P_{(\lambda+\eta, \lambda-\eta)} \psi \neq 0$. O uso da desigualdade (B.2.2) em $\phi=P_{(\lambda+\eta, \lambda-\eta)} \psi$ resulta em

$$
\int_{\lambda-\eta}^{\lambda+\eta}(t-\lambda)^{2} d\left\langle P_{t} \psi, \psi\right\rangle \geq \varepsilon^{2} \int_{\lambda-\eta}^{\lambda+\eta} d\left\langle P_{t} \psi, \psi\right\rangle
$$

desigualdade obviamente falsa. Isso conclui a demonstração da proposição.

A Proposição B.2.7 nos sugere distinguir dois tipos de espectro:

Definição B.2.8 Dizemos que $\lambda \in \sigma_{\text {ess }}(T)$, o espectro essencial de $T$, se, e somente se, $P_{(\lambda-\varepsilon, \lambda+\varepsilon)}(T)$ possui dimensão infinita para todo $\varepsilon>0$. Se $\lambda \in \sigma$, porém $P_{(\lambda-\varepsilon, \lambda+\varepsilon)}(T)$ possui dimensão finita para algum $\varepsilon>0$, dizemos que $\lambda \in \sigma_{d f}(T)$, o espectro discreto finito de $T$.

\footnotetext{
†O projetor $P$ ter dimensão infinita significa que a imagem de $P$ possui dimensão infinita.
} 
Teorema B.2.9 O espectro essencial de um operador auto-adjunto é sempre um conjunto fechado.

Demonstração. Seja $\lambda_{n} \rightarrow \lambda$, com cada $\lambda_{n} \in \sigma_{\text {ess }}(T)$. Como todo intervalo aberto $I$, centrado em $\lambda$, contém um intervalo que envolve algum $\lambda_{n}$, segue que $P_{I}(T)$ possui dimensão infinita, o que, pela Definição B.2.8, resulta em $\lambda \in \sigma_{\text {ess }}(T)$. Isso demonstra que $\sigma_{\text {ess }}(T)$ é fechado.

Os próximos três teoremas dão descrições alternativas de $\sigma_{\mathrm{df}}(T)$ e $\sigma_{\mathrm{ess}}(T)$.

Teorema B.2.10 (Teorema VII.10 de [RS1]) $\lambda \in \sigma_{d f}(T)$ se, e somente se, ambas afirmações são satisfeitas:

(a) $\lambda$ é um ponto isolado de $\sigma(T)$, ou seja, para algum $\varepsilon>0,(\lambda-\varepsilon, \lambda+\varepsilon) \cap \sigma(T)=\{\lambda\}$.

(b) $\lambda$ é um autovalor de multiplicidade finita, ou seja, o conjunto $\{\psi: T \psi=\lambda \psi\}$ possui dimensão finita.

Demonstração. O teorema segue diretamente da Definição B.2.8.

Teorema B.2.11 (Teorema VII.11 de [RS1]) $\lambda \in \sigma_{\text {ess }}(T)$ se, e somente se, uma ou mais das seguintes afirmações são satisfeitas:

(a) $\lambda \in \sigma_{c}(T) \equiv \sigma_{a c}(T) \cup \sigma_{s c}(T)$.

(b) $\lambda$ é um ponto de acumulação de $\sigma_{p p}(T)$.

(c) $\lambda$ é um autovalor de multiplicidade infinita, ou seja, o conjunto $\{\psi: T \psi=\lambda \psi\}$ possui dimensão infinita.

Demonstração. Como $\sigma_{\mathrm{df}}(T)$ e $\sigma_{\text {ess }}(T)$ são conjuntos disjuntos, para qualquer operador auto-adjunto $T$, obtemos o teorema diretamente do Teorema B.2.10 (observe que as afirmações deste teorema são complementares às afirmações do Teorema B.2.10).

Observação B.2.12 1. Repare que a Definição A.1.1 de espectro discreto consiste nos autovalores do operador $T$, sendo estes de multiplicidade finita ou não, enquanto que a parte puramente pontual do espectro essencial engloba tantos os autovalores de multiplicidade (geométrica) infinita quanto seus pontos de acumulação. De fato, podemos pensar no espectro puramente pontual (Definição B.1.19) como sendo a união disjunta dos conjuntos

$$
\sigma_{p p}(T)=\left(\sigma_{e s s}(T) \backslash \sigma_{c}(T)\right) \cup \sigma_{d f}(T) .
$$

Em nosso trabalho, interessamo-nos apenas no espectro essencial, de modo que por espectro puramente pontual nos referimos ao conjunto $\sigma_{\text {ess }}(T) \backslash \sigma_{c}(T)$; qualquer desvio de linguagem é prontamente notificado. 
2. É possivel mostrar que as Definições A.1.1 e B.1.19 de espectro contínuo, bem como sua parte no espectro essencial, coincidem. A saber, o que o item (a) do Teorema B.2.10 nos diz é que $\sigma_{c}(T) \subseteq \sigma_{\text {ess }}(T)$.

Teorema B.2.13 (Critério de Weyl) Seja T um operador limitado auto-adjunto. Então $\lambda \in \sigma(T)$ se, e somente se, existe uma seqüência $\left\{\psi_{n}\right\}_{n=1}^{\infty}$ de vetores unitários tais que $\lim _{n \rightarrow \infty}\left\|(T-\lambda I) \psi_{n}\right\|=0$. Por outro lado $\lambda \in \sigma_{\text {ess }}(T)$ se, e somente se, os vetores acima podem ser escolhidos como sendo ortogonais.

Finalmente, demonstramos o chamado princípio de invariância de Weyl, fundamental nos resultados apresentados nesse trabalho.

Proposição B.2.14 (Princípio de invariância de Weyl) Sejam A e B dois operadores limitados auto-adjuntos tais que $A-B$ é um operador de posto finito (e portanto compacto). Então $\sigma_{\text {ess }}(A)=\sigma_{\text {ess }}(B)$.

Demonstração. Escreva $A=B+C$, com $C$ compacto e de posto finito. Defina $F(z)=C(A-z I)^{-1}$ para $z \in \mathbb{C} \backslash \sigma_{\text {ess }}(A)$. Então $F(z)$ é uma função a valores vetoriais analítica que é sempre fechada, já que $C$ é compacto e de posto finito por hipótese. Sendo $A$ um operador limitado, segue que

$$
\lim _{z \rightarrow \infty}\|F(z)\| \leq\|C\|\left\|(A-z I)^{-1}\right\|=0 .
$$

Em particular, $(I-F(z))^{-1}$ existe se $|z|$ é grande. Pelo Teorema meromórfico de Fredholm (Teorema XIII.13 de [RS4]) e pelo Lema 1 da Seção XIII.4 de [RS4], (I $F(z))^{-1}$ existe para $z \in \mathbb{C} \backslash\left(\sigma_{\text {ess }}(A) \cup D\right), D$ um subconjunto discreto (ou seja, constituído unicamente por pontos) de $z \in \mathbb{C} \backslash \sigma_{\text {ess }}(A)$; mais ainda, $(I-F(z))^{-1}$ é meromórfica em $z \in \mathbb{C} \backslash \sigma_{\text {ess }}(A)$, com resíduos de posto finito nos pontos de $D$. Se $z \notin \sigma_{\text {ess }}(A)$, então $B-z I=\left(I-C(A-z I)^{-1}\right)(A-z I)$, de modo que se $(I-F(z))^{-1}$ é inversível, de modo que $z \notin \sigma(B)$ e $(B-z I)^{-1}=(A-z I)^{-1}(I-F(z))^{-1}$. Assim, $\sigma(B) \subset D \cap \sigma_{\text {ess }}(A)$.

Além do mais, $(B-z I)^{-1}$ possui resíduos de posto finito nos pontos de $D$. Logo, os pontos de $D$ pertencem a $\sigma_{\mathrm{df}}(B)$, de modo que $\sigma_{\text {ess }}(B) \subset \sigma_{\text {ess }}(A)$. Da mesma forma, demonstramos que $\sigma_{\text {ess }}(A) \subset \sigma_{\text {ess }}(B)$, o que nos permite concluir a demonstração da proposição. 


\section{Apêndice C}

\section{Funções Herglotz}

As funções Herglotz (ou Nevanlinna, ou ainda Pick) são funções $f(z)=U(z)+i V(z)$, analíticas no semi-plano complexo superior, com parte imaginária positiva; desse modo, se $z=\xi+i \eta$, então $V(z) \geq 0$ caso $\eta>0$. Elas evidentemente formam um cone convexo: por, exemplo, se $a$ e $b$ são números positivos e $f_{1}(z), f_{2}(z)$ duas funções Herglotz, então a função $a f_{1}(z)+b f_{2}(z)$ também o é. Podemos destacar algumas outras propriedades: a função $\left(f_{2} \circ f_{1}\right)(z)=f_{2}\left(f_{1}(z)\right)$ é analítica para $\eta>0$ e possui parte imaginária positiva nessa região; como a função $-1 / z$ é Herglotz, segue que $-1 / f(z)$ é Herglotz caso $f(z)$ o seja.

Um exemplo bastante sofisticado de função Herglotz é a chamada função de Green associada ao operador $H: f(z)=\left\langle(H-z I)^{-1} u, u\right\rangle$, onde $u$ é um vetor arbitrário pertencente ao espaço de Hilbert onde se define $H$ (vide a demonstração do Lema D.2.1 para uma discussão detalhada).

As funções Herglotz admitem a seguinte representação canônica

Teorema C.1 (Teorema I do Capítulo 2 de [Do1]) Uma função Herglotz $f(z)$ possui uma representação canônica única da forma

$$
f(z)=a z+b+\int\left[\frac{1}{\lambda-z}-\frac{\lambda}{\lambda^{2}+1}\right] d \mu(\lambda),
$$

onde $a \geq 0, b \in \mathbb{R}$ e $d \mu(\lambda)$ é uma medida de Borel positiva em $\mathbb{R}$ tal que a integral $\int\left(\lambda^{2}+1\right)^{-1} d \mu(\lambda)$ é finita. Por outro lado, toda função dessa forma é Herglotz.

Existe um resultado equivalente para funções positivas e harmônicas em $\mathbb{C}_{+}$.

Teorema C.2 (Teorema II do Capítulo 2 de [Do1]) Toda função positiva e harmônica em $\mathbb{C}_{+}$admite uma única representação canônica da forma

$$
V(\xi, \eta)=a \eta+\int \frac{\eta d \mu(\lambda)}{(\lambda-\xi)^{2}+\eta^{2}},
$$

onde $a \geq 0$ e d $\mu(\lambda)$ é uma medida de Borel positiva em $\mathbb{R}$ tal que a integral $\int\left(\lambda^{2}+\right.$ $1)^{-1} d \mu(\lambda)$ é finita. Por outro lado, toda função dessa forma é positiva e harmônica em $\mathbb{C}_{+}$. 
Antes de aprersentarmos as demonstrações, é interessante destacar que cada um dos teoremas pode ser deduzido do outro. A fim de demonstrarmos o Teorema C.2, por exemplo, podemos supor $V(\xi, \eta)$ dado, harmônico e positivo em $\mathbb{C}_{+}$. Tal função possui um conjugado harmônico (através das equações de Cauchy-Riemann) $U(\xi, \eta)$, determinado a menos de uma constante aditiva real. Assim, a função analítica $f(z)=U(z)+i V(z)$ é Herglotz e admite a representação (C.1), onde apenas a constante $b$ é indeterminada. Se tomarmos a parte imaginária de $f$ na representação (C.1), obtemos $V(z)$ na representação (C.2) sem ambigüidades, já que $b$ não faz parte da expressão.

Por outro lado, o Teorema C.1 é obtido a partir do Teorema C.2 escrevendo $V(z)$, a parte imaginária de $f(z)$, na forma (C.2) e notando que a função definida por (C.1), com $a$ e $\mu$ como em (C.2) e $b \equiv \Re f(i)$, é uma função analítica em $\mathbb{C}_{+}$, com o valor correto em $z=i$. Logo, (C.1) é uma representação da função Herglotz $f$. A representação é única, uma vez que $V(z)$, graças ao Teorema C.2, determina completamente $a$ e $\mu$.

Existem dois teoremas, análogos aos anteriores, para funções definidas no disco unitário.

Teorema C.3 (Teorema III do Capítulo 2 de [Do1]) Uma função $h(\zeta)=u(\zeta)+$ $i v(\zeta)$, analítica no disco $|\zeta|<1$ e com parte real positiva, admite uma única representação canônica da forma

$$
h(\zeta)=\int_{0}^{2 \pi} \frac{e^{i \theta}+\zeta}{e^{i \theta}-\zeta} d \omega(\theta)+i v(0),
$$

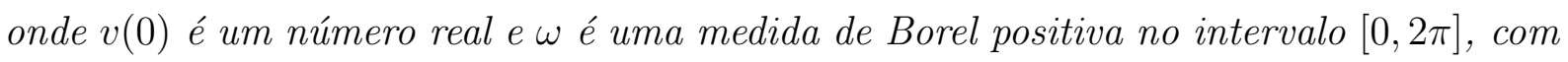
massa total finita. Por outro lado, toda função dessa forma é analítica no disco $\zeta<1$ e possui parte real positiva nele.

Teorema C.4 (Teorema IV do Capítulo 2 de [Do1]) Uma função $u(\zeta)$, positiva e harmônica em $|\zeta|<1$, admite uma única representação canônica da forma

$$
u(\zeta)=u\left(r e^{i \theta}\right)=\int_{0}^{2 \pi} \frac{1-r^{2}}{1+r^{2}-2 r \cos (\theta-\phi)} d \omega(\phi)
$$

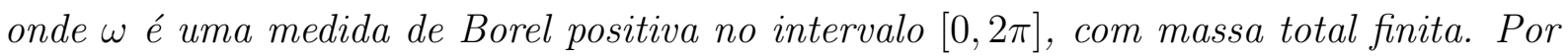
outro lado, toda função dessa forma é positiva e harmônica no círculo.

A equivalência dos Teoremas C.3 e C.4 é novamente obtida a partir do fato do harmônico conjugado ser determinado a menos de uma constante aditiva real. Vale notar que, para $\zeta=r e^{i \theta}$

$$
\frac{e^{i \theta}+\zeta}{e^{i \theta}-\zeta}=\frac{1-r^{2}-2 i r \sin (\theta-\phi)}{1+r^{2}-2 i r \cos (\theta-\phi)} .
$$

Mostramos a seguir a equivalência dos teoremas usando a função

$$
z(\zeta)=\frac{1}{i} \frac{\zeta+1}{\zeta-1}
$$


e sua inversa

$$
\zeta(z)=\frac{z-i}{z+i}
$$

um par de transformações lineares fracionárias (transformações de Möbius). A função $z(\zeta)$ mapeia o disco unitário sobrejetivamente no semi-plano superior, enquanto $\zeta(z)$ mapeia o semi-plano sobrejetivamente no disco unitário.

Se $h(\zeta)=u(\zeta)+i v(\zeta)$ é analítica no disco $|\zeta|<1$ com parte real positiva, a função $f(z)=i h(\zeta(z))$ é Herglotz; por outro lado, se $f(z)$ é Herglotz, a função $h(\zeta)=-f(z(\zeta))$ é analítica no disco, com parte real positiva. Assim, as duas classes de funções estão em correspondência biunívoca. Podemos, portanto, obter a representação canônica de $f(z)$ a partir da representação canônica da $h(\zeta)$ correspondente da seguinte forma.

Suponha $h(\zeta)$ dada por (C.3). Se a medida $d \omega$ possui uma massa pontual (ou átomo) $a>0$ em $\theta=0$, separamo-la, reescrevendo (C.3) como

$$
h(\zeta)=\frac{1+\zeta}{1-\zeta} a-i b+\int \frac{e^{i \theta}+\zeta}{e^{i \theta}-\zeta} d \omega^{\prime}(\theta)
$$

onde $d \omega^{\prime}$ é a medida obtida de $d \omega$ omitindo a massa pontual $a$ em $\theta=0$. Compomos $i h(\zeta(z))$; as duas primeiras parcelas do membro direito de (C.5) se reduzem a $a z+b$. Ao efetuarmos uma mudança de variável na integral, devemos apenas compor a transformação $\zeta(z) \operatorname{com}\left(e^{i \theta}+\zeta\right) /\left(e^{i \theta}-\zeta\right)$ e multiplicá-la por $i$, de modo que

$$
i \frac{e^{i \theta}+\frac{z-i}{z+i}}{e^{i \theta}-\frac{z-i}{z+i}}=i \frac{z\left(e^{i \theta}+1\right)+i\left(e^{i \theta}-1\right)}{z\left(e^{i \theta}-1\right)+i\left(e^{i \theta}+1\right)}=\frac{z \cos \theta / 2-\sin \theta / 2}{z \sin \theta / 2+\cos \theta / 2} ;
$$

em seguida, efetuamos a mudança de variável $\lambda=-\cot \theta / 2$, que mapeia o círculo sobrejetivamente no eixo real, e o ponto eliminado $z=1$ no infinito. Desse modo, $d \omega^{\prime}$ se torna uma medida de massa total finita no eixo real, de modo que

$$
f(z)=a z+b+\int \frac{\lambda z+1}{\lambda-z} d \nu(\lambda)
$$

onde $\int d \nu(\lambda)<\infty$. Escrevendo, finalmente, $d \mu(\lambda)=\left(\lambda^{2}+1\right) d \nu(\lambda)$, obtemos (C.1).

Todo o argumento anterior pode ser revertido: se uma função Herglotz $f(z)$ possui a representação (C.1), então a função $h(\zeta)=-i f(z(\zeta))$ é da forma (C.3). Concluímos, assim, que todos os teoremas apresentados são equivalentes, de modo que basta demonstrar um deles: provemos o Teorema C.4.

Demonstração do Teorema C.4. Para tanto, suponha em princípio que a função $u(\zeta)=$ $u\left(r e^{i \theta}\right)$ é positiva e harmônica em um disco de raio maior que 1; esta é, portanto, limitada e contínua em $|\zeta|=1$. Podemos determinar o conjugado harmônico $v(\zeta)$ sob a condição de que este se anula em $\zeta=0$; agora, $h(\zeta)=u(\zeta)+i v(\zeta)$ é uma função analítica em um disco de raio maior que 1 , e assim, pode ser representada pela série de potências $\sum a_{n} \zeta^{n}$, que converge absolutamente e uniformemente no círculo $|z|=1$. A parte real $u(\zeta)$ é então 
expressa pela série

$$
u(\zeta)=\frac{h(\zeta)+\overline{h(\zeta)}}{2}=\frac{1}{2} \sum_{n=1}^{\infty}\left[a_{n} z^{n}+\bar{a}_{n} \bar{z}^{n}\right]+a_{0}
$$

que também converge absolutamente e uniformemente para $|z| \leq 1$. Logo, nossa função admite a representação

$$
u(r, \theta)=\sum_{n=-\infty}^{\infty} A_{n} r^{|n|} e^{i n \theta},
$$

sendo simples notar que os coeficientes $A_{n}$ são os coeficientes de Fourier da função $u(1, \theta)$, de tal forma que

$$
u(r, \theta)=\frac{1}{2 \pi} \sum_{n=-\infty}^{\infty} r^{|n|} e^{i n \theta} \int_{0}^{2 \pi} u\left(e^{i \phi}\right) e^{-i n \phi} d \phi ;
$$

podemos, caso $r<1$, trocar a ordem entre integral e soma e obter

$$
\frac{1}{2 \pi} \int_{0}^{2 \pi} \sum_{n=-\infty}^{\infty} r^{|n|} e^{i n(\theta-\phi)} u\left(e^{i \phi}\right) d \phi,
$$

de modo que

$$
u(\zeta)=u(r, \theta)=\frac{1}{2 \pi} \int_{0}^{2 \pi} \frac{1-r^{2}}{1+r^{2}-2 r \cos (\theta-\phi)} u\left(e^{i \phi}\right) d \phi
$$

Esta é justamente a representação (C.4) para $u(\zeta)$, onde $d \omega(\phi)=(1 / 2 \pi) u\left(e^{i \theta}\right) d \phi, d \phi$ a medida de Lebesgue no intervalo $[0,2 \pi]$. A massa total dessa medida é dada pelo valor de $u(\zeta)$ na origem:

$$
u(0)=\frac{1}{2 \pi} \int_{0}^{2 \pi} u\left(e^{i \theta}\right) d \phi .
$$

O caso geral é conseqüência imediata do seguinte: se $u(\zeta)$ é uma função positiva e harmônica em $|z|<1$, a função $u_{\varepsilon}(\zeta)=u(\zeta /(1+\varepsilon)$ ) é positiva e harmônica no disco $|z|<1+\varepsilon$ e admite a representação (C.4), com uma medida positiva $d \omega_{\varepsilon}(\theta)$ de massa total $u_{\varepsilon}(0)=u(0)$. À medida que $\varepsilon \searrow 0$, as funções $u_{\varepsilon}(\zeta)$ convergem para $u(\zeta)$ uniformemente em subconjuntos compactos de $|\zeta|<1$; ao mesmo tempo, o sistema de medidas positivas $d \omega_{\varepsilon}$ satisfaz a hipótese do Teorema de seleção de Helly (vide p. 233 de [CL]), já que existe um limite superior uniforme para suas massas. Existe, portanto, uma seqüência $\varepsilon_{n}$ convergente a zero tal que a seqüência correspondente de medidas $d \omega_{n}$ converge, no sentido, fraco ${ }^{\dagger}$ para uma medida positiva $d \omega$, de tal forma que

$$
u(\zeta)=\lim _{\varepsilon_{n} \downarrow 0} u_{\varepsilon}(\zeta)=\int \frac{1-r^{2}}{1+r^{2}-2 r \cos (\theta-\phi)} d \omega(\phi) .
$$

\footnotetext{
${ }^{\dagger}$ Mais precisamente, $\lim _{n \rightarrow \infty} \int_{0}^{2 \pi} g(\phi) d \omega_{n}(\phi)=\int_{0}^{2 \pi} g(\phi) d \omega(\phi)$ para qualquer função $g$ contínua no intervalo $[0,2 \pi]$.
} 
Resta ainda demonstrarmos a unicidade das representações canônicas dadas por esses teoremas. Será suficiente mostrá-lo para a representação (C.1); para tanto, devemos verificar que os elementos $a, b$ e $d \mu$ são univocamente determinados pela função Herglotz $f(z)$.

É bastante claro que $b=\Re f(i)$, e que portanto $f$ determina $b$. Para mostrar que $a$ é unívocamente determinado, usamos a representação (C.6), a fim de estimar a razão $f(i \eta) / i \eta$ no limite assintótico $\eta \rightarrow \infty$. A razão em questão é igual a

$$
a+\frac{b}{i \eta}+\int \frac{\lambda^{2}+1+i \lambda(\eta+1 / \eta)}{\lambda^{2}+\eta^{2}} d \nu(\lambda)
$$

É bastante claro que o integrando converge pontualmente a 0 no limite $\eta \rightarrow \infty$, sendo suas partes real e imaginária uniformemente limitadas por 1 para todo $\eta>1$. Assim, pelo Teorema da convergência dominada de Lebesgue (Teorema 1.34 de [Ru]), o limite da integral é zero, o que nos permite concluir que

$$
a=\lim _{\eta \rightarrow \infty} \frac{f(i \eta)}{i \eta}
$$

o mesmo argumento mostra que $a=\lim _{\eta \rightarrow \infty} V(i \eta) / \eta$.

Resta mostrarmos que a medida $d \mu$ é determinada unicamente. Para tanto, necessitamos do

Lema C.5 (Lema 1 do Capítulo 2 de [Do1]) Para todo intervalo finito $\lambda_{1}<\lambda<\lambda_{2}$, segue que

$$
\mu\left(\left(\lambda_{1}, \lambda_{2}\right)\right)+\frac{1}{2}\left[\mu\left(\left\{\lambda_{1}\right\}\right)+\mu\left(\left\{\lambda_{2}\right\}\right)\right]=\lim _{\eta \downarrow 0} \frac{1}{\pi} \int_{\lambda_{1}}^{\lambda_{2}} \Im f(\lambda+i \eta) d \lambda .
$$

Demonstração. Obtemos, integrando $V(\lambda+i \eta)$ no intervalo $\lambda_{1}<\lambda<\lambda_{2}$,

$$
\int_{\lambda_{1}}^{\lambda_{2}} V(\lambda+i \eta)=\int_{-\infty}^{\infty} \chi_{\lambda_{1}, \lambda_{2}}(\eta, \lambda) d \mu(\lambda)
$$

onde

$$
\chi_{\lambda_{1}, \lambda_{2}}(\eta, \lambda)=\int_{\frac{\lambda_{1}-\lambda}{\eta}}^{\frac{\lambda_{2}-\lambda}{\eta}} \frac{d t}{1+t^{2}}=\arctan \left(\frac{\lambda_{2}-\lambda}{\eta}\right)-\arctan \left(\frac{\lambda_{1}-\lambda}{\eta}\right) .
$$

Claramente

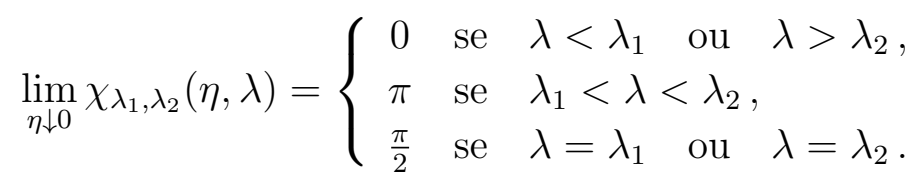

Desse modo, podemos reescrever (C.8) como

$$
\int_{\lambda_{1}}^{\lambda_{2}} V(\lambda+i \eta)=\int_{\lambda_{1}-1}^{\lambda_{2}+1} \chi_{\lambda_{1}, \lambda_{2}}(\eta, \lambda) d \mu(\lambda)+I(\eta)
$$


com

$$
\begin{aligned}
I(\eta) & =\int_{\lambda_{1}}^{\lambda_{2}} d \lambda\left(\int_{\lambda \geq \lambda_{2}+1} \frac{\eta d \mu(\lambda)}{(s-\lambda)^{2}+\eta^{2}}+\int_{\lambda \leq \lambda_{1}-1} \frac{\eta d \mu(\lambda)}{(s-\lambda)^{2}+\eta^{2}}\right) \\
& \leq\left(\lambda_{2}-\lambda_{1}\right)\left(\int_{\lambda \geq \lambda_{2}+1} \frac{\eta d \mu(\lambda)}{\left(s-\lambda_{2}\right)^{2}+\eta^{2}}+\int_{\lambda \leq \lambda_{1}-1} \frac{\eta d \mu(\lambda)}{\left(s-\lambda_{1}\right)^{2}+\eta^{2}}\right) .
\end{aligned}
$$

Como

$$
\frac{\eta}{\left(s-\lambda_{2}\right)^{2}+\eta^{2}} \leq \frac{1}{\left(s-\lambda_{2}\right)^{2}+1} \in L^{1}(d \mu)
$$

para todo $\lambda-\lambda_{2}>1>\eta\left(L^{1}(d \mu)\right.$ é o espaço das funções $d \mu$-integráveis, no sentido da integral de Borel-Stieltjes) e

$$
\lim _{\eta \downarrow 0} \frac{\eta}{\left(s-\lambda_{2}\right)^{2}+\eta^{2}}=0
$$

caso $\lambda \geq \lambda_{2}+1$, segue do Teorema da convergência dominada que

$$
\int_{\lambda \geq \lambda_{2}+1} \frac{\eta d \mu(\lambda)}{\left(s-\lambda_{2}\right)^{2}+\eta^{2}} \rightarrow 0
$$

à medida que $\eta \downarrow 0$. O mesmo argumento nos leva a

$$
\lim _{\eta \downarrow 0} \int_{\lambda \leq \lambda_{1}-1} \frac{\eta d \mu(\lambda)}{\left(s-\lambda_{1}\right)^{2}+\eta^{2}}=0
$$

de onde concluímos que $\lim _{\eta \downarrow 0} I(\eta)=0$.

Por outro lado, como $0 \leq \chi_{\lambda_{1}, \lambda_{2}}(\eta, \lambda) \leq \pi$ e $\pi \int_{\lambda_{1}-1}^{\lambda_{2}+1} d \mu(\lambda)<\infty$, segue da aplicação do Teorema da convergência dominada a (C.9) e dos resultados anteriores que

$$
\lim _{\eta \downarrow 0} \int_{\lambda_{1}}^{\lambda_{2}} V(\lambda+i \eta) d \lambda=\pi \mu((a, b))+\frac{\pi}{2}[\mu(\{a\})+\mu(\{b\})],
$$

exatamente (C.7), como queríamos demonstrar.

Com isso, encerramos a demonstração do Teorema C.4. 


\section{Apêndice D}

\section{Função $m$ de Weyl-Titchmarsh e seu papel na teoria espectral}

O objetivo desse apêndice é o de definir a função $m$ de Weyl-Titchmarsh e apresentar sua relação com a teoria espectral, especialmente com a medida espectral. Analisamos apenas o problema discreto; para uma discussão detalhada do problema contínuo vide [P1].

\section{D.1 Definições de matriz de Jacobi e função $m$}

Seja $T$ um operador auto-adjunto (Definição A.2) atuando em um espaço de Hilbert separável $\mathcal{H}$, e seja $f \in \bigcap_{k=1}^{\infty} D\left(T^{k}\right)$, onde $D\left(T^{k}\right)$ é o domínio da $k$-ésima potência de $T$ (tais vetores $f$ são densos em $\mathcal{H}$ ). Assumindo que $f$ não é uma combinação linear de um número finito de autovetores de $T$, segue que a seqüência $\left\{f, T f, T^{2} f, \ldots\right\}$ é linearmente independente. Podemos assim aplicar o processo de Gram-Schmidt e obter uma seqüência de vetores ortonormais $\left\{f_{n}\right\}_{n=0}^{\infty}$, onde cada $f_{n}$ é da forma $f_{n}=P_{n}(T) f, P_{n}$ um polinômio de ordem $n$, escolhido real ( $T$ é auto-adjunto, por definição). Estes são determinados a menos de um sinal (vide o Capítulo 2 de [D]), e seus coeficientes podem ser expressos em termos dos vários elementos de matriz $\left\langle T^{k} f, f\right\rangle$.

Definimos uma matriz infinita $L$, cujos componentes $L_{i j}, i, j=0,1,2, \ldots$, são expressos como

$$
L_{i j}=\left\langle T f_{i}, f_{j}\right\rangle=L_{j i}
$$

É simples notar que a matriz $L$ é tridiagonal no sentido que $L_{i j}=0$ se $|i-j|>1$ e $L_{i j} \neq 0$ caso contrário. A saber, podemos representar $L$ como

$$
L=\left(\begin{array}{ccccc}
b_{0} & a_{0} & 0 & 0 & \cdots \\
a_{0} & b_{1} & a_{1} & 0 & \cdots \\
0 & a_{1} & b_{2} & a_{2} & \cdots \\
0 & 0 & a_{2} & b_{3} & \cdots \\
\vdots & \vdots & \vdots & \vdots & \ddots
\end{array}\right)
$$


onde os $b(n)$ e os $a(n) \neq 0$ são números reais.

Assim, dado um operador auto-adjunto $T$ e um vetor genérico $f \in \bigcap_{k=1}^{\infty} D\left(T^{k}\right)$, somos imediatamente levados a definir uma matriz infinita, hermiteana e tridiagonal $L$, também conhecida como matriz de Jacobi. De fato, o operador $-\Delta_{P}$, proposto por Marchetti et.al. (definido por (1.4)) representa um caso particular de $T$, cuja matriz de Jacobi $J_{P}$ (expressa por (1.13)) corresponde a $L$ desde que $a_{n}=p_{n}$ e $b_{n}=0$. Se $T$ é limitado (como $-\Delta_{P}$, por exemplo), a matriz $L\left(J_{P}\right)$ pode ser tratada como representando a ação de $T$ no subespaço linear $\mathcal{H}_{f}$ gerado por $\left\{f, T f, T^{2} f, \ldots\right\}$. Se $T$ não é limitado, algumas complicações podem aparecer.

A condição para que a matriz $L$ defina unicamente a restrição de $T$ ao subespaço $\mathcal{H}_{f}$ é que a equação de Schrödinger associada seja ponto-limite em vez de círculo-limite (veremos mais adiante as definições desses conceitos). Neste caso, a ação do operador auto-adjunto $T$ em $\mathcal{H}_{f}$ é expressa por $T x=y$, em que $x=\sum_{i=0}^{\infty} x_{i} f_{i}, y=\sum_{i=0}^{\infty} y_{i} f_{i}$, e $y_{i}=\sum_{k} L_{i k} x_{k}$ (com $x \in D(T)$ e desde que $y \in \mathcal{H}_{f}$ ), de modo que podemos pensar na aplicação de $T$ a $\mathcal{H}_{f}$ como sendo equivalente a ação da matriz de Jacobi $L$ no espaço $l^{2}\left(\mathbb{Z}_{+}, \mathbb{C}\right)$

Observação D.1.1 De fato, $\mathcal{H}_{f} \cong l^{2}\left(\mathbb{Z}_{+}, \mathbb{C}\right)$, ou seja, os operadores $T$, definido no espaço de Hilbert separável $\mathcal{H}_{f}$, e L, a matriz de Jacobi definida em $l^{2}\left(\mathbb{Z}_{+}, \mathbb{C}\right)$, são unitariamente equivalentes $\left(L=U^{-1} T U\right.$, onde $\left.U: l^{2}\left(\mathbb{Z}_{+}, \mathbb{C}\right) \longrightarrow \mathcal{H}_{f}\right)$.

Considere a equação de Schrödinger (por vezes denotada equação de diferença finita ou ainda relação de recorrência)

$$
a_{n} u_{n+1}+a_{n-1} u_{n-1}+\left(b_{n}-z\right) u_{n}=0
$$

$n=1,2,3, \ldots, z \in \mathbb{C}$, associada à matriz de Jacobi $L$. Dessa forma, $u(z)$, o vetor coluna que representa a seqüência $\left\{u_{n}\right\}_{n=0}^{\infty}$ (solução de (D.1.2)), é uma solução formal da equação matricial $(L-z) u(z)=0$, com exceção da primeira componente, que satisfaz

$$
a_{-1} u_{-1}+\left(b_{0}-z\right) u_{0}+a_{0} u_{1}=0,
$$

onde, por conveniência, adotamos $a_{-1} \equiv 1$; uma outra escolha qualquer representaria uma mudança na condição de contorno definida abaixo, podendo ser imediatamente absorvida (vale notar que a escolha $a_{-1} \equiv 1$ equivale a escolha $p_{-1} \equiv 1$ no modelo de Marchetti et. al.). Dizemos que a matriz $L$ satisfaz uma condição de contorno $\alpha$ em $n=-1$ se $u_{-1}$ é expressa por

$$
u_{-1} \cos \alpha-u_{0} \sin \alpha=0 \text {. }
$$

Veremos mais adiante que a condição de contorno (D.1.4) desempenha um papel importante na determinação da matriz resolvente e na caracterização da parte singular do espectro. 
Para um z fixo, o conjunto das soluções da equação de Schrödinger é um espaço vetorial de dimensão 2 (trata-se de uma equação de diferença finita de segunda ordem), cuja base conveniente é definida pelas soluções $u^{D}(z)$ e $u^{N}(z)$, sujeitas às condições iniciais

$$
\begin{array}{ll}
u_{-1}^{D}=0, & u_{0}^{D}=1 \\
u_{-1}^{N}=1, & u_{0}^{N}=0
\end{array}
$$

(condições de Dirichlet e Neumann, respectivamente).

De uma forma mais geral, podemos adotar a base

$$
\begin{array}{ll}
u_{-1}^{\alpha}=\sin \alpha, & u_{0}^{\alpha}=\cos \alpha \\
u_{-1}^{\alpha^{*}}=\cos \alpha, & u_{0}^{\alpha^{*}}=-\sin \alpha,
\end{array}
$$

em que a solução $u^{\alpha}(z)$ satisfaz a condição de contorno (D.1.4) e $u^{\alpha^{*}}(z)$ é simplesmente a solução com condições iniciais ortogonais $\left(\alpha^{*} \equiv \alpha+\pi / 2^{\dagger}\right)$.

Para $\Im z \neq 0$, duas possibilidades podem ocorrer: temos ou o caso círculo-limite, no qual $u^{D}(z), u^{N}(z) \in l^{2}\left(\mathbb{Z}_{+}, \mathbb{C}\right)$ (e portanto toda solução da equação de Schrödinger (D.1.2)), ou o caso ponto-limite, no qual existe exatamente uma solução linearmente independente de (D.1.2) que pertence a $l^{2}\left(\mathbb{Z}_{+}, \mathbb{C}\right)$.

Proposição D.1.2 Seja L a matriz de Jacobi (D.1.1) associada ao operador auto-adjunto T. Se $\sum_{n=0}^{\infty}\left|a_{n}\right|^{-1}=\infty$, então L é ponto-limite.

Observação D.1.3 A identidade $\sum_{n=0}^{\infty}\left|a_{n}\right|^{-1}=\infty$ representa uma condição suficiente à existência do caso ponto-limite, ou seja, que L defina unicamente um operador autoadjunto $\mathrm{T}$ em $\mathcal{H}_{f}$.

Demonstração. Necessitamos, para a demonstração, da chamada fórmula de Green, de fundamental importância no decorrer do trabalho:

$$
\sum_{n=-1}^{N}\left(\bar{g}_{n}(L f)_{n}-\overline{(L g)}_{n} f_{n}\right)=W[f, g](N)-W[f, g](-1)
$$

onde definimos o Wronskiano $W[f, g](n)$ de duas funções $f, g: \mathbb{Z}_{+} \mapsto \mathbb{C}$ a partir da relação

$$
W[f, g](n):=a_{n}\left(f_{n} \bar{g}_{n+1}-f_{n+1} \bar{g}_{n}\right) \text {. }
$$

Em particular, se $f$ e $g$ são duas soluções da equação de Schrödinger (D.1.2), então a fórmula de Green aplicada a $f$ e $\bar{g}$ mostra que $W[f, g](n)$ é uma constante $\left(\sum_{n}\left[\bar{g}_{n}(L f)_{n}-\right.\right.$ $\left.\left.\overline{(L g)_{n}} f_{n}\right]=0\right)$ independente de $n$. Dessa forma, como $W\left[u^{D}, u^{N}\right](-1)=-W\left[u^{N}, u^{D}\right](-1)$ $=-a_{-1}=-1$, segue que $W\left[u^{D}, u^{N}\right](n)=-W\left[u^{N}, u^{D}\right](n)=-1$ para todo $n$.

\footnotetext{
†Vê-se imediatamente que as soluções $u^{D}(z)$ e $u^{N}(z)$ são equivalentes às soluções $u^{\alpha}(z)$ e $u^{\alpha^{*}}(z)$ quando $\alpha=0$.
} 
Podemos usar a constância do Wronskiano $W\left[u^{N}, u^{D}\right](n)$ e obter

$$
\frac{a_{-1}}{a_{n}}=u_{n}^{N} \bar{u}_{n+1}^{D}-u_{n+1}^{N} \bar{u}_{n}^{D} .
$$

Segue, da desigualdade de Cauchy-Schwarz, a relação

$$
\sum_{n \geq 0}\left|a_{n}\right|^{-1} \leq 2 \sqrt{\sum_{n \geq 0}\left|u_{n}^{D}\right|^{2} \sum_{n \geq 0}\left|u_{n}^{N}\right|^{2}} .
$$

Portanto, se $\sum_{n>0}\left|a_{n}\right|^{-1}=\infty$, necessariamente uma das soluções linearmente independentes $u^{\{D, N\}}(z)$ não pertence a $l^{2}\left(\mathbb{Z}_{+}, \mathbb{C}\right)$, e portanto $L$ é ponto-limite. Isto encerra a demonstração.

Mostremos que de fato existe, no caso ponto-limite, apenas uma solução de (D.1.2) quadrado-somável. O que segue é uma generalização dos resultados do Capítulo 9 de [CL] para o caso discreto e sob a condição de contorno de Dirichlet

$$
u_{-1}=0
$$

$(\alpha=0$ em (D.1.4)), que tratamos com exclusividade.

Observação D.1.4 O caso em que a condição de contorno é arbitrária $(\alpha \in(0, \pi))$ pode ser tratado como uma perturbação de posto 1 do caso com condição de contorno de Dirichlet, como ainda veremos neste apêndice.

Toda solução $\chi(z)$ de (D.1.2) é, com exceção de $u^{N}(z)$ e a menos de um múltiplo constante, da forma

$$
\chi(z)=-u^{N}(z)+m(z) u^{D}(z)
$$

(de outro modo, $\chi(z)$ seria um múltiplo constante de $u^{N}(z)$ e assim um autovetor de $L$ com autovalor $z$; isso não pode ocorrer, uma vez que $\Im z>0$ e $L$ é uma matriz hermiteana), $m(z)$ a chamada função ou coeficiente de Weyl-Titchmarsh.

Considere agora uma condição de contorno real em algum ponto $k \in \mathbb{N}$, digamos,

$$
\cos \beta u_{k}-\sin \beta a_{k} u_{k+1}=0 \text {, }
$$

$0 \leq \beta<\pi$, e questione como $m(z)$ deve ser de modo a que a solução $\chi(z)$, definida por (D.1.10), satisfaça (D.1.11); claramente, $\chi(z)$ é tal que

$$
m(z)=\frac{u_{k}^{N} \cot \beta-a_{k} u_{k+1}^{N}}{u_{k}^{D} \cot \beta-a_{k} u_{k+1}^{D}} .
$$

Se fizermos $\zeta=\cot \beta$ e mantivermos $(z, k)$ fixos, podemos reescrever (D.1.12) como

$$
m(z)=\frac{A \zeta-B}{C \zeta-D}
$$


com $A, B, C, D$ fixos enquanto $\zeta$ percorre a reta real à medida que $\beta$ vai de 0 a $\pi$. Sendo (D.1.13) uma transformação de Möbius (vide [Ahl] para detalhes), o eixo real do plano $\zeta$ tem como imagem um círculo $C_{k}$ no plano $m$. Assim, $\chi(z)$ satisfaz (D.1.11) se, e somente se, $m$ pertence a $C_{k}$.

Se reescrevermos $\zeta$ como função de $m$, vemos que a equação da imagem do eixo real (ou seja, $\Im \zeta=0$ ) é expressa por

$$
(\bar{A}-\overline{C m})(B-D m)-(A-C m)(\bar{B}-\overline{D m})=0,
$$

que é exatamente a equação do círculo $C_{k}$.

Levando em conta que

$$
\begin{gathered}
A=u_{k}^{N}(z) \quad B=a_{k} u_{k+1}^{N}(z) \\
C=u_{k}^{D}(z) \quad D=a_{k} u_{k+1}^{D}(z),
\end{gathered}
$$

é simples notar que a equação de $C_{k}$ é

$$
W[\chi, \chi](k)=0,
$$

com centro e raio dados, respectivamente, por

$$
\tilde{m}_{k}=-\frac{W\left[u^{N}, \bar{u}^{D}\right](k)}{W\left[u^{D}, u^{D}\right](k)} \quad \text { e } \quad r_{k}=\frac{1}{\left|W\left[u^{D}, u^{D}\right](k)\right|} .
$$

Uma vez que o coeficiente de $m \bar{m}$ em (D.1.14) é $W\left[u^{D}, u^{D}\right](k)$, segue que o interior de $C_{k}$ no plano $m$ é representado por

$$
\frac{W[\chi, \chi](k)}{W\left[u^{D}, u^{D}\right](k)}<0 .
$$

Temos da fórmula de Green (D.1.7) que

$$
W\left[u^{D}, u^{D}\right](k)=2 i \Im z \sum_{n=-1}^{k}\left|u_{n}^{D}\right|^{2}
$$

(já que $\left.W\left[u^{D}, u^{D}\right](-1)=0\right)$ e

$$
W[\chi, \chi](k)=2 i \Im z \sum_{n=-1}^{k}\left|\chi_{n}\right|^{2}+W[\chi, \chi](-1) .
$$

Como $W[\chi, \chi](-1)=2 i \Im\left[\left(-u_{-1}^{N}+m u_{-1}^{D}\right)\left(-\bar{u}_{0}^{N}+\bar{m} \bar{u}_{0}^{D}\right)\right]=-2 i \Im m$, (D.1.16) se torna

$$
\sum_{n=-1}^{k}\left|\chi_{n}\right|^{2}<\frac{\Im m}{\Im z}
$$


com $\Im z \neq 0$.Pontos de $m$ pertencem a $C_{k}$ se, e somente se,

$$
\sum_{n=-1}^{k}\left|\chi_{n}\right|^{2}=\frac{\Im m}{\Im z}
$$

O raio $r_{k}$ em (D.1.15) pode ser reescrito, para $\Im z>0$, como

$$
r_{k}^{-1}=2 \Im z \sum_{n=-1}^{k}\left|u_{n}^{D}\right|^{2} .
$$

Façamos agora $0<j<k<\infty, j, k \in \mathbb{N}$. Então, se $m$ esta dentro ou no círculo $C_{k}$, segue que

$$
\sum_{n=-1}^{j}\left|\chi_{n}\right|^{2}<\sum_{n=-1}^{k}\left|\chi_{n}\right|^{2} \leq \frac{\Im m}{\Im z}
$$

conseqüentemente, $m$ está contido no interior de $C_{j}$. Isto significa que $C_{j}$ contém $C_{k}$ em seu interior se $j<k$. Assim, para um dado $z, \Im z>0$, os círculos $C_{k}$ convergem ou a um círculo $C_{\infty}$, ou a um ponto $m_{\infty}$ à medida que $k \rightarrow \infty$. Se $C_{k}$ converge a um círculo, então seu raio $r_{\infty}=\lim _{k \rightarrow \infty} r_{k}$ é positivo, o que resulta, de (D.1.19), em $u^{D}(z) \in l^{2}\left(\mathbb{Z}_{+}, \mathbb{C}\right)$. Se $\hat{m}_{\infty}$ é um ponto qualquer no interior de $C_{\infty}$, segue, como vimos, que $\hat{m}_{\infty}$ está dentro de todo $C_{k}$. Logo

$$
\sum_{n=-1}^{k}\left|-u_{n}^{N}+\hat{m}_{\infty} u_{n}^{D}\right|^{2}<\frac{\Im \hat{m}_{\infty}}{\Im z}
$$

o que, no limite $k \rightarrow \infty$, nos leva a $-u^{N}(z)+\hat{m}_{\infty} u^{D}(z) \in l^{2}\left(\mathbb{Z}_{+}, \mathbb{C}\right)$. Podemos empregar o mesmo argumento quando $\hat{m}_{\infty}$ se reduz ao ponto $m_{\infty}$. Portanto, se $\Im z \neq 0$, sempre existe uma solução quadrado-somável da equação de Schrödinger (D.1.2). No caso em que $C_{k} \rightarrow C_{\infty}$, todas as soluções de (D.1.2) o são, uma vez que tanto $u^{D}(z)$ quanto $-u^{N}(z)+\hat{m}_{\infty} u^{D}(z)$ pertencem, como acabamos de ver, ao espaço $l^{2}\left(\mathbb{Z}_{+}, \mathbb{C}\right)$; esse fato nos permite identificar o caso círculo-limite (que assim é denominado por razões agora óbvias) com a existência do círculo $C_{\infty}$. Correspondentemente, temos o caso ponto-limite quando existe o ponto $m_{\infty}$. No caso $C_{k} \rightarrow m_{\infty}$ temos que $r_{\infty}=0$, que juntamente a (D.1.19) nos levam à conclusão que $u^{D}(z) \notin l^{2}\left(\mathbb{Z}_{+}, \mathbb{C}\right)$; portanto, existe nesta situação uma única solução linearmente independente de (D.1.2) quadrado-somável. É importante notar que em ambos os casos a função $\chi(z)$, definida por (D.1.10), é quadrado-somável; poderíamos, assim, definir $m(z)$ como a função que garante esse fato.

\section{D.2 Ligação entre a função $m$ e a função de Green}

Algumas das principais propriedades da função $m$ de Weyl-Titchmarsh se encontram no seguinte 
Lema D.2.1 Assumamos o caso ponto-limite. A função $m_{\infty}(z)$ definida por (D.1.10) é analítica em todo plano complexo com exceção da reta real. Mais do que isso, $m_{\infty}$ é uma função Herglotz (ou Nevanlinna), isto é, mapeia o semi-plano $\Im z>0$ em algum subconjunto seu $\left(\Im m_{\infty}>0\right.$ se $\left.\Im z>0\right)$.

A função $m_{\infty}(z)$ se relaciona com o operador resolvente (ou simplesmente resolvente), $(L-z)^{-1}$, através da fórmula

$$
\left\langle(L-z)^{-1} e_{0}, e_{0}\right\rangle=m_{\infty}(z)
$$

onde $e_{0}=\left(\begin{array}{lllll}1 & 0 & 0 & \cdots\end{array}\right)^{T}$.

Observação D.2.2 A seqüência $\left\{e_{n}\right\}_{n \geq 0}$ representa a base canônica do espaço $l^{2}\left(\mathbb{Z}_{+}, \mathbb{C}\right)$, de modo que $\left(e_{n}\right)_{m}=\delta_{m, n}{ }^{\dagger}$.

Demonstração. As propriedades analíticas da função $m_{\infty}$ seguem imediatamente da equação (D.2.1), uma vez que é possível mostrar que o elemento de matriz $\left\langle(L-z)^{-1} e_{0}, e_{0}\right\rangle$ é uma função Herglotz para qualquer matriz de Jacobi L. A equação (D.2.1) é, de fato, um caso especial da fórmula geral

$$
\left\langle(L-z)^{-1} e_{i}, e_{j}\right\rangle=\left\{\begin{array}{lll}
\frac{\chi_{i} u_{j}^{D}}{W\left[u^{D}, \bar{\chi}\right]} & \text { se } & i \geq j \\
\frac{\chi_{j} u_{i}^{D}}{W\left[u^{D}, \bar{\chi}\right]} & \text { se } & i<j
\end{array}\right.
$$

vale destacar que a expressão acima é a versão discreta da função de Green $G(i, j ; z)$, sendo (D.2.1) o caso particular $G(0,0 ; z)$. Mostremos que esta função é o núcleo integral do operador resolvente $(L-z)^{-1}$; a saber, temos a seguinte identidade (vide Capítulo 9 , Seção 2 de [CL] para a definição da função de Green associada ao problema de SturmLiouville contínuo):

$$
\sum_{k=0}^{\infty}\left(L_{i k}-z \delta_{i k}\right) G(k, j ; z)=\delta_{i j} .
$$

De fato, da definição da matriz $L$ e de (D.2.2) obtemos

$$
\sum_{k=0}^{\infty}\left(L_{i k}-z \delta_{i k}\right) G(k, j ; z)=\left\{\begin{array}{llr}
\frac{u_{j}^{D}\left(a_{i-1} \chi_{i-1}+\left(b_{i}-z\right) \chi_{i}+a_{i} \chi_{i+1}\right)}{W\left[u^{D}, \bar{\chi}\right]} & \text { se } & i>j \\
\frac{\chi_{j}\left(a_{i-1} u_{i-1}^{D}+\left(b_{i}-z\right) u_{i}^{D}+a_{i} u_{i+1}^{D}\right)}{W\left[u^{D}, \bar{\chi}\right]} & \text { se } & i<j \\
\frac{a_{i-1} u_{i-1}^{D} \chi_{i}+\left(b_{i}-z\right) u_{i}^{D} \chi_{i}+a_{i} u_{i}^{D} \chi_{i+1}}{W\left[u^{D}, \bar{\chi}\right]} & \text { se } & i=j
\end{array}\right.
$$

\footnotetext{
${ }^{\dagger} \delta_{m, n}$ é a chamada função de Kronecker, definida de tal forma que $\delta_{m, n}=0$ se $n \neq m$, e $\delta_{m, n}=1$ se $n=m$.
} 
Como $\chi(z)$ e $u^{D}(z)$ são soluções da equação de Schrödinger (D.1.2), segue que

$$
\sum_{k=0}^{\infty}\left(L_{i k}-z \delta_{i k}\right) G(k, j ; z)=\left\{\begin{array}{ccc}
0 & \text { se } & i>j \\
0 & \text { se } & i<j \\
\frac{W\left[u^{D}, \bar{\chi}\right](i)}{W\left[u^{D}, \bar{\chi}\right]}=1 & \text { se } & i=j \\
\frac{u_{0}^{D}\left(a_{1} \chi_{1}+\left(b_{0}-z\right) \chi_{0}\right)}{W\left[u^{D}, \bar{\chi}\right]}=\frac{-a_{-1} u_{0}^{D} \chi_{-1}}{W\left[u^{D}, \bar{\chi}\right]}=1 & \text { se } & i=j=0
\end{array},\right.
$$

a última identidade resultante do fato que $\chi(z)$ e $u^{D}(z)$ satisfazem, respectivamente, as condições de contorno (D.1.4) e (D.1.9). Demonstramos, portanto, a validade de (D.2.3).

Mostremos agora que $G(i, j ; z)$ é uma função analítica em $\mathbb{C} \backslash \mathbb{R}$. Para tanto, necessitamos da seguinte identidade:

$$
(L-z I)^{-1}-(L-w I)^{-1}=(z-w)(L-z I)^{-1}(L-w I)^{-1},
$$

$z, w \in \mathbb{C}$, também conhecida como primeira identidade do resolvente (ítem (a) do Teorema A.1.5). Fica claro dessa expressão que $(L-z I)^{-1}$ depende continuamente de $z$, já que se $z$ e $w$ percorrerem um conjunto compacto cuja distância ao eixo real é $d$, então

$$
\left\|(L-z I)^{-1}-(L-w I)^{-1}\right\| \leq|z-w| / d^{2}
$$

(onde usamos a desigualdade $\|L-z I\| \geq \Im z$; vide o Capítulo 5 de [Do1] para a demonstração). Em particular, toda função de Green $G(i, j ; z)$ é contínua nos semi-planos superior e inferior. Mais ainda, tais funções são analíticas, uma vez que

$$
\frac{\left\langle\left((L-z I)^{-1}-(L-w I)^{-1}\right) e_{i}, e_{j}\right\rangle}{z-w}=\left\langle(L-z I)^{-1}(L-w I)^{-1} e_{i}, e_{j}\right\rangle
$$

e se fixarmos $w$ e fizermos $z \rightarrow w$, o membro direito da identidade acima tende continuamente a $\left\langle(L-w I)^{-2} e_{i}, e_{j}\right\rangle$, independentemente da maneira como tomamos o limite. Portanto, $G(i, j ; z)$ é diferenciável com respeito a $z$, e dessa forma, analítica.

Provemos agora que $G(z) \equiv G(0,0 ; z): \mathbb{C}_{+} \mapsto \mathbb{C}_{+}$. Se $\Im z>0$, temos

$$
\begin{aligned}
\Im G(z) & =\frac{1}{2 i}\left\langle\left((L-z)^{-1}-\overline{(L-z)^{-1}}\right) e_{0}, e_{0}\right\rangle=\frac{1}{2 i}\left\langle(z-\bar{z})[(L-z)(L-\bar{z})]^{-1} e_{0}, e_{0}\right\rangle \\
& =\Im z\left\langle(L-\bar{z})^{-1} e_{0},(L-\bar{z})^{-1} e_{0}\right\rangle=\Im z\left\|(L-\bar{z})^{-1} e_{0}\right\|^{2}>0,
\end{aligned}
$$

como queríamos mostrar.

Resta-nos demonstrar a identidade (D.2.1). Sendo esta, como vimos, um caso particular de (D.2.2), obtemos

$$
G(0,0 ; z)=\frac{\chi_{0} u_{0}^{D}}{W\left[u^{D}, \bar{\chi}\right](-1)}=m_{\infty}(z)
$$

justamente (D.2.1), como queríamos. Isso conclui a demonstração do lema. 
Observação D.2.3 Existe uma demonstração alternativa de que $m_{\infty}(z): \mathbb{C}_{+} \mapsto \mathbb{C}_{+}$. Sabemos, mesmo para o caso ponto-limite, que a desigualdade (D.1.20) é satisfeita para $\hat{m}_{\infty}=m_{\infty}$. Portanto, tomando em (D.1.20) o limite $k \rightarrow \infty$, obtemos

$$
\sum_{n=0}^{\infty}\left|-u_{n}^{N}+m_{\infty} u_{n}^{D}\right|^{2}<\frac{\Im m_{\infty}}{\Im z},
$$

de onde concluímos que $\Im\left(m_{\infty}\right)>0$ caso $\Im z>0$; isso demonstra a afirmação de que $m_{\infty}$ mapeia o semi-plano complexo superior nele mesmo.

\section{D.3 Medida espectral e sua relação com comporta- mento de fronteira da função de Green}

Como veremos, as propriedades espectrais de um operador auto-adjunto (unitariamente equivalente a um operador ou matriz de Jacobi) são diretamente relacionadas ao comportamento da função de Green $G(z)$ para $z$ próximo a reta real. Se $\left\{P_{\Gamma}\right\}$ é a família de m.v.p.'s tal que $L=\int \lambda d P_{\lambda}$ (vide a Seção B.2 para as definições e demonstrações das afirmações), temos

$$
G(z)=\int_{-\infty}^{\infty} \frac{d \rho_{e_{0}}(\lambda)}{\lambda-z}
$$

onde $\rho_{e_{0}}(\lambda)=\left\langle P_{\lambda} e_{0}, e_{0}\right\rangle$ é uma função não-decrescente (pode ser constante em alguma região da reta real), contínua à direita e satisfaz os $\operatorname{limites} \lim _{\lambda \rightarrow-\infty} \rho_{e_{0}}(\lambda)=0$ e $\lim _{\lambda \rightarrow \infty}$ $\rho_{e_{0}}(\lambda)=1$. A medida de Borel-Stietjes $\mu_{e_{0}}$ gerada por $\rho_{e_{0}}(\lambda)$ (isto é, $\mu_{e_{0}}=d \rho_{e_{0}}^{\dagger}$ ) é a chamada medida espectral associada ao operador de Jacobi $L$. Sendo $e_{0}$ um vetor cíclico de $L$ em $l^{2}\left(\mathbb{Z}_{+}, \mathbb{C}\right)$ (vide a Definição B.1.2), temos que as propriedades espectrais de $L$ são completamente descritas pela medida $\mu_{e_{0}}$ (vide a Seção B.1 para os detalhes).

Observação D.3.1 De agora em diante omitimos a dependência do vetor $e_{0}$ na medida $\mu$, sendo esta subentendida. Fazemo-lo a fim de não carregar a notação.

Existe uma demonstração alternativa da identidade (D.3.1) para operadores limitados (como aqueles que estudamos) que julgamos interessante apresentar. Ela leva em conta o fato, já discutido, da função de Green ser Herglotz, e assim possuir a representação (vide o Apêndice C para a demonstração)

$$
G(z)=a z+b+\int_{-\infty}^{\infty}\left(\frac{1}{\lambda-z}-\frac{\lambda}{\lambda^{2}+1}\right) d \rho(\lambda),
$$

$\Im z>0$, onde $a \geq 0, b \in \mathbb{R}$ são constantes e $d \rho(\lambda)$ é uma medida de Borel-Stieltjes (medida espectral) que satisfaz a desigualdade

$$
\int_{-\infty}^{\infty} \frac{d \rho(\lambda)}{\lambda^{2}+1}<\infty
$$

\footnotetext{
${ }^{\dagger} \mu_{e_{0}}(B)=\int_{B} d \rho_{e_{0}}(\lambda)$ para qualquer conjunto de Borel $B$.
} 
Segue imediatamente da definição de $G(z)$ que

$$
G(z)=-z^{-1}+O\left(z^{-2}\right)
$$

no limite $z \rightarrow \infty$, o que nos permite concluir de imediato que $a=0$ em (D.3.2).

Sendo o suporte da medida espectral um conjunto compacto $\Sigma$ (já que o espectro de qualquer operador limitado é um conjunto compacto; vide o Teorema A.1.3), segue de (D.3.2) que

$$
G(z)=\left\langle(L-z)^{-1} e_{0}, e_{0}\right\rangle=b-\int_{\Sigma} \frac{\lambda}{\lambda^{2}+1} d \rho(\lambda)+\int_{\Sigma} \frac{d \rho(\lambda)}{\lambda-z} .
$$

No entanto,

$$
\int_{\Sigma} \frac{d \rho(\lambda)}{\lambda-z}=-z^{-1} \int_{\Sigma} d \rho(\lambda)+O\left(z^{-2}\right)
$$

o que nos permite concluir de (D.3.3) que

$$
b-\int_{\Sigma} \frac{\lambda}{\lambda^{2}+1} d \rho(\lambda)=0
$$

e que portanto,

$$
G(z)=\left\langle(L-z)^{-1} e_{0}, e_{0}\right\rangle=\int_{\Sigma} \frac{d \rho(\lambda)}{\lambda-z}
$$

para $\Im z>0$, e assim para todo $z \notin \Sigma$ por continuação analítica (Lema B.4 de [T]). Finalmente, observe que $\mu=d \rho$ é uma medida de probabilidade, uma vez que

$$
\left\langle L^{0} e_{0}, e_{0}\right\rangle=\int_{\Sigma} d \rho(\lambda)=1
$$

$L^{0}=I$, o operador identidade em $l^{2}\left(\mathbb{Z}_{+}, \mathbb{C}\right)$. Chegamos assim ao resultado almejado.

De grande importância nos resultados seguintes é a fórmula de inversão de BorelStieltjes ou transformada de Borel-Stieltjes inversa:

$$
\mu\left(\left(\lambda_{1}, \lambda_{2}\right)\right)+\frac{1}{2}\left[\mu\left(\left\{\lambda_{1}\right\}\right)+\mu\left(\left\{\lambda_{2}\right\}\right)\right]=\lim _{\varepsilon \downarrow 0} \frac{1}{\pi} \int_{\lambda_{1}}^{\lambda_{2}} \Im G(\lambda+i \varepsilon) d \lambda
$$

onde $\lambda_{1}<\lambda<\lambda_{2}$, cuja demonstração se encontra no Apêndice C (Lema C.5).

Nossa preocupação é com a decomposição da medida espectral $\mu$ em suas respectivas componentes absolutamente contínua e singular, $\mu=\mu_{\mathrm{ac}}+\mu_{\mathrm{s}}$, e também com a decomposição $\mu_{\mathrm{s}}=\mu_{\mathrm{pp}}+\mu_{\mathrm{sc}}$ da parte singular em suas componentes singular-contínua e puramente pontual (vide o Apêndice B para a definição destas medidas e construção dos subespaços espectrais correspondentes). Em particular, buscamos caracterizar os respectivos suportes (mínimos) das medidas $\mu, \mu_{\mathrm{ac}}, \mu_{\mathrm{pp}}$ e $\mu_{\mathrm{sc}}$. Antes de qualquer coisa, apresentemos algumas definições e propriedades relevantes ao problema. 
Definição D.3.2 Seja $\mu$ a medida de Borel-Stieltjes gerada por $\rho$ e seja $\kappa$ a medida de Lebesgue. Definimos a derivada de Radon-Nikodym de $\mu$ com respeito a $\kappa$ como

$$
\frac{d \mu}{d \kappa}(x)=\lim _{\kappa\left(I_{x}\right) \rightarrow 0}\left\{\frac{\mu\left(I_{x}\right)}{\kappa\left(I_{x}\right)}: I_{x} \text { é um intervalo de } \mathbb{R} \text { que contém } x\right\},
$$

independentemente da existência do limite.

Dizemos que a função $G(z)$ possui um limite normal em um ponto $x \in \mathbb{R}$ se $G(z)$ converge a um limite finito ou infinito à medida que $z \downarrow x$ ao longo da normal ao eixo real no ponto $x$. O seguinte resultado, uma variação do Lema 1 de $[\mathrm{GP}]$, decorre de propriedades conhecidas de mapas conformes.

Lema D.3.3 A função $G(z)$ apresenta um limite normal finito Lebesgue quase-certamente (isto é, a menos de um conjunto de medida de Lebesgue nula).

O lema seguinte está essencialmente contido no Teorema 9.1, Capítulo IV de [S].

Lema D.3.4 Seja $S$ o conjunto

$$
\{\lambda \in \mathbb{R}:(d \mu / d \kappa)(\lambda) \text { não existe finita ou infinitamente }\}
$$

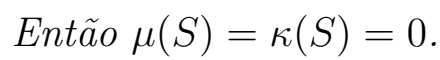

Seja $G_{+}(\lambda)=\lim _{\varepsilon \downarrow 0} G(\lambda+i \varepsilon)$ para cada $\lambda \in \mathbb{R}$ onde este limite normal existe (a menos de um conjunto de medida de Lesbesgue nula, como diz o Lema D.3.4). Seja $\Im G_{+}(\lambda)$ definido analogamente. Usando integração por partes na parte imaginária da equação (D.3.4), é possível mostrar que

Lema D.3.5 (Lema 3 de $[\mathbf{G P}])$ Se a derivada de Radon-Nikodym $(d \mu / d \nu)(\lambda)$ existir, sendo finita ou não, então $\Im G_{+}(\lambda)$ também existe e $(d \mu / d \kappa)(\lambda)=(1 / \pi) \Im G_{+}(\lambda)$.

Observação D.3.6 O Lema 3 de [GP] trata da relação entre a derivada de RadonNikodym e a função $m_{\infty}(z)$, que como veremos adiante, também é possivel em nosso caso. O Teorema B.8 de [T] traz o mesmo conteúdo do Lema D.3.5.

Voltamo-nos agora ao conceito de um suporte mínimo (também chamado de suporte essencial) de uma medida de Borel-Stieltjes.

Definição D.3.7 Um subconjunto $M$ de $\mathbb{R}$ é chamado de suporte mínimo de uma medida $\eta$ em $\mathbb{R}$ se

(i) $\eta(\mathbb{R} \backslash M)=0$, isto é, $M$ é um suporte de $\eta$;

(ii) qualquer subconjunto $M_{0} \subseteq M$ que não suporta $M$, ou seja, que $\eta\left(M_{0}\right)=0$, tem medida de Lebesgue nula $\left(\kappa\left(M_{0}\right)=0\right)$. 
Um suporte mínimo de uma medida $\eta$ dá um indicativo de onde se concentra a medida e é unicamente determinado a menos de conjuntos de $\kappa$ - e $\eta$-medida nulas. De fato, é possível mostrar que o conjunto de todos os suportes mínimos de uma dada medida $\eta$ é uma classe de equivalência sobre a relação $\sim$, definida por

$$
M \sim M^{\prime} \Leftrightarrow \kappa\left(M \triangle M^{\prime}\right)=\eta\left(M \triangle M^{\prime}\right)=0,
$$

$M, M^{\prime} \subseteq \mathbb{R}$, em que $M \triangle M^{\prime}$ representa a diferença simétrica $\left(M \backslash M^{\prime}\right) \cup\left(M^{\prime} \backslash M\right)$ (vide [GP] e as referências bibliografias nele presentes para uma demonstração desse resultado).

A seguinte classificação do espectro em termos de suportes mínimos pode ser deduzida usando o Teorema de decomposição de de la Vallée-Poussin (Teorema 9.6 do Capítulo IV de $[\mathrm{S}]$ ), o Teorema de Lebesgue-Radon-Nikodym (Teorema 6.9 de [Ru]) e o Lema D.3.5:

Lema D.3.8 (Lema 4 de [GP]) Os suportes minimos $\Sigma, \Sigma_{\mathrm{ac}}, \Sigma_{\mathrm{s}}, \Sigma_{\mathrm{sc}}$ e $\Sigma_{\mathrm{pp}}$ de $\mu$, as partes absolutamente contínua $\mu_{\mathrm{ac}}$, singular $\mu_{\mathrm{s}}$, singular-contínua $\mu_{\mathrm{sc}}$ e puramente pontual $\mu_{\mathrm{pp}}$ de $\mu$ com respeito a $\kappa$ são dados, respectivamente, por

(i) $\Sigma=\{\lambda \in E: 0<(d \mu / d \kappa)(\lambda) \leq \infty\}$,

(ii) $\Sigma_{\mathrm{ac}}=\{\lambda \in E: 0<(d \mu / d \kappa)(\lambda)<\infty\}$,

(iii) $\Sigma_{\mathrm{s}}=\{\lambda \in E:(d \mu / d \kappa)(\lambda)=\infty\}$,

(iv) $\Sigma_{\mathrm{sc}}=\{\lambda \in E:(d \mu / d \kappa)(\lambda)=\infty, \mu(\lambda)=0\}$,

(v) $\Sigma_{\mathrm{pp}}=\{\lambda \in E:(d \mu / d \kappa)(\lambda)=\infty, \mu(\lambda)>0\}$,

em que $E:=\{\lambda \in \mathbb{R}:(d \mu / d \kappa)(\lambda)$ existe $\}$.

Seja $\Sigma^{\prime}=\left\{\lambda \in E^{\prime}: 0<\Im G_{+}(\lambda) \leq \infty\right\}$, com $E^{\prime}:=\left\{\lambda \in \mathbb{R}: \Im G_{+}(\lambda)\right.$ existe $\}$. Segue, portanto, do Lema D.3.5 que $\Sigma \subseteq \Sigma^{\prime}$ (já que $\Im G_{+}(\lambda)$ pode existir sem que $(d \mu / d \kappa)$ exista, em princípio). Além do mais, se

$U=\left\{\lambda \in \mathbb{R}: \Im G_{+}(\lambda)\right.$ existe de maneira finita ou infinita, porém $(d \mu / d \kappa)(\lambda)$ não existe $\}$,

então $\Sigma^{\prime} \backslash \Sigma \subseteq U \subseteq S, S$ definido como no Lema D.3.4. Como $\mu(S)=\kappa(S)=0$, segue da definição da relação de equivalência (D.3.6) que $\Sigma^{\prime} \sim \Sigma$, e que portanto $\Sigma^{\prime}$ é um suporte mínimo de $\mu$. Resultados análogos seguem para as medidas $\mu_{\mathrm{ac}}, \mu_{\mathrm{s}}, \mu_{\mathrm{sc}}$ e $\mu_{\mathrm{pp}}$, de onde concluímos a

Proposição D.3.9 (Lema 2 de $[\mathbf{K P}]$ ) Os suportes minimos $\Sigma^{\prime}, \Sigma_{\mathrm{ac}}^{\prime}, \Sigma_{\mathrm{s}}^{\prime}, \Sigma_{\mathrm{sc}}^{\prime}$ e $\Sigma_{\mathrm{pp}}^{\prime} d e$ $\mu$, as partes absolutamente contínua $\mu_{\mathrm{ac}}$, singular $\mu_{\mathrm{s}}$, singular-contínua $\mu_{\mathrm{sc}}$ e puramente pontual $\mu_{\mathrm{pp}}$ de $\mu$ com respeito a $\kappa$ são dados, respectivamente, por

(i) $\Sigma^{\prime}=\left\{\lambda \in E^{\prime}: 0<\Im G_{+}(\lambda) \leq \infty\right\}$,

(ii) $\Sigma_{\mathrm{ac}}^{\prime}=\left\{\lambda \in E^{\prime}: 0<\Im G_{+}(\lambda)<\infty\right\}$, 
(iii) $\Sigma_{\mathrm{s}}^{\prime}=\left\{\lambda \in E^{\prime}: \Im G_{+}(\lambda)=\infty\right\}$,

(iv) $\Sigma_{\mathrm{sc}}^{\prime}=\left\{\lambda \in E^{\prime}: \Im G_{+}(\lambda)=\infty, \mu(\lambda)=0\right\}$,

(v) $\Sigma_{\mathrm{pp}}^{\prime}=\left\{\lambda \in E^{\prime}: \Im G_{+}(\lambda)=\infty, \mu(\lambda)>0\right\}$,

em que $E^{\prime}:=\left\{\lambda \in \mathbb{R}: \Im G_{+}(\lambda)\right.$ existe $\}$.

\section{D.4 Medida espectral e comportamento de fronteira da função $m$}

A identidade (D.2.1), juntamente com a representação (D.3.1), nos permitem a associação imediata entre a medida espectral $\mu$ e a função $m_{\infty}(z)$ :

$$
m_{\infty}(z)=\int_{-\infty}^{\infty} \frac{d \rho(\lambda)}{\lambda-z} .
$$

Segue, em analogia a (D.3.4), a relação

$$
\mu((a, b))+\frac{1}{2}[\mu(\{a\})+\mu(\{b\})]=\lim _{\varepsilon \downarrow 0} \frac{1}{\pi} \int_{\lambda_{1}}^{\lambda_{2}} \Im m_{\infty}(\lambda+i \varepsilon) d \lambda .
$$

Assim como na Proposição D.3.9, somos capazes de expressar os suportes mínimos $\Sigma^{\prime}$, etc., em termos do comportamento no limite à reta real da função $\Im m_{\infty}(z)$ :

Proposição D.4.1 (Proposição 1 de [GP]) Os suportes mínimos $\Sigma^{\prime \prime}, \Sigma_{\mathrm{ac}}^{\prime \prime}, \Sigma_{\mathrm{s}}^{\prime \prime}, \Sigma_{\mathrm{sc}}^{\prime \prime} e$ $\Sigma_{\mathrm{pp}}^{\prime \prime}$ de $\mu$, as partes absolutamente contínua $\mu_{\mathrm{ac}}$, singular $\mu_{\mathrm{s}}$, singular-contínua $\mu_{\mathrm{sc}} e$ puramente pontual $\mu_{\mathrm{pp}}$ de $\mu$ com respeito a $\kappa$ são dados, respectivamente, por

(i) $\Sigma^{\prime \prime}=\left\{\lambda \in E^{\prime \prime}: 0<\Im m_{\infty}^{+}(\lambda) \leq \infty\right\}$,

(ii) $\Sigma_{\mathrm{ac}}^{\prime \prime}=\left\{\lambda \in E^{\prime \prime}: 0<\Im m_{\infty}^{+}(\lambda)<\infty\right\}$,

(iii) $\Sigma_{\mathrm{s}}^{\prime \prime}=\left\{\lambda \in E^{\prime \prime}: \Im m_{\infty}^{+}(\lambda)=\infty\right\}$,

(iv) $\Sigma_{\mathrm{sc}}^{\prime \prime}=\left\{\lambda \in E^{\prime \prime}: \Im m_{\infty}^{+}(\lambda)=\infty, \mu(\lambda)=0\right\}$,

(v) $\Sigma_{\mathrm{pp}}^{\prime \prime}=\left\{\lambda \in E^{\prime \prime}: \Im m_{\infty}^{+}(\lambda)=\infty, \mu(\lambda)>0\right\}$,

em que $\Im m_{\infty}^{+}(\lambda)=\lim _{\varepsilon \downarrow 0} \Im m_{\infty}(\lambda+i \varepsilon)$ e $E^{\prime \prime}:=\left\{\lambda \in \mathbb{R}: \Im m_{\infty}^{+}(\lambda)\right.$ existe $\}$.

A análise espectral usualmente se preocupa com a identificação do espectro em detrimento aos suportes mínimos da medida espectral. A relação entre o conjunto dos suportes mínimos e o espectro é expressa pelo

Lema D.4.2 (Lema 5 de [GP]) Seja $\sigma(T)$ o espectro do operador de Schrödinger T. Então, existe um suporte mínimo $\Sigma_{\mu}$ da medida espectral $\mu$ tal que $\bar{\Sigma}_{\mu}=\sigma(T)(\bar{\Sigma}$ denota o fecho do conjunto $\Sigma$ ). 
Demonstração. Seja $\Sigma(T)$ um suporte mínimo de $\mu$ e defina $\bar{\Sigma}_{\mu}=\Sigma(T) \cap \sigma(T)$. Como $\bar{\Sigma}_{\mu} \subseteq \Sigma(T)$ e $\mu\left(\Sigma(T) \backslash \bar{\Sigma}_{\mu}\right)=0$ (vide [GP]), concluímos que $\bar{\Sigma}_{\mu}$ também é um suporte mínimo de $\mu$, pela Definição D.3.7. Sendo $\sigma(T)$ um conjunto fechado (Teorema A.1.9), segue que $\bar{\Sigma}_{\mu} \subseteq \sigma(T)$.

Considere agora o conjunto $\left(\bar{\Sigma}_{\mu}\right)^{C}=\mathbb{R} \backslash \bar{\Sigma}_{\mu}$, o complemento de $\bar{\Sigma}_{\mu}$ na reta real. Obviamente $\left(\bar{\Sigma}_{\mu}\right)^{C}$ é um aberto, e uma vez que $\bar{\Sigma}_{\mu}$ é um suporte mínimo de $\mu, \mu\left(\left(\bar{\Sigma}_{\mu}\right)^{C}\right)=$ $\mu\left(\mathbb{R} \backslash \bar{\Sigma}_{\mu}\right)=0$ pela Definição D.3.7. Temos então que $\rho(\lambda)$ é constante em $\left(\bar{\Sigma}_{\mu}\right)^{C}$, e portanto que $\bar{\Sigma}_{\mu} \supseteq \sigma(T)$ pela definição de espectro (Apêndice A). Isso completa a demonstração do lema.

É possível demonstrar resultados análogos com respeito aos espectros absolutamente contínuo e singular, que também são conjuntos fechados. Deve-se enfatizar, no entanto, que em geral os conjuntos $\Sigma_{\mathrm{ac}}, \Sigma_{\mathrm{ac}}^{\prime}$ e $\Sigma_{\mathrm{s}}, \Sigma_{\mathrm{s}}^{\prime}$ não estão contidos em seus respectivos espectros ${ }^{\dagger}$.

Os resultados que seguem dizem respeito ao comportamento dos suportes mínimos quando modificamos, por exemplo, a condição de contorno (D.1.4), e são fundamentais em todo trabalho que desenvolvemos. Denotemos por $\mu_{\mathrm{ac}}^{(\alpha)}$ e $\mu_{\mathrm{s}}^{(\alpha)}$, respectivamente, as partes absolutamente contínua e singular da medida de Borel-Stieltjes gerada por $\rho_{\alpha}(\lambda)$, e por $E_{\mathrm{ac}}(\alpha)$ e $E_{\mathrm{s}}(\alpha)$ as classes de equivalência dos suportes mínimos de $\mu_{\mathrm{ac}}^{(\alpha)}$ e $\mu_{\mathrm{s}}^{(\alpha)}$. O contraste assombroso entre os comportamentos de $\mu_{\mathrm{ac}}^{\left(\alpha_{1}\right)}$ e $\mu_{\mathrm{s}}^{\left(\alpha_{2}\right)}$ quando a condição de contorno $\alpha$ varia é exibido no próximo

Lema D.4.3 (Lema 6 de $[\mathbf{G P}]$ ) (i) $E_{\mathrm{ac}}\left(\alpha_{1}\right)=E_{\mathrm{ac}}\left(\alpha_{2}\right)$ para quaisquer condições de contorno $\alpha_{1}, \alpha_{2} \in[0, \pi)$.

(ii) Se $\mu_{\mathrm{s}}^{\left(\alpha_{1}\right)}(\mathbb{R}), \mu_{\mathrm{s}}^{\left(\alpha_{2}\right)}(\mathbb{R})>0$, então $E_{\mathrm{s}}\left(\alpha_{1}\right) \neq E_{\mathrm{s}}\left(\alpha_{2}\right)$ para quaisquer $\alpha_{1} \neq \alpha_{2}(\bmod \pi)$; além do mais, para cada par $\left\{\alpha_{1}, \alpha_{2}\right\}$ de condições de contorno diferentes existem suportes minimos $\Sigma\left(\alpha_{1}\right) \in E_{\mathrm{s}}\left(\alpha_{1}\right)$ e $\Sigma\left(\alpha_{2}\right) \in E_{\mathrm{s}}\left(\alpha_{2}\right)$ tais que $\Sigma\left(\alpha_{1}\right) \cap \Sigma\left(\alpha_{2}\right)=\emptyset$.

Demonstração. Necessitamos de uma expressão que relacione, por exemplo, as funções $m_{\infty}\left(z, \alpha_{1}\right)$ e $m_{\infty}\left(z, \alpha_{2}\right)$. De fato, como toda solução quadrado-somável, no caso pontolimite, é da forma (D.1.10) (substituímos $u^{N}(z)$ e $u^{D}(z)$, respectivamente, por $u^{\alpha_{i}}(z)$ e $\left.u^{\alpha_{i}^{*}}(z), i=1,2\right)$, temos a identidade

$$
\frac{\chi_{0}}{\chi_{-1}}=\frac{-u_{0}^{\alpha_{1}^{*}}\left(\alpha_{1}\right)+m_{\infty}\left(z, \alpha_{1}\right) u_{0}^{\alpha_{1}}\left(\alpha_{1}\right)}{-u_{-1}^{\alpha_{1}^{*}}\left(\alpha_{1}\right)+m_{\infty}\left(z, \alpha_{1}\right) u_{-1}^{\alpha_{1}}\left(\alpha_{1}\right)}=\frac{-u_{0}^{\alpha_{2}^{*}}\left(\alpha_{2}\right)+m_{\infty}\left(z, \alpha_{2}\right) u_{0}^{\alpha_{2}}\left(\alpha_{2}\right)}{-u_{-1}^{\alpha_{2}^{*}}\left(\alpha_{2}\right)+m_{\infty}\left(z, \alpha_{2}\right) u_{-1}^{\alpha_{2}}\left(\alpha_{2}\right)},
$$

de onde segue, juntamente com (D.1.6), que

$$
\frac{\sin \alpha_{1}+m_{\infty}\left(z, \alpha_{1}\right) \cos \alpha_{1}}{\cos \alpha_{1}-m_{\infty}\left(z, \alpha_{1}\right) \sin \alpha_{1}}=\frac{\sin \alpha_{2}+m_{\infty}\left(z, \alpha_{2}\right) \cos \alpha_{2}}{\cos \alpha_{2}-m_{\infty}\left(z, \alpha_{2}\right) \sin \alpha_{2}} .
$$

${ }^{\dagger}$ No entanto, estão contidos no espectro essencial; vide a Seção B.2 para a definição desse tipo de espectro. 
Obtemos, manipulando a expressão acima, a identidade

$$
m_{\infty}\left(z, \alpha_{2}\right)=\frac{1+\cot \left(\alpha_{1}-\alpha_{2}\right) m_{\infty}\left(z, \alpha_{1}\right)}{\cot \left(\alpha_{1}-\alpha_{2}\right)-m_{\infty}\left(z, \alpha_{1}\right)}
$$

exatamente como desejávamos. De (D.4.3), chegamos a

$$
\Im m_{\infty}\left(z, \alpha_{2}\right)=\frac{\Im m_{\infty}\left(z, \alpha_{1}\right)\left(1+\cot ^{2}\left(\alpha_{1}-\alpha_{2}\right)\right)}{\left(\cot \left(\alpha_{1}-\alpha_{2}\right)-\Re m_{\infty}\left(z, \alpha_{1}\right)\right)^{2}+\left(\Im m_{\infty}\left(z, \alpha_{1}\right)\right)^{2}} .
$$

Usando a notação da Proposição D.4.1, temos:

(i) $\kappa\left(\Sigma_{\mathrm{ac}}^{\prime \prime}\left(\alpha_{1}\right) \triangle \Sigma_{\mathrm{ac}}^{\prime \prime}\left(\alpha_{2}\right)\right)=0$ pela equação (D.4.4) (já que $0 \leq \Im m_{\infty}\left(z, \alpha_{1}\right)<\infty \Leftrightarrow$ $\left.0 \leq \Im m_{\infty}\left(z, \alpha_{2}\right)<\infty\right)$ e pelo Lema D.3.3. Como $\mu_{\mathrm{ac}}^{\left(\alpha_{1}\right)}$ e $\mu_{\mathrm{ac}}^{\left(\alpha_{2}\right)}$ são absolutamente contínuos com respeito à medida de Lebesgue $\kappa$ (vide a Definição B.1.14), segue que $E_{\text {ac }}\left(\alpha_{1}\right)=$ $E_{\mathrm{ac}}\left(\alpha_{2}\right)$.

(ii)

$$
\Sigma_{\mathrm{s}}^{\prime \prime}\left(\alpha_{1}\right) \cap \Sigma_{\mathrm{s}}^{\prime \prime}\left(\alpha_{2}\right)=\emptyset
$$

por (D.4.4) (já que $\Im m_{\infty}\left(z, \alpha_{1}\right)=\infty$ implica em $\Im m_{\infty}\left(z, \alpha_{2}\right)=0$ e vice-versa), com $\Sigma_{\mathrm{s}}^{\prime \prime}\left(\alpha_{1}\right) \in E_{\mathrm{s}}\left(\alpha_{1}\right)$ e $\Sigma_{\mathrm{s}}^{\prime \prime}\left(\alpha_{2}\right) \in E_{\mathrm{s}}\left(\alpha_{2}\right)$. Obtemos de (D.4.5) e da Definição D.3.7

$$
\mu_{\mathrm{s}}^{\left(\alpha_{2}\right)}\left(\Sigma_{\mathrm{s}}^{\prime \prime}\left(\alpha_{1}\right)\right)=\mu_{\mathrm{s}}^{\left(\alpha_{1}\right)}\left(\Sigma_{\mathrm{s}}^{\prime \prime}\left(\alpha_{2}\right)\right)=0,
$$

e portanto, se $\mu_{\mathrm{s}}^{\left(\alpha_{1}\right)}(\mathbb{R}), \mu_{\mathrm{s}}^{\left(\alpha_{2}\right)}(\mathbb{R})>0$, então $\Sigma_{\mathrm{s}}^{\prime \prime}\left(\alpha_{1}\right) \notin E_{\mathrm{s}}\left(\alpha_{2}\right)$ e $\Sigma_{\mathrm{s}}^{\prime \prime}\left(\alpha_{2}\right) \notin E_{\mathrm{s}}\left(\alpha_{1}\right)$. Isso encerra a demostração do lema.

O Lema D.4.3 nos mostra que enquanto o suporte mínimo da parte absolutamente contínua da medida espectral é estável por uma mudança na condição de contorno, o mesmo não ocorre com o suporte mínimo da parte singular: mais ainda, os suportes associados a medidas com duas condições de contorno distintas são ortogonais. Vale destacar que este resultado é anterior ao trabalho de Gilbert e Pearson, remontando o trabalho de Aronszajn [Ar].

Temos por fim o seguinte

Corolário D.4.4 (Corolário 1 de $[\mathrm{GP}]$ ) $O$ conjunto $S=\{\lambda \in \mathbb{R}:$ não existe uma condição de contorno $\alpha$ para a qual $\Im m_{\infty}^{+}(\lambda, \alpha)$ exista e seja igual a zero $\}$ pertence a $E_{\mathrm{ac}}(\alpha)$.

Demonstração. Pelo Lema D.4.3, é suficiente demonstrarr que $S$ é um suporte mínimo de $\mu_{\text {ac }}^{\left(\alpha_{1}\right)}$ para algum $\alpha_{1}$ fixo. Para tanto, basta mostrar que $S \sim \Sigma_{\text {ac }}^{\prime \prime}\left(\alpha_{1}\right)$, onde $\sim$ é a relação de equivalência (D.3.6), com $\eta=\mu_{\mathrm{ac}}^{\left(\alpha_{1}\right)}$.

Para quase todo $\lambda \in \Sigma_{\mathrm{ac}}^{\prime \prime}\left(\alpha_{1}\right)$ com respeito à medida de Lebesgue, $\Im m_{\infty}^{+}\left(\lambda, \alpha_{2}\right)$ existe finitamente e é maior que zero para todo $\alpha_{1} \neq \alpha_{2}(\bmod \pi)$ pelo Lema D.3.3 e pela equação (D.4.4). Portanto $\kappa\left(\Sigma_{\mathrm{ac}}^{\prime \prime}\left(\alpha_{1}\right) \backslash S\right)=0$. 
Além do mais, novamente pelo Lema D.3.3 e pela definição de $S, 0<\Im m_{\infty}^{+}\left(\lambda, \alpha_{1}\right)<\infty$ Lebesgue quase-certamente (isto é, a menos de um conjunto de medida de Lebesgue nula) em $S$. Explicitamente, $\kappa\left(S \backslash \Sigma_{\mathrm{ac}}^{\prime \prime}\left(\alpha_{1}\right)\right)=0$.

Uma vez que $\mu_{\mathrm{ac}}^{\left(\alpha_{1}\right)}$ é absolutamente contínua com respeito a $\kappa$, os resultados acima nos levam a $S \sim \Sigma_{\text {ac }}^{\prime \prime}\left(\alpha_{1}\right)$, o que demonstra o corolário. 


\section{Apêndice E}

\section{Papel da subordinância na teoria espectral}

Apresentamos nesse apêndice o conceito de solução subordinada e a conexão entre sua existência e os diversos tipos espectrais. A definição de subordinância, devida a Gilbert e Pearson (apresentada em [GP] para o problema de Sturm-Liouville contínuo e estendida para o análogo discreto por Khan e Pearson em $[\mathrm{KP}]$ ) evita a comparação pontual de soluções da equação de Schrödinger $L u=\lambda u$, especialmente difícil quando estas são oscilatórias, e nos permite relacionar o comportamento de fronteira de $m_{\infty}(z)$ para cada $\lambda \in \mathbb{R}$ ao comportamento assintótico das soluções linearmente independentes desta equação.

Um resultado anterior aos resultados de Gilbert e Pearson e devido a Hartman e Wintner (Teorema 6.4 do Capítulo XI de $[\mathrm{H}]$ ) se refere ao comportamento assintótico das soluções não-oscilatórias de $L u=\lambda u^{\dagger}$; a saber, se $L u=\lambda u$ é não-oscilatória, então sempre existe uma solução $u(r, \lambda)$, dita principal, única a menos de múltiplos escalares e que satisfaz

$$
\lim _{r \rightarrow \infty} \frac{u(r, \lambda)}{v(r, \lambda)}=0
$$

para qualquer solução linearmente independente $v(r, \lambda)$. Note que, pelo Teorema de separação de Sturm, basta uma solução de $L u=\lambda u$ ser não-oscilatória para que todas as demais o sejam.

A restrição do espectro ao intervalo $(-\infty, p)^{\ddagger}$ pode ser identificada com o conjunto

$\left\{\lambda \in(-\infty, p)\right.$ : existe uma solução $L^{2}[0, \infty)$ de $L u=\lambda u$ que satisfaz a condição de contorno\}

\footnotetext{
${ }^{\dagger}$ Uma solução $u(r, \lambda)$ é dita oscilatória em $[0, \infty)$ se, para todo $R \in \mathbb{R}_{+}$, existir um $r_{0}>R$ tal que $u(r, \lambda)$ se anula em $r=r_{0}$; soluções que não satisfazem a propriedade acima são ditas não-oscilatórias.

${ }^{\ddagger} p$ é o chamado ponto parabólico, tal que $L u=\lambda u$ é não-oscilatória para $\lambda<p$ e oscilatória caso contrário; vide Seção 3 de $[\mathrm{GP}]$ para maiores detalhes.
} 
(vide $[\mathrm{HW}]$ para a demonstração), ou com o conjunto

$\{\lambda \in(-\infty, p)$ : existe uma solução principal de $L u=\lambda u$ que satisfaz a condição de contorno\} .

A definição de uma solução subordinada estende a idéia de solução principal de tal modo que esta última tenha sentido onde as soluções são oscilatórias e mesmo quando não existam soluções quadrado-integráveis. Vale destacar que os resultados e conceitos apresentados acima podem ser estendidos para matrizes (operadores) de Jacobi (vide Capítulo 4 de $[\mathrm{T}])$.

Definição E.1 Se L for um operador de Jacobi ponto-limite, então uma solução nãotrivial u(z) da relação de recorrência (D.1.2) é dita subordinada se, e somente se,

$$
\lim _{l \rightarrow \infty} \frac{\|u(z)\|_{l}}{\|v(z)\|_{l}}=0
$$

para qualquer outra solução linearmente independente v(z) de (D.1.2), onde

$$
\|u(z)\|_{l}^{2}:=\sum_{n=0}^{[l]}\left|u_{n}\right|^{2}+(l-[l])\left|u_{[l]+1}\right|^{2}
$$

denota a norma $l^{2}\left(\mathbb{Z}_{+}, \mathbb{C}\right)$ truncada em $l \in \mathbb{R},[l]$ a parte inteira de $l$.

Observação E.2 1. Como o espaço das soluções da equação (D.1.2) possui dimensão 2, é simples observar que, para cada $z \in \mathbb{C}$ fixo, não é possível existir mais do que uma solução linearmente independente de (D.1.2) que seja subordinada. Não obstante, se para $u m z \in \mathbb{C}$ fixo existirem soluções $u(z)$ e v(z) que satisfaçam (E.3), então

$$
\lim _{l \rightarrow \infty} \frac{\|u(z)\|_{l}}{\|w(z)\|_{l}}=0
$$

para toda solução $w(z)$ linearmente independente com respeito a $u(z)$.

2. Qualquer solução de (D.1.2) pertencente a $l^{2}\left(\mathbb{Z}_{+}, \mathbb{C}\right)$ é subordinada, em particular $-u^{N}(z)+m_{\infty}(z) u^{D}(z)$.

3. Repare ainda que quando $z \equiv \lambda \in \mathbb{R}$ e a equação de Schrödinger é não-oscilatória, a Definição E.1 e a relação

$$
\lim _{n \rightarrow \infty} \frac{u_{n}}{v_{n}}=0
$$

(o análogo discreto de (E.1)) identificam o mesmo conjunto de soluções. Podemos dizer, portanto, que a existência de uma solução principal é uma condição suficiente à subordinância, sendo os conceitos equivalentes quando (D.1.2) é não-oscilatória. 
O principal progresso obtido por Gilbert e Pearson no problema de Sturm-Liouville contínuo, e posteriormente por Khan e Pearson no análogo discreto, foi estabelecer uma conexão entre a subordinância e o comportamento de fronteira de $m_{\infty}(z)$. Comecemos pelo

Teorema E.3 (Teorema 1 de $[\mathbf{K P}]$ ) Suponha, para algum $\lambda \in \mathbb{R}$, que

$$
m_{\infty}^{+}(\lambda) \equiv \lim _{\varepsilon \downarrow 0} m_{\infty}(\lambda+i \varepsilon)
$$

exista e seja real. Então, para este valor de $\lambda, \chi(\lambda) \equiv-u^{N}(\lambda)+m_{\infty}^{+}(\lambda) u^{D}(\lambda)$ é uma solução subordinada da equação de Schrödinger (D.1.2) ( $z \equiv \lambda)$.

Observação E.4 O Teorema E.3 é equivalente ao Corolário 2.(i) de [GP].

Demonstração. Apresentamos aqui somente as principais idéias envolvidas na demonstração. Esta se encontra em detalhes na Seção 3 de $[\mathrm{KP}]$.

A idéia básica é mostrar que a solução $-u^{N}(z)+m_{\infty}(z) u^{D}(z)$ é próxima a $-u^{N}(\lambda)+$ $m_{\infty}^{+}(\lambda) u^{D}(\lambda)$ na norma $\|\cdot\|_{l}$ para $z=\lambda+i \varepsilon$ e $\varepsilon$ pequeno, onde $m_{\infty}^{+}(\lambda)$ é um número real finito.

Para tanto, considere a equação

$$
u_{n}(z)=-u_{n}^{N}(\lambda)+m_{\infty}(z) u_{n}^{D}(\lambda)+a_{0}^{-1} u_{n}^{N}(\lambda) \sum_{j=0}^{n} u_{j}^{D}(\lambda) c_{j}-a_{0}^{-1} u_{n}^{D}(\lambda) \sum_{j=0}^{n} u_{j}^{N}(\lambda) c_{j},
$$

$\lambda \in \mathbb{R}, \Im z>0, n \in \mathbb{Z}_{+}$, que pode ser derivada do método da variação dos parâmetros (equação 1.51 de $[\mathrm{T}]$ ) e fornece a solução $u(z)$ da equação de recorrência inomogênea

$$
a_{n-1} u_{n-1}+\left(b_{n}-\lambda\right) u_{n}+a_{n} u_{n+1}=c_{n},
$$

$n \in \mathbb{N}$, sujeita às condições iniciais $u_{-1}=-1, u_{0}=m_{\infty}(z)$.

Tomando $c_{n}=(z-\lambda) u_{n}$, vemos que qualquer solução $u(z)$ da equação (E.5) deve ser uma solução da equação de Schrödinger (D.1.2). Com $z=\lambda+i \varepsilon$, podemos reescrever (E.5) na forma

$$
u(z)=-u^{N}(\lambda)+m_{\infty}(z) u^{D}(\lambda)+M u(z)
$$

onde o operador linear $M$ é definido pela expressão

$$
(M u(z))_{n}=a_{0}^{-1} u_{n}^{N}(\lambda) \sum_{j=0}^{n} u_{j}^{D}(\lambda) u_{j}-a_{0}^{-1} u_{n}^{D}(\lambda) \sum_{j=0}^{n} u_{j}^{N}(\lambda) u_{j} ;
$$

$M$ atua no espaço $l((0,[l]))$ das seqüências de tamanho $[l]+1$, com norma $\|\cdot\|_{l}$. Definimos em $l((0,[l]))$ uma seqüência iterativa, $\left\{u^{(n)}(z)\right\}_{n=0}^{\infty}$, de soluções aproximadas de (E.5) através das relações

$$
u^{(0)}(z)=0, \quad u^{(i+1)}(z)=-u^{N}(z)+m_{\infty}(z) u^{D}(z)+M u^{(i)}(z),
$$


cuja convergência depende de estimativas sobre a norma da matriz $M$. Tais estimativas estão diretamente relacionadas ao comportamento de $\varepsilon$ como função de $l$ (no caso, ao limite $\varepsilon(l) \rightarrow 0$ quando $l \rightarrow \infty$; para uma discussão mais detalhada vide Lema 3 de [KP] e a Seção H). É possivel mostrar que quando o limite (E.4) existe e é real, segue que

$$
\lim _{l \rightarrow \infty} \frac{\left\|-u^{N}(\lambda)+m_{\infty}(z) u^{D}(\lambda)\right\|_{l}}{\left\|u^{D}(\lambda)\right\|_{l}}=0
$$

e que portanto $-u^{N}(\lambda)+m_{\infty}(z) u^{D}(\lambda)$ é uma solução subordinada de (D.1.2), o que conclui a demonstração do Teorema.

Corolário E.5 (Corolário do Teorema 1 de $[\mathrm{KP}]$ ) Suponha, para algum $\lambda \in \mathbb{R}$, que

$$
\lim _{\varepsilon \downarrow 0}\left|m_{\infty}(\lambda+i \varepsilon)\right|=\infty
$$

Então, para esse valor de $\lambda, u^{D}(z)$ é uma solução subordinada da equação de Schrödinger (D.1.2).

Observação E.6 O Corolário E.5 é equivalente ao Corolário 2.(ii) de [GP].

Demonstração. A demonstração segue o mesmo argumento da demonstração do Teorema E.3, com pequenas modificações, de modo que a omitimos. Os detalhes podem ser encontrados na Seção 3 de $[\mathrm{KP}]$.

Enquanto o Teorema E.3 e o Coroláro E.5 fornecem condições suficientes para que exista subordinância, as condições necessárias são apresentadas no

Teorema E.7 (Teorema 2 de $[\mathbf{K P}]$ ) Suponha que, para algum $\lambda \in \mathbb{R}$, exista uma solução subordinada da equação de Schrödinger (D.1.2). Esta solução ou é um múltiplo constante de $-u^{N}(\lambda)+m u^{D}(\lambda)$, para algum $m \in \mathbb{R}$, ou um múltiplo constante de $u^{D}(\lambda)$. Podemos definir uma seqüência positiva decrescente $\left\{\varepsilon_{j}\right\}$ convergente a zero, de modo que ou

(i) $\lim _{j \rightarrow \infty} m_{\infty}\left(\lambda+i \varepsilon_{j}\right)=m$, se $-u^{N}(\lambda)+m u^{D}(\lambda)$ é subordinada,

ou

(ii) $\lim _{j \rightarrow \infty} m_{\infty}\left(\lambda+i \varepsilon_{j}\right)=\infty$, se $u^{D}(\lambda)$ é subordinada.

Observação E.8 1. Novamente apresentamos apenas as principais idéias envolvidas na demonstração. Os detalhes se encontram na Seção 3 de [KP]. O Teorema E.7 é equivalente ao Corolário 3 de $[G P]$. 
2. Repare que a função $m$ em $-u^{N}(\lambda)+m u^{D}(\lambda)$ deve ser real, pois caso contrário, $m$ e $\bar{m}$ dariam origem a duas soluções subordinadas lineramente dependentes; isso mostra que uma solução subordinada é, em um ponto real, sempre um múltiplo real de uma solução real (já que $u^{D}(\lambda)$, a outra possível solução subordinada, também é real).

Demonstração. (i) Seja $-u^{N}(\lambda)+m u^{D}(\lambda)$ uma solução subordinada de (D.1.2). Usamos novamente a estratégia empregada na demonstração do Teorema E.3, onde utilizamos a equação

$$
\begin{aligned}
u_{n}(z)=-u_{n}^{N}(\lambda)+m_{\infty}(z) u_{n}^{D}(\lambda) & +a_{0}^{-1}\left(-u_{n}^{N}(\lambda)+m u_{n}^{D}(\lambda)\right) \sum_{j=0}^{n} u_{j}^{D}(\lambda) c_{j} \\
& -a_{0}^{-1} u_{n}^{D}(\lambda) \sum_{j=0}^{n}\left(-u_{j}^{N}(\lambda)+m u_{j}^{D}(\lambda)\right) c_{j},
\end{aligned}
$$

que com $c_{n}=(z-\lambda) u_{n}$ é satisfeita por

$$
u(z)=-u^{N}(z)+m_{\infty}(z) u^{D}(z) .
$$

Escolhendo $\varepsilon=\varepsilon(l)$ de acordo com a equação $(20)$ de $[\mathrm{KP}]$, podemos deduzir, a partir da hipótese de subordinância de $-u^{N}(\lambda)+m u^{D}(\lambda)$,

$$
\lim _{l \rightarrow \infty} \frac{\left|m_{\infty}(z)-m\right|}{1+\Im m_{\infty}(z)}=0,
$$

com $z=\lambda+i \varepsilon(l)$. Desse modo, $\lim _{l \rightarrow \infty} m_{\infty}(\lambda+i \varepsilon(l))=m$, e assim a afirmação (i) do teorema segue escolhendo $\left\{\varepsilon_{j}\right\}$ como uma seqüência decrescente de $\{\varepsilon(l)\}$.

A demonstração de (ii) é semelhante a de (i), e usa as mesmas equações para $u(z)$, com exceção de algumas modificações necessárias em funções auxiliares. Novamente, a subordinância da solução $u^{D}(\lambda)$ nos conduz a

$$
\lim _{l \rightarrow \infty} \frac{\left|1 / m_{\infty}(z)\right|}{1+\Im\left(-1 / m_{\infty}(z)\right)}=0
$$

o que implica em $\lim _{l \rightarrow \infty}\left|m_{\infty}(\lambda+i \varepsilon(l))\right|=\infty$. Isso demonstra o caso (ii) do teorema.

O Teorema E.3, o Corolário E.5 e o Teorema E.7 formam um conjunto completo de condições necessárias e suficientes para a existência de uma solução subordinada da equação (D.1.2) para $z \equiv \lambda \in \mathbb{R}$, em termos do comportamento de fronteira da função $m_{\infty}(z)$ à medida que $z$ se aproxima de $\lambda$ na direção normal ao eixo real em $\lambda$. Combinando esses resultados, obtemos o

Teorema E.9 Uma solução subordinada da equação de Schrödinger (D.1.2) no ponto $\lambda \in \mathbb{R}$ existe se, e somente se, no limite $\varepsilon \downarrow 0$, ou $m_{\infty}(z)$ converge a um limite real finito, onde no caso $-u^{N}(\lambda)+m_{\infty}^{+}(\lambda) u^{D}(\lambda)$ é subordinada, ou $\left|m_{\infty}(z)\right| \rightarrow \infty$, cuja solução subordinada é $u^{D}(\lambda)$. 
Observação E.10 O Teorema E.9 é equivalente ao Teorema 1 de [GP].

Somos finalmente capazes de estabelecer uma conexão entre as propriedades espectrais da matriz de Jacobi $L$, definida a partir da equação (D.1.1), e a existência de soluções subordinadas da equação de Schrödinger (D.1.2) $(z \equiv \lambda \in \mathbb{R})$. Notando que $u^{D}(\lambda)$ é uma solução de (D.1.2) que satisfaz a condição de contorno (D.1.9) em $n=-1$, temos o

Teorema E.11 (Teorema 3 de [GP]) Seja L o operador de Jacobi definido em $l^{2}\left(\mathbb{Z}_{+}, \mathbb{C}\right)$ e representado por (D.1.1), e seja $\mu=d \rho$ a medida espectral de $T$, como na equação (D.3.1). Os suportes mínimos $\Sigma^{\prime \prime \prime}, \Sigma_{\mathrm{ac}}^{\prime \prime \prime}, \Sigma_{\mathrm{s}}^{\prime \prime \prime}, \Sigma_{\mathrm{sc}}^{\prime \prime \prime}$ e $\Sigma_{\mathrm{pp}}^{\prime \prime \prime}$ de $\mu$, das partes absolutamente contínua $\mu_{\mathrm{ac}}$, singular $\mu_{\mathrm{s}}$, singular-contínua $\mu_{\mathrm{sc}}$ e puramente pontual $\mu_{\mathrm{pp}}$ de $\mu$ com respeito a $\kappa$ são dados, respectivamente, por

(i) $\Sigma^{\prime \prime \prime}=\mathbb{R} \backslash \Sigma_{0}, \quad \Sigma_{0}:=\{\lambda \in \mathbb{R}$ : existe uma solução subordinada da equação de Schrödinger (D.1.2), porém esta não satisfaz a condição de contorno (D.1.9)\},

(ii) $\Sigma_{\text {ac }}^{\prime \prime \prime}=\{\lambda \in \mathbb{R}:$ não existe uma solução subordinada da equação de Schrödinger (D.1.2)\},

(iii) $\Sigma_{\mathrm{s}}^{\prime \prime \prime}=\{\lambda \in \mathbb{R}$ : existe uma solução subordinada da equação de Schrödinger (D.1.2) que satisfaz a condição de contorno (D.1.9)\},

(iv) $\Sigma_{\mathrm{sc}}^{\prime \prime \prime}=\{\lambda \in \mathbb{R}$ : existe uma solução subordinada da equação de Schrödinger (D.1.2) que satisfaz a condição de contorno (D.1.9), mas esta não pertence a $\left.l^{2}\left(\mathbb{Z}_{+}, \mathbb{C}\right)\right\}$,

(v) $\Sigma_{\mathrm{pp}}^{\prime \prime \prime}=\{\lambda \in \mathbb{R}:$ existe uma solução subordinada da equação de Schrödinger (D.1.2) que satisfaz a condição de contorno (D.1.9) e que pertence a $\left.l^{2}\left(\mathbb{Z}_{+}, \mathbb{C}\right)\right\}$.

Observação E.12 1. O Teorema E.11 combina tanto elementos do Teorema 3 de [KP] quanto elementos do Teorema 3 de [GP].

2. (i) é equivalente a $\mu\left(\Sigma_{0}\right)=\kappa\left(\Sigma_{0}\right)=0$, pela definição de suporte mínimo.

Demonstração. Precisamos apenas mostrar (ii) e (iii), e uma vez que (v) é conhecido, (iii) e (v) implicam (iv) (o espectro singular-contínuo é o complemento do espectro puramente pontual com respeito à parte singular do espectro) e (ii) e (iii) implicam (i).

(ii) Este segue imediatamente do Corolário D.4.4 (restrito ao caso particular $\alpha=0$ ) e do Teorema E.9, que diz que existe uma solução subordinada se, e somente se, $\Im m_{\infty}^{+}(\lambda)=$ 0 ou $\Im m_{\infty}^{+}(\lambda)=\infty$.

(iii) É suficiente demonstrarmos que $\Sigma_{\mathrm{s}}^{\prime \prime \prime} \sim \Sigma_{\mathrm{s}}^{\prime \prime}$, onde $\sim$ é a relação de equivalência definida por (D.3.6) com $\eta=\mu$. Como o conjunto

$$
S=\left\{\lambda \in \mathbb{R}: \lim _{\varepsilon \downarrow 0}\left|m_{\infty}(\lambda+i \varepsilon)\right|=\infty, \lim _{\varepsilon \downarrow 0} \Im m_{\infty}(\lambda+i \varepsilon) \neq \infty\right\}
$$


tem medida de Lebesgue nula (pelo Lema D.3.3) e $\mu(S)=0$, temos da Proposição D.3.9 que

$$
\Sigma_{\mathrm{s}}^{\prime \prime \prime \prime}=\left\{\lambda \in \mathbb{R}: \lim _{\varepsilon \downarrow 0}\left|m_{\infty}(\lambda+i \varepsilon)\right|=\infty\right\} \sim \Sigma_{\mathrm{s}}^{\prime \prime}
$$

e que portanto $\Sigma_{\mathrm{s}}^{\prime \prime \prime} \sim \Sigma_{\mathrm{s}}^{\prime \prime}$ é equivalente a $\Sigma_{\mathrm{s}}^{\prime \prime \prime \prime} \sim \Sigma_{\mathrm{s}}^{\prime \prime \prime}$.

Segue do ítem (ii) do Teorema E.7 e da relação (E.7) a inclusão $\Sigma_{\mathrm{s}}^{\prime \prime \prime \prime} \subseteq \Sigma_{\mathrm{s}}^{\prime \prime \prime}$. Não obstante, a solução $u^{D}(\lambda)$ é uma solução subordinada da equação (D.1.2) para qualquer $\lambda \in \Sigma_{\mathrm{s}}^{\prime \prime \prime}$, o que implica, pelo Teorema E.9, que $\lambda \in \Sigma_{\mathrm{s}}^{\prime \prime \prime \prime}$. Portanto, $\Sigma_{\mathrm{s}}^{\prime \prime \prime}=\Sigma_{\mathrm{s}}^{\prime \prime \prime \prime}$, e assim concluímos a demonstração do teorema.

Observação E.13 1. Vemos que quando L é ponto-limite, podemos entender as soluções subordinadas como uma extensão das soluções $l^{2}\left(\mathbb{Z}_{+}, \mathbb{C}\right)$. De fato, a relação entre as soluções subordinadas e os suportes mínimos da parte singular da medida espectral é idêntica à relação entre as soluções $l^{2}\left(\mathbb{Z}_{+}, \mathbb{C}\right)$ e os suportes mínimos da parte puramente pontual da medida espectral, como evidenciado pelo Teorema E.11.

2. Grosseiramente, a inexistência de soluções subordinadas indica que qualquer par de soluções linearmente independentes da equação de Schrödinger (D.1.2) apresentam comportamentos assintóticos similares (por exemplo, duas soluções oscilatórias que diferem apenas por uma fase), independente da condição de contorno em $n=-1$.

3. Já a existência de soluções subordinadas indica a presença de uma "hierarquia" entre as soluções linearmente independentes de (D.1.2) (soluções com distintas taxas de crescimento), conceito explorado por Jitomirskaya e Last em [JL]; discutimos os principais resultados deste trabalho no Apêndice $H$. 



\section{Apêndice F}

\section{Ferramentas utilizadas na determinação da natureza espectral}

Apresentamos nesse apêndice as definições de matriz de transferência e das variáveis de Prüfer, bem como discutimos a importância destas grandezas na determinação da natureza espectral por meio dos critérios de Last e Simon presentes em [LS1].

\section{F.1 Definição da matriz de transferência}

Definimos por

$$
T(n, n-1 ; z)=\left(\begin{array}{cc}
\frac{z-b_{n}}{a_{n}} & -\frac{a_{n-1}}{a_{n}} \\
1 & 0
\end{array}\right)
$$

com $z \in \mathbb{C}$ e $n \in \mathbb{Z}_{+}$, a matriz de transferência associada a uma solução no espaço de Hilbert $\mathcal{H} \cong l^{2}\left(\mathbb{Z}_{+}, \mathbb{C}\right)$ da equação de Schrödinger (D.1.2); em outras palavras, definimos $T(n, n-1 ; z)$ através da relação

$$
\left(\begin{array}{c}
u_{n+1} \\
u_{n}
\end{array}\right)=T(n, n-1 ; z)\left(\begin{array}{c}
u_{n} \\
u_{n-1}
\end{array}\right)
$$

$\left\{u_{n}(z)\right\}_{n=0}^{\infty}$ a solução de (D.1.2) a qual nos referimos.

Compreendemos o significado da nomenclatura "matriz de transferência" quando observamos (F.1.2): conhecidas as soluções nos pontos $n-1$ e $n$ e a matriz $T(n, n-1 ; z)$, somos capazes de obter a solução no ponto $n+1$ para todo $n \in \mathbb{N}$.

Observação F.1.1 A definição de matriz de transferência no contexto de equações de Schrödinger contínuas se encontra em [LS1] e nas referências bibliográficas lá presentes. 
Outra grandeza bastante útil é o produto das $n+1$ primeiras matrizes de transferência, denotado por

$$
T(n ; z)=T(n, n-1 ; z) T(n-1, n-2 ; z) \cdots T(0,-1 ; z) ;
$$

a saber, dado um par arbitrário de condições iniciais (como (D.1.5) e (D.1.6), por exemplo), construímos qualquer solução da equação de Schrödinger (D.1.2) a partir da aplicação de $T(n ; z), n \in \mathbb{Z}_{+}$, sobre estas condições:

$$
\left(\begin{array}{c}
u_{n+1} \\
u_{n}
\end{array}\right)=T(n ; z)\left(\begin{array}{c}
u_{0} \\
u_{-1}
\end{array}\right) .
$$

Observação F.1.2 A matriz $T(n ; z)$ também será chamada de matriz de transferência nesse trabalho.

\section{F.2 Critérios de Last e Simon}

O comportamento da matriz de transferência é um reflexo do comportamento dos autovetores de (D.1.2). De fato, se adotarmos, por exemplo, (D.1.6) como condições iniciais ortogonais, podemos reescrever, graças à (F.1.4), a matriz de transferência $T(n ; z)$ como

$$
T(n ; z)=\left(\begin{array}{cc}
u_{n+1}^{\alpha^{*}} & u_{n+1}^{\alpha} \\
u_{n}^{\alpha^{*}} & u_{n}^{\alpha}
\end{array}\right),
$$

onde $\alpha^{*}=\alpha+\pi / 2 \mathrm{e}$

$$
T(0 ; z)=\left(\begin{array}{cc}
\cos \alpha & \sin \alpha \\
-\sin \alpha & \cos \alpha
\end{array}\right) \text {. }
$$

Dessa forma, $\frac{1}{N} \sum_{n=0}^{L}\|T(n ; z)\| \mathrm{e} \frac{1}{N} \sum_{n=0}^{N+1}\left[\left|u_{n}^{\alpha^{*}}\right|+\left|u_{n}^{\alpha}\right|\right]$ são grandezas comparáveis. Last e Simon, no seminal trabalho [LS1], exploraram esta conexão, e a partir dos resultados de subordinância apresentados na Seção E, desenvolveram critérios de classificação espectral considerando o comportamento assintótico da norma da matriz de transferência $T(n ; \lambda), \lambda \in \sigma_{\text {ess }}(L)\left(\sigma_{\text {ess }}(L)\right.$ é o espectro essencial da matriz de Jacobi $L$ definida por (D.1.1)).

Essas são técnicas deveras poderosas e úteis ao nosso trabalho, já que temos um controle muito maior do comportamento da matriz de transferência do que da função $m$ de Weyl-Titchmarsh, em geral bastante difícil de se estimar.

Antes de apresentarmos os critérios referidos acima, mostremos algumas estimativas baseadas na teoria de subordinância descrita no Apêndice E.

Observação F.2.1 Os resultados que seguem são todos baseados ou extraídos de [LS1], com apenas algumas modificações. 
Fixemos $\lambda$. Para cada $\alpha \in[0, \pi)$, seja $\Phi_{\alpha}$ o vetor bidimensional associado à solução de (D.1.2) que satisfaz o par de condições iniciais $(\sin \alpha, \cos \alpha)$, isto é,

$$
\Phi_{\alpha}(n)=\left(\begin{array}{c}
u_{n+1}^{\alpha} \\
u_{n}^{\alpha}
\end{array}\right)=T(n, \lambda)\left(\begin{array}{c}
\sin \alpha \\
\cos \alpha
\end{array}\right) ;
$$

seja $\Psi_{\alpha}=\Phi_{\alpha^{*}}(n)$ (vide (D.1.6) e a discussão procedente), de tal forma que

$$
\Psi_{\alpha}(n)=\left(\begin{array}{c}
u_{n+1}^{\alpha^{*}} \\
u_{n}^{\alpha^{*}}
\end{array}\right)=T(n, \lambda)\left(\begin{array}{c}
\cos \alpha \\
-\sin \alpha
\end{array}\right) .
$$

Como sabemos, o Wronskiano de $u^{\alpha^{*}}(\lambda)$ e $u^{\alpha}(\lambda)$ é constante; a saber, $W\left[u^{\alpha^{*}}, u^{\alpha}\right](-1)=$ $\sin ^{2} \alpha+\cos ^{2} \alpha=\langle\Phi, B \Psi\rangle=1, \operatorname{com}$

$$
B=\left(\begin{array}{cc}
0 & 1 \\
-1 & 0
\end{array}\right)
$$

Segue, portanto, da desigualdade de Cauchy-Schwarz que

$$
\left\|\Phi_{\alpha}(n)\right\|\left\|\Psi_{\alpha}(n)\right\| \geq 1 .
$$

Claramente, $\left\|\Psi_{\alpha}(n)\right\| \leq\|T(n ; \lambda)\|$ graças à definição (F.2.3), de modo que

$$
\frac{1}{N} \sum_{n=0}^{N}\left\|\Psi_{\alpha}(n)\right\|^{2} \leq \frac{1}{N} \sum_{n=0}^{N}\|T(n ; \lambda)\|^{2} .
$$

De (F.2.4)

$$
1 \leq\left(\frac{1}{N} \sum_{n=0}^{N}\left\|\Phi_{\alpha}(n)\right\|\left\|\Psi_{\alpha}(n)\right\|\right)^{2} \leq\left(\frac{1}{N} \sum_{n=0}^{N}\left\|\Phi_{\alpha}(n)\right\|^{2}\right)\left(\frac{1}{N} \sum_{n=0}^{N}\left\|\Psi_{\alpha}(n)\right\|^{2}\right),
$$

(novamente pela desigualdade de Cauchy-Schwarz), que conjuntamente a (F.2.5) nos leva ao

\section{Lema F.2.2 (Lema 3.1 de [LS1])}

$$
\frac{\sum_{n=0}^{N}\left\|\Psi_{\alpha}(n)\right\|^{2}}{\sum_{n=0}^{N}\left\|\Phi_{\alpha}(n)\right\|^{2}} \leq\left(\frac{1}{N} \sum_{n=0}^{N}\|T(n ; \lambda)\|^{2}\right)^{2} .
$$

Tendo em mãos o Lema F.2.2, somos capazes de obter o

Teorema F.2.3 (Teorema 3.2 de [LS1]) Se a equação de Schrödinger (D.1.2) possui uma solução subordinada em $z=\lambda \in \mathbb{R}$, então

$$
\lim _{N \rightarrow \infty} \frac{1}{N} \sum_{n=0}^{N}\|T(n ; \lambda)\|^{2}=\infty .
$$


Demonstração. O teorema segue quase diretamente do Lema F.2.2 e da definição de solução subordinada. Para tanto, devemos reescrever a desigualdade (F.2.6) em termos das soluções linearmente independentes $u^{\alpha}(\lambda)$ (que suporemos subordinada) e $u^{\alpha^{*}}(\lambda)$. Temos das definições de $\Phi_{\alpha}(n)$ e de $\Psi_{\alpha}(n)$ (equações (F.2.2) e (F.2.3), respectivamente)

$$
\sum_{n=0}^{N+1}\left|u_{n}^{\alpha^{*}}\right|^{2} \leq \sum_{n=0}^{N}\left\|\Psi_{\alpha}(n)\right\|^{2}
$$

e

$$
\sum_{n=0}^{N+1}\left|u_{n}^{\alpha}\right|^{2} \geq \frac{1}{2} \sum_{n=0}^{N}\left(\left|u_{n}^{\alpha}\right|^{2}+\left|u_{n+1}^{\alpha}\right|^{2}\right) \geq \frac{1}{2} \sum_{n=0}^{N}\left\|\Phi_{\alpha}(n)\right\|^{2}
$$

de tal modo que

$$
\frac{\sum_{n=0}^{N+1}\left|u_{n}^{\alpha^{*}}\right|^{2}}{\sum_{n=0}^{N+1}\left|u_{n}^{\alpha}\right|^{2}} \leq 2 \frac{\sum_{n=0}^{N}\left\|\Psi_{\alpha}(n)\right\|^{2}}{\sum_{n=0}^{N}\left\|\Phi_{\alpha}(n)\right\|^{2}} .
$$

Obtemos, da definição de solução subordinada (E.3) e das desigualdades (F.2.6), (F.2.9),

$$
\lim _{N \rightarrow \infty}\left(\frac{1}{N} \sum_{n=0}^{N}\|T(n ; \lambda)\|^{2}\right)^{2} \geq \lim _{N \rightarrow \infty} \frac{\sum_{n=0}^{N+1}\left|u_{n}^{\alpha^{*}}\right|^{2}}{\sum_{n=0}^{N+1}\left|u_{n}^{\alpha}\right|^{2}}=\infty
$$

de onde segue (F.2.7), como queríamos mostrar.

O Teorema F.2.3 nos mostra que a existência de uma solução subordinada da equação (D.1.2), para um $z=\lambda \in \mathbb{R}$ fixo, necessariamente acarreta no crescimento da norma da matriz de transferência associada. Isso porque a norma da matriz de transferência herda o crescimento do maior autovetor da equação de Schrödinger, que na presença de uma solução subordinada (decrescente) deve crescer (graças à constância do Wronskiano). Esta questão é discutida em detalhes no Apêndice H.

Definamos o conjunto

$$
Q_{1}:=\left\{\lambda \in \mathbb{R}: \lim _{N \rightarrow \infty} \frac{1}{N} \sum_{n=0}^{N}\|T(n ; \lambda)\|^{2}=\infty\right\} ;
$$

então, de acordo com o Teorema F.2.3, segue que $\Sigma_{0} \cup \Sigma_{\mathrm{s}}^{\prime \prime \prime} \subseteq Q_{1}$, com $\Sigma_{0}$, $\Sigma_{\mathrm{s}}^{\prime \prime \prime}$ definidos como no Teorema E.11. Caso admitamos que a solução subordinada satisfaz a condição de contorno em $n=-1$ (isto é, $u^{D}(\lambda)$ é a solução subordinada para $L$ satisfazendo a condição de contorno de Dirichlet (D.1.9)), então o Teorema F.2.3 nos fornece uma condição necessária à existência de espectro singular.

Temos no sentido complementar ao Teorema F.2.3 o seguinte 
Teorema F.2.4 (Teorema 1.1 de [LS1]) Seja L a matriz de Jacobi (D.1.1) associada ao operador auto-adjunto $T$, definido no espaço de Hilbert $\mathcal{H} \cong l^{2}\left(\mathbb{Z}_{+}, \mathbb{C}\right)$ e que satisfaz a condição de contorno (D.1.9). Seja

$$
Q_{0}=\left\{\lambda: \liminf _{N \rightarrow \infty} \frac{1}{N} \sum_{n=0}^{N}\|T(n ; \lambda)\|^{2}<\infty\right\} .
$$

Então $Q_{0}$ é um suporte mínimo da parte absolutamente contínua da medida espectral $\mu$ e $Q_{0}$ possui medida nula com respeito à parte singular da medida espectral.

Demonstração. O Teorema F.2.3 nos diz que $\Sigma_{0} \cup \Sigma_{\mathrm{s}}^{\prime \prime \prime} \subseteq Q_{1} \subset \mathbb{R} \backslash Q_{0}$, de modo que $Q_{0} \subset \mathbb{R} \backslash\left(\Sigma_{0} \cup \Sigma_{\mathrm{s}}^{\prime \prime \prime}\right)$. Como $\mathbb{R} \backslash\left(\Sigma_{0} \cup \Sigma_{\mathrm{s}}^{\prime \prime \prime}\right)=\Sigma_{\text {ac }}^{\prime \prime \prime}$ é o suporte mínimo da parte absolutamente contínua, $\mu_{\mathrm{ac}}$, da medida espectral $\mu$ (resultado decorrente do Teorema E.11), a inclusão $Q_{0} \subset \Sigma_{\text {ac }}^{\prime \prime \prime}$ implica em $\mu_{\mathrm{ac}}(A)>0$ caso $A \subset Q_{0}$ e $\kappa(A)>0$. Resta-nos demonstrar a inclusão $\Sigma_{\mathrm{ac}}^{\prime \prime \prime} \subset Q_{0}$. Para tanto, necessitamos da

Proposição F.2.5 (Proposição 3.3 de [LS1]) $\lambda \in Q_{0}$ para quase todo $\lambda$ com respeito à medida espectral $\mu_{a c}$ (isto é, a menos de um conjunto de $\mu_{a c}$-medida nula).

Demonstração. Defina a medida $\mu(\lambda)=\min \left(\mu^{D}, \mu^{N}\right)$, onde $\mu^{D}$ e $\mu^{N}$ representam as medidas espectrais dos operadores que satisfazem, respectivamente, as condições de contorno (D.1.9) e $u_{-1}=1$, e

$$
\min \left(\mu_{1}, \mu_{2}\right)(S)=\inf _{A, B: S \subset A \cup B}\left\{\mu_{1}(A)+\mu_{2}(B)\right\} .
$$

Sendo as partes singulares de $\mu^{D}$ e $\mu^{N}$ disjuntas, e as partes absolutamente contínuas equivalentes, pelo Lema D.4.3, segue que $\mu$ é equivalente a $\mu_{\mathrm{ac}}^{D}$.

Necessitamos de alguns outros ingredientes para concluir a demonstração. Como

$$
\lim _{\varepsilon \downarrow 0} \frac{1}{\pi} \Im G(n, n ; \lambda+i \varepsilon)=\left|\chi_{n}(\lambda)\right|^{2} d \mu^{D}(\lambda),
$$

pelo Teorema 2.3 de [LS1], segue que $\left|\chi_{n}(\lambda)\right|^{2} d \mu^{D}(\lambda)$ é a medida espectral de $L$ com vetor (cíclico) $e_{n}$. Logo, obtemos do Teorema espectral (B.2.6),

$$
\int\left|\chi_{n}(\lambda)\right|^{2} d \mu^{D}(\lambda)=\left\langle e_{n}, e_{n}\right\rangle=1
$$

(Teorema 2.1D de [LS1]). Temos, a partir de (F.2.1) (com $\alpha=0)$, (F.2.12), e das considerações anteriores, a desigualdade

$$
\int d \mu_{\mathrm{ac}}^{D}(\lambda)\|T(n ; \lambda)\|^{2} \leq 4
$$

válida para todo $n \in \mathbb{Z}_{+}$, o que nos permite concluir

$$
\int d \mu_{\mathrm{ac}}(\lambda) G_{N}(\lambda) \leq C,
$$


onde $G_{N}(\lambda)=\frac{1}{N} \sum_{n=0}^{N}\|T(n ; \lambda)\|^{2}$ e $C \leq 4$.

Obtemos, da desigualdade (F.2.13) e do Lema de Fatou (Lema 1.28 de [Ru])

$$
\liminf _{N \rightarrow \infty} \int d \mu_{\mathrm{ac}}(\lambda) G_{N}(\lambda) \leq \int d \mu_{\mathrm{ac}}(\lambda) \liminf _{N \rightarrow \infty} G_{N}(\lambda)<\infty,
$$

e portanto, $\liminf _{N \rightarrow \infty} G_{N}(\lambda)<\infty$ quase certamente com respeito à medida $d \mu_{\mathrm{ac}}(\lambda)$. Isso encerra a demonstração da proposição.

Obtemos da Proposição F.2.5 a inclusão $\Sigma_{\text {ac }}^{\prime \prime \prime} \subset Q_{0}$, o que encerra a demonstração do teorema.

O Teorema F.2.4 estabelece um critério, ou seja, uma condição necessária e suficiente $\left(\liminf _{N \rightarrow \infty} G_{N}(\lambda)<\infty\right)$ à existência de espectro absolutamente contínuo. Repare que esta condição impõe uma limitação ao crescimento dos autovetores de (D.1.2); temos, por exemplo, das relações (F.2.5) e (F.2.8) que

$$
\liminf _{N \rightarrow \infty} \sum_{n=0}^{N+1}\left|u_{n}^{\alpha^{*}}\right|^{2} \leq \liminf _{N \rightarrow \infty} \sum_{n=0}^{N}\left\|\Psi_{\alpha}(n)\right\|^{2} \leq \liminf _{N \rightarrow \infty} G_{N}(\lambda)<\infty,
$$

o que demonstra nossa afirmação (um resultado análogo pode ser demonstrado para a solução ortogonal $u^{\alpha}(\lambda)$ ). Esse resultado vai de encontro com a afirmação de que a inexistência de soluções subordinadas (condição que define o suporte mínimo da parte absolutamente contínua do espectro, pelo Teorema E.11) resulta no mesmo comportamento assintótico entre os autovetores (vide a Observação E.13).

Temos como resultado direto do Teorema F.2.4 o seguinte

Teorema F.2.6 Suponha que a matriz de transferência $T(n ; \lambda)$ associada à equação de Schrödinger (D.1.2) satisfaça a relação $\lim _{N \rightarrow \infty} \frac{1}{N} \sum_{n=0}^{N}\|T(n ; \lambda)\|^{-2}=\infty$ para algum $\lambda \in \mathbb{R}$. Então, existe uma solução subordinada $u(\lambda)$ da equação $L u=\lambda u$.

Observação F.2.7 O Teorema F.2.6 fornece uma condição suficiente à existência de uma solução subordinada da equação de Schrödinger (D.1.2), sendo assim um complemento ao Teorema F.2.3, que como vimos, estabelece uma condição necessária à existência de tal solução.

Demonstração. Suponha que exista um $\lambda \in \mathbb{R}$ tal que a relação $\lim _{N \rightarrow \infty} \frac{1}{N} \sum_{n=0}^{N}\|T(n ; \lambda)\|^{2}$ $=\infty$ seja satisfeita. Como

$$
\lim _{N \rightarrow \infty} \frac{1}{N} \sum_{n=0}^{N}\|T(n ; \lambda)\|^{2}=\infty \quad \Rightarrow \quad \liminf _{N \rightarrow \infty} \frac{1}{N} \sum_{n=0}^{N}\|T(n ; \lambda)\|^{2}=\infty,
$$

pela definição de limite (vide p.14 de $[\mathrm{Ru}]$ ), segue que $\lambda \in\left(Q_{0}\right)^{C}, Q_{0}$ como em (F.2.11). Concluímos, do Teorema F.2.4, que $\lambda \in\left(Q_{0}\right)^{C}=\left(\Sigma_{\text {ac }}^{\prime \prime \prime}\right)^{C}=\Sigma_{0} \cup \Sigma_{\mathrm{s}}^{\prime \prime \prime}$, e que portanto existe uma solução subordinada da equação $L u=\lambda u$, pela definição dos suportes $\Sigma_{0}$ e $\Sigma_{\mathrm{s}}^{\prime \prime \prime}$. 
Observação F.2.8 Como $Q_{1} \supseteq \Sigma_{0} \cup \Sigma_{s}^{\prime \prime \prime}$, pelo Teorema F.2.3 e $Q_{1} \subseteq \Sigma_{0} \cup \Sigma_{s}^{\prime \prime \prime}$, pelo Teorema F.2.6, temos que $Q_{1}=\Sigma_{0} \cup \Sigma_{s}^{\prime \prime \prime}$, e portanto $Q_{1}=\{\lambda \in \mathbb{R}$ : existe uma solução subordinada da equação de Schrödinger (D.1.2)\} (lembre-se que $Q_{1}$ é definido por (F.2.10)).

Diferentemente do que ocorre com o espectro absolutamente contínuo, não somos capazes de obter suportes mínimos para as partes singular-contínua e puramente pontual do espectro. Existem, no entanto, alguns resultados que nos permitem delinear tais conjuntos. O primeiro deles se deve a Simon e Stolz [SS], e afirma que

Teorema F.2.9 (Teorema 2.1 de $[\mathrm{SS}]$ ) Suponha que a matriz de transferência $T(n ; \lambda)$ associada à equação de Schrödinger (D.1.2) satisfaça

$$
\sum_{n=0}^{\infty}\|T(n ; \lambda)\|^{-2}=\infty
$$

para algum $\lambda \in \mathbb{R}$. Então, a equação Lu $=\lambda u$ não apresenta uma solução $l^{2}\left(\mathbb{Z}_{+}, \mathbb{C}\right)$.

Observação F.2.10 O Teorema F.2.9 é, na realidade, a versão para matrizes de Jacobi do Teorema 2.1 de [SS]

Demonstração. Sendo $T(n ; \lambda)$ uma matriz unimodular de dimensão 2 (já que obtemos de (F.2.1) a identidade $\operatorname{det} T(n ; \lambda)=W\left[u^{\alpha_{*}}, u^{\alpha}\right](n)=W\left[u^{\alpha_{*}}, u^{\alpha}\right](-1)=1$ para todo $n \in \mathbb{Z}_{+}$), sabemos que

$$
\left\|T(n ; \lambda)^{-1}\right\|=\|T(n ; \lambda)\|
$$

Se $u(\lambda)$ é uma solução qualquer da equação de Schrödinger (D.1.2), temos de (F.1.4)

$$
\left(\begin{array}{c}
u_{0} \\
u_{-1}
\end{array}\right)=T(n ; z)^{-1}\left(\begin{array}{c}
u_{n+1} \\
u_{n}
\end{array}\right)
$$

o que, conjuntamente à identidade (F.2.16), nos leva a desigualdade

$$
\left|u_{n+1}\right|^{2}+\left|u_{n}\right|^{2} \geq\left(\left|u_{0}\right|^{2}+\left|u_{-1}\right|^{2}\right)\|T(n ; \lambda)\|^{-2} .
$$

Obtemos de (F.2.17) e da hipótese (F.2.15) o resultado

$$
2 \sum_{n=0}^{\infty}\left|u_{n}\right|^{2} \geq\left(\left|u_{0}\right|^{2}+\left|u_{-1}\right|^{2}\right) \sum_{n=0}^{\infty}\|T(n ; \lambda)\|^{-2}=\infty,
$$

que nos permite concluir que a equação de Schrödinger $L u=\lambda u$ não possui soluções $l^{2}\left(\mathbb{Z}_{+}, \mathbb{C}\right)$, já que $u(\lambda)$ é uma solução arbitrária desta mesma equação.

Definamos o cojunto

$$
Q_{2}:=\left\{\lambda \in \mathbb{R}: \sum_{n=0}^{\infty}\|T(n ; \lambda)\|^{-2}=\infty\right\} .
$$


A conclusão mais imediata que tiramos do Teorema F.2.9 é que se nos restringirmos ao espectro essencial de $\mu$, obtemos $Q_{2} \subset\left(\Sigma_{\mathrm{pp}}^{\prime \prime \prime}\right)^{C}$, a menos de um conjunto de medida espectral $\mu$ nula (em princípio, o conjunto $\Sigma_{0}$ poderia conter alguma solução subordinada, que não satisfaz a condição de contorno, pertencente a $l^{2}\left(\mathbb{Z}_{+}, \mathbb{C}\right)$ ). Se supusermos, além disso, a existência de uma solução subordinada da equação (D.1.2), excluímos o espectro absolutamente contínuo, de modo que $Q_{2} \subset \Sigma_{\mathrm{sc}}^{\prime \prime \prime}$. Logo, a relação (F.2.15), juntamente com a hipótese da existência de uma solução subordinada, representa uma condição suficiente à existência de espectro singular-contínuo. Infelizmente, esta não é uma condição necessária, já que pode ocorrer que $\sum_{n=0}^{\infty}\|T(n ; \lambda)\|^{-2}<\infty$ sem que exista uma solução $l^{2}\left(\mathbb{Z}_{+}, \mathbb{C}\right)$.

Por exemplo (vide p. 359 de [LS1]), se adotarmos $a_{n} \equiv 1$ e $b_{n} \equiv 0$, obtemos $u_{n}=$ $c_{1}+c_{2} n$ ( $c_{1}$ e $c_{2}$ constantes ajustáveis pela condição de contorno) como solução geral da equação de Schrödinger (D.1.2). Em particular, $u_{n}^{D}=1+n$ e $u_{n}^{N}=-n$ (soluções que satisfazem, respectivamente, as condições de contorno $u_{-1}^{D}=0$ e $u_{-1}^{N}=1$ ), nenhuma das quais são $l^{2}\left(\mathbb{Z}_{+}, \mathbb{C}\right)$. No entanto, a matriz de transferência

$$
T(n ; \lambda)\left(\begin{array}{cc}
n+1 & -n \\
n & 1-n
\end{array}\right)
$$

tem como norma $\|T(n ; \lambda)\|=\sqrt{2} n+O(1)$, e portanto $\sum_{n=0}^{\infty}\|T(n ; \lambda)\|^{-2}=\sum_{n=0}^{\infty} O\left(n^{-2}\right)<$ $\infty$.

Apesar da condição $\sum_{n=0}^{\infty}\|T(n ; \lambda)\|^{-2}<\infty$ não ser suficiente para a existência de uma solução $l^{2}\left(\mathbb{Z}_{+}, \mathbb{C}\right)$, esta condição tem uma conseqüência importante. Necessitamos da seguinte

Definição F.2.11 Uma solução u(z) da equação de Schrödinger (D.1.2) é dita fortemente subordinada se para qualquer outra solução linearmente independente $v(z)$ for válido o limite

$$
\lim _{n \rightarrow \infty} \frac{u_{n}^{2}+u_{n+1}^{2}}{v_{n}^{2}+v_{n+1}^{2}}=0
$$

É simples ver que qualquer solução fortemente subordinada é também uma solução subordinada. De fato, definindo $S_{w} \equiv \sum_{n=0}^{N+1}\left(w_{n}^{2}+w_{n+1}^{2}\right)$, observamos da definição (F.2.19) que $u_{n}^{2}+u_{n+1}^{2}=o\left(v_{n}^{2}+v_{n+1}^{2}\right)$, de onde concluímos que $S_{u}=o\left(S_{v}\right)$. Segue de uma desigualdade análoga a (F.2.9) (para funções reais) que

$$
\lim _{N \rightarrow \infty} \frac{\|u(\lambda)\|_{N}}{\|v(\lambda)\|_{N}} \leq 2 \lim _{N \rightarrow \infty} \frac{S_{u}}{S_{v}}=2 \lim _{N \rightarrow \infty} \frac{o\left(S_{v}\right)}{S_{v}}=0
$$

o que demonstra nossa afirmação.

Outro resultado importante é o seguinte 
Teorema F.2.12 (Teorema 8.1 de [LS1]) Sejam $A_{0}, A_{1}, \ldots$ matrizes reais unimodulares $2 \times 2$ e seja $T(n)=A_{n} A_{n-1} \cdots A_{0}$. Suponha que

$$
\sum_{n=0}^{\infty} \frac{\left\|A_{n+1}\right\|^{2}}{\|T(n)\|^{2}}<\infty .
$$

Então, existe um vetor unitário $u \in \mathbb{R}^{2}$ tal que para qualquer outro vetor unitário $v$ linearmente independente segue que

$$
\frac{\|T(n) u\|}{\|T(n) v\|} \rightarrow 0
$$

Observação F.2.13 O Teorema F.2.12 é essencialmente uma abstração do teorema ergódico multiplicativo de Ruelle (vide $[R]$ ).

Demonstração. Designemos por $t_{n}:=\|T(n)\|$ e por $a_{n}:=\left\|A_{n}\right\|$ as normas espectrais de $T(n)$ e $A_{n}$, respectivamente. Uma vez que $T(n)$ é uma matriz real unimodular $2 \times 2$, esta possui $t_{n}^{2}$ e $t_{n}^{-2}$ como autovalores, aos quais se associam os autovetores ortonormais $\mathbf{v}_{n}^{+}$e $\mathbf{v}_{n}^{-}$(isto é, $\left(\mathbf{v}_{n}^{+}, \mathbf{v}_{n}^{-}\right)=0$ ). Fazemos $\mathbf{v}_{\theta}=\left(\begin{array}{c}\cos \theta \\ \sin \theta\end{array}\right)$ e definimos $\theta_{n}$ por

$$
\mathbf{v}_{\theta_{n}}=\mathbf{v}_{n}^{-} \text {. }
$$

Obviamente, $\mathbf{v}_{n}^{+}=\mathbf{v}_{\theta_{n}+\pi / 2}$, e assim temos do Teorema espectral (Teorema B.2.6)

$$
\begin{aligned}
\left\|T(n) \mathbf{v}_{\theta}\right\|^{2} & =\left(\mathbf{v}_{\theta}, T^{*}(n) T(n) \mathbf{v}_{\theta}\right) \\
& =t_{n}^{2}\left|\left(\mathbf{v}_{\theta}, \mathbf{v}_{n}^{+}\right)\right|^{2}+t_{n}^{-2}\left|\left(\mathbf{v}_{\theta}, \mathbf{v}_{n}^{-}\right)\right|^{2} \\
& =t_{n}^{2} \sin ^{2}\left(\theta-\theta_{n}\right)+t_{n}^{-2} \cos ^{2}\left(\theta-\theta_{n}\right) .
\end{aligned}
$$

De (F.2.23), para $n+1$,

$$
t_{n+1}^{2} \sin ^{2}\left(\theta_{n}-\theta_{n+1}\right) \leq\left\|T(n+1) \mathbf{v}_{n}^{-}\right\|^{2} \leq a_{n+1}^{2}\left\|T(n) \mathbf{v}_{n}^{-}\right\|^{2}=a_{n+1}^{2} t_{n}^{-2} .
$$

Uma vez que $A_{n+1}$ é unimodular, $t_{n}=\left\|T(n+1) A_{n+1}^{-1}\right\| \leq t_{n+1} a_{n+1}$, de tal modo que

$$
t_{n}^{2} \sin ^{2}\left(\theta_{n}-\theta_{n+1}\right) \leq a_{n+1}^{4} t_{n}^{-2} .
$$

Como $\sin ^{2}(x) \geq\left(\frac{2 x}{\pi}\right)^{2}$, vemos que

$$
\left|\theta_{n}-\theta_{n+1}\right| \leq \frac{\pi}{2} \frac{a_{n+1}^{2}}{t_{n}^{2}}
$$

Concluímos do resultado acima e da condição (F.2.20) que a seqüência $\left(\theta_{n}\right)_{n \geq 1}$ possui um limite $\theta_{\infty}=\lim _{n \rightarrow \infty} \theta_{n}$. Desse modo, a equação (F.2.23) e a estimativa telescópica

$$
\left|\theta_{n}-\theta_{\infty}\right| \leq \sum_{m=n}^{\infty}\left|\theta_{m}-\theta_{m+1}\right| \leq \frac{\pi}{2} \sum_{m=n}^{\infty} \frac{a_{m+1}^{2}}{t_{m}^{2}}
$$


levam a

$$
\left\|T(n) \mathbf{v}_{\theta_{\infty}}\right\|^{2} \leq t_{n}^{2}\left(\theta_{n}-\theta_{\infty}\right)^{2}+t(n)^{-2}
$$

Por outro lado, como $\theta_{n}-\theta_{\infty} \rightarrow 0$, também segue de (F.2.23) que

$$
\left\|T(n) \mathbf{v}_{\pi / 2+\theta_{\infty}}\right\|^{2} \geq \frac{1}{2} t_{n}^{2}
$$

Obtemos de (F.2.25) e (F.2.26)

$$
\frac{\left\|T(n) \mathbf{v}_{\theta_{\infty}}\right\|^{2}}{\left\|T(n) \mathbf{v}_{\pi / 2+\theta_{\infty}}\right\|^{2}} \leq 2\left(\theta_{n}-\theta_{\infty}\right)^{2}+2 t_{n}^{-4} \rightarrow 0
$$

já que $a_{n+1} \neq 0\left(A_{n}, n \in \mathbb{Z}_{+}\right.$é unimodular, por hipótese) e a desigualdade (F.2.20) implicam que $t_{n} \rightarrow \infty$. A relação (F.2.21) segue destas considerações.

Também de grande importância é o

Teorema F.2.14 (Teorema 8.2 de [LS1]) Sob as hipóteses do Teorema F.2.12, suponha que também seja satisfeita a desigualdade

$$
\sum_{n=0}^{\infty}\|T(n)\|\left(\sum_{m=n}^{\infty} \frac{\|A(m+1)\|^{2}}{\|T(m)\|^{2}}\right)^{2}<\infty
$$

Então, existe um vetor unitário $u_{\infty}$ com

$$
\sum_{n=0}^{\infty}\left\|T(n) u_{\infty}\right\|^{2}<\infty
$$

Demonstração. Temos das desigualdades (F.2.24) e (F.2.25) a validade de

$$
\sum_{n=0}^{\infty}\left\|T(n) u_{\infty}\right\|^{2} \leq \sum_{n=0}^{\infty}\|T(n)\|^{2}\left(\sum_{m=n}^{\infty} \frac{\|A(m+1)\|^{2}}{\|T(m)\|^{2}}\right)^{2}+\sum_{n=0}^{\infty}\|T(n)\|^{-2}<\infty
$$

sob a hipótese (F.2.28) e caso $\sum_{n=0}^{\infty} t(n)^{-2}<\infty$. Esta última desigualdade é, no entanto, decorrente mais uma vez de (F.2.20) e do fato de $A_{n+1}$ ser unimodular para todo $n$.

Podemos finalmente reunir os resultados anteriores no importante

Teorema F.2.15 (Teorema 1.7 de [LS1]) Seja L a matriz de Jacobi (D.1.1) com entradas limitadas (ou seja, $\left|a_{n}\right|,\left|b_{n}\right|<\infty$ ) e seja $T(n ; \lambda)$ a matriz de transferência (F.1.3) associada a um par de soluções linearmente independentes da equação de Schrödinger (D.1.2). Se $\sum_{n=0}^{\infty}\|T(n ; \lambda)\|^{-2}<\infty$, então existe uma solução fortemente subordinada (vide Definição F.2.19), $u_{\infty}$, de (D.1.2) que obedece a estimativa

$$
\left\|T(n ; \lambda) u_{\infty}\right\|^{2} \leq\|T(n ; \lambda)\|^{-2}+\|T(n ; \lambda)\|^{2}\left(\sum_{m=n}^{\infty} \frac{1}{\|T(m ; \lambda)\|^{2}}\right)^{2} .
$$


Em particular, se

$$
\sum_{n=0}^{\infty}\left\{\|T(n ; \lambda)\|^{2}\left(\sum_{m=n}^{\infty}\|T(m ; \lambda)\|^{-2}\right)^{2}\right\}<\infty
$$

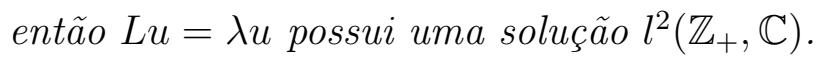

Demonstração. O Teorema F.2.15 é um caso particular dos Teoremas F.2.12 e F.2.14. Devemos, no entanto, efetuar algumas adaptações necessárias. Devemos, por exemplo, realizar as identificações $T(n)=T(n ; \lambda)$ e $A_{n}=T(n, n-1 ; \lambda)$.

Em particular, segue da definição (F.1.2) da matriz de transferência $T(n, n-1 ; \lambda)$ a desigualdade

$$
\|T(n, n-1 ; \lambda)\|^{2} \leq\|T(n, n-1 ; \lambda)\|_{E}^{2} \leq 1+\frac{a_{n-1}^{2}+\left(\lambda-b_{n}\right)^{2}}{a_{n}^{2}}<C<\infty,
$$

onde $\|\cdot\|_{E}$ representa a norma matricial euclideana, e $C$ uma constante, dado que $\left|a_{n}\right|,\left|b_{n}\right|<\infty$ e $a_{n}>0$. A saber, obtemos da hipótese $\sum_{n=0}^{\infty}\|T(n ; \lambda)\|^{-2}<\infty$ a desigualdade

$$
\sum_{n=0}^{\infty} \frac{\|T(n, n-1 ; \lambda)\|^{2}}{\|T(n ; \lambda)\|^{2}} \leq C \sum_{n=0}^{\infty}\|T(n ; \lambda)\|^{-2}<\infty .
$$

Logo, a hipótese do Teorema F.2.12 é satisfeita, e assim existem soluções ortogonais da equação (D.1.2) que obedecem a relação (F.2.21). Provemos que a validade desta condição implica na existência de uma solução fortemente subordinada.

Seja o vetor $\mathbf{u}_{\infty}=\left(u_{k}\right)_{k \geq 0}$, com $u_{k}=\left(T(k ; \lambda) \mathbf{u}_{\theta_{\infty}}\right)_{2}$, a solução da equação de Schrödinger (D.1.2) que satisfaz, em princípio, uma condição de contorno arbitrária. Pela definição (F.1.2) da matriz de transferência, $u_{k}$ é uma solução fortemente subordinada, já que para $v_{k} \equiv\left(T(k ; \lambda) \mathbf{v}_{\theta_{\infty}+\pi / 2}\right)_{2}$, temos de $(\mathrm{F} .2 .27)$

$$
\lim _{k \rightarrow \infty} \frac{u_{k}^{2}+u_{k+1}^{2}}{v_{k}^{2}+v_{k+1}^{2}}=0 .
$$

Mais ainda, as desigualdades (F.2.24), (F.2.25) e a primeira desigualdade de (F.2.31) nos conduzem diretamente à estimativa (F.2.29), concluindo a primeira parte da demonstração.

O fato da solução $\mathbf{u}_{\infty}$ pertencer a $l^{2}\left(\mathbb{Z}_{+}, \mathbb{C}\right)$ é conseqüência direta do Teorema F.2.14, uma vez que a desigualdade (F.2.28) decorre de (F.2.30), dado que $A_{n}=\|T(n, n-1 ; \lambda)\|<$ $C<\infty$. Concluímos assim, a demonstração do teorema.

Caso definamos o conjunto

$$
Q_{3}:=\left\{\lambda \in \mathbb{R}: \sum_{n=0}^{\infty}\left\{\|T(n ; \lambda)\|^{2}\left(\sum_{m=n}^{\infty}\|T(m ; \lambda)\|^{-2}\right)^{2}\right\}<\infty\right\},
$$


segue dos Teoremas E.11 e F.2.15 que $Q_{3} \subset \Sigma_{0} \cup \Sigma_{\text {pp }}^{\prime \prime \prime}$ (a desigualdade $\sum_{n=0}^{\infty}\|T(n ; \lambda)\|^{-2}<$ $\infty$ é automaticamente satisfeita por (F.2.30)). Em particular, excluíndo conjuntos de medidas espectral e de Lebesgue nulas (o que inclui o suporte $\Sigma_{0}$ ), vemos que (F.2.30) fornece uma condição suficiente à existência de espectro (essencial) puramente pontual, a qual, como veremos, é fundamental na caracterização dos operadores esparsos que estudamos (na realidade, usamos uma adaptação do resultado para este caso particular).

Observação F.2.16 O Teorema E.11 nos garante que a solução fortemente subordinada da equação de Schrödinger (D.1.2), resultante da desigualdade (F.2.30), satisfaz a condição de contorno (D.1.9) a menos de um conjunto de medidas de Lebesgue e espectral nulas.

Podemos agrupar os resultados obtidos até então no seguinte

Teorema F.2.17 Sejam $\Sigma_{\mathrm{ac}}^{\prime \prime \prime}, \Sigma_{\mathrm{s}}^{\prime \prime \prime}, \Sigma_{\mathrm{sc}}^{\prime \prime \prime}$ e $\Sigma_{\mathrm{pp}}^{\prime \prime \prime}$ os suportes minimos, respectivamente, das partes absolutamente contínua $\mu_{\mathrm{ac}}$, singular $\mu_{\mathrm{s}}$, singular-contínua $\mu_{\mathrm{sc}}$ e puramente pontual $\mu_{\mathrm{pp}}$ de $\mu$ com respeito a $\kappa$, como definidas no Teorema E.11. Então

(i) $\Sigma_{\text {ac }}^{\prime \prime \prime}=\left\{\lambda: \liminf \inf _{N \rightarrow \infty} \frac{1}{N} \sum_{n=0}^{N}\|T(n ; \lambda)\|^{2}<\infty\right\}$,

(ii) $\Sigma_{\mathrm{s}}^{\prime \prime \prime} \cup \Sigma_{0}=\left\{\lambda \in \mathbb{R}: \lim _{N \rightarrow \infty} \frac{1}{N} \sum_{n=0}^{N}\|T(n ; \lambda)\|^{2}=\infty\right\}$,

(iii) $\Sigma_{\mathrm{sc}}^{\prime \prime \prime} \supset\left\{\lambda \in \mathbb{R}: \sum_{n=0}^{\infty}\|T(n ; \lambda)\|^{-2}=\infty\right\} \cap \Sigma_{0}^{C}$,

(iv) $\Sigma_{\mathrm{pp}}^{\prime \prime \prime} \supset\left\{\lambda \in \mathbb{R}: \sum_{n=0}^{\infty}\left\{\|T(n ; \lambda)\|^{2}\left(\sum_{m=n}^{\infty}\|T(m ; \lambda)\|^{-2}\right)^{2}\right\}<\infty\right\} \cap \Sigma_{0}^{C}$,

em que $\Sigma_{0}:=\{\lambda \in \mathbb{R}$ : existe uma solução subordinada da equação de Schrödinger (D.1.2), porém esta não satisfaz a condição de contorno (D.1.9)\} e $T(n ; \lambda)$ representa a matriz de transferência também associada a (D.1.2).

\section{F.3 Variáveis de Prüfer}

Já que o Teorema F.2.17 relaciona o comportamento assintótico da norma da matriz de transferência aos tipos espectrais, devemos possuir uma ferramenta que nos permita estimar esse comportamento. Esse é justamente o papel desempenhado pelas variáveis de Prüfer no problema que estudamos. Mais especificamente, consideramos o modelo unidimensional definido por (1.4).

Na realidade, trabalhamos com as chamadas variáveis de Prüfer modificadas ou variáveis EFGP, denominadas assim por Kiselev, Last e Simon em [KLS] em homenagem a Eggarter, Pastur, Gredeskul e Figotin, que em uma série de trabalhos (vide Seção 1 de [KLS] para as referências) estenderam as transformações de Prüfer do campo de equações diferenciais ordinárias de segunda ordem ao problema discreto. 
As transformações originalmente propostas por Prüfer se destinavam a solucionar o problema de Sturm-Liouville contínuo, convertendo uma equação de segunda ordem em duas equações de primeira ordem, uma delas dependente apenas de uma variável angular (o "ângulo de Prüfer"); conhecido o ângulo, basta resolver a equação que envolve o "raio de Prüfer" por quadratura. A idéia por trás das variáveis EFGP não é diferente. A saber, definimos a transformação $E F G P^{\dagger}$ como

$$
\mathbf{v}_{\mathbf{k}}:=\left(\begin{array}{c}
R_{k} \cos \theta_{k} \\
R_{k} \sin \theta_{k}
\end{array}\right)=U\left(\begin{array}{c}
u_{k} \\
u_{k-1}
\end{array}\right)=\left(\begin{array}{c}
\sin (\varphi) u_{k-1} \\
u_{k}-\cos (\varphi) u_{k-1}
\end{array}\right)
$$

onde $\left\{u_{k}\right\}_{k \geq 0}$ é uma solução arbitrária da equação de Schrödinger

$$
p_{n} u_{n+1}+p_{n-1} u_{n-1}-\lambda u_{n}=0
$$

$\operatorname{com} \lambda=2 \cos \varphi \in[-2,2], \varphi \in[0, \pi)$ uma parametrização do espectro da matriz de Jacobi $J_{P}$ (definida pela equação (1.13); vide a Seção 2.4 .2 para uma discussão envolvendo o espectro de $\left.J_{P}\right)$, e

$$
U:=\left(\begin{array}{cc}
0 & \sin \varphi \\
1 & -\cos \varphi
\end{array}\right)
$$

Desse modo, se definirmos o vetor $\mathbf{u}_{\mathbf{n}}=\left(\begin{array}{c}u_{n} \\ u_{n-1}\end{array}\right)$, podemos reescrever (F.3.1) como

$$
\mathbf{v}_{\mathbf{k}}=U \mathbf{u}_{\mathbf{k}}
$$

Definimos $R_{n}(\theta)$ impondo que $\theta_{0}=\theta, \theta$ associado à condição de contorno (D.1.4) por (F.3.4), isto é

$$
\mathbf{v}_{0}(\theta)=R_{0}\left(\begin{array}{c}
\cos \theta \\
\sin \theta
\end{array}\right)=U\left(\begin{array}{c}
\cos \alpha \\
\sin \alpha
\end{array}\right)=\left(\begin{array}{c}
\sin \varphi \sin \alpha \\
\cos \alpha-\cos \varphi \sin \alpha
\end{array}\right)
$$

onde $R_{0}^{-2}=1-\cos \varphi \sin 2 \phi$.

Observação F.3.1 Como $\partial \theta / \partial \alpha=-\sin \varphi$, existe, para cada $\varphi \in(0, \pi)$, uma bijeção entre o ângulo de Prüfer inicial $\theta$ e a condição de contorno $\alpha$ em $n=-1$, expressa por (D.1.4).

Necessitamos de alguns resultados antes de prosseguirmos. Seja

$$
\|A\|_{U}:=\left\|U A U^{-1}\right\|
$$

\footnotetext{
${ }^{\dagger}$ Nossa definição difere da definição de Kiselev, Last e Simon, onde as funções $\cos \theta_{k}$ e $\sin \theta_{k}$ são permutadas. Isso não ocasiona mudanças significativas no comportamento das variáveis de Prüfer de uma definição à outra.
} 
a norma matricial associada à matriz inversível $U$ (definida por (F.3.3)), com $\varphi \in[0, \pi)$, onde

$$
\|B\|=\sup _{v \in \mathbb{C}^{2}} \frac{\|B v\|}{\|v\|}
$$

é a norma matricial espectral induzida pela norma vetorial euclideana em $\mathbb{C}^{2}$. Temos a seguinte

Proposição F.3.2 (Marchetti, Wreszinski, Guidi, Angelo) A relação

$$
C^{-1}\|A\| \leq\|A\|_{U} \leq C\|A\|
$$

é válida para

$$
C=\sqrt{\frac{1+|\cos \varphi|}{1-|\cos \varphi|}}
$$

Demonstração. Dado $\mathbf{v}=\left(v_{1}, v_{2}\right) \in \mathbb{C}^{2}$, temos da definição de norma vetorial

$$
\|U \mathbf{v}\|^{2}=\left|v_{1}\right|^{2}+\left|v_{2}\right|^{2}-2 \Re\left(v_{1} \overline{v_{2}}\right) \cos \varphi,
$$

que conjuntamente a

$$
0 \leq\left|v_{1} \pm v_{2}\right|^{2}=\left|v_{1}\right|^{2}+\left|v_{2}\right|^{2}-2 \Re\left(v_{1} \overline{v_{2}}\right)
$$

nos conduz a

$$
(1-|\cos \varphi|)\|\mathbf{v}\|^{2} \leq\|U \mathbf{v}\|^{2} \leq(1+|\cos \varphi|)\|\mathbf{v}\|^{2}
$$

concluímos, portanto, que a norma vetorial $\|\mathbf{v}\|_{U}:=\|U \mathbf{v}\|$ é equivalente a norma $\|\mathbf{v}\|$. Como a matriz $U$ define um isomorfismo em $\mathbb{C}^{2}$,

$$
\|A\|_{U}=\sup _{v \in \mathbb{C}^{2}} \frac{\left\|U A U^{-1} v\right\|}{\|v\|}=\sup _{w \in \mathbb{C}^{2}} \frac{\|U A w\|}{\|U w\|}=\sup _{w \in \mathbb{C}^{2}} \frac{\|A w\|_{U}}{\|w\|_{U}},
$$

e o resultado segue de (F.3.8).

A relação entre a norma da matriz de transferência e o raio de Prüfer é dada pelo

Teorema F.3.3 (Teorema 2.3 de [KLS]) Seja $\delta>0$. Para qualquer par de condições iniciais distintas $\theta_{1}$ e $\theta_{2}$ que satisfazem $0<\left|\theta^{1}-\theta^{2}\right|<\pi / 2$, existem contantes finitas $C_{1}$ $e C_{2}$, não nulas e independentes de $\lambda e\left\{p_{n}\right\}_{n \geq 0}$, tais que

$$
C_{1} \max \left(R_{n}\left(\theta^{1}\right), R_{n}\left(\theta^{2}\right)\right) \leq\|T(n-1 ; \lambda)\| \leq C_{2} \max \left(R_{n}\left(\theta^{1}\right), R_{n}\left(\theta^{2}\right)\right),
$$

segue para $\lambda \in(-2+\delta, 2-\delta)$. 
Observação F.3.4 O Teorema F.3.3 é, na verdade, uma adaptação do Teorema 2.3 de [KLS] ao nosso problema.

Demonstração. É suficiente demonstrarmos o resultado para a norma $\|\cdot\|_{U}$ definida por (F.3.6), já que $\|\cdot\|$ e $\|\cdot\|_{U}$ são equivalentes, como vimos na Proposição F.3.2. Segue imediatamente das definições do vetor $\mathbf{v}$ (equação (F.3.4)), da matriz de transferência $T(n ; \lambda)$ (equação $\left(\right.$ F.1.3)) e da norma $\|\cdot\|_{U}$, a identidade

$$
\left\|T(n-1 ; \lambda) \mathbf{v}_{0}(\theta)\right\|_{U}=R_{n}(\theta),
$$

de onde concluímos, em associação a (F.3.8), que

$$
\|T(n-1 ; \lambda)\| \geq C^{-1} \max _{\theta^{i}}\left\|T(n-1 ; \lambda) \mathbf{v}_{0}\left(\theta^{i}\right)\right\|_{U}=C^{-1} \max \left(R_{n}\left(\theta^{1}\right), R_{n}\left(\theta^{2}\right)\right)
$$

com $C$ definido por (F.3.7), e $\theta^{i} \equiv \theta_{0}^{i}, i=1,2$, os ângulos de Prüfer associados a distintas condições iniciais, como em (F.3.5). Para a segunda desigualdade necessitamos do

Lema F.3.5 (Lema 2.2 de [KLS]) Seja A uma matriz unimodular e defina $\mathbf{u}_{\theta}=(\cos \theta$, $\sin \theta)$. Se $\left|\theta^{1}-\theta^{2}\right| \leq \frac{\pi}{2}$, ent $\tilde{a} O$

$$
\|A\| \leq \sin \left(\frac{1}{2}\left|\theta^{1}-\theta^{2}\right|\right)^{-1} \max \left(\left\|A \mathbf{u}_{\theta^{1}}\right\|,\left\|A \mathbf{u}_{\theta^{2}}\right\|\right) .
$$

Demonstração. Sendo $A$ uma matriz unimodular, existe um ângulo $\theta^{*}$ tal que (vide a demonstração do Teorema F.2.12)

$$
\left\|A \mathbf{u}_{\theta}\right\|^{2}=\|A\|^{2} \sin ^{2}\left(\theta-\theta^{*}\right)+\|A\|^{-2} \cos ^{2}\left(\theta-\theta^{*}\right),
$$

em analogia a (F.2.23), de tal modo que

$$
\|A\|^{2} \leq\left|\sin ^{2}\left(\theta-\theta^{*}\right)\right|^{-1}\left\|A \mathbf{u}_{\theta}\right\|^{2} .
$$

Agora, para qualquer par $\theta^{1}, \theta^{2} \in(0, \pi)$, com $\left|\theta^{1}-\theta^{2}\right|<\pi / 2$,

$$
\left|\sin \left(\theta^{i}-\theta^{*}\right)\right|^{-1} \leq\left|\sin \left(\theta^{1}-\theta^{2}\right) / 2\right|^{-1}
$$

segue para ao menos um $i \in\{1,2\}$. Isso conclui a demonstração do lema.

Como a matriz $U T(n-1 ; \lambda) U^{-1}$ também é unimodular, podemos aplicar o Lema F.3.5 ao nosso problema e obter

$$
\|T(n-1 ; \lambda)\| \leq C \max _{\theta^{i}}\left\|T(n-1 ; \lambda) \mathbf{v}_{0}\left(\theta^{i}\right)\right\|_{U} \leq \widetilde{C} \max \left(R_{n}\left(\theta^{1}\right), R_{n}\left(\theta^{2}\right)\right)
$$

onde $\widetilde{C} \equiv C /\left|\sin \left(\theta^{i}-\theta^{2}\right) / 2\right|$ (usamos o membro direito de (F.3.8) na obtenção da primeira desigualdade acima). Concluímos a demonstração do teorema ao identificar$\operatorname{mos} C_{1}=C^{-1}$ e $C_{2}=\widetilde{C}$. 
O Teorema F.3.3 nos permite uma relação direta entre a norma da matriz de transferência $T(n ; \lambda)$ associada a um par de soluções linearmente independentes da equação de Schrödinger (F.3.2) e seus respectivos raios de Prüfer. A vantagem de se estudar a seqüência $\left\{R_{k}\left(\theta_{0}\right)\right\}_{k \geq 1}$ de raios de Prüfer $^{\dagger}$ reside no fato de sermos capazes de estabelecer, para o modelo com esparsidade, seu comportamento assintótico com precisão; este fato, aliado ao Teorema F.2.17, garante uma caracterização quase completa do espectro, inclusive de sua parte singular-contínua, como veremos. Esse resultado somente é possível sob a hipótese de distribuição uniforme dos ângulos de Prüfer, idéia explorada por Marchetti et. al. em [MWGA]. Estas questões são discutidas em detalhes no Capítulo 3.

${ }^{\dagger} \theta_{0}$ é o ângulo de Prüfer inicial, associado univocamente a uma solução que satisfaz uma condição de contorno, em princípio, arbitrária; vide a Observação F.3.1. 


\section{Apêndice G}

\section{Medidas e dimensão Hausdorff}

O objetivo desse apêndice é apresentar as definições de medida e dimensão Hausdorff, bem como descrever os aspectos mais úteis (nesse trabalho) da teoria da decomposição de medidas de Borel finitas com respeito a esses dois objetos. Uma discussão mais completa se encontra na Seção 4 de [L1].

Começamos com uma série de definições.

Definição G.1 Uma medida de Borel $\mu$, definida em $\mathbb{R}$, é dita $\sigma$-finita se $\mathbb{R}=\bigcup_{j=1}^{\infty} S_{j}$ e $\mu\left(S_{j}\right)<\infty$ para todo $j$. Dizemos, de forma semelhante, que um subconjunto de Borel $S \subseteq \mathbb{R}^{\dagger}$ possui medida $\mu \sigma$-finita se $S=\bigcup_{j=1}^{\infty} S_{j}$ e $\mu\left(S_{j}\right)<\infty$ para cada $j$.

Definição G.2 Seja $S$ um subconjunto de Borel de $\mathbb{R}$. Uma coleção contável de intervalos $\left\{b_{\nu}\right\}_{\nu=1}^{\infty}$ é chamada de $\delta$-cobertura de $S$ se $S \subset \bigcup_{\nu=1}^{\infty} b_{\nu}$ e $\left|b_{\nu}\right|<\delta$ para todo $\nu$.

Definição G.3 Seja $\alpha \in[0,1]$. Para qualquer subconjunto $S \subseteq \mathbb{R}$, definimos a medida de Hausdorff $\alpha$-dimensional de $S$ como

$$
h^{\alpha}(S)=\lim _{\delta \downarrow 0} Q_{\alpha, \delta}(S)
$$

onde

$$
Q_{\alpha, \delta}(S)=\inf \left\{\sum_{\nu=1}^{\infty}\left|b_{\nu}\right|^{\alpha}:\left|b_{\nu}\right|<\delta ; S \subset \bigcup_{\nu=1}^{\infty} b_{\nu}\right\},
$$

o infimo tomado sobre todas as $\delta$-coberturas de $S$.

Esta medida pode ser vista como uma interpolação contínua entre a medida de contagem quando $\alpha=0$ (que associa a cada conjunto $S$ seu número de pontos) e a medida de Lebesgue quando $\alpha=1$. Fica claro a partir das relações (G.2) e (G.1) que $h^{\alpha}(S)$ é uma medida exterior em $\mathbb{R}$, e que sua restrição a conjuntos de Borel é uma medida de Borel (vide $[\mathrm{F}]$ ).

†Os conjuntos de Borel são subconjuntos de $\mathbb{R}$ pertencentes a menor $\sigma$-álgebra gerada pelo conjunto de todos intervalos abertos da reta. 
Observação G.4 Mais precisamente, para $\alpha<1$, $h^{\alpha}$ não é $\sigma$-finita. Além do mais, $h^{\alpha}$ não é regular no sentido usual de ser externamente regular por conjuntos abertos $e$ internamente regular por compactos. $h^{\alpha}$ é, no entanto, $G_{\delta}$ externamente regular e $F_{\sigma}$ internamente regular no seguinte sentido: existe, para qualquer conjunto de Borel $S \subseteq \mathbb{R}$, um conjunto $G_{\delta}, S_{1}$, tal que $S \subseteq S_{1}$ e $h^{\alpha}(S)=h^{\alpha}\left(S_{1}\right)$. Além disso, se $h^{\alpha}(S)<\infty$, então existe um conjunto $F_{\sigma}, S_{2}$, tal que $S_{2} \subseteq S$ e $h^{\alpha}(S)=h^{\alpha}\left(S_{2}\right)$.

Para $\beta<\alpha<\gamma$,

$$
\delta^{\alpha-\gamma} Q_{\gamma, \delta}(S) \leq Q_{\alpha, \delta}(S) \leq \delta^{\alpha-\beta} Q_{\beta, \delta}(S)
$$

segue para quaisquer $\delta>0$ e $S \subset \mathbb{R}$. Desse modo, se $h^{\alpha}(S)<\infty$, então $h^{\gamma}(S)=0$ para $\gamma>\alpha$; se $h^{\alpha}(S)>0$, então $h^{\beta}(S)=\infty$ para $\beta<\alpha$. Assim, para todo conjunto de Borel $S$, existe um único $\alpha_{S}$ tal que $h^{\alpha}(S)=0$ se $\alpha>\alpha_{S}$ e $h^{\alpha}(S)=\infty$ se $\alpha \leq \alpha_{S}$. O número $\alpha_{S}$ é a chamada dimensão Hausdorff do conjunto $S$.

As definições de medida e dimensão Hausdorff nos levam a uma extensa coleção de noções de continuidade e singularidade, expressas pelas seguintes definições:

Definição G.5 Sejam $\mu$ uma medida de Borel em $\mathbb{R}$ e $\alpha \in[0,1]$.

(i) $\mu$ é dita $\alpha$-contínua se $\mu(S)=0$ para todo conjunto $S$ com $h^{\alpha}(S)=0$.

(ii) $\mu$ é dita fortemente $\alpha$-contínua se $\mu(S)=0$ para todo conjunto de Borel $S$ com medida Hausdorff $h^{\alpha} \sigma$-finita.

(iii) $\mu$ é dita $\alpha$-singular se suportada em um conjunto $S$ tal que $h^{\alpha}(S)=0$.

(iv) $\mu$ é dita quase $\alpha$-singular se suportada em um conjunto $S$ com medida Hausdorff $h^{\alpha}$ $\sigma-f i n i t a$.

(v) $\mu$ é dita absolutamente contínua com respeito a $h^{\alpha}$ se $d \mu=f(x) d h^{\alpha}$ para alguma função de Borel $f \in L^{1}\left(\mathbb{R}, d h^{\alpha}\right)$.

Observação G.6 Se $\mu$ é $\sigma$-finita, então segue do Teorema de Radon-Nikodym (Teorema 6.9 de [Ru]) que $\mu$ é absolutamente contínua com respeito a $h^{\alpha}$ se, e somente se, esta for $\alpha$-contínua e quase $\alpha$-singular.

Definição G.7 Sejam $\mu$ uma medida de Borel em $\mathbb{R}$ e $\alpha \in[0,1]$.

(i) $\mu$ é dita $\alpha$-dimensional contínua se $\mu(S)=0$ para todo conjunto $S$ com $\alpha_{S}<\alpha$.

(ii) $\mu$ é dita fortemente $\alpha$-dimensional contínua se $\mu(S)=0$ para todo conjunto $S$ com $\alpha_{S} \leq \alpha$.

(iii) $\mu$ é dita $\alpha$-dimensional singular se suportada em um conjunto $S=\bigcup_{j=1}^{\infty} S_{j}$, onde $\alpha_{S_{j}}<\alpha$ para todo $j$.

(iv) $\mu$ é dita quase $\alpha$-dimensional singular se suportada em um conjunto $S$ com $\alpha_{S} \leq \alpha$. 
(v) Dizemos que $\mu$ possui dimensão exata $\alpha$ se $\mu$ é simultaneamente $\alpha$-dimensional contínua e quase $\alpha$-dimensional singular.

Observação G.8 Também dizemos que uma medida $\mu$ possui dimensão exata $\alpha$ se, para todo $\epsilon>0$, esta for simultaneamente $(\alpha-\epsilon)$-contínua e $(\alpha+\epsilon)$-singular.

Dados $\mu$, uma medida positiva e finita, e $\alpha \in[0,1]$, definimos a derivada superior Hausdorff como o limite

$$
D_{\mu}^{\alpha}(x) \equiv \limsup _{\epsilon \downarrow 0} \frac{\mu((x-\epsilon, x+\epsilon))}{(2 \epsilon)^{\alpha}} .
$$

A definição (G.3) é uma generalização para medidas de Hausdorff da derivada de Radon-Nikodym D.3.5. Repare que o limite $\epsilon \downarrow 0$ não precisa ser definido. Claramente, se $D_{\mu}^{\alpha}\left(x_{0}\right)<\infty$ para algum $x_{0}$ então, para todo $\beta<\alpha$,

$$
D_{\mu}^{\beta}\left(x_{0}\right)=\limsup _{\epsilon \downarrow 0}(2 \epsilon)^{\alpha-\beta} \frac{\mu\left(\left(x_{0}-\epsilon, x_{0}+\epsilon\right)\right)}{(2 \epsilon)^{\alpha}}=\limsup _{\epsilon \downarrow 0}(2 \epsilon)^{\alpha-\beta} D_{\mu}^{\alpha}\left(x_{0}\right)=0 .
$$

De forma semelhante, se $D_{\mu}^{\alpha}\left(x_{0}\right)>0$ para algum $x_{0}$, então $D_{\mu}^{\beta}\left(x_{0}\right)=\infty$ para todo $\beta>\alpha$. Assim, podemos definir para cada $x_{0}$ a dimensão Hausdorff local, $\alpha\left(x_{0}\right)$, expressa como

$$
\alpha_{\mu}\left(x_{0}\right) \equiv \liminf _{\epsilon \downarrow 0} \frac{\ln \mu\left(\left(x_{0}-\epsilon, x_{0}+\epsilon\right)\right)}{\ln (2 \epsilon)} .
$$

Definamos

$$
\begin{aligned}
& T_{0} \equiv T_{0}(\alpha, \mu) \equiv\left\{x: D_{\mu}^{\alpha}(x)=0\right\} \\
& T_{+} \equiv T_{+}(\alpha, \mu) \equiv\left\{x: 0<D_{\mu}^{\alpha}(x)<\infty\right\} \\
& T_{\infty} \equiv T_{\infty}(\alpha, \mu) \equiv\left\{x: D_{\mu}^{\alpha}(x)=\infty\right\}
\end{aligned}
$$

O resultado seguinte se deve a Rogers e Taylor [RT] e foi extraído de Last [L1]:

Teorema G.9 (Rogers e Taylor [RT]) Se $\mu$ é uma medida de Borel finita em $\mathbb{R}$, então $T_{0}, T_{\infty}$ e $T_{+}$são conjuntos de Borel e satisfazem

(i) $h^{\alpha}\left(T_{\infty}\right)=0$;

(ii) $T_{+}$possui medida de Hausdorff, $h^{\alpha}, \sigma$-finita;

(iii) $\mu\left(S \bigcap T_{+}\right)=0$ para todo $S \operatorname{com} h^{\alpha}(S)=0$;

(iv) $\mu\left(S \cap T_{0}\right)=0$ para todo $S$ com medida de Hausdorff, $h^{\alpha}$, $\sigma$-finita.

Uma vez que os conjuntos $T_{0}, T_{+}$e $T_{\infty}$ são disjuntos e $T_{0} \bigcup T_{+} \cup T_{\infty}=\mathbb{R}$, podemos decompor $\mu$ como $d \mu=\chi_{T_{\infty}} d \mu+\chi_{T_{+}} d \mu+\chi_{T_{0}} d \mu$. Portanto, segue da definição G.5 e do Teorema G.9 o seguinte 
Corolário G.10 (Corolário 4.11 de [L1]) Seja $\mu$ uma medida de Borel finita em $\mathbb{R}$. Então, para cada $\alpha \in[0,1]$, $\mu$ possui uma decomposição única em medidas mutuamente singulares; a saber,

$$
d \mu=d \mu_{\alpha \mathrm{s}}+d \mu_{\alpha \mathrm{ac}}+d \mu_{\mathrm{f} \alpha \mathrm{c}},
$$

onde $d \mu_{\alpha \mathrm{s}}$ é $\alpha$-singular, $d \mu_{\alpha \mathrm{ac}}$ é absolutamente contínua com respeito a $h^{\alpha}$ (suportada em um conjunto de medida $h^{\alpha} \sigma$-finita) e $d \mu_{\mathrm{f} \alpha \mathrm{c}}$ é fortemente $\alpha$-contínua.

Demonstração. Vemos do ítem (i) do Teorema G.9 e do ítem (iii) da Definição G.5 que a restrição da medida $\mu$ ao conjunto $T_{\infty}$ é $\alpha$-singular; os ítens (ii) e (iii) do Teorema G.9, juntamente com os ítens (i) e (iv) da Definição G.5 e com a Observação G.6 implicam que a restrição da medida $\mu$ ao conjunto $T_{+}$é absolutamente contínua com respeito a $h^{\alpha}$; por fim, o ítem (iv) do Teorema G.9, juntamente com o ítem (ii) da Definição G.5 implicam que a restrição da medida $\mu$ ao conjunto $T_{0}$ é fortemente $\alpha$-contínua.

Observação G.11 1. Se $\alpha=0$, segue da definição de derivada superior Hausdorff (G.3), do conjunto $T_{\infty}$ e do Corolário $G .10$ que $d \mu_{\alpha \mathrm{s}}=0$, de modo que a decomposição $d \mu=d \mu_{\alpha \mathrm{ac}}+d \mu_{\mathrm{f} \alpha \mathrm{c}}$ coincide com a decomposição de $\mu$ em partes puramente pontual e contínua. Se $\alpha=1$, temos do item (ii) da Definição G.5 e do Corolário G.10 que $d \mu_{\mathrm{f} \alpha \mathrm{c}}=0$, e a decomposição $d \mu=d \mu_{\alpha \mathrm{s}}+d \mu_{\alpha \mathrm{ac}}$ coincide com a decomposição de $\mu$ em partes singular e absolutamente contínua (com respeito à medida de Lebesgue $\kappa$ ).

2. $d \mu_{\alpha \mathrm{c}} \equiv d \mu_{\alpha \mathrm{ac}}+d \mu_{\mathrm{f} \alpha \mathrm{c}}$ é a parte $\alpha$-contínua de $\mu ; d \mu_{\mathrm{q} \alpha \mathrm{s}} \equiv d \mu_{\alpha \mathrm{s}}+d \mu_{\alpha \mathrm{ac}}$ é a parte quase $\alpha$-singular de $\mu$.

3. O Corolário G.10 permite uma extensão, com respeito a medidas Hausdorff, da decomposição padrão de uma medida de Borel em suas partes contínua e singular (vide o Apêndice B). Podemos obter decomposições análogas com respeito a dimensões Hausdorff, através da Definição G.7 (vide Lema 4.1 e Corolário 4.12 de [L1]). No entanto, restringimo-nos nesse trabalho apenas às decomposições que discutimos.

Tratemos da questão da decomposição do espaço de Hilbert $\mathcal{H}$. Dado um $\alpha \in[0,1]$, definimos os subespaços

$$
\begin{aligned}
& \mathcal{H}_{\alpha \mathrm{c}} \equiv\left\{\psi: \mu_{\psi} \text { é } \alpha-\text { contínua }\right\} \\
& \mathcal{H}_{\alpha \mathrm{s}} \equiv\left\{\psi: \mu_{\psi} \text { é } \alpha \text {-singular }\right\}
\end{aligned}
$$

Teorema G.12 (Teorema 5.1 de [L1]) $\mathcal{H}_{\alpha c}$ e $\mathcal{H}_{\alpha \mathrm{s}}$ são subespaços fechados e mutuamente ortogonais, invariantes pela atuação do operador auto-adjunto $T$ (como definido na Seção D), de modo que

$$
\mathcal{H}=\mathcal{H}_{\alpha \mathrm{c}} \oplus \mathcal{H}_{\alpha \mathrm{s}}
$$


Observação G.13 1. Denotamos $\mathcal{H}_{\alpha \mathrm{c}}$ por subespaço $\alpha$-contínuo e $\mathcal{H}_{\alpha \mathrm{s}}$ por subespaço $\alpha$-singular. Os espectros $\sigma_{\alpha \mathrm{c}}$ e $\sigma_{\alpha \mathrm{s}}$ são definidos como os espectros de $T$ restritos aos respectivos subespaços, designados como espectro $\alpha$-contínuo e espectro $\alpha$-singular. Claramente $\sigma=\sigma_{\alpha \mathrm{c}} \cup \sigma_{\alpha \mathrm{s}}$.

2. Para $\alpha=0, \mathcal{H}_{\alpha \mathrm{s}}=0$ e $\mathcal{H}_{\alpha \mathrm{c}}=\mathcal{H}$. Para $\alpha>0, \mathcal{H}_{\alpha \mathrm{c}}$ é um subespaço de $\mathcal{H}_{\mathrm{c}}$ e $\mathcal{H}_{\mathrm{pp}}$ é um subespaço de $\mathcal{H}_{\alpha \mathrm{s}}$ (vide Observação G.11).

Demonstração. Seja $\left\{e_{n}\right\}_{n=0}^{\infty}$ uma base ortonormal de $\mathcal{H}$ (como na Seção D, por exemplo). Defina o conjunto

$$
T_{\infty}^{\mathcal{H}}=\bigcup_{n=0}^{\infty} T_{\infty}\left(\alpha, \mu_{e_{n}}\right)
$$

e $P_{T_{\infty}^{\mathcal{H}}}$ a projetor espectral sobre o conjunto $T_{\infty}^{\mathcal{H}}$ (vide a Definição B.2.2). Fica claro a partir do Corolário G.10 e do ítem 2 da Observação G.11 que $\mathcal{H}_{\alpha \mathrm{s}}=P_{T_{\infty}^{\mathcal{H}}} \mathcal{H}$ e que $\mathcal{H}_{\alpha \text { ac }}=\left(I-P_{T_{\infty}^{\mathcal{H}}}\right) \mathcal{H}(I$ o operador identidade em $\mathcal{H})$, de modo que o teorema resulta de fatos conhecidos com respeito às projeções espectrais (vide a Seção B.2). 



\section{Apêndice $\mathbf{H}$}

\section{Caracterização do espectro singular: teoria de Jitomirskaya e Last}

Apresentamos nesse apêndice os principais resultados do trabalho de Jitomirskaya e Last [JL], cujo objetivo principal é o obtenção de técnicas que permitam distinguir os vários tipos de espectro singular-contínuo. Estas são baseadas na classificação desse tipo espectral com respeito a medidas e dimensões Hausdorff como discutido no Apêndice G. Por exemplo, a continuidade ou singularidade com respeito a uma medida de Hausdorff é completamente determinada, a menos de um conjunto de medida de Lebesgue nula, a partir do conhecimento do comportamento local de escala da medida espectral, associado ao comportamento assintótico das soluções linearmente independentes da equação de Schrödinger (D.1.2).

Para tanto, definimos para todo $\varepsilon>0$, um comprimento $l(\varepsilon) \in(0, \infty)$ através da identidade

$$
\left\|u^{D}(z)\right\|_{l(\varepsilon)}\left\|u^{N}(z)\right\|_{l(\varepsilon)}=\frac{1}{2 \varepsilon}
$$

(vide equação $(1.12)$ de $[\mathrm{JL}]$ ), em que $\|\cdot\|_{l}$ denota a norma $l^{2}\left(\mathbb{Z}_{+}, \mathbb{C}\right)$ dada por (E.2), e $u^{D}(z), u^{N}(z)$, representam as soluções da equação sujeitas às condições iniciais (D.1.9).

Observação H.1 Poderíamos definir o comprimento l em termos de uma relação análoga a (H.1) envolvendo as soluções ortogonais $u^{\alpha}(z)$ e $u^{\alpha_{*}}(z)$, que satisfazem as condições iniciais (D.1.6), associadas à condição de contorno $\alpha \in[0, \pi)$.

Uma vez que no máximo uma das soluções $u^{D}(z), u^{N}(z)$, pertence a $l^{2}\left(\mathbb{Z}_{+}, \mathbb{C}\right)$ (graças à constância do Wronskiano), o membro esquerdo de (H.1) é uma função monótona crescente em $l$, que se anula em $l=0$ e diverge no limite $l \rightarrow \infty$. Por outro lado, o membro direito de (H.1) é uma função monótona decrescente em $\varepsilon$, que diverge quando $\varepsilon \rightarrow 0$. Concluímos, portanto, que $l(\varepsilon)$ é uma função bem definida, contínua e monótona decrescente, que diverge à medida que $\varepsilon \rightarrow 0$.

O resultado central de [JL] é a seguinte desigualdade, que relaciona a função $m_{\infty}$ de Weyl-Titchmarsh (para $z \in \mathbb{C}_{+}$) às soluções $u^{D}(z)$ e $u^{N}(z)$. 
Teorema H.2 (Teorema 1.1 de [JL]) Seja L a matriz de Jacobi (D.1.1) associada ao operador auto-adjunto $T$, que satisfaz a condição de contorno (D.1.9) . Dado $\varepsilon>0$, a desigualdade

$$
\frac{5-\sqrt{24}}{m_{\infty}(\lambda+i \varepsilon)} \leq \frac{\left\|u^{D}(\lambda)\right\|_{l(\varepsilon)}}{\left\|u^{N}(\lambda)\right\|_{l(\varepsilon)}} \leq \frac{5+\sqrt{24}}{m_{\infty}(\lambda+i \varepsilon)}
$$

é satisfeita para todo $\lambda \in \mathbb{R}$.

Observação H.3 O Teorema H.2 nos permite recuperar os resultados originais de Gilbert e Pearson $[G P]$. A demonstração, no entanto é um pouco mais simples, graças em parte à definição de $l(\varepsilon)$, dada por (H.1).

Demonstração. Necessitamos do seguinte

Lema H.4 (Lema 3.1 de $[\mathbf{J L}]$ ) Para todo $n \in \mathbb{Z}_{+}, \chi_{n}(z)$ satisfaz a identidade

$$
\chi_{n}(z)=-u_{n}^{N}(\lambda)+m_{\infty}(z) u_{n}^{D}(\lambda)+i \varepsilon u_{n}^{N}(\lambda) \sum_{k=0}^{n} u_{k}^{D}(\lambda) \chi_{k}(z)-i \varepsilon u_{n}^{D}(\lambda) \sum_{k=0}^{n} u_{k}^{N}(\lambda) \chi_{k}(z)
$$

onde $\chi(z)$ é definida por (D.1.10), e $u^{D}(\lambda)$ e $u^{N}(\lambda)$ são as soluções da equação de Schrödinger (D.1.2) $(z=\lambda)$ que satisfazem as condições iniciais (D.1.5).

Observação H.5 A equação (H.3), bem como a equação (E.5), resultam do método da variação das constantes. Note as semelhanças existentes entre ambas.

Demonstração. Denotemos por $\tilde{v}_{n}(\lambda)$ o membro direito da equação (H.3) para $n \in \mathbb{Z}_{+}$, e seja $\tilde{v}_{0}(\lambda)=-1$. Levando mais uma vez em conta a conservação do Wronskiano, não é difícil verificar que $\tilde{v}(\lambda)$ satisfaz

$$
a_{n} \tilde{v}_{n+1}(\lambda)=-a_{n-1} \tilde{v}_{n-1}(\lambda)+\left(\lambda-b_{n}\right) \tilde{v}_{n}(\lambda)+i \varepsilon \chi_{n}(z)
$$

para todo $n \in \mathbb{Z}_{+}$. Uma vez que

$$
a_{n} \chi_{n+1}(z)=-a_{n-1} \chi_{n-1}(z)+\left(\lambda+i \varepsilon-b_{n}\right) \chi_{n}(z)
$$

e que $\tilde{v}_{-1}(\lambda)=\chi_{-1}(z), \tilde{v}_{0}(\lambda)=\chi_{0}(z)$, pelas condições iniciais (D.1.5) e pelas definições de $\tilde{v}(\lambda)$ e $\chi(z)$, segue por indução que $\tilde{v}_{n}(\lambda)=\chi_{n}(z)$ para todo $n \geq-1$.

Obtemos da equação (H.3), por meio da desigualdade de Cauchy-Schwarz, a estimativa

$$
\|\chi(z)\|_{l} \geq\left\|-u^{N}(\lambda)+m_{\infty} u^{D}(\lambda)\right\|_{l}-2 \varepsilon\left\|u^{D}(\lambda)\right\|_{l}\left\|u^{N}(\lambda)\right\|_{l}\|\chi(z)\|_{l}
$$

válida para todo $l \geq 1$. Se tomarmos $l=l(\varepsilon)$, o que implica em $2 \varepsilon\left\|u^{D}(\lambda)\right\|\left\|_{l}\right\| u^{N}(\lambda) \|_{l}=1$, obtemos

$$
2\|\chi(z)\|_{l(\varepsilon)} \geq\left\|-u^{N}(\lambda)+m_{\infty} u^{D}(\lambda)\right\|_{l(\varepsilon)} .
$$


Quadrando os dois membros de (H.4), obtemos da desigualdade (D.1.20) e da definição de $m_{\infty}$, a desigualdade

$$
\frac{4 \Im m_{\infty}(z)}{\varepsilon}>\left\|u^{N}(\lambda)\right\|_{l(\varepsilon)}^{2}+\left|m_{\infty}\right|^{2}\left\|u^{D}(\lambda)\right\|_{l(\varepsilon)}^{2}-2\left|m_{\infty}\right|\left\|u^{D}(\lambda)\right\|_{l(\varepsilon)}\left\|u^{N}(\lambda)\right\|_{l(\varepsilon)} .
$$

Multiplicando os dois membros da desigualdade acima por $2 \varepsilon$, obtemos de (H.1)

$$
8 \Im m_{\infty}(z)>\frac{\left\|u^{N}(\lambda)\right\|_{l(\varepsilon)}}{\left\|u^{D}(\lambda)\right\|_{l(\varepsilon)}}+\left|m_{\infty}\right|^{2} \frac{\left\|u^{D}(\lambda)\right\|_{l(\varepsilon)}}{\left\|u^{N}(\lambda)\right\|_{l(\varepsilon)}}-2\left|m_{\infty}\right|
$$

o que resulta em

$$
\left|m_{\infty}\right|^{2} \frac{\left\|u^{D}(\lambda)\right\|_{l(\varepsilon)}}{\left\|u^{N}(\lambda)\right\|_{l(\varepsilon)}}-10\left|m_{\infty}\right|+\frac{\left\|u^{N}(\lambda)\right\|_{l(\varepsilon)}}{\left\|u^{D}(\lambda)\right\|_{l(\varepsilon)}}<0,
$$

já que $\Im m_{\infty}(z) \leq\left|m_{\infty}\right|$. Obtemos, resolvendo desigualdade quadrática (H.5) para $\left|m_{\infty}\right|$,

$$
(5-\sqrt{24}) \frac{\left\|u^{N}(\lambda)\right\|_{l(\varepsilon)}}{\left\|u^{D}(\lambda)\right\|_{l(\varepsilon)}} \leq\left|m_{\infty}\right| \leq(5+\sqrt{24}) \frac{\left\|u^{N}(\lambda)\right\|_{l(\varepsilon)}}{\left\|u^{D}(\lambda)\right\|_{l(\varepsilon)}},
$$

de onde concluímos o teorema.

Em particular, segue do Teorema H.2 o

Teorema H.6 (Teorema 1.2 de [JL]) Seja L a matriz de Jacobi associada ao operador auto-adjunto T, que satisfaz a condição de contorno (D.1.9). Se $\mu$ representa sua medida espectral, então, para $\lambda \in \mathbb{R}$ e $\alpha \in[0,1]$,

$$
\limsup _{\varepsilon \rightarrow 0} \frac{\mu((\lambda-\varepsilon, \lambda+\varepsilon))}{(2 \varepsilon)^{\alpha}}=\infty
$$

se, e somente se,

$$
\liminf _{l \rightarrow \infty} \frac{\left\|u^{D}\right\|_{l}}{\left\|u^{N}\right\|_{l}^{b}}=0
$$

onde $b \equiv \alpha /(2-\alpha)$.

Demonstração. Para quaisquer duas funções $f(\varepsilon)$ e $g(\varepsilon)$, escrevemos $f \sim g$ se existirem duas constantes positivas $C_{1}$ e $C_{2}$ tais que $C_{1} f<g<C_{2} f$ para todo $\varepsilon>0$. De (H.1) e de (H.2) obtemos

$$
\varepsilon^{1-\alpha}\left|m_{\infty}(\lambda+i \varepsilon)\right| \sim\left\|u^{N}\right\|_{l(\varepsilon)}^{\alpha-1}\left\|u^{D}\right\|_{l(\varepsilon)}^{\alpha-1} \frac{\left\|u^{N}\right\|_{l(\varepsilon)}}{\left\|u^{D}\right\|_{l(\varepsilon)}}=\left(\frac{\left\|u^{N}\right\|_{l(\varepsilon)}^{b}}{\left\|u^{D}\right\|_{l(\varepsilon)}}\right)^{2-\alpha},
$$

de onde segue que

$$
\limsup _{\varepsilon \downarrow 0} \varepsilon^{1-\alpha}\left|m_{\infty}(\lambda+i \varepsilon)\right|=\infty \quad \Leftrightarrow \quad \liminf _{l \rightarrow \infty} \frac{\left\|u^{D}\right\|_{l(\varepsilon)}}{\left\|u^{N}\right\|_{l(\varepsilon)}^{b}}=0 .
$$

Necessitamos, antes prosseguirmos, do 
Teorema H.7 (Teorema 3.1 de [DJLS]) Fixemos $\mu, x_{0}$ e $\alpha \in[0,1]$. Então $D_{\mu}^{\alpha}\left(x_{0}\right)$, $Q_{\mu}^{(1-\alpha)}\left(x_{0}\right)$ e $R_{\mu}^{(1-\alpha)}\left(x_{0}\right)$ são ou todos infinitos, ou todos nulos, ou todos pertencentes a $(0, \infty)$, onde definimos

$$
\begin{aligned}
& Q_{\mu}^{(1-\alpha)}\left(x_{0}\right)=\lim \sup _{\varepsilon \downarrow 0} \varepsilon^{(1-\alpha)} \Im \int \frac{d \mu(y)}{y-x-i \varepsilon}, \\
& R_{\mu}^{(1-\alpha)}\left(x_{0}\right)=\lim \sup _{\varepsilon \downarrow 0} \varepsilon^{(1-\alpha)}\left|\int \frac{d \mu(y)}{y-x-i \varepsilon}\right| .
\end{aligned}
$$

Demonstração. O Teorema H.7 resulta dos seguintes

Lema H.8 (Lema 3.2 de [DJLS]) Para todo $\gamma \leq 1$, vale

$$
D_{\mu}^{1-\gamma}\left(x_{0}\right) \leq 2 Q_{\mu}^{\gamma}\left(x_{0}\right) \leq 2 R_{\mu}^{\gamma}\left(x_{0}\right)
$$

Demonstração. Defina $M_{\mu}^{\delta}\left(x_{0}\right)=\mu\left(x_{0}-\delta, x_{0}+\delta\right)$. Fica claro que

$$
\Im \int_{\infty}^{\infty} \frac{d \mu(y)}{y-x_{0}-i \varepsilon}=\varepsilon \int_{\infty}^{\infty} \frac{d \mu(y)}{\left(y-x_{0}\right)^{2}+\varepsilon^{2}} \geq \frac{1}{2 \varepsilon} M_{\mu}^{\varepsilon}\left(x_{0}\right)
$$

onde usamos $\frac{\varepsilon}{\left(y-x_{0}\right)^{2}+\varepsilon^{2}} \geq \frac{1}{2 \varepsilon}$ caso $y \in\left[x_{0}-\varepsilon, x_{0}+\varepsilon\right]$. Dessa forma,

$$
\varepsilon^{\gamma} \Im \int_{\infty}^{\infty} \frac{d \mu(y)}{y-x_{0}-i \varepsilon} \geq \frac{1}{2 \varepsilon^{1-\gamma}} M_{\mu}^{\varepsilon}\left(x_{0}\right)
$$

e portanto, segue a primeira desigualdade do lema, com $\gamma=1-\alpha \cdot Q_{\mu}^{\gamma}\left(x_{0}\right) \leq R_{\mu}^{\gamma}\left(x_{0}\right)$ é trivial, já que $\Im z \leq|z|$ segue para qualquer $z \in \mathbb{C}$.

Lema H.9 (Lema 3.3 de [DJLS]) Seja $\alpha<1$. Se $D_{\mu}^{\alpha}\left(x_{0}\right)<\infty$ (ou $\left.D_{\mu}^{\alpha}\left(x_{0}\right)=0\right)$, então $R_{\mu}^{1-\alpha}\left(x_{0}\right) \leq \infty\left(\right.$ ou $\left.R_{\mu}^{1-\alpha}\left(x_{0}\right)=0\right)$.

Demonstração. Suponha primeiramente que $D_{\mu}^{\alpha}\left(x_{0}\right)<\infty$. Caso $\alpha=0$, é simples notar que $D_{\mu}^{0}\left(x_{0}\right)=\lim \sup _{\varepsilon \downarrow 0} \mu\left(x_{0}-\epsilon, x_{0}+\epsilon\right)<\infty($ ou $=0)$ implica diretamente em $R_{\mu}^{1-\alpha}\left(x_{0}\right)=\lim \sup _{\varepsilon \downarrow 0} \varepsilon \int_{-\infty}^{\infty} \frac{d \mu(y)}{\left|y-x_{0}+i \varepsilon\right|}<\infty($ ou $=0)$. Analisemos, portanto, o caso $\alpha>0$. Por hipótese,

$$
M_{\mu}^{\delta}\left(x_{0}\right) \leq C \delta^{\alpha}
$$


e assim, para $\gamma=1-\alpha$,

$$
\begin{aligned}
\limsup _{\varepsilon \downarrow 0} \varepsilon^{\gamma} \int_{-\infty}^{\infty} \frac{d \mu(y)}{\left|y-x_{0}+i \varepsilon\right|} & \leq \limsup _{\varepsilon \downarrow 0} \varepsilon^{\gamma} \int_{-\infty}^{\infty} \frac{d \mu(y)}{\left[\left(x_{0}-y\right)^{2}+\varepsilon^{2}\right]^{1 / 2}} \\
& =\limsup _{\varepsilon \downarrow 0} \varepsilon^{\gamma} \int_{0}^{1} \frac{1}{\left(\delta^{2}+\varepsilon^{2}\right)^{1 / 2}}\left[d_{\delta} M_{\mu}^{\delta}\left(x_{0}\right)\right] \\
& =\limsup _{\varepsilon \downarrow 0} \varepsilon^{\gamma} \int_{0}^{1} \frac{\delta}{\left(\delta^{2}+\varepsilon^{2}\right)^{3 / 2}} M_{\mu}^{\delta}\left(x_{0}\right) d \delta \\
& \leq \lim _{\varepsilon \downarrow 0} C \varepsilon^{\gamma} \int_{0}^{1} \frac{\delta^{\alpha+1}}{\left(\delta^{2}+\varepsilon^{2}\right)^{3 / 2}} d \delta \\
& \leq \lim _{\varepsilon \downarrow 0} C \int_{0}^{\varepsilon^{-1}} \frac{\delta^{\alpha+1}}{\left(\delta^{2}+1\right)^{3 / 2}} d \delta<\infty
\end{aligned}
$$

a primeira igualdade surge se notarmos que

$$
\lim _{\varepsilon \downarrow 0} \varepsilon^{\gamma} \int_{\left|y-x_{0}\right|>1} \frac{d \mu(y)}{\left|y-x_{0}+i \varepsilon\right|}=0,
$$

já que $\gamma>0$; a segunda igualdade se origina de uma integração por partes, pois o termo de fronteira

$$
\lim \sup _{\varepsilon \downarrow 0}\left(\frac{\varepsilon^{\gamma}}{\left(\delta^{2}+1\right)^{1 / 2}} M_{\mu}^{1}\left(x_{0}\right)-\varepsilon^{\alpha} M_{\mu}^{0}\left(x_{0}\right)\right)
$$

se anula, uma vez que $\alpha>0$; a última identidade é fruto da desigualdade $\alpha<1$, pois a integral é finita no limite $\varepsilon^{-1} \rightarrow \infty: \delta^{\alpha+1} /\left(\delta^{2}+1\right)^{3 / 2}=\delta^{\alpha-2} /\left(\delta^{-2}+1\right)^{3 / 2} \in L^{1}\left(\mathbb{R}_{+}^{0}\right)$. Demonstramos, portanto que $D_{\mu}^{\alpha}\left(x_{0}\right)<\infty \Rightarrow R_{\mu}^{1-\alpha}\left(x_{0}\right)<\infty$.

Se $D_{\mu}^{\alpha}\left(x_{0}\right)=0$, então a estimativa (H.9) segue para $\delta<\delta_{0}$, onde $C$ pode ser feito arbitrariamente pequeno (tomando $\delta_{0}$ pequeno). Repetindo os cálculos acima para $\delta_{0} \mathrm{em}$ substituição a 1 no limite de integração superior, obtemos

$$
R_{\mu}^{1-\alpha}\left(x_{0}\right) \leq C \int_{0}^{\infty} \frac{\delta^{\alpha+1}}{\left(\delta^{2}+1\right)^{3 / 2}} d \delta .
$$

Portanto $D_{\mu}^{\alpha}\left(x_{0}\right)=0 \Rightarrow R_{\mu}^{1-\alpha}\left(x_{0}\right)=0$, já que $C$ é arbitrariamente pequeno, e isso conclui a demonstração do lema.

O Lema H.9, conjuntamente à desigualdade (H.8), nos permite concluir as afirmações do Teorema H.7.

Segue do Teorema H.7 que

$$
\limsup _{\varepsilon \downarrow 0} \varepsilon^{1-\alpha}\left|m_{\infty}(\lambda+i \varepsilon)\right|=\infty \quad \Leftrightarrow \quad D_{\mu}^{\alpha}(\lambda)=\infty
$$


o que conjuntamente à definição (G.3) nos conduz à demonstração do Teorema H.6.

O Teorema H.6 nos permite estabelecer propriedades das medidas espectrais (como singularidade e continuidade com respeito a medidas Hausdorff) através da determinação de propriedades locais de escala das soluções das equações de Schrödinger (D.1.2), fornecendo, portanto, uma ferramenta bastante eficaz na análise de tais propriedades em operadores de Schrödinger gerais (em particular, aquele estudado por Marchetti et. al). A ele se combinam dois fatos básicos. O primeiro diz respeito à existência de autovetores generalizados (isto é, soluções da equação de Schrödinger (D.1.2) que satisfazem a condição de contorno) para quase todo $\lambda$ com respeito à medida $\mu$; em particular, a solução $u^{D}(\lambda)$ deve satisfazer as estimativas

$$
\limsup _{l \rightarrow \infty} \frac{\left\|u^{D}\right\|_{l}}{l^{1 / 2} \ln l}<\infty
$$

e

$$
\liminf _{l \rightarrow \infty} \frac{\left\|u^{D}\right\|_{l}}{l^{1 / 2}}<\infty
$$

A desigualdade (H.10) segue de dois resultados:

Proposição H.10 Seja

$$
\tilde{Q}_{0}:=\left\{\lambda: \liminf _{N \rightarrow \infty} \frac{1}{N} \sum_{n=0}^{N}\left|u_{n}^{D}(\lambda)\right|^{2}<\infty\right\} .
$$

Então $\mu\left(\mathbb{R} \backslash \tilde{Q}_{0}\right)=0$, ou seja, $\lambda \in \tilde{Q}_{0}$ para quase todo $\lambda$ com respeito à medida espectral $\mu$.

Observação H.11 A Proposição H.10 nada mais é que uma adaptação da Proposição F.2.5 para o autovetor $u^{D}(\lambda)$. Repare que agora o resultado não se restringe apenas ao espectro absolutamente contínuo do operador auto-adjunto $T$. Vale destacar que caso tivéssemos optado pela condição de contorno (D.1.4), $u^{\alpha}(\lambda)$ (solução que satisfaz (D.1.4)) substituiria $u^{D}(\lambda)$.

Teorema H.12 (Teorema $3.10[\mathrm{LS1}]$ ) Fixemos $\delta>0$. Para quase todo $\lambda$ com respeito à medida $\mu$, temos, para todo $l \geq 2$,

$$
\frac{1}{l} \sum_{n=0}^{l}\left|u_{n}^{D}(\lambda)\right|^{2} \leq C_{\lambda}(\ln l)^{1+\delta} .
$$

Demonstração. Definamos a função $g_{k}(\lambda)=2^{-k} \sum_{n=0}^{2^{k}}\left|u_{n}(\lambda)\right|^{2}$. Então, segue da Proposição H.10 (vide a demonstração da Proposição F.2.5) que $\int g_{k}(\lambda) d \mu(\lambda)=1$, e 
portanto, que $\sum_{k=0}^{\infty} k^{-1-\delta} g_{k}(\lambda) \in L^{1}(\mathbb{R}, d \mu)$. Isto, em particular, resulta na desigualdade $g_{k}(\lambda) \leq \widetilde{C}_{\lambda} k^{1+\delta}$ para quase todo $\lambda$ com respeito a $\mu$. Seja $2^{k-1} \leq l \leq 2^{k}$. Então

$$
\frac{1}{l} \sum_{n=0}^{l}\left|u_{n}^{D}(\lambda)\right|^{2} \leq \frac{1}{2^{k-1}} \sum_{n=0}^{2^{k}}\left|u_{n}^{D}(\lambda)\right|^{2} \leq 2 \widetilde{C}_{\lambda} k^{1+\delta} \leq C_{\lambda}(\ln l)^{1+\delta}
$$

$C_{\lambda}=2\left(1+\frac{1}{\ln 2}\right) \widetilde{C}_{\lambda}$. Isso completa a demonstração do teorema.

Em particular, para $\delta=1$, a relação (H.12) se transforma em (H.10) (lembre-se que quando o limite de uma seqüência existe, este coincide com seus limites inferior e superior).

Já a desigualdade (H.11) segue diretamente da Proposição H.10.

O segundo fato que usamos (em conjunto com o Teorema H.6) para a caracterização da medida espectral com respeito à medida Hausdorff é a constância do Wronskiano. Explicitamente,

$$
W\left[u^{N}, u^{D}\right](n)=a_{n}\left(u_{n+1}^{N} u_{n}^{D}-u_{n+1}^{N} u_{n}^{D}\right)=1
$$

para todo $n \in \mathbb{Z}_{+}$, o que resulta em

$$
\left\|u^{D}(\lambda)\right\|_{l}\left\|u^{N}(\lambda)\right\|_{l} \geq \frac{1}{2 \sqrt{2}}\left(\sum_{n=0}^{[l]-1}\left|a_{n}\right|^{-1}+(l-[l])\left|a_{[l]}\right|^{-1}\right) .
$$

Como veremos a seguir, a combinação das relações (H.10)-(H.13) com o Teorema H.6 nos conduz a uma série de resultados envolvendo questões de continuidade e singularidade da medida espectral com respeito a medidas de Hausdorff, cujas deduções usam apenas informações parciais sobre o comportamento assintótico dos autovetores $u^{D}(\lambda)$ e $u^{N}(\lambda)$.

Dos corolários resultantes do Teorema H.6 presentes em [JL], alguns são particularmente interessantes. Começamos pelo

Corolário H.13 (Corolário 4.4 de [JL]) Defina, para todo $l>0$

$$
R_{l} \equiv \frac{1}{2 \sqrt{2}}\left(\sum_{n=0}^{[l]-1}\left|a_{n}\right|^{-1}+(l-[l])\left|a_{[l]}\right|^{-1}\right)
$$

Suponha que para algum $\alpha \in[0,1]$ e todo $\lambda$ pertencente a algum conjunto de Borel A, toda solução $v$ da equação de Schrödinger (D.1.2) satisfaça

$$
\limsup _{l \rightarrow \infty} \frac{\|v\|_{l}^{2}}{R_{l}^{2-\alpha}}<\infty
$$

Então, a restrição $\mu(A \cap \cdot)$ é $\alpha$-contínua. 
Observação H.14 O Corolário H.13 nos diz que o conjunto de parâmetros $\lambda$ para os quais somente existem soluções limitadas está associado à parte contínua (com respeito $\grave{a}$ medida Hausdorff $\left.h^{\alpha}, \alpha \in[0,1]\right)$ da medida espectral $\mu$. Isto é conseqüência imediata da teoria de subordinância de Gilbert e Pearson, discutida no Apêndice E (trata-se, na realidade, da generalização do fato das soluções limitadas estarem associadas ao espectro absolutamente contínuo no caso da medida de Lebesgue $(\alpha=1))$.

Demonstração. Seja $\lambda \in A$. Temos de (H.13) a desigualdade $\left\|u^{D}(\lambda)\right\|_{l}\left\|u^{N}(\lambda)\right\|_{l} \geq R_{l}$, e já que, por hipótese, $\left\|u^{N}(\lambda)\right\|_{l}^{2}<C R_{l}^{2-\alpha}$ para alguma constante $C$, obtemos $\left\|u^{D}(\lambda)\right\|_{l}>$ $C^{-1 / 2} R_{l}^{\alpha / 2}$. Portanto

$$
\frac{\left\|u^{D}(\lambda)\right\|_{l}}{\left\|u^{N}(\lambda)\right\|_{l}^{b}}>C^{-(1+b) / 2} R_{l}^{\alpha / 2-b(2-\alpha) / 2}=(C / 2)^{-(1+b) / 2}>0
$$

com $b=\alpha /(2-\alpha)$. Segue do Teorema H.6 que $\mu(A \cap \cdot)$ é $\alpha$-contínua.

O Corolário H.13 pode ser reescrito em termos das matrizes de transferência $T(n ; \lambda)$ expressas por (F.1.3). Essa abordagem é particularmente interessante quando tratamos o modelo estudado por Marchetti et. al..

Corolário H.15 (Corolário 3.7 de $[\mathrm{CMW}]$ ) Suponha que para algum $\alpha \in[0,1]$ e todo $\lambda$ pertencente a algum conjunto de Borel A,

$$
\limsup _{l \rightarrow \infty} \frac{1}{R_{l}^{2-\alpha}} \sum_{n=0}^{l}\|T(n ; \lambda)\|^{2}<\infty
$$

com $\|\cdot\|$ alguma norma matricial. Então, para todo $\varepsilon>0$, a restrição $\mu(A \cap \cdot)$ é $(\alpha-\varepsilon)-$ continua.

Demonstração. O Teorema F.3.3 afirma que para um par de ângulos de Prüfer iniciais $\theta^{1}, \theta^{2} \in[0, \pi), \theta^{1} \neq \theta^{2}$, existem duas constantes positivas $c_{1}, c_{2}$ tais que (F.3.9) é satisfeita para $\lambda \in(-2+\delta, 2-\delta), \delta$ um número positivo qualquer. Escolhendo $\theta^{1}=0$ e $\theta^{2}=\pi / 2$, obtemos de (F.3.10)

$$
R_{n}^{2}\left(\theta^{1(2)}\right)=\left(u_{n}^{D(N)}\right)^{2}+\left(u_{n-1}^{D(N)}\right)^{2}-2 \cos \varphi u_{n}^{D(N)} u_{n-1}^{D(N)} .
$$

Segue de (F.3.8) a desigualdade

$$
c^{-}\left[\left(u_{n}^{D(N)}\right)^{2}+\left(u_{n-1}^{D(N)}\right)^{2}\right] \leq R_{n}^{2}\left(\theta^{1(2)}\right),
$$

onde $c^{-}=1-|\cos \varphi|$, que conjuntamente a (F.3.9) nos permite obter

$$
\sum_{n=0}^{l}\|T(n ; \lambda)\|^{2} \geq c \max \left\{\left\|u^{D}(\lambda)\right\|_{l+1}^{2},\left\|u^{N}(\lambda)\right\|_{l+1}^{2}\right\} .
$$


Vemos, da hipótese (H.15) e da desigualdade (H.16), que a hipótese (H.14) do Corolário H.13 é satisfeita. Concluímos, portanto, a demonstração do corolário.

Outra aplicação geral do Teorema H.6 relaciona a taxa de decaimento dos autovetores da equação de Schrödinger (D.1.2) a propriedades dimensionais:

Corolário H.16 (Corolário 4.5 de $[\mathbf{J L}]$ ) Defina $R_{l}$ como no Corolário H.13. Suponha que a igualdade

$$
\liminf _{l \rightarrow \infty} \frac{\left\|u^{D}(\lambda)\right\|_{l}^{2}}{R_{l}^{\alpha}}=0
$$

seja satisfeita para todo $\lambda$ pertencente a algum conjunto de Borel A. Então a restrição $\mu(A \cap \cdot)$ é $\alpha$-singular.

Observação H.17 O Corolário H.16 fornece, em um certo sentido, a informação complementar ao Corolário H.13. O primeiro nos dá uma condição suficiente para que a medida $\mu$ seja $\alpha$-singular; já o segundo fornece uma condição suficiente para que esta mesma medida seja $\alpha$-contínua. Repare na semelhança existente entre as condições e as demonstrações de ambos.

Demonstração. Sejam $\lambda \in A$ e $b=\alpha /(2-\alpha)$. Segue novamente de (H.13) que $\left\|u^{N}(\lambda)\right\|_{l}^{b} \geq\left(R_{l} /\left\|u^{D}(\lambda)\right\|_{l}\right)^{b}$. Isto implica em

$$
\liminf _{l \rightarrow \infty} \frac{\left\|u^{D}(\lambda)\right\|_{l}}{\left\|u^{N}(\lambda)\right\|_{l}^{b}} \leq \liminf _{l \rightarrow \infty} \frac{\left\|u^{D}(\lambda)\right\|_{l}^{1+b}}{R_{l}^{b}}=\liminf _{l \rightarrow \infty}\left(\frac{\left\|u^{D}(\lambda)\right\|_{l}^{2}}{R_{l}^{\alpha}}\right)^{1 /(2-\alpha)}=0 .
$$

Segue do Teorema H.6 que $\mu(A \cap \cdot)$ é $\alpha$-singular.

O resultado acima nos diz que se a solução que satisfaz a condição de contorno de Dirichlet (equação (D.1.9)) crescer com qualquer potência menor que $\alpha$, a restrição $\mu(A \cap \cdot)$ é $\alpha$-singular. 



\section{Referências Bibliográficas}

[A] Anderson, P., "Absence of diffusion in certain random lattices", Phys. Rev. 109, 1492-1505 (1958).

[AALR] Abrahams, E. Anderson, P., Liciardello, D., Ramakrishnan, T., "Scaling Theory of Localization: Absence of Quantum Diffusion in Two Dimensions", Phys. Rev. Lett. 42, 673-676 (1979).

[Ahl] Ahlfors, L. V. "Complex Analysis", McGraw Hill (Singapoure), terceira edição (1979).

[Ar] Aronszajn, N., "On a problem of Weyl in the theory of singular Sturm-Liouville equations", Am. J. Math. 79, 597-610 (1957).

[Bre] Breuer, J., "Singular continuous spectrum for the laplacian on certain sparse trees", Commun. Math. Phys. 269, 851-857 (2007).

[Bre1] Breuer, J., "Singular continuous and dense point spectrum for sparse trees with finite dimensions", Probability and Mathematical Physics (eds. D. Dawson, V. Jaksic and B. Vainberg), CRM Proc. and Lecture Notes, 42, 65-83 (2007).

[CMW] Carvalho, S. L., Marchetti, D. H. U., Wreszinski, W. F., "Sparse Block-Jacobi Matrices with Accurate Hausdorff Dimension", J. Math. Anal. Appl., 368, 218234 (2010).

[CMW1] Carvalho, S. L., Marchetti, D. H. U., Wreszinski, W. F., "On the uniform distribution of the Prüfer angles of Jacobi matrices with randomly sparse perturbations ", ArXiv:1006.2849v1.

[C] Chung, K. L., "A Course in Probability Theory", Academic Press (San Diego), terceira edição (2001).

[CL] Coddington, E. A., Levinson, N., "Theory of Ordinary Differential Equations", Krieger Publishing Company (Malabar), edição reimpressa (1984).

[DEL] Davenport, H., Erdös, P., LeVeque, W. J., "On the Weyl's criterion for uniform distribution", Michigan Math. J. 10, 311-314 (1963). 
[D] Deift, P., "Orthogonal Polynomials and Random Matrices: A Riemann-Hilbert Approach", Courant Lecture Notes, American Mathematical Society (New York), primeira edição (2000).

[DK] Deift, P., Killip, R., "On the absolutely continuous spectrum of one-dimensional Schrodinger operators with square summable potentials", Commun. Math. Phys. 203, 341-347 (1999).

[DS] Dinaburg, E. I., Sinai, Ya. G., "The one-dimensional Schrödinger equation with a quasi-periodic potential", Funct. Anal. Appl. 9, 279-289 (1975).

[Do] Donoghue, W., "On the perturbation of the spectra", Comm. Pure Appl. Math. 18, 559-579 (1965).

[Do1] Donoghue, W., "Monotone Matrix Functions and Analytic Continuation (Grundlehren Der Mathematischen Wissenschaften Series, Vol 207)", Springer (1974).

[DJLS] Del Rio, R., Jitomirskaya, S., Last, Y., Simon, B., "Operators with singular continuous spectrum, IV. Hausdorff dimension, rank one perturbations and localization", J. Anal. Math. 69, 153-200 (1996).

[F] Falconer, K. J., "Fractal Geometry", Wiley (Chichester) (1990).

[FHS1] Froese, R., Hasler, D., Spitzer, W., "Transfer matrices, hyperbolic geometry and absolutely continuous spectrum for some discrete Schrödinger operators on graphs", Journ. Funct. Anal. 230, 184-221 (2006).

[FHS2] Froese, R., Hasler, D., Spitzer, W., "Absolutely continuous spectrum for the Anderson model on a tree: a geometric proof of Klein's theorem", Comm. in Math. Phys. 269, 239-257 (2007).

[FMSS] Fröhlich, J., Martinelli, F., Scoppola, E., Spencer, T., "Constructive proof of localization in the Anderson tight binding model "Commun. Math. Phys. 101, 21-46 (1985).

[FS] Fröhlich, J., Spencer, T., "Absence of diffusion in the Anderson tight binding model for large disorder or low energy" Commun. Math. Phys. 88, 151-184 (1983).

[GT] F. Gesztesy and E. Tsekanovskii, "On matrix-valued Herglotz functions", Math. Nachr. 218, 61-138 (2000).

[GP] Gilbert, D. J., Pearson, D. B., "On subordinacy and analysis of the spectrum of one-dimensional Schrödinger operators", J. Math. Anal. Appl. 128, 30-56 (1987).

[GMP] Gold'sheid, S., Molchanov, S., Pasteur, L., Funct. Anal. Appl. 11, 1 (1977). 
[G] Gordon, A. Ya., "Deterministic potencial with a pure point spectrum", Math. Notes 48, 1197-1203 (1990).

[H] Hartman, P., "Ordinary Differential Equations", John Wiley \& Sons (New York), primeira edição (1964).

[HW] Hartman, P., Wintner, A., "Oscillatory and non-oscillatory linear differential equations", Amer. J. Math. 71, 627-649 (1949).

[HJ] Horn, R. A., Johnson, C. R., "Matrix Analysis", Cambridge University Press (Cambridge), primeira edição (1985).

[JL] Jitomirskaya, S., Last, L., "Power-law subordinacy and singular spectra I. Halfline operators", Acta. Math. 183, 171-189 (1999).

[Ka] Kato, T., "Perturbation Theory for Linear Operators", Springer-Verlag (New York), segunda edição (1976).

[KMP] Kirsch, W., Molchanov, S., Pastur, L., "The one-dimensional Schrödinger operator with unbounded potential: The pure point spectrum", Funct. Anal. Appl. 24, 176-186 (1990).

[KLS] Kiselev, A., Last, Y., Simon, B. "Modified Prüfer and EFGP transforms and the spectral analysis of one-dimensional Schrödinger operators", Commun. Math. Phys. 194, 1-45 (1998)

[K] Klein, A., "Extended states in the Anderson model on the Bethe lattice" Adv. Math. 133, 163-184 (1998).

[KP] Khan, S., Pearson, D.B., "Sudordinacy and spectral theory for infinite matrices" Hel. Phys. Acta 65, 505-527 (1992).

[Kre] Kreyszig, E., "Introductory Functional Analysis with Applications", John Wiley \& Sons (1989).

[KR] Krutikov, D., Remling, C., "Schrödinger Operators with Sparse Potentials: Asymptotics of the Fourier Transform of the Spectral Measure", Commun. Math. Phys. 223, 509-532 (2001).

[KN] Kuipers, L., Niederreiter, H., "Uniform Distributions of Sequences ", John Wiley \& Sons (New York), (1974).

[KS1] Kunz, H., Souillard, B. "Sur le spectre des opérateurs aux différences finies aléatoires " Commun. Math. Phys. 78, 201-246 (1980).

[KS2] Kunz, H., Souillard, B. "On the upper critical dimension and the critical exponents of the localization transition" J. Physique Lett. 44, L411 (1983). 
[L] Lancaster, P., Tismenetsky, M., "The Theory of Matrices", Academic Press (San Diego), segunda edição (1985).

[Lan] Landauer, R., "Electrical resistance of disordered one-dimensional lattices" Phil. Mag. 21, 863-867 (1970).

[L1] Last, Y., "Quantum dynamics and decomposition of singular continuous spectra" J. Funct. Anal. 142, 406-445 (1996).

[L2] Last, Y., "Sturm-Liouville operator on infinite intervals", W. O. Amrein, A. M. Hinz, D. B. Pearson (Eds.), Sturm-Liouville Theory: Past and Present, Birkhäuser Verlag (Basel), 99-120 (2005).

[LS1] Last, Y. Simon, B., "Eigenfunctions, transfer matrices, and absolutely continuous spectrum of one-dimensional Schrödinger operators", Invent. Math. 135, 329-367 (1999).

[LS2] Last, Y. Simon, B., "Modified Prüfer and deterministic models with dense point spectrum", J. Funct. Anal. 1544, 513-530 (1998).

[MWGA] Marchetti, D. H. U., Wreszinski, W. F., Guidi, L. F., Angelo, R. M., "Spectral transition in a sparse model and a class of nonlinear dynamical systems" Nonlinearity 20, 765-787 (2007).

[MS] Martinelli, F., Scoppola, E., "Remark on the absence of absolutely continuous spectrum ford-d imensional Schrödinger operators with random potential for large disorder or low energy" Commun. Math. Phys. 97, 465-471 (1985).

[M] Mockenhaupt, G., "Salem sets and restriction properties of Fourier Transforms" Geom. funct. anal. 10, 1579-1587 (2000).

[MT] Mott, N.F., Twose, W. D., Adv. Phys. 10, 107 (1960).

[OW] Opperman, R., Wegner, F., "Disordered Systems with n Orbitals per Site: 1/n expansion", Z.Phys. B34, 327 (1979).

[P] Pearson, D. B., "Singular Continuous Measures in the Scattering Theory", Commun. Math. Phys. 60, 13-36 (1978).

[P1] Pearson, D. B., "Spectral theory - 50 years of progress and a conclusion", Math. Meth. Appl. Sci. 24, 391-403 (2001).

[PBM] Prudnikov, A. P., Brychkov, Yu, A., Marichev, O. I., "Integrals ans Series (Elementary functions) Vol 1 ", Gordon and Breach (London), primeira edição (1986).

[RS1] Reed, M. Simon, B. "Methods of Modern Mathematical Physics I: Functional Analysis", Academic Press (New York), primeira edição (1978). 
[RS2] Reed, M. Simon, B. "Methods of Modern Mathematical Physics II: Fourier Analysis, Self-Adjointness", Academic Press (New York), primeira edição (1978).

[RS3] Reed, M. Simon, B. "Methods of Modern Mathematical Physics III: Scattering Theory", Academic Press (New York), primeira edição (1978).

[RS4] Reed, M. Simon, B. "Methods of Modern Mathematical Physics IV: Analysis of Operators", Academic Press (New York), primeira edição (1978).

[RT] Rogers C. A., Taylor, S. J., "The analysis of additive set functions in Euclidean space", Acta. Math. 101, 273-302 (1959).

[Ru] Rudin, W.., "Real and complex analysis ", McGraw Hill (New York), terceira edição (1987).

[R] Ruelle, D., "Ergodic theory of differentiable dynamical systems", Publ. Math. IHES 50, 276-306 (1979).

[S] Saks, S., "Theory of the integral", Hafner (New York), segunda edição (1937).

[Si] Simon, B., "Spectral Analysis of Rank One Perturbations and Applications", J. Feldman, R. Froese, L. Rosen (Eds.), CRM Proc. and Lecture Notes, Vol 8, Amer. Math. Soc., Providence, RI, 109-149 (1995).

[Si1] Simon, B., "Operators with singular continuous spectrum, VII. Examples with borderline time decay", Commun. Math. Phys. 176, 713-722 (1996).

[SS] Simon, B., Stolz, G., "Operators with singular continuous spectrum V. Sparse potentials", Proc.Amer. Math. Soc. 124, 2073-2080 (1996).

[SiSp] Simon, B., Spencer, T. C.., "Trace class perturbations and the absence of absolutely continuous spectra", Commun. Math. Phys. 125, 113-125 (1989).

[SW] Simon, B., Wolff, T., "Singular continuous spectrum under rank one perturbations and localization for random hamiltonians", Commun. Pure Appl. Math. 39, 75-90 (1986).

[Sp] Spencer, T.C., "The Schrödinger equation with a random potencial - A mathematical review", K. Osterwalder, R. Stora (Eds.), Les Houches 1984, Session XLIII, Part II, North-Holland (Amsterdam), 895-941 (1986).

[Str] Strichartz, R., S., "Fourier asymptotics of fractal measures", J. Funct. Anal. 89, 154-187 (1990).

[T] Teschl, G., "Jacobi Operators and Completely Integrable Nonlinear Lattices", AMS (New York), (2000). 
[TKNN] Thouless, D. J., Kohmoto, M., Nightingale, M. P., den Nijs, M., "Quantized Hall conductance in two-dimensional periodic potencial", Phys. Rev. Lett. 49, 405-408 (1982).

[W] Wilcox, C. H., "Scattering states and wave operators in the abstract theory of scattering", J. Funct. Anal. 12, 257-274 (1973).

[vDK] von Dreifus, H., Klein, A., "A new proof of localization in the Anderson tight binding model", Commun. Math. Phys. 124, 285-299 (1989).

[Z] Zlatloš, A., "Sparse potentials with fractional Hausdorff dimension", J. Funct. Anal. 207, 216-252 (2004). 